\title{
Thermodynamic stability of hexagonal and rhombohedral boron nitride under chemical vapor deposition conditions from van der Waals corrected first principles calculations
}

Henrik Pedersen, Björn Alling, Hans Högberg and Annop Ektarawong

The self-archived postprint version of this journal article is available at Linköping University Institutional Repository (DiVA):

http://urn.kb.se/resolve?urn=urn:nbn:se:liu:diva-160062

N.B.: When citing this work, cite the original publication.

Pedersen, H., Alling, B., Högberg, H., Ektarawong, A., (2019), Thermodynamic stability of hexagonal and rhombohedral boron nitride under chemical vapor deposition conditions from van der Waals corrected first principles calculations, Journal of Vacuum Science \& Technology. A. Vacuum,

Surfaces, and Films, 37(4), 040603. https://doi.org/10.1116/1.5107455

Original publication available at:

https://doi.org/10.1116/1.5107455

Copyright: AIP Publishing

http://www.aip.org/ 


\section{Thermodynamic stability of hexagonal and rhombohedral BN at CVD conditions from van der Waals corrected first principles calculations}

Running title: Stability of h-BN and r-BN at CVD conditions

Running Authors: Pedersen et al.

Henrik Pedersena), Björn Alling, Hans Högberg and Annop Ektarawong ${ }^{\text {b) }}$

Department of Physics, Chemistry and Biology, Linköping University, SE-58183 Linköping, Sweden

a) Electronic mail: henrik.pedersen@liu.se

b) Present addresses:

1) Extreme Condition Physics Research Laboratory, Physics of Energy Materials Research Unit, Department of Physics, Faculty of Science, Chulalongkorn University, Bangkok, 10330, Thailand

2) Thailand Center of Excellence in Physics, Commission on Higher Education, 328 Si Ayutthaya Road, Bangkok, 10400, Thailand

Thin films of boron nitride (BN), particularly the $\mathrm{sp}^{2}$-hybridized polytypes hexagonal $\mathrm{BN}$ (h-BN) and rhombohedral $\mathrm{BN}(\mathrm{r}-\mathrm{BN})$ are interesting for several electronic applications given band gaps in the UV. They are typically deposited close to thermal equilibrium by chemical vapor deposition (CVD) at temperatures and pressures in the regions 1400-1800 $\mathrm{K}$ and 1000-10000 Pa, respectively. In this letter, we use van der Waals corrected density functional theory and thermodynamic stability calculations to determine the stability of r$\mathrm{BN}$ and compare it to that of $\mathrm{h}-\mathrm{BN}$ as well as to cubic $\mathrm{BN}$ and wurtzitic $\mathrm{BN}$. We find that $\mathrm{r}-\mathrm{BN}$ is the stable $\mathrm{sp}^{2}$-hybridized phase at CVD conditions, while h-BN is metastable. Thus, our calculations suggest that thin films of h-BN must be deposited far from thermal equilibrium. 


\section{INTRODUCTION}

Cubic BN (c-BN) with $\mathrm{sp}^{3}$-hybridization and diamond structure is the most wellknown among BN phases. In 1957, Wentorf synthesized c-BN from a mixture of boron and nitrogen at high temperature and pressures. ${ }^{1}$ The discovery of "Borazon" catalyzed work on the other polytypes of $\mathrm{BN}$, foremost $\mathrm{sp}^{2}$-hybridized hexagonal $\mathrm{BN}(\mathrm{h}-\mathrm{BN})$ "white graphite" and to some extent the less studied wurtzite form (w-BN) that is similar to "hexagonal diamond". In addition to h-BN a rhombohedral $\mathrm{sp}^{2}$-hybridized BN phase (r-BN) was reported 1958 by Hérold. ${ }^{2}$ The two $\mathrm{sp}^{2}$-hybridized BN polytypes differ in their stacking sequence of the basal planes along the c-axis; h-BN exhibits an ABAB... stacking while $\mathrm{r}-\mathrm{BN}$ has an $\mathrm{ABCABC}$... stacking sequence. ${ }^{3}$ Furthermore, the basal planes in h-BN are rotated $180^{\circ}$, with respect to the previous basal plane, around the caxis ([0001]), while in r-BN, each next basal plane is instead shifted along the [1] 00$]$ direction by $1.45 \AA$. The very close structural similarity between h-BN and r-BN is demonstrated in identical in-plane lattice parameters, $2.504 \AA$, and spacing between the basal planes, $3.333 \AA^{4}$

As a thin film, $\mathrm{BN}$ is a promising material for many electronic applications, ${ }^{5-8}$ where the polytype, h-BN or r-BN, are likely to affect the electronic properties: $\mathrm{h}-\mathrm{BN}$ is reported to have a slightly larger band gap than $\mathrm{r}-\mathrm{BN} ; 5.955^{9}$ and $5.7^{10} \mathrm{eV}$, respectively. Typically, $\mathrm{sp}^{2}$-BN films have been deposited by thermally activated Chemical Vapor Deposition (CVD), i.e. close to thermal equilibrium conditions. Epitaxial growth of $\mathrm{sp}^{2}$ $\mathrm{BN}$ thin films by CVD is typically carried out from triethylboron, $\mathrm{B}\left(\mathrm{C}_{2} \mathrm{H}_{5}\right)_{3}(\mathrm{TEB})$, and ammonia, $\mathrm{NH}_{3}$ at low pressures of $1000-10000 \mathrm{~Pa}$ and temperatures in the range of $1100-1500{ }^{\circ} \mathrm{C}(1400-1800 \mathrm{~K}) .{ }^{11}$ The high temperatures needed limit the number of available substrate materials, thus, growth of epitaxial films has been restricted to $\alpha$ $\mathrm{Al}_{2} \mathrm{O}_{3}(0001)$ and $4 \mathrm{H} / 6 \mathrm{H}-\mathrm{SiC}(0001)$ substrates, yielding films oriented around the caxis. ${ }^{12-15} \mathrm{sp}^{2}-\mathrm{BN}$ as a two-dimensional material will consist of a single basal plane with hexagonal symmetry and it is therefore, correctly, refer to as h-BN. However, the authors have previously pointed out the difficulty in structural determination of $\mathrm{sp}^{2}-\mathrm{BN}$ films grown along the c-axis, i.e. the [0001] direction, of thicknesses exceeding a few 
nanometers, and advised to use a more extensive characterization approach to fully understand if a film of $\mathrm{sp}^{2}-\mathrm{BN}$ is $\mathrm{h}-\mathrm{BN}$ or $\mathrm{r}-\mathrm{BN} .^{3}$

In this letter, the authors use van der Waals corrected density functional theory and thermodynamic stability calculations to study the stability of the $\mathrm{sp}^{2}$-BN polytypes at temperatures and pressures relevant to CVD. From the results it is found that at typical CVD temperatures and pressures, $r-\mathrm{BN}$ is the phase predicted to be most stable by thermodynamics.

\section{COMPUTATIONAL DETAILS}

\section{A. First-principles calculations}

The first principles total energies $\mathrm{E}_{0}$ at $\mathrm{T}=0 \mathrm{~K}$ as a function of volume $\mathrm{V}$ of the four BN polytypes are derived from the density functional theory (DFT), ${ }^{16,17}$ in which the project augmented wave (PAW) method ${ }^{18}$, as implemented in the Vienna ab initio simulation package (VASP), ${ }^{19}, 20$ is used and the generalized gradient approximation (GGA), as proposed by Perdew, Burke, and Ernzerhof ${ }^{21}$, is employed for modeling the exchange-correlation interactions. For the four BN polytypes, the energy cutoff for plane waves, included in the expansion of wave functions, is set to $600 \mathrm{eV}$, and the MonkhorstPack k-point mesh ${ }^{22}$ of $15 \times 15 \times 15$ is chosen for the Brillouin zone integration. Since the standard DFT calculations cannot accurately describe the van der Waals interactions, the DFT-D3 correction method with Becke-Jonson damping ${ }^{23,24}$ is used to account for the interactions. For each $B N$ polytype, the optimal equilibrium volume $V_{0}$ at $T=0 \mathrm{~K}$, minimizing $\mathrm{E}_{0}$, is determined by the minimum point of the total energy curve $\mathrm{E}_{0}(\mathrm{~V})$, calculated for a set of different fixed V. During the calculations of $\mathrm{E}_{0}$ at each fixed V, the unit-cell shape and all internal atomic coordinates within the cell are fully relaxed so that the total force, acting on each atom, is less than $10^{-6} \mathrm{eV} / \AA$. Note that, for this particular case, the calculated $\mathrm{E}_{0}(\mathrm{~V})$ are all assured to be converged within an accuracy of 1 $\mathrm{meV} / \mathrm{atom}$ with respect to both the energy cutoff and the number of k-points.

\section{B. Thermodynamic stability calculations}


The relative thermodynamic stability of the four BN polytypes at different fixed pressures $p$ and temperature $T$ is evaluated by minimization of the Gibbs free energy $G$ $(T, p)$;

$$
G(T, p)=E_{0}(V)+F_{v i b}(T, V)+p V_{\text {i }}
$$

where $E_{0}(V)$ is the total energy at $T=0$ directly obtained from the DFT calculations, as described in section II A. $F_{v i b}(T, V)$ is the Helmholtz free energy contributed by the lattice vibrations (phonons), given by;

$$
F_{v i b}(T, V)=\frac{1}{2} \sum_{\mathbf{q},} \hbar \omega(\mathbf{q}, v, V)+k_{B} T \sum_{\mathbf{q},} \ln \left(1-e^{-\hbar\left(\mathbf{q}_{1}, V\right) / k_{B} T}\right) \cdot(2)
$$

$\omega(\mathrm{q}, v, \mathrm{~V})$ is the phonon frequency at the wave vector $\mathrm{q}$ and the band index $v . \hbar$ and $\mathrm{k}_{\mathrm{B}}$ are the reduced Planck constant and the Boltzmann constant, respectively. It is worth noting that, in addition to $\mathrm{q}$ and $v$, the phonon frequencies $\omega$ in Eq. 2 are volume dependent, as the phonon calculations are performed at the quasi-harmonic level using the PHONOPY package for phonon calculations ${ }^{25,26}$, in which the Parlinski-Li-Kawazoe method $^{27}$ with a finite displacement of $0.01 \AA$ is used to calculate the force constants within sufficiently large supercells, i.e., $4 \times 4 \times 1$ primitive hexagonal unit cells for h-BN (64 atoms), $4 \times 4 \times 1$ conventional hexagonal unit cells for $\mathrm{r}-\mathrm{BN}$ (96 atoms), $2 \times 2 \times 2$ conventional cubic unit cells for c-BN (64 atoms), and $3 \times 3 \times 3$ primitive hexagonal unit cells for w-BN (108 atoms). The supercells are then sampled with the $21 \times 21 \times 21$ Monkhorst-Pack q-point grids in order to secure the convergence of the phonon frequencies and thus $F_{v i b}(T, V)$. To determine the term $p V$ and include it in $G(T, p)$ at fixed temperatures, the sums of the first two terms on the right-hand side of Eq. 1 at different fixed volumes are fitted to the third-order Birch-Murnaghan equation of state $(\mathrm{EOS})^{28,29}$. The pressure $\mathrm{p}$ is thus calculated by; 


$$
p=-\left[\frac{\partial\left[E_{0}(V)+F_{v i b}(T, V)\right]}{\partial V}\right]_{T} .
$$

\section{RESULTS}

From minimization of the calculated Gibbs free energy $G(T, p)$ and the methodology described in section II, p-T phase diagrams were constructed to determine the relative thermodynamic stability of the $\mathrm{BN}$ polytypes, h-BN, r-BN, c-BN and w-BN. Fig. 1(a) shows the relative thermodynamic stability between h-BN and r-BN, excluding formation of c-BN and $\mathrm{w}-\mathrm{BN}$. As can be seen $\mathrm{r}-\mathrm{BN}$ is stable at $\mathrm{p}-\mathrm{T}$ conditions typical for CVD, i.e. below atmospheric pressure $0.1 \mathrm{GPa}(1013 \mathrm{hPa})$ and temperatures below 1900 $\mathrm{K}$. h-BN is stable at much higher pressures starting at about $12 \mathrm{GPa}$ and where the h-BN to r-BN transition temperature decreases as the pressure increases.
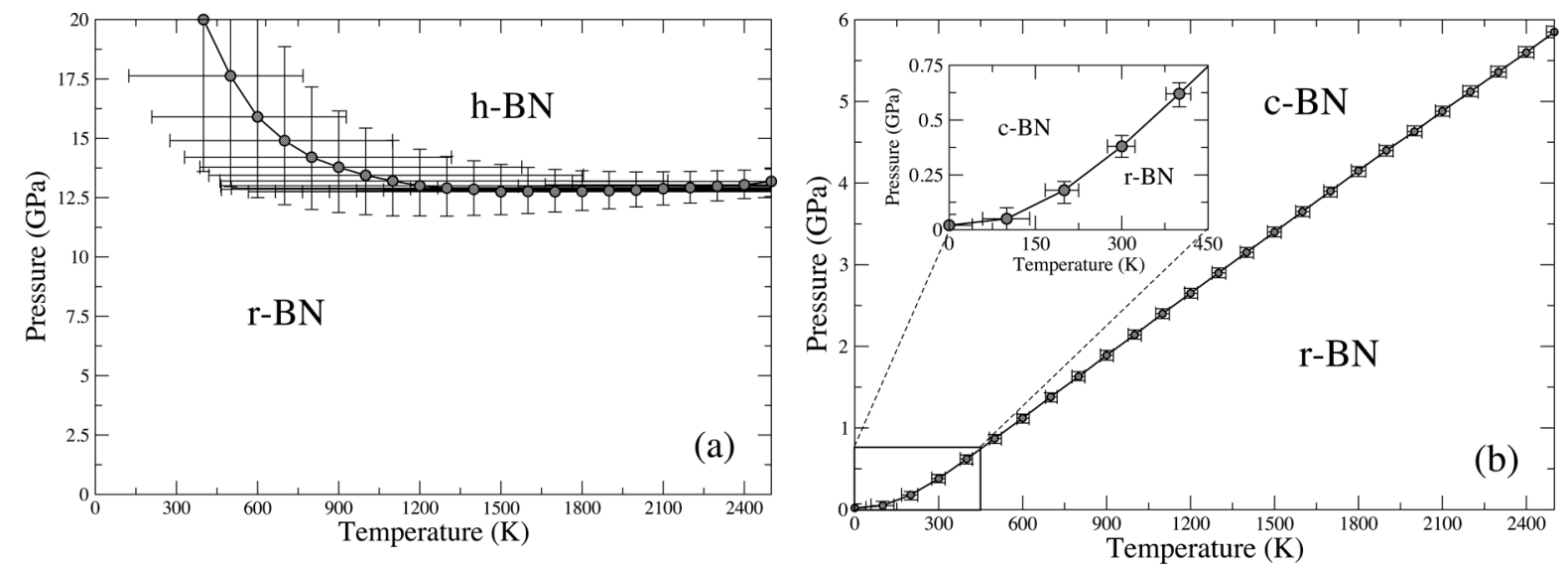

Fig. 1 Calculated p-T phase diagrams at a composition of $\mathrm{BN}$, illustrating (a) the relative thermodynamic stability between r-BN and h-BN and (b) the thermodynamic stability of boron nitride, including the four polytypes, r-BN, h-BN, c-BN, and w-BN. The insert in Fig $1 \mathrm{~b}$ highlights for clarity the stabilities of r-BN and c-BN in the low-temperature and lowpressure region. For completeness are error bars, indicating the consequence of an inherent $\pm 1 \mathrm{meV} /$ atom uncertainty of the numerical parameters in the method, included. 
Fig. 1(b) illustrates the relative thermodynamic stability between the polytypes, $r-$ $\mathrm{BN}, \mathrm{h}-\mathrm{BN}, \mathrm{c}-\mathrm{BN}$, and $\mathrm{w}-\mathrm{BN}$ at $\mathrm{p}$ ranging from 0 to $6 \mathrm{GPa}$. At $\mathrm{T}=0 \mathrm{~K}$ and $\mathrm{p} 0.1 \mathrm{GPa}$ (atmospheric pressure), $\mathrm{r}-\mathrm{BN}$ is suggested to undergo the structural phase transformation to c-BN. At T > $300 \mathrm{~K}$, the r-BN-to-c-BN transition pressure increases almost linearly with increasing temperature seen from $\mathrm{T}=300 \mathrm{~K}$ where the transformation from $\mathrm{r}-\mathrm{BN}$ to c-BN takes place at $\mathrm{p}=0.4 \mathrm{GPa}$, while the transition pressure increases to $5.7 \mathrm{GPa}$ at $\mathrm{T}=$ $2400 \mathrm{~K}$. From these calculations, it is evident that although considered, neither h-BN nor $\mathrm{w}-\mathrm{BN}$ was found to be thermodynamically favored at the investigated temperatures and pressures.

From the phonon density of states (PDOS) of r-BN, h-BN, c-BN and w-BN in (Fig. 2), it is seen that their phonon frequencies are all positive, thus confirming the dynamical stabilities of all polytypes. It is also worth mentioning that our derived PDOS is found to well match those previously reported by Yu et al. ${ }^{30}$ and Kern et al. ${ }^{31}$ in terms of both phonon frequencies and features of corresponding density of states. This strengthens the reliability of our theoretical prediction.

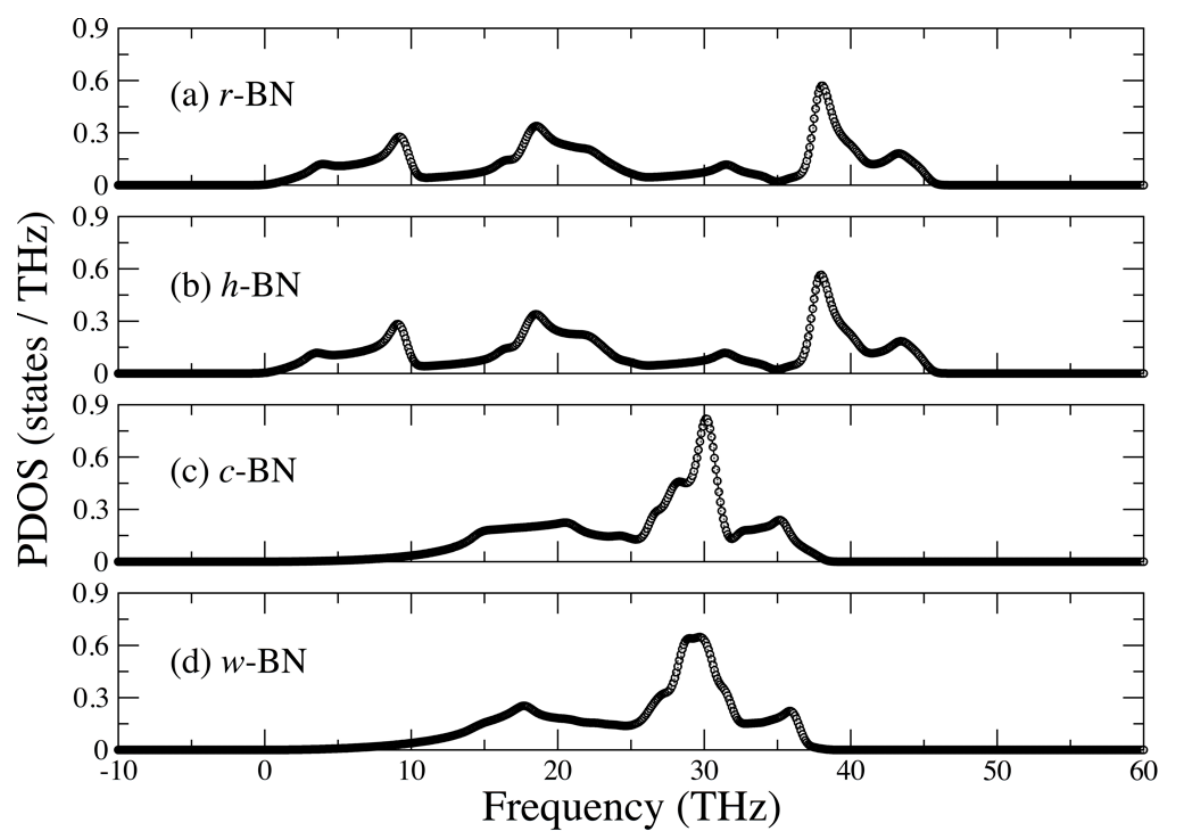

Fig. 2 Phonon density of states (PDOS) of (a) r-BN, (b) h-BN, (c) c-BN, and (d) w-BN. 
The optimized lattice parameters of $r-B N, h-B N$ and $c-B N$ as a function of temperature were derived from the quasiharmonic approximation (Fig. 3). At $\mathrm{T}=300 \mathrm{~K}$, the lattice parameters a and c of r-BN are $2.509 \AA$ and $10.089 \AA$, respectively, and the lattice parameter a of c-BN is $3.623 \AA$. As can be seen from Table 1, our theoretical values derived in the present work are found to differ from the experimental ones, measured at room temperature, by less than $1 \%{ }^{32-34}$. Thus, our theoretical calculations are in good agreement with the experimental data. It is worth noting that as the temperature increases from $300 \mathrm{~K}$ to $2400 \mathrm{~K}$ the isotropic c-BN lattice expands $0.05 \AA$, $(1.3 \%)$ while the anisotropic r-BN and h-BN lattices expands $0.4 \AA(4 \%)$ and $0.03 \AA$ $(0.4 \%)$, respectively in the c-direction, but only $0.004 \AA(0.16 \%)$ and $0.001 \AA(0.04 \%)$, respectively, in the a-direction. This is a signature of strong B-N bonds within the $\mathrm{sp}^{2}$ hybridized basal planes.

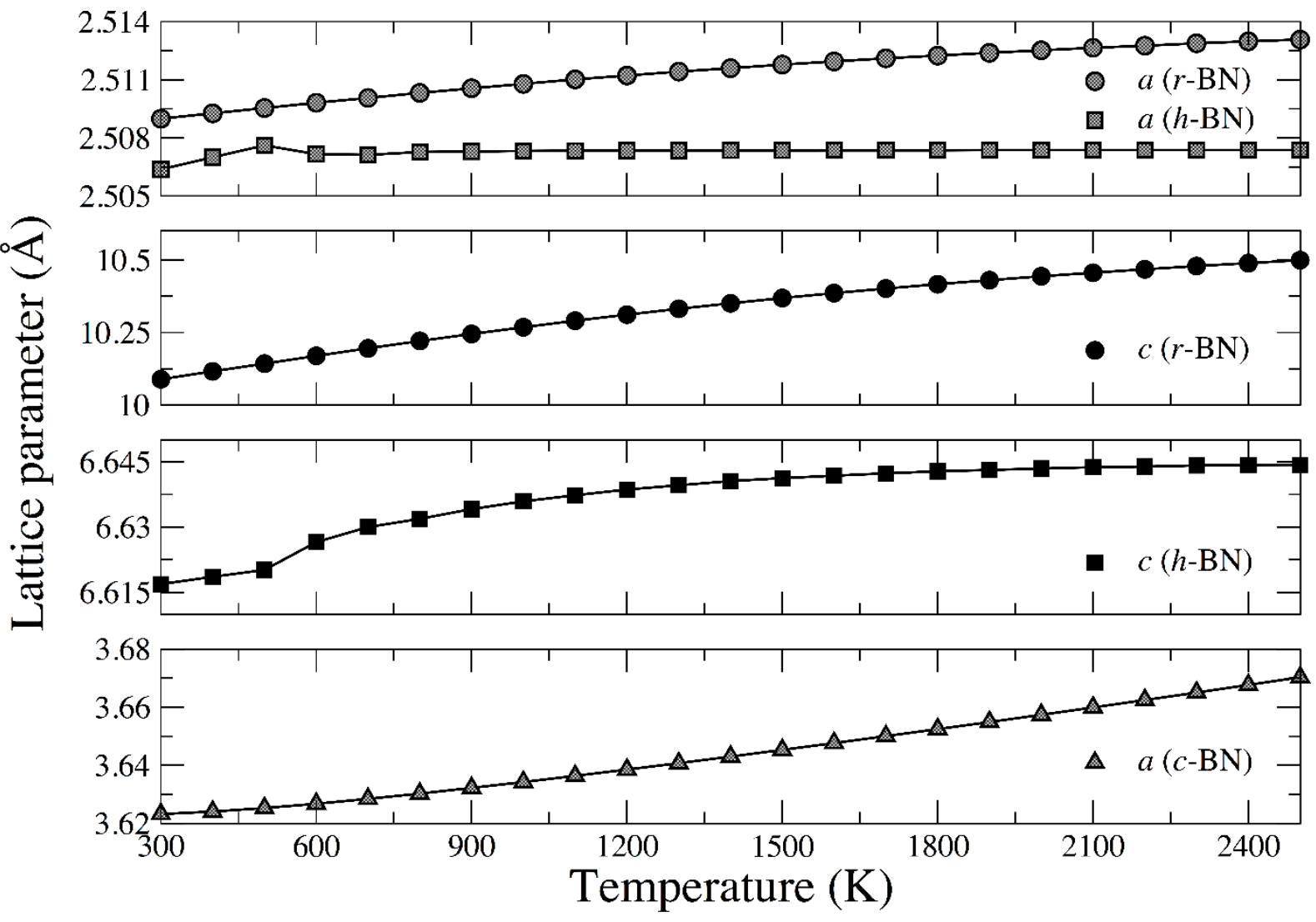

Fig. 3 Optimized lattice parameters of r-BN, h-BN and c-BN as a function of temperature. 
From Fig. 3 an anomalous feature in the lattice parameters of h-BN can be noted at about $600 \mathrm{~K}$, where the lattice parameter $a$ contracts and then barely expands at higher temperature. The contraction may be interpreted as the negative thermal expansion coefficient in the a-axis, previously reported for h-BN. ${ }^{35}$

Table 1 Lattice parameters of r-BN and c-BN at $T=300 \mathrm{~K}$, calculated in the present work. Comparison is made with the existing experimental data, reported in the literature. ${ }^{32-34}$

\begin{tabular}{llll}
\hline & $\mathrm{a}(\AA)$ & $\mathrm{c}(\AA)$ & Reference \\
\hline & 2.509 & 10.089 & This work \\
r-BN & 2.506 & 10.03 & $(32)$ \\
& 2.504 & 9.99 & $(34)$ \\
& & & \\
& 2.506 & 6.617 & This work \\
h-BN & 2.504 & 6.656 & $(34)$ \\
& & & \\
\hline & 3.623 & - & This work \\
c-BN & 3.616 & - & $(33)$ \\
& 3.615 & - & $(34)$ \\
\hline
\end{tabular}

\section{IV.DISCUSSION}

Our calculations show that of the four BN polytypes, $\mathrm{r}-\mathrm{BN}$ is the most thermodynamically stable at typical CVD conditions; $\mathrm{T}=1400-1800 \mathrm{~K}$ and $\mathrm{p}=1000$ 10000 Pa, see Fig. 1(b). This is supported by experimental studies by Solozhenko et al. ${ }^{36}$ The relative thermodynamic stability of the four BN polytypes previously derived from first-principles approach by $\mathrm{Yu}$ et al. ${ }^{30}$ and Solozhenko et al. ${ }^{37}$ suggested c-BN to be the most thermodynamically stable phase at ambient conditions. We attribute this discrepancy to our results to be a consequence of the inclusion of correction factors for long-range van der Waals interactions between the $\mathrm{BN}$ sheets in the $\mathrm{sp}^{2}$-hybridized 
polytypes h-BN and r-BN. These corrections were not included in Ref. (30). Furthermore, it has been demonstrated that standard DFT calculations fail to provide an appropriate description of the van der Waals interactions, which is important for explaining the properties, e.g., interlayer distance and binding energy, of layered materials like graphite $^{38}$ and h-BN ${ }^{39,40}$.

In order to support that the thermodynamic stability of r-BN, predicted in the presented work, does not arise from the use of particular van der Waals correction methods, we evaluate the relative stability between $\mathrm{r}-\mathrm{BN}$ and $\mathrm{h}-\mathrm{BN}$ at $\mathrm{T}=0 \mathrm{~K}$ without the inclusion of the phonon zero-point motion by using different correction methods, i.e., DFT-D2 $2^{41}$ and DFT-TS ${ }^{42,43}$ methods. We find that in all cases $r-B N$ is predicted to be thermodynamically favored over h-BN, indicating that our results are qualitatively robust regardless of which van der Waals correction method is used. Yu et al. ${ }^{30}$ investigated, apart from the relative stability, different possible transformation paths among the four polytypes of boron nitride. They demonstrated that the direct martensitic transformation between $\mathrm{r}-\mathrm{BN}$ and c-BN exhibits the smallest energy barrier. As compared to that of $\mathrm{r}$ $\mathrm{BN}$, the direct martensitic transformation between $\mathrm{h}-\mathrm{BN}$ and $\mathrm{c}-\mathrm{BN}$ is far less favorable, and an intermediate phase, i.e., $\mathrm{r}-\mathrm{BN}$ or $\mathrm{w}-\mathrm{BN}$, is essentially needed to facilitate such a transformation to c-BN. Even though nucleation and growth-type processes should be considered in the transformation studies, nevertheless, their results support the results presented here that $\mathrm{r}-\mathrm{BN}$ and c-BN are the only thermodynamically stable phases of boron nitride, thus appearing on the phase diagram depicted in Fig. 1(b), while h-BN and $\mathrm{w}-\mathrm{BN}$ may merely be metastable polytypes of $\mathrm{BN}$.

The results in this study suggest $\mathrm{r}-\mathrm{BN}$ to be the most favorable BN polytype at typical conditions for CVD of $\mathrm{sp}^{2}$-BN films, $1000-10000 \mathrm{~Pa}$ and $1100-1500{ }^{\circ} \mathrm{C}(1400-$ $1800 \mathrm{~K})$. As thermally activated CVD is at thermal equilibrium, the results presented here yield questions for the conditions at which h-BN can be deposited. The authors have shown that phase determination of 0001 oriented $\mathrm{sp}^{2}-\mathrm{BN}$ films require advanced thin film $\mathrm{X}$-ray diffraction techniques; glancing incidence diffraction of asymmetric peaks with $2 \theta$ angles unique for each structure and pole figure measurements. ${ }^{3}$ The authors have reported CVD of h-BN using $\alpha-\mathrm{Al}_{2} \mathrm{O}_{3}(0001)$ with a strained $\mathrm{AlN}$ buffer layer as substrate at $1500{ }^{\circ} \mathrm{C}$ and $7000 \mathrm{~Pa} .{ }^{44}$ These conditions yielded a polytype pure h-BN film 
of $4 \mathrm{~nm}$ before the h-BN stacking altered to that in r-BN and where r-BN grew to a total film thickness of $200 \mathrm{~nm}$. The change in polytype was suggested to depend on latticemismatch-induced stress relaxation. As comparison, growth on $6 \mathrm{H}-\mathrm{SiC}(0001)$ substrates resulted in a $200 \mathrm{~nm}$ as phase pure r-BN film, using the same CVD process conditions as on $\alpha-\mathrm{Al}_{2} \mathrm{O}_{3}(0001)$.

From our calculations, it seems that the growth of h-BN as a thin film material must be conducted far from thermal equilibrium, highlighting deposition techniques such as sputtering or possibly plasma CVD. From our previously reported growth results, the choice of substrate for h-BN seems critical and the available thickness achievable seems limited. Our calculations indicate that the h-BN lattice seems to be less liable to expansion than the r-BN lattice from our results on thermal expansion of the lattices, indicating that $\mathrm{r}-\mathrm{BN}$ is less sensitive to lattice mismatch to the substrate. All these conditions are met in the study by Sutter et al. ${ }^{45}$, presenting epitaxial growth of h-BN on $\mathrm{Al}_{2} \mathrm{O}_{3}(0001)$ substrates with an in-situ epitaxially grown $100 \mathrm{~nm} \mathrm{Ru}(0001)$ seed layer, using reactive radio frequency magnetron sputtering of a boron target in a nitrogen containing plasma at a temperature of $850^{\circ} \mathrm{C}$. The h-BN films could be deposited to a thickness of approximately 10 BN layers. This corresponds to five unit cells in the cdirection and $3.3 \mathrm{~nm}$, which is close to the $4 \mathrm{~nm}$ reported from growth by CVD by the authors ${ }^{44}$.

\section{SUMMARY}

From van der Waals corrected density functional theory and thermodynamic stability calculations it is found that $\mathrm{r}-\mathrm{BN}$ is the most stable $\mathrm{BN}$ polytype at conditions typically employed for CVD of $\mathrm{sp}^{2}-\mathrm{BN}$ thin films, $1100-1500^{\circ} \mathrm{C}$ and 1000-10 $000 \mathrm{~Pa}$, suggesting that deposition of h-BN films must be done far from thermal equilibrium. It is also found that the h-BN lattice less liable to expansion than the r-BN lattice and therefore likely to be more sensitive to lattice mismatch to the substrate.

\section{Acknowledgements}


This work was supported by the Swedish Foundation for Strategic Research (SSF) (Contract No. IS14-0027). H.P., B.A., and H.H. acknowledge financial support from the Swedish Government Strategic Research Area in Materials Science on Advanced Functional Materials at Linköping University (Faculty Grant SFO-Mat-LiU No. 200900971) B.A. acknowledge financial support from SSF through the Future Research Leaders 6 grant and from the Swedish Research Council (VR) through grant No. 20146336 and by Marie Sklodowska Curie Actions, Cofund, Project INCA 600398. A.E. acknowledges financial support from Kungl. Ingenjörsvetenskapsakademiens Hans Werthén-Fond. All calculations were carried out using supercomputer resources provided by the Swedish National Infrastructure for Computing (SNIC) performed at the National Supercomputer Centre (NSC) and the Center for High Performance Computing (PDC).

(1) R. H. Wentorf, J. Chem. Phys. 26, 956 (1957).

(2) A. Hérold, et al. Compt. Rend., 246, 1866 (1958).

(3) M. Chubarov, H. Högberg, A. Henry and H. Pedersen, J. Vac. Sci. Technol. A 36, 030801 (2018).

(4) Joint Committee on Powder Diffraction Standards, JCPDS, Swarthmore, PA, pattern 34 - 0421; pattern $45-1171$.

(5) H. X. Jiang and J. Y. Lin, Semicond. Sci. Technol. 29, 084003 (2014).

(6) S. Majety, J. Li, W. P. Zhao, B. Huang, S. H. Wei, J. Y. Lin and H. X. Jiang, Appl. Phys. Lett. 102, 213505 (2013).

(7) N. Izyumskaya, D. O. Demchenko, S. Das, Ü. Özgür, V. Avrutin and H. Morkoç, Adv. Electron. Mater. 3, 1600485 (2017).

(8) J. Bao, K. Jeppson, M. Edwards, Y. Fu, L. Ye, X. Lu and J. Liu, Electron. Mater. Lett. 12, 1 (2016).

(9) G. Cassabois, P. Valvin, and B. Gil, Nat. Photonics 10, 262 (2016).

(10) L. Xu, J. Zhan, J. Hu, Y. Bando, X. Yuan, T. Sekiguchi, M. Mitome, and D. Golberg, Adv. Mater. 19, 2141 (2007). 
(11) M. Chubarov, H. Pedersen, H. Högberg, J. Jensen and A. Henry, Cryst. Growth Des. 12, $3215(2012)$

(12) Y. Kobayashi and T. Akasaka, J. Cryst. Growth 310, 5044-5047 (2008).

(13) M. Chubarov, H. Pedersen, H. Högberg, Zs. Czigany and A. Henry, CrystEngComm 16, 5430-5436 (2014).

(14) Q. Paduano, M. Snure, D. Weyburne, A. Kiefer, G. Siegel and J. Hu, J. Cryst. Growth 449, 148-155 (2016).

(15) A. Rice, A. Allerman, M. Crawford, T. Beechem, T. Ohta, C. Spataru, J. Fiegel and M. Smith, J. Cryst. Growth 485, 90-95 (2018).

(16) P. Hohenberg and W. Kohn, Phys. Rev. 136, B864 (1964).

(17) W. Kohn and L. J. Sham, Phys. Rev. 140, A1133 (1965).

(18) P. E. Blöchl, Phys. Rev. B 50, 17953 (1994).

(19) G. Kresse and J. Furthmüller, Comp. Mater. Sci. 6, 15 (1996).

(20) G. Kresse and J. Furthmüller, Phys. Rev. B 54, 11169 (1996).

(21) J. Perdew, K. Burke, and M. Ernzerhof, Phys. Rev. Lett. 77, 3865 (1996).

(22) H. J. Monkhorst and J. D. Pack, Phys. Rev. B 13, 5188 (1976).

(23) S. Grimme, J. Antony, S. Ehrlich, and S. Kreig, J. Chem. Phys. 132, 154104 (2010).

(24) S. Grimme, S. Ehrlich, and L. Goerigk. J. Comp. Chem. 32, 1456 (2011).

(25)A. Togo and I. Tanaka, Scripta Mater. 108, 1 (2015).

(26) A. Togo, F. Oba, and I. Tanaka, Phys. Rev. B 78, 134106 (2008).

(27) K. Parlinski, Z. Q. Li, and Y. Kawazoe, Phys. Rev. Lett. 78, 4063 (1997).

(28) F. D. Murnaghan, Proc. Nat. Acad. Sci. 30, 382 (1944).

(29) F. Birch, Phys. Rev. 71, 809 (1947).

(30) W. J. Yu, W. M. Lau, S. P. Chan, Z. F. Liu, and Q. Q. Zheng, Phys. Rev. B 67, 014108 (2003). 
(31) G. Kern, G. Kresse, and J. Hafner. Phys. Rev. B 59, 8551 (1999).

(32) A. Onodera, K. Inoue, H. Yoshihara, H. Nakae, and T. Matsuda, J. Mater. Sci. 25, 4279 (1990).

(33) V. L. Solozhenko, V. V. Chernyshev, G. V. Fetisov, V. B. Rybakov, and I. A. Petrusha, J. Phys. Chem. Sol. 51, 1011 (1990).

(34) A. V. Kurdyumov, V. L. Solozhenko, and W. B. Zelyavski, J. Appl. Crystallography, 28, 540 (1995).

(35) B. Yates, M. J. Overy and O. Pirgon, Phil. Mag. 32, 847 (1975).

(36) V. L. Solozhenko, I. A. Petrusha and A. A. Svirid, High Press. Res. 15, 95 (1995).

(37) V. L. Solozhenko, V. Z. Turkevich and W. B. Holzapfel, J. Phys. Chem. B 103, 2903 (1999).

(38) I. Mosyagin, D. Gambino, D. G. Sangiovanni, I. A. Abrikosov and N. M Caffrey, Phys. Rev. B 98, 174103 (2018).

(39) N. Marom, J. Bernstein, J. Garel, A. Tkatchenko, E. Joselevich, L. Kronik, and O. Hod, Phys. Rev. Lett. 105, 046801 (2010).

(40) A. Marini, P. García-González, and A. Rubio. Phys. Rev. Lett. 96, 136404 (2006).

(41) S. Grimme, J. Comp. Chem. 27, 1787 (2006).

(42) A. Tkatchenko and M. Scheffler, Phys. Rev. Lett. 102, 073005 (2009).

(43) A. Tkatchenko and R. A. Di Stasio and R. Car and M. Scheffler, Phys. Rev. Lett. 108, 236402 (2012).

(44) M. Chubarov, H. Pedersen, H. Högberg, Zs. Czigány, M. Garbrecht and A. Henry, Chem. Mater. 27, 1640 (2015).

(45) P. Sutter, J. Lahiri, P. Zahl, B. Wang and E. Sutter, Nano Lett. 13, 276 (2013). 


\section{Supplementary Information}

\section{Thermodynamic stability of hexagonal and rhombohedral BN at CVD conditions from van der Waals corrected first principles calculations}

Running title: Stability of h-BN and r-BN at CVD conditions

Running Authors: Pedersen et al.

Henrik Pedersen ${ }^{\text {a) }}$, Björn Alling, Hans Högberg and Annop Ektarawong ${ }^{\text {b) }}$ Department of Physics, Chemistry and Biology, Linköping University, SE-58183 Linköping, Sweden

a) Electronic mail: henrik.pedersen@liu.se

b) Present address: Extreme Condition Physics Research Laboratory, Physics of Energy Materials Research Unit, Department of Physics, Faculty of Science, Chulalongkorn University, Bangkok, 10330, Thailand

\section{Supplementary Figure S1}

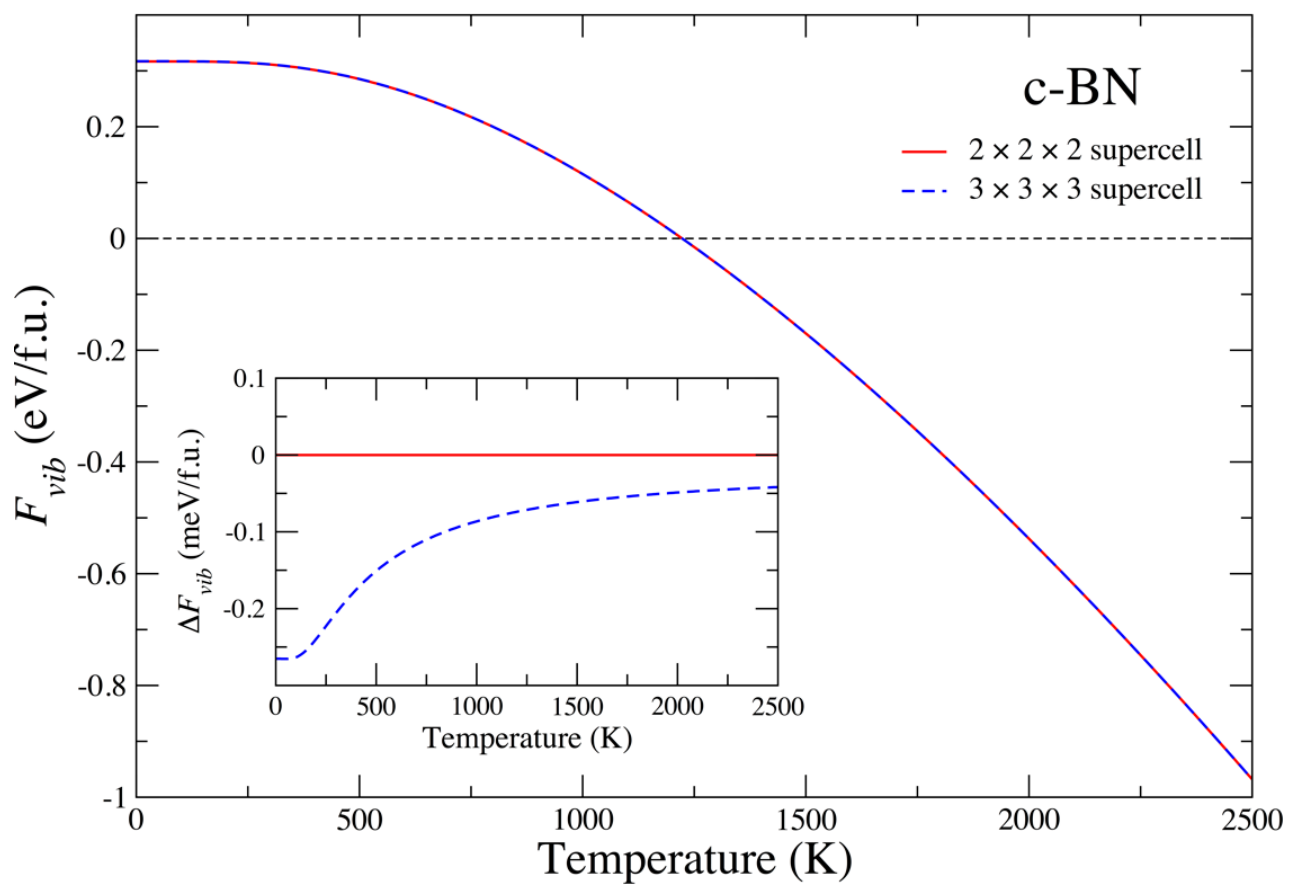

Figure S1: Vibrational free energies $\left(F_{v i b}\right)$ of c-BN, derived from $2 \times 2 \times 2$ (red line) and $3 \times 3 \times 3$ (blue dashed line) conventional cubic unit cells. The inset represents the free energy difference between the two model sizes. 


\section{Supplementary Information: Gibbs free energy difference}

The Gibbs free energy difference of the four polytypes of boron nitride ( $r-\mathrm{BN}, \mathrm{h}-\mathrm{BN}, \mathrm{c}-\mathrm{BN}$, and w-BN) as a function of pressure ranging from 0 to $20 \mathrm{GPa}$, calculated with respect to $\mathrm{r}-\mathrm{BN}$ as a reference at a given temperature of 0 Kelvin. The unit of the Gibbs free energy difference for the four polytypes of boron nitride is given in $\mathrm{eV} /$ atom.

$\begin{array}{lllll}\mathbf{P}(\mathbf{G P a}) \mathbf{r}-\mathbf{B N} & \mathbf{h}-\mathbf{B N} & \mathbf{c}-\mathbf{B N} & \mathbf{w}-\mathbf{B N} \\ 0 & 0 & 0.005286002 & 0.00046129 & 0.017516986 \\ 0.1 & 0 & 0.005213269 & -0.001502839 & 0.015562396 \\ 0.2 & 0 & 0.005142388 & -0.003447442 & 0.013627333 \\ 0.3 & 0 & 0.005073255 & -0.005373243 & 0.011711072 \\ 0.4 & 0 & 0.005005775 & -0.00728091 & 0.009812944 \\ 0.5 & 0 & 0.004939863 & -0.009171063 & 0.007932329 \\ 0.6 & 0 & 0.004875443 & -0.01104428 & 0.00606865 \\ 0.7 & 0 & 0.004812444 & -0.0129011 & 0.004221368 \\ 0.8 & 0 & 0.0047508 & -0.014742025 & 0.00238998 \\ 0.9 & 0 & 0.004690454 & -0.016567528 & 0.000574014 \\ 1 & 0 & 0.00463135 & -0.018378053 & -0.001226975 \\ 1.1 & 0 & 0.004573437 & -0.020174018 & -0.003013404 \\ 1.2 & 0 & 0.004516668 & -0.021955818 & -0.004785669 \\ 1.3 & 0 & 0.004461 & -0.023723827 & -0.006544142 \\ 1.4 & 0 & 0.004406392 & -0.025478398 & -0.008289178 \\ 1.5 & 0 & 0.004352806 & -0.027219866 & -0.010021112 \\ 1.6 & 0 & 0.004300207 & -0.028948551 & -0.011740263 \\ 1.7 & 0 & 0.00424856 & -0.030664755 & -0.013446935 \\ 1.8 & 0 & 0.004197835 & -0.032368769 & -0.015141416 \\ 1.9 & 0 & 0.004148001 & -0.034060868 & -0.016823982 \\ 2 & 0 & 0.004099032 & -0.035741315 & -0.018494898 \\ 2.1 & 0 & 0.004050901 & -0.037410363 & -0.020154415 \\ 2.2 & 0 & 0.004003582 & -0.039068254 & -0.021802775 \\ 2.3 & 0 & 0.003957052 & -0.040715218 & -0.023440209 \\ 2.4 & 0 & 0.003911289 & -0.042351477 & -0.025066939\end{array}$




\begin{tabular}{|c|c|c|c|c|}
\hline .5 & 0 & 0.003866271 & -0.043977246 & -0.026683178 \\
\hline 2.6 & 0 & 0.003821979 & -0.045592728 & -0.028289131 \\
\hline 2.7 & 0 & 0.003778393 & -0.047198121 & -0.029884996 \\
\hline 2.8 & 0 & 0.003735494 & -0.048793615 & -0.031470963 \\
\hline 2.9 & 0 & 0.003693265 & -0.050379392 & -0.033047213 \\
\hline 3 & 0 & 0.00365169 & -0.051955629 & -0.034613924 \\
\hline 3.1 & 0 & 0.003610753 & -0.053522497 & -0.036171265 \\
\hline 3.2 & 0 & 0.003570437 & -0.055080158 & -0.037719402 \\
\hline 3.3 & 0 & 0.003530729 & -0.056628774 & -0.039258492 \\
\hline 3.4 & 0 & 0.003491614 & -0.058168495 & -0.04078869 \\
\hline 3.5 & 0 & 0.00345308 & -0.059699472 & -0.042310144 \\
\hline 3.6 & 0 & 0.003415112 & -0.061221848 & -0.043822997 \\
\hline 3.7 & 0 & 0.003377699 & -0.062735763 & -0.045327389 \\
\hline 3.8 & 0 & 0.003340829 & -0.064241351 & -0.046823456 \\
\hline 3.9 & 0 & 0.00330449 & -0.065738743 & -0.048311327 \\
\hline 4 & 0 & 0.003268671 & -0.067228067 & -0.04979113 \\
\hline 4.1 & 0 & 0.003233362 & -0.068709445 & -0.051262988 \\
\hline 4.2 & 0 & 0.003198552 & -0.070182997 & -0.052727021 \\
\hline 4.3 & 0 & 0.003164231 & -0.071648839 & -0.054183346 \\
\hline 4.4 & 0 & 0.003130389 & -0.073107086 & -0.055632074 \\
\hline 4.5 & 0 & 0.003097019 & -0.074557846 & -0.057073317 \\
\hline 4.6 & 0 & 0.003064109 & -0.076001226 & -0.05850718 \\
\hline 4.7 & 0 & 0.003031653 & -0.07743733 & -0.059933769 \\
\hline 4.8 & 0 & 0.002999641 & -0.07886626 & -0.061353184 \\
\hline 4.9 & 0 & 0.002968066 & -0.080288115 & -0.062765524 \\
\hline 5 & 0 & 0.002936919 & -0.08170299 & -0.064170885 \\
\hline 5.1 & 0 & 0.002906194 & -0.08311098 & -0.065569362 \\
\hline 5.2 & 0 & 0.002875882 & -0.084512174 & -0.066961044 \\
\hline 5.3 & 0 & 0.002845977 & -0.085906664 & -0.068346022 \\
\hline 5.4 & 0 & 0.002816471 & -0.087294535 & -0.069724382 \\
\hline 5.5 & 0 & 0.002787359 & -0.088675873 & -0.071096209 \\
\hline 5.6 & 0 & 0.002758633 & -0.09005076 & -0.072461587 \\
\hline
\end{tabular}




\begin{tabular}{|c|c|c|c|c|}
\hline 5.7 & 0 & 0.002730287 & -0.091419276 & -0.073820595 \\
\hline 5.8 & 0 & 0.002702315 & -0.092781502 & -0.075173313 \\
\hline 5.9 & 0 & 0.002674711 & -0.094137515 & -0.076519818 \\
\hline 6 & 0 & 0.00264747 & -0.095487389 & -0.077860185 \\
\hline 6.1 & 0 & 0.002620585 & -0.096831199 & -0.079194489 \\
\hline 6.2 & 0 & 0.002594051 & -0.098169017 & -0.080522802 \\
\hline 6.3 & 0 & 0.002567863 & -0.099500912 & -0.081845193 \\
\hline 6.4 & 0 & 0.002542015 & -0.100826956 & -0.083161733 \\
\hline 6.5 & 0 & 0.002516503 & -0.102147214 & -0.084472488 \\
\hline 6.6 & 0 & 0.002491322 & -0.103461753 & -0.085777525 \\
\hline 6.7 & 0 & 0.002466466 & -0.104770638 & -0.087076909 \\
\hline 6.8 & 0 & 0.002441931 & -0.106073933 & -0.088370703 \\
\hline 6.9 & 0 & 0.002417713 & -0.107371698 & -0.089658969 \\
\hline 7 & 0 & 0.002393807 & -0.108663997 & -0.090941768 \\
\hline 7.1 & 0 & 0.002370209 & -0.109950887 & -0.09221916 \\
\hline 7.2 & 0 & 0.002346914 & -0.111232428 & -0.093491204 \\
\hline 7.3 & 0 & 0.002323918 & -0.112508676 & -0.094757956 \\
\hline 7.4 & 0 & 0.002301217 & -0.113779689 & -0.096019473 \\
\hline 7.5 & 0 & 0.002278808 & -0.115045521 & -0.09727581 \\
\hline 7.6 & 0 & 0.002256686 & -0.116306226 & -0.098527021 \\
\hline 7.7 & 0 & 0.002234848 & -0.117561858 & -0.09977316 \\
\hline 7.8 & 0 & 0.00221329 & -0.118812469 & -0.101014278 \\
\hline 7.9 & 0 & 0.002192008 & -0.120058109 & -0.102250426 \\
\hline 8 & 0 & 0.002170999 & -0.121298829 & -0.103481656 \\
\hline 8.1 & 0 & 0.002150259 & -0.122534678 & -0.104708015 \\
\hline 8.2 & 0 & 0.002129785 & -0.123765705 & -0.105929553 \\
\hline 8.3 & 0 & 0.002109574 & -0.124991957 & -0.107146317 \\
\hline 8.4 & 0 & 0.002089622 & -0.126213481 & -0.108358354 \\
\hline 8.5 & 0 & 0.002069927 & -0.127430323 & -0.109565709 \\
\hline 8.6 & 0 & 0.002050485 & -0.128642528 & -0.110768429 \\
\hline 8.7 & 0 & 0.002031293 & -0.12985014 & -0.111966556 \\
\hline 8.8 & 0 & 0.002012348 & -0.131053203 & -0.113160135 \\
\hline
\end{tabular}




\begin{tabular}{|c|c|c|c|c|}
\hline .9 & 0 & 0.001993648 & -0.13225176 & -0.114349209 \\
\hline 9 & 0 & 0.001975189 & -0.133445853 & -0.11553382 \\
\hline 9.1 & 0 & 0.001956968 & -0.134635523 & -0.116714008 \\
\hline 9.2 & 0 & 0.001938984 & -0.13582081 & -0.117889816 \\
\hline 9.3 & 0 & 0.001921233 & -0.137001757 & -0.119061283 \\
\hline 9.4 & 0 & 0.001903712 & -0.1381784 & -0.120228448 \\
\hline 9.5 & 0 & 0.001886419 & -0.13935078 & -0.12139135 \\
\hline 9.6 & 0 & 0.001869352 & -0.140518935 & -0.122550028 \\
\hline 9.7 & 0 & 0.001852508 & -0.141682901 & -0.123704519 \\
\hline 9.8 & 0 & 0.001835884 & -0.142842717 & -0.124854861 \\
\hline 9.9 & 0 & 0.001819478 & -0.143998419 & -0.126001088 \\
\hline 10 & 0 & 0.001803289 & -0.145150042 & -0.127143238 \\
\hline 10.1 & 0 & 0.001787312 & -0.146297622 & -0.128281346 \\
\hline 10.2 & 0 & 0.001771548 & -0.147441193 & -0.129415447 \\
\hline 10.3 & 0 & 0.001755991 & -0.14858079 & -0.130545574 \\
\hline 10.4 & 0 & 0.001740642 & -0.149716447 & -0.131671762 \\
\hline 10.5 & 0 & 0.001725498 & -0.150848197 & -0.132794043 \\
\hline 10.6 & 0 & 0.001710556 & -0.151976072 & -0.133912452 \\
\hline 10.7 & 0 & 0.001695814 & -0.153100106 & -0.135027019 \\
\hline 10.8 & 0 & 0.001681272 & -0.154220328 & -0.136137776 \\
\hline 10.9 & 0 & 0.001666925 & -0.155336772 & -0.137244756 \\
\hline 11 & 0 & 0.001652773 & -0.156449468 & -0.138347989 \\
\hline 11.1 & 0 & 0.001638814 & -0.157558447 & -0.139447505 \\
\hline 11.2 & 0 & 0.001625045 & -0.158663738 & -0.140543335 \\
\hline 11.3 & 0 & 0.001611465 & -0.159765371 & -0.141635507 \\
\hline 11.4 & 0 & 0.001598073 & -0.160863375 & -0.142724052 \\
\hline 11.5 & 0 & 0.001584865 & -0.16195778 & -0.143808998 \\
\hline 11.6 & 0 & 0.001571841 & -0.163048612 & -0.144890374 \\
\hline 11.7 & 0 & 0.001558998 & -0.164135902 & -0.145968207 \\
\hline 11.8 & 0 & 0.001546336 & -0.165219676 & -0.147042526 \\
\hline 11.9 & 0 & 0.001533851 & -0.166299961 & -0.148113357 \\
\hline 12 & 0 & 0.001521544 & -0.167376784 & -0.149180727 \\
\hline
\end{tabular}




\begin{tabular}{|c|c|c|c|c|}
\hline 2.1 & 0 & 0.001509411 & -0.168450173 & -0.150244663 \\
\hline 12.2 & 0 & 0.001497451 & -0.169520153 & -0.151305192 \\
\hline 12.3 & 0 & 0.001485663 & -0.170586749 & -0.152362338 \\
\hline 12.4 & 0 & 0.001474046 & -0.171649988 & -0.153416128 \\
\hline 12.5 & 0 & 0.001462597 & -0.172709895 & -0.154466587 \\
\hline 12.6 & 0 & 0.001451315 & -0.173766494 & -0.15551374 \\
\hline 12.7 & 0 & 0.001440199 & -0.174819811 & -0.15655761 \\
\hline 12.8 & 0 & 0.001429247 & -0.175869869 & -0.157598223 \\
\hline 12.9 & 0 & 0.001418458 & -0.176916692 & -0.158635603 \\
\hline 13 & 0 & 0.00140783 & -0.177960304 & -0.159669772 \\
\hline 13.1 & 0 & 0.001397362 & -0.179000729 & -0.160700755 \\
\hline 13.2 & 0 & 0.001387052 & -0.180037988 & -0.161728574 \\
\hline 13.3 & 0 & 0.0013769 & -0.181072106 & -0.162753252 \\
\hline 13.4 & 0 & 0.001366903 & -0.182103105 & -0.163774812 \\
\hline 13.5 & 0 & 0.001357061 & -0.183131006 & -0.164793276 \\
\hline 13.6 & 0 & 0.001347372 & -0.184155832 & -0.165808666 \\
\hline 13.7 & 0 & 0.001337835 & -0.185177605 & -0.166821003 \\
\hline 13.8 & 0 & 0.001328448 & -0.186196345 & -0.167830309 \\
\hline 13.9 & 0 & 0.001319211 & -0.187212074 & -0.168836606 \\
\hline 14 & 0 & 0.001310122 & -0.188224814 & -0.169839913 \\
\hline 14.1 & 0 & 0.00130118 & -0.189234584 & -0.170840252 \\
\hline 14.2 & 0 & 0.001292384 & -0.190241405 & -0.171837644 \\
\hline 14.3 & 0 & 0.001283732 & -0.191245297 & -0.172832107 \\
\hline 14.4 & 0 & 0.001275223 & -0.192246281 & -0.173823664 \\
\hline 14.5 & 0 & 0.001266857 & -0.193244375 & -0.174812332 \\
\hline 14.6 & 0 & 0.001258632 & -0.1942396 & -0.175798131 \\
\hline 14.7 & 0 & 0.001250546 & -0.195231975 & -0.176781082 \\
\hline 14.8 & 0 & 0.0012426 & -0.196221519 & -0.177761203 \\
\hline 14.9 & 0 & 0.001234791 & -0.19720825 & -0.178738512 \\
\hline 15 & 0 & 0.001227119 & -0.198192188 & -0.179713029 \\
\hline 15.1 & 0 & 0.001219583 & -0.19917335 & -0.180684772 \\
\hline 15.2 & 0 & 0.001212181 & -0.200151756 & -0.181653759 \\
\hline
\end{tabular}




\begin{tabular}{|c|c|c|c|c|}
\hline 15.3 & 0 & 0.001204913 & -0.201127422 & -0.182620008 \\
\hline 15.4 & 0 & 0.001197777 & -0.202100368 & -0.183583538 \\
\hline 15.5 & 0 & 0.001190773 & -0.203070609 & -0.184544365 \\
\hline 15.6 & 0 & 0.001183899 & -0.204038165 & -0.185502507 \\
\hline 15.7 & 0 & 0.001177154 & -0.205003053 & -0.186457981 \\
\hline 15.8 & 0 & 0.001170539 & -0.205965288 & -0.187410805 \\
\hline 15.9 & 0 & 0.00116405 & -0.206924889 & -0.188360995 \\
\hline 16 & 0 & 0.001157689 & -0.207881871 & -0.189308569 \\
\hline 16.1 & 0 & 0.001151453 & -0.208836252 & -0.190253542 \\
\hline 16.2 & 0 & 0.001145342 & -0.209788048 & -0.191195931 \\
\hline 16.3 & 0 & 0.001139355 & -0.210737275 & -0.192135752 \\
\hline 16.4 & 0 & 0.001133491 & -0.211683949 & -0.193073022 \\
\hline 16.5 & 0 & 0.001127749 & -0.212628086 & -0.194007756 \\
\hline 16.6 & 0 & 0.001122128 & -0.213569702 & -0.194939969 \\
\hline 16.7 & 0 & 0.001116627 & -0.214508811 & -0.195869678 \\
\hline 16.8 & 0 & 0.001111246 & -0.215445431 & -0.196796898 \\
\hline 16.9 & 0 & 0.001105983 & -0.216379575 & -0.197721643 \\
\hline 17 & 0 & 0.001100839 & -0.217311259 & -0.19864393 \\
\hline 17.1 & 0 & 0.001095811 & -0.218240497 & -0.199563772 \\
\hline 17.2 & 0 & 0.001090899 & -0.219167305 & -0.200481185 \\
\hline 17.3 & 0 & 0.001086103 & -0.220091698 & -0.201396184 \\
\hline 17.4 & 0 & 0.001081421 & -0.221013689 & -0.202308782 \\
\hline 17.5 & 0 & 0.001076853 & -0.221933292 & -0.203218995 \\
\hline 17.6 & 0 & 0.001072398 & -0.222850523 & -0.204126836 \\
\hline 17.7 & 0 & 0.001068055 & -0.223765395 & -0.205032319 \\
\hline 17.8 & 0 & 0.001063823 & -0.224677923 & -0.205935459 \\
\hline 17.9 & 0 & 0.001059703 & -0.225588119 & -0.206836269 \\
\hline 18 & 0 & 0.001055692 & -0.226495998 & -0.207734763 \\
\hline 18.1 & 0 & 0.00105179 & -0.227401572 & -0.208630954 \\
\hline 18.2 & 0 & 0.001047996 & -0.228304857 & -0.209524855 \\
\hline 18.3 & 0 & 0.001044311 & -0.229205864 & -0.210416481 \\
\hline 18.4 & 0 & 0.001040732 & -0.230104606 & -0.211305843 \\
\hline
\end{tabular}




$\begin{array}{lllll}18.5 & 0 & 0.00103726 & -0.231001098 & -0.212192956 \\ 18.6 & 0 & 0.001033893 & -0.231895351 & -0.213077832 \\ 18.7 & 0 & 0.001030631 & -0.232787379 & -0.213960483 \\ 18.8 & 0 & 0.001027474 & -0.233677194 & -0.214840923 \\ 18.9 & 0 & 0.00102442 & -0.234564809 & -0.215719164 \\ 19 & 0 & 0.001021469 & -0.235450236 & -0.216595218 \\ 19.1 & 0 & 0.00101862 & -0.236333487 & -0.217469098 \\ 19.2 & 0 & 0.001015872 & -0.237214575 & -0.218340816 \\ 19.3 & 0 & 0.001013226 & -0.238093511 & -0.219210384 \\ 19.4 & 0 & 0.00101068 & -0.238970308 & -0.220077814 \\ 19.5 & 0 & 0.001008233 & -0.239844978 & -0.220943117 \\ 19.6 & 0 & 0.001005886 & -0.240717532 & -0.221806306 \\ 19.7 & 0 & 0.001003637 & -0.241587982 & -0.222667392 \\ 19.8 & 0 & 0.001001485 & -0.242456339 & -0.223526387 \\ 19.9 & 0 & 0.000999431 & -0.243322615 & -0.224383303 \\ 20 & 0 & 0.000997474 & -0.244186822 & -0.225238149\end{array}$

The Gibbs free energy difference of the four polytypes of boron nitride ( $\mathrm{r}-\mathrm{BN}, \mathrm{h}-\mathrm{BN}, \mathrm{c}-\mathrm{BN}$, and w-BN) as a function of pressure ranging from 0 to $20 \mathrm{GPa}$, calculated with respect to $\mathrm{r}-\mathrm{BN}$ as a reference at a given temperature of $\mathbf{1 0 0}$ Kelvin. The unit of the Gibbs free energy difference for the four polytypes of boron nitride is given in eV/atom.

$\begin{array}{lllll}\mathbf{P}(\mathbf{G P a}) & \mathbf{r}-\mathbf{B N} & \mathbf{h}-\mathbf{B N} & \mathbf{c}-\mathbf{B N} & \mathbf{w}-\mathbf{B N} \\ 0 & 0 & 0.005596475 & 0.000995734 & 0.018050315 \\ 0.1 & 0 & 0.005517683 & -0.000974767 & 0.016089317 \\ 0.2 & 0 & 0.005440904 & -0.002925571 & 0.014148014 \\ 0.3 & 0 & 0.005366022 & -0.004857413 & 0.012225673 \\ 0.4 & 0 & 0.005292936 & -0.006770972 & 0.010321616 \\ 0.5 & 0 & 0.005221553 & -0.008666876 & 0.008435213 \\ 0.6 & 0 & 0.005151789 & -0.01054571 & 0.006565879 \\ 0.7 & 0 & 0.005083566 & -0.012408018 & 0.00471307 \\ 0.8 & 0 & 0.005016814 & -0.014254312 & 0.002876275 \\ 0.9 & 0 & 0.004951469 & -0.016085068 & 0.001055017\end{array}$




\begin{tabular}{|c|c|c|c|c|}
\hline 1 & 0 & 0.004887471 & -0.017900737 & -0.000751154 \\
\hline 1.1 & 0 & 0.004824766 & -0.019701742 & -0.002542662 \\
\hline 1.2 & 0 & 0.004763301 & -0.021488481 & -0.004319905 \\
\hline 1.3 & 0 & 0.004703029 & -0.023261333 & -0.00608326 \\
\hline 1.4 & 0 & 0.004643907 & -0.025020654 & -0.007833086 \\
\hline 1.5 & 0 & 0.004585891 & -0.026766785 & -0.009569722 \\
\hline 1.6 & 0 & 0.004528945 & -0.028500046 & -0.011293489 \\
\hline 1.7 & 0 & 0.004473032 & -0.030220746 & -0.013004695 \\
\hline 1.8 & 0 & 0.004418117 & -0.031929175 & -0.014703631 \\
\hline 1.9 & 0 & 0.004364168 & -0.033625613 & -0.016390577 \\
\hline 2 & 0 & 0.004311154 & -0.035310326 & -0.018065798 \\
\hline 2.1 & 0 & 0.004259049 & -0.036983568 & -0.019729549 \\
\hline 2.2 & 0 & 0.004207824 & -0.038645583 & -0.021382074 \\
\hline 2.3 & 0 & 0.004157453 & -0.040296606 & -0.023023606 \\
\hline 2.4 & 0 & 0.004107912 & -0.041936858 & -0.02465437 \\
\hline 2.5 & 0 & 0.004059179 & -0.043566557 & -0.02627458 \\
\hline 2.6 & 0 & 0.004011231 & -0.045185908 & -0.027884443 \\
\hline 2.7 & 0 & 0.003964048 & -0.046795111 & -0.029484158 \\
\hline 2.8 & 0 & 0.003917609 & -0.048394357 & -0.031073917 \\
\hline 2.9 & 0 & 0.003871895 & -0.04998383 & -0.032653904 \\
\hline 3 & 0 & 0.003826888 & -0.051563708 & -0.034224297 \\
\hline 3.1 & 0 & 0.003782572 & -0.053134164 & -0.035785268 \\
\hline 3.2 & 0 & 0.003738929 & -0.054695362 & -0.037336981 \\
\hline 3.3 & 0 & 0.003695944 & -0.056247462 & -0.038879599 \\
\hline 3.4 & 0 & 0.0036536 & -0.057790621 & -0.040413274 \\
\hline 3.5 & 0 & 0.003611884 & -0.059324986 & -0.041938157 \\
\hline 3.6 & 0 & 0.003570782 & -0.060850704 & -0.043454394 \\
\hline 3.7 & 0 & 0.00353028 & -0.062367914 & -0.044962123 \\
\hline 3.8 & 0 & 0.003490366 & -0.063876753 & -0.046461482 \\
\hline 3.9 & 0 & 0.003451026 & -0.065377352 & -0.047952602 \\
\hline 4 & 0 & 0.003412248 & -0.06686984 & -0.049435611 \\
\hline 4.1 & 0 & 0.003374022 & -0.068354341 & -0.050910634 \\
\hline
\end{tabular}




\begin{tabular}{|c|c|c|c|c|}
\hline & 0 & 0.003336336 & -0.069830975 & -0.05237779 \\
\hline 4.3 & 0 & 0.003299179 & -0.071299859 & -0.053837198 \\
\hline 4.4 & 0 & 0.003262541 & -0.072761108 & -0.055288971 \\
\hline 15 & 0 & 0.003226412 & -0.074214831 & -0.056733219 \\
\hline 4.6 & 0 & 0.003190782 & -0.075661138 & -0.058170051 \\
\hline 4.7 & 0 & 0.003155642 & -0.077100132 & -0.059599571 \\
\hline 4.8 & 0 & 0.003120983 & -0.078531915 & -0.061021882 \\
\hline 4.9 & 0 & 0.003086796 & -0.079956588 & -0.062437082 \\
\hline 5 & 0 & 0.003053073 & -0.081374246 & -0.063845269 \\
\hline 5.1 & 0 & 0.003019804 & -0.082784984 & -0.065246536 \\
\hline 5.2 & 0 & 0.002986984 & -0.084188894 & -0.066640976 \\
\hline 5.3 & 0 & 0.002954603 & -0.085586066 & -0.068028679 \\
\hline 5.4 & 0 & 0.002922654 & -0.086976588 & -0.069409732 \\
\hline 5.5 & 0 & 0.00289113 & -0.088360544 & -0.07078422 \\
\hline 5.6 & 0 & 0.002860024 & -0.089738018 & -0.072152227 \\
\hline 5.7 & 0 & 0.002829329 & -0.091109091 & -0.073513834 \\
\hline 5.8 & 0 & 0.002799038 & -0.092473844 & -0.074869121 \\
\hline 5.9 & 0 & 0.002769146 & -0.093832353 & -0.076218166 \\
\hline 6 & 0 & 0.002739645 & -0.095184696 & -0.077561044 \\
\hline 6.1 & 0 & 0.002710529 & -0.096530945 & -0.078897829 \\
\hline 6.2 & 0 & 0.002681793 & -0.097871173 & -0.080228596 \\
\hline 6.3 & 0 & 0.002653431 & -0.099205452 & -0.081553413 \\
\hline 6.4 & 0 & 0.002625437 & -0.100533852 & -0.082872351 \\
\hline 6.5 & 0 & 0.002597806 & -0.101856439 & -0.084185479 \\
\hline 6.6 & 0 & 0.002570532 & -0.103173282 & -0.085492862 \\
\hline 6.7 & 0 & 0.00254361 & -0.104484444 & -0.086794565 \\
\hline 6.8 & 0 & 0.002517036 & -0.10578999 & -0.088090653 \\
\hline 6.9 & 0 & 0.002490803 & -0.107089981 & -0.089381188 \\
\hline 7 & 0 & 0.002464907 & -0.108384481 & -0.090666231 \\
\hline 7.1 & 0 & 0.002439344 & -0.109673548 & -0.091945843 \\
\hline 7.2 & 0 & 0.00241411 & -0.110957241 & -0.093220082 \\
\hline 7.3 & 0 & 0.002389198 & -0.112235619 & -0.094489005 \\
\hline
\end{tabular}




\begin{tabular}{|c|c|c|c|c|}
\hline .4 & 0 & 0.002364606 & -0.113508737 & -0.095752671 \\
\hline 7.5 & 0 & 0.002340328 & -0.114776652 & -0.097011133 \\
\hline 7.6 & 0 & 0.002316361 & -0.116039417 & -0.098264446 \\
\hline 7.7 & 0 & 0.002292701 & -0.117297086 & -0.099512665 \\
\hline 7.8 & 0 & 0.002269343 & -0.118549711 & -0.100755841 \\
\hline 7.9 & 0 & 0.002246283 & -0.119797344 & -0.101994025 \\
\hline 8 & 0 & 0.002223519 & -0.121040036 & -0.103227269 \\
\hline 8.1 & 0 & 0.002201046 & -0.122277836 & -0.104455621 \\
\hline 8.2 & 0 & 0.00217886 & -0.123510792 & -0.105679131 \\
\hline 8.3 & 0 & 0.002156957 & -0.124738953 & -0.106897846 \\
\hline 8.4 & 0 & 0.002135336 & -0.125962365 & -0.108111814 \\
\hline 8.5 & 0 & 0.002113991 & -0.127181075 & -0.10932108 \\
\hline 8.6 & 0 & 0.00209292 & -0.128395128 & -0.11052569 \\
\hline 8.7 & 0 & 0.002072119 & -0.129604569 & -0.111725689 \\
\hline 8.8 & 0 & 0.002051585 & -0.130809441 & -0.112921119 \\
\hline 8.9 & 0 & 0.002031316 & -0.132009787 & -0.114112025 \\
\hline 9 & 0 & 0.002011307 & -0.133205651 & -0.115298449 \\
\hline 9.1 & 0 & 0.001991555 & -0.134397073 & -0.116480433 \\
\hline 9.2 & 0 & 0.001972059 & -0.135584094 & -0.117658016 \\
\hline 9.3 & 0 & 0.001952815 & -0.136766756 & -0.118831241 \\
\hline 9.4 & 0 & 0.00193382 & -0.137945097 & -0.120000146 \\
\hline 9.5 & 0 & 0.001915071 & -0.139119156 & -0.12116477 \\
\hline 9.6 & 0 & 0.001896565 & -0.140288973 & -0.122325152 \\
\hline 9.7 & 0 & 0.001878301 & -0.141454584 & -0.12348133 \\
\hline 9.8 & 0 & 0.001860275 & -0.142616027 & -0.124633341 \\
\hline 9.9 & 0 & 0.001842484 & -0.143773339 & -0.125781221 \\
\hline 10 & 0 & 0.001824927 & -0.144926555 & -0.126925006 \\
\hline 10.1 & 0 & 0.001807601 & -0.146075711 & -0.128064733 \\
\hline 10.2 & 0 & 0.001790502 & -0.147220843 & -0.129200436 \\
\hline 10.3 & 0 & 0.00177363 & -0.148361984 & -0.130332149 \\
\hline 10.4 & 0 & 0.00175698 & -0.149499169 & -0.131459906 \\
\hline 10.5 & 0 & 0.001740552 & -0.150632431 & -0.132583742 \\
\hline
\end{tabular}




\begin{tabular}{|c|c|c|c|c|}
\hline 10.6 & 0 & 0.001724343 & -0.151761802 & -0.133703688 \\
\hline 10.7 & 0 & 0.00170835 & -0.152887316 & -0.134819777 \\
\hline 10.8 & 0 & 0.001692572 & -0.154009004 & -0.135932042 \\
\hline 10.9 & 0 & 0.001677006 & -0.155126898 & -0.137040513 \\
\hline 11 & 0 & 0.00166165 & -0.156241029 & -0.138145222 \\
\hline 11.1 & 0 & 0.001646502 & -0.157351427 & -0.1392462 \\
\hline 11.2 & 0 & 0.00163156 & -0.158458123 & -0.140343476 \\
\hline 11.3 & 0 & 0.001616822 & -0.159561146 & -0.14143708 \\
\hline 11.4 & 0 & 0.001602286 & -0.160660527 & -0.142527043 \\
\hline 11.5 & 0 & 0.001587949 & -0.161756293 & -0.143613392 \\
\hline 11.6 & 0 & 0.001573812 & -0.162848473 & -0.144696156 \\
\hline 11.7 & 0 & 0.00155987 & -0.163937096 & -0.145775364 \\
\hline 11.8 & 0 & 0.001546122 & -0.165022189 & -0.146851043 \\
\hline 11.9 & 0 & 0.001532567 & -0.16610378 & -0.14792322 \\
\hline 12 & 0 & 0.001519203 & -0.167181896 & -0.148991924 \\
\hline 12.1 & 0 & 0.001506028 & -0.168256563 & -0.150057179 \\
\hline 12.2 & 0 & 0.00149304 & -0.169327808 & -0.151119014 \\
\hline 12.3 & 0 & 0.001480237 & -0.170395656 & -0.152177453 \\
\hline 12.4 & 0 & 0.001467619 & -0.171460134 & -0.153232522 \\
\hline 12.5 & 0 & 0.001455182 & -0.172521267 & -0.154284247 \\
\hline 12.6 & 0 & 0.001442926 & -0.173579079 & -0.155332653 \\
\hline 12.7 & 0 & 0.001430849 & -0.174633596 & -0.156377764 \\
\hline 12.8 & 0 & 0.001418949 & -0.175684841 & -0.157419605 \\
\hline 12.9 & 0 & 0.001407224 & -0.17673284 & -0.158458199 \\
\hline 13 & 0 & 0.001395675 & -0.177777615 & -0.159493572 \\
\hline 13.1 & 0 & 0.001384297 & -0.178819189 & -0.160525745 \\
\hline 13.2 & 0 & 0.001373091 & -0.179857587 & -0.161554742 \\
\hline 13.3 & 0 & 0.001362054 & -0.180892831 & -0.162580586 \\
\hline 13.4 & 0 & 0.001351186 & -0.181924944 & -0.1636033 \\
\hline 13.5 & 0 & 0.001340485 & -0.182953948 & -0.164622906 \\
\hline 13.6 & 0 & 0.001329949 & -0.183979864 & -0.165639425 \\
\hline 13.7 & 0 & 0.001319577 & -0.185002716 & -0.166652881 \\
\hline
\end{tabular}




\begin{tabular}{|c|c|c|c|c|}
\hline 13.8 & 0 & 0.001309367 & -0.186022523 & -0.167663294 \\
\hline 13.9 & 0 & 0.001299319 & -0.187039309 & -0.168670685 \\
\hline 14 & 0 & 0.001289431 & -0.188053093 & -0.169675076 \\
\hline 14.1 & 0 & 0.001279701 & -0.189063896 & -0.170676488 \\
\hline 14.2 & 0 & 0.001270129 & -0.190071739 & -0.17167494 \\
\hline 14.3 & 0 & 0.001260713 & -0.191076643 & -0.172670454 \\
\hline 14.4 & 0 & 0.001251452 & -0.192078627 & -0.173663049 \\
\hline 14.5 & 0 & 0.001242344 & -0.193077711 & -0.174652745 \\
\hline 14.6 & 0 & 0.001233389 & -0.194073914 & -0.175639562 \\
\hline 14.7 & 0 & 0.001224584 & -0.195067257 & -0.176623519 \\
\hline 14.8 & 0 & 0.00121593 & -0.196057758 & -0.177604635 \\
\hline 14.9 & 0 & 0.001207424 & -0.197045436 & -0.178582929 \\
\hline 15 & 0 & 0.001199066 & -0.19803031 & -0.17955842 \\
\hline 15.1 & 0 & 0.001190854 & -0.199012398 & -0.180531127 \\
\hline 15.2 & 0 & 0.001182788 & -0.19999172 & -0.181501067 \\
\hline 15.3 & 0 & 0.001174865 & -0.200968292 & -0.18246826 \\
\hline 15.4 & 0 & 0.001167086 & -0.201942133 & -0.183432722 \\
\hline 15.5 & 0 & 0.001159449 & -0.20291326 & -0.184394472 \\
\hline 15.6 & 0 & 0.001151952 & -0.203881692 & -0.185353527 \\
\hline 15.7 & 0 & 0.001144596 & -0.204847445 & -0.186309904 \\
\hline 15.8 & 0 & 0.001137378 & -0.205810536 & -0.187263621 \\
\hline 15.9 & 0 & 0.001130298 & -0.206770983 & -0.188214694 \\
\hline 16 & 0 & 0.001123355 & -0.207728802 & -0.189163141 \\
\hline 16.1 & 0 & 0.001116548 & -0.20868401 & -0.190108978 \\
\hline 16.2 & 0 & 0.001109875 & -0.209636624 & -0.191052221 \\
\hline 16.3 & 0 & 0.001103336 & -0.210586659 & -0.191992887 \\
\hline 16.4 & 0 & 0.001096929 & -0.211534132 & -0.192930991 \\
\hline 16.5 & 0 & 0.001090655 & -0.212479059 & -0.19386655 \\
\hline 16.6 & 0 & 0.001084511 & -0.213421455 & -0.19479958 \\
\hline 16.7 & 0 & 0.001078497 & -0.214361336 & -0.195730096 \\
\hline 16.8 & 0 & 0.001072612 & -0.215298717 & -0.196658114 \\
\hline 16.9 & 0 & 0.001066856 & -0.216233615 & -0.197583648 \\
\hline
\end{tabular}




\begin{tabular}{|c|c|c|c|c|}
\hline 17 & 0 & 0.001061226 & -0.217166043 & -0.198506714 \\
\hline 17.1 & 0 & 0.001055723 & -0.218096017 & -0.199427327 \\
\hline 17.2 & 0 & 0.001050345 & -0.219023552 & -0.200345502 \\
\hline 17.3 & 0 & 0.001045091 & -0.219948662 & -0.201261254 \\
\hline 17.4 & 0 & 0.001039961 & -0.220871362 & -0.202174596 \\
\hline 17.5 & 0 & 0.001034954 & -0.221791667 & -0.203085544 \\
\hline 17.6 & 0 & 0.001030069 & -0.22270959 & -0.203994112 \\
\hline 17.7 & 0 & 0.001025305 & -0.223625146 & -0.204900313 \\
\hline 17.8 & 0 & 0.001020661 & -0.224538349 & -0.205804162 \\
\hline 17.9 & 0 & 0.001016137 & -0.225449212 & -0.206705673 \\
\hline 18 & 0 & 0.001011731 & -0.226357749 & -0.207604859 \\
\hline 18.1 & 0 & 0.001007443 & -0.227263975 & -0.208501735 \\
\hline 18.2 & 0 & 0.001003273 & -0.228167901 & -0.209396312 \\
\hline 18.3 & 0 & 0.000999218 & -0.229069542 & -0.210288605 \\
\hline 18.4 & 0 & 0.000995279 & -0.229968911 & -0.211178627 \\
\hline 18.5 & 0 & 0.000991455 & -0.230866021 & -0.212066391 \\
\hline 18.6 & 0 & 0.000987745 & -0.231760884 & -0.212951909 \\
\hline 18.7 & 0 & 0.000984148 & -0.232653514 & -0.213835196 \\
\hline 18.8 & 0 & 0.000980663 & -0.233543924 & -0.214716263 \\
\hline 18.9 & 0 & 0.000977291 & -0.234432125 & -0.215595123 \\
\hline 19 & 0 & 0.000974029 & -0.235318131 & -0.216471788 \\
\hline 19.1 & 0 & 0.000970878 & -0.236201953 & -0.217346271 \\
\hline 19.2 & 0 & 0.000967836 & -0.237083605 & -0.218218584 \\
\hline 19.3 & 0 & 0.000964903 & -0.237963097 & -0.21908874 \\
\hline 19.4 & 0 & 0.000962079 & -0.238840443 & -0.219956749 \\
\hline 19.5 & 0 & 0.000959362 & -0.239715654 & -0.220822625 \\
\hline 19.6 & 0 & 0.000956752 & -0.240588741 & -0.221686379 \\
\hline 19.7 & 0 & 0.000954248 & -0.241459717 & -0.222548022 \\
\hline 19.8 & 0 & 0.000951849 & -0.242328593 & -0.223407567 \\
\hline 19.9 & 0 & 0.000949556 & -0.243195381 & -0.224265024 \\
\hline 20 & 0 & 0.000947367 & -0.244060092 & -0.225120406 \\
\hline
\end{tabular}


The Gibbs free energy difference of the four polytypes of boron nitride ( $\mathrm{r}-\mathrm{BN}, \mathrm{h}-\mathrm{BN}, \mathrm{c}-\mathrm{BN}$, and w-BN) as a function of pressure ranging from 0 to $20 \mathrm{GPa}$, calculated with respect to $\mathrm{r}-\mathrm{BN}$ as a reference at a given temperature of 200 Kelvin. The unit of the Gibbs free energy difference for the four polytypes of boron nitride is given in $\mathrm{eV} /$ atom.

\begin{tabular}{lllll}
$\mathbf{P}(\mathbf{G P a})$ & $\mathbf{r}-\mathbf{B N}$ & $\mathbf{h}-\mathbf{B N}$ & $\mathbf{c}-\mathbf{B N}$ & $\mathbf{w}-\mathbf{B N}$ \\
0 & 0 & 0.006413679 & 0.003500911 & 0.020519885 \\
0.1 & 0 & 0.006322303 & 0.001516052 & 0.018544543 \\
0.2 & 0 & 0.006233102 & -0.000448885 & 0.016589122 \\
0.3 & 0 & 0.006145961 & -0.002394641 & 0.014652881 \\
0.4 & 0 & 0.006060774 & -0.004321903 & 0.012735134 \\
0.5 & 0 & 0.005977447 & -0.006231307 & 0.010835245 \\
0.6 & 0 & 0.005895891 & -0.008123443 & 0.008952623 \\
0.7 & 0 & 0.005816029 & -0.009998863 & 0.007086716 \\
0.8 & 0 & 0.005737786 & -0.011858082 & 0.005237009 \\
0.9 & 0 & 0.005661096 & -0.013701586 & 0.003403018 \\
1 & 0 & 0.005585895 & -0.015529828 & 0.001584288 \\
1.1 & 0 & 0.005512127 & -0.017343235 & -0.000219608 \\
1.2 & 0 & 0.005439737 & -0.019142213 & -0.002009075 \\
1.3 & 0 & 0.005368675 & -0.020927142 & -0.003784494 \\
1.4 & 0 & 0.005298895 & -0.022698386 & -0.005546228 \\
1.5 & 0 & 0.005230353 & -0.024456286 & -0.00729462 \\
1.6 & 0 & 0.005163008 & -0.02620117 & -0.009029995 \\
1.7 & 0 & 0.00509682 & -0.027933347 & -0.010752664 \\
1.8 & 0 & 0.005031754 & -0.029653113 & -0.012462923 \\
1.9 & 0 & 0.004967774 & -0.03136075 & -0.014161053 \\
2 & 0 & 0.00490485 & -0.033056527 & -0.015847324 \\
2.1 & 0 & 0.004842949 & -0.034740703 & -0.017521995 \\
2.2 & 0 & 0.004782044 & -0.036413523 & -0.01918531 \\
2.3 & 0 & 0.004722105 & -0.038075225 & -0.020837507 \\
2.4 & 0 & 0.004663107 & -0.039726034 & -0.022478813 \\
2.5 & 0 & 0.004605025 & -0.041366169 & -0.024109445 \\
\hline & 0 & 0.004547836 & -0.042995839 & -0.025729613
\end{tabular}




\begin{tabular}{|c|c|c|c|c|}
\hline 2.7 & 0 & 0.004491515 & -0.044615246 & -0.027339517 \\
\hline 2.8 & 0 & 0.004436043 & -0.046224582 & -0.028939352 \\
\hline 2.9 & 0 & 0.004381398 & -0.047824035 & -0.030529305 \\
\hline 3 & 0 & 0.00432756 & -0.049413785 & -0.032109555 \\
\hline 3.1 & 0 & 0.00427451 & -0.050994005 & -0.033680276 \\
\hline 3.2 & 0 & 0.004222231 & -0.052564864 & -0.035241636 \\
\hline 3.3 & 0 & 0.004170704 & -0.054126523 & -0.036793797 \\
\hline 3.4 & 0 & 0.004119914 & -0.055679139 & -0.038336916 \\
\hline 3.5 & 0 & 0.004069843 & -0.057222863 & -0.039871143 \\
\hline 3.6 & 0 & 0.004020477 & -0.058757842 & -0.041396627 \\
\hline 3.7 & 0 & 0.003971801 & -0.060284219 & -0.042913508 \\
\hline 3.8 & 0 & 0.003923801 & -0.06180213 & -0.044421926 \\
\hline 3.9 & 0 & 0.003876462 & -0.06331171 & -0.045922012 \\
\hline 4 & 0 & 0.003829772 & -0.064813088 & -0.047413897 \\
\hline 4.1 & 0 & 0.003783717 & -0.06630639 & -0.048897707 \\
\hline 4.2 & 0 & 0.003738286 & -0.067791738 & -0.050373563 \\
\hline 4.3 & 0 & 0.003693467 & -0.06926925 & -0.051841584 \\
\hline 4.4 & 0 & 0.003649247 & -0.070739041 & -0.053301885 \\
\hline 4.5 & 0 & 0.003605616 & -0.072201224 & -0.054754579 \\
\hline 4.6 & 0 & 0.003562563 & -0.073655908 & -0.056199774 \\
\hline 4.7 & 0 & 0.003520077 & -0.075103198 & -0.057637576 \\
\hline 4.8 & 0 & 0.00347815 & -0.076543199 & -0.05906809 \\
\hline 4.9 & 0 & 0.003436769 & -0.077976009 & -0.060491414 \\
\hline 5 & 0 & 0.003395927 & -0.079401729 & -0.061907648 \\
\hline 5.1 & 0 & 0.003355615 & -0.080820452 & -0.063316886 \\
\hline 5.2 & 0 & 0.003315822 & -0.082232272 & -0.064719222 \\
\hline 5.3 & 0 & 0.003276541 & -0.08363728 & -0.066114747 \\
\hline 5.4 & 0 & 0.003237763 & -0.085035565 & -0.067503549 \\
\hline 5.5 & 0 & 0.00319948 & -0.086427212 & -0.068885715 \\
\hline 5.6 & 0 & 0.003161684 & -0.087812307 & -0.070261328 \\
\hline 5.7 & 0 & 0.003124367 & -0.089190931 & -0.071630472 \\
\hline 5.8 & 0 & 0.003087522 & -0.090563165 & -0.072993227 \\
\hline
\end{tabular}




\begin{tabular}{|c|c|c|c|c|}
\hline 5.9 & 0 & 0.003051142 & -0.091929088 & -0.074349672 \\
\hline 6 & 0 & 0.00301522 & -0.093288776 & -0.075699882 \\
\hline 6.1 & 0 & 0.002979747 & -0.094642306 & -0.077043935 \\
\hline 6.2 & 0 & 0.002944719 & -0.095989749 & -0.078381902 \\
\hline 6.3 & 0 & 0.002910129 & -0.097331179 & -0.079713856 \\
\hline 6.4 & 0 & 0.002875969 & -0.098666665 & -0.081039868 \\
\hline 6.5 & 0 & 0.002842234 & -0.099996276 & -0.082360006 \\
\hline 6.6 & 0 & 0.002808917 & -0.10132008 & -0.083674337 \\
\hline 6.7 & 0 & 0.002776014 & -0.102638142 & -0.084982927 \\
\hline 6.8 & 0 & 0.002743518 & -0.103950527 & -0.086285841 \\
\hline 6.9 & 0 & 0.002711423 & -0.105257299 & -0.087583143 \\
\hline 7 & 0 & 0.002679724 & -0.106558519 & -0.088874894 \\
\hline 7.1 & 0 & 0.002648415 & -0.107854249 & -0.090161155 \\
\hline 7.2 & 0 & 0.002617493 & -0.109144546 & -0.091441985 \\
\hline 7.3 & 0 & 0.00258695 & -0.110429471 & -0.092717443 \\
\hline 7.4 & 0 & 0.002556783 & -0.111709081 & -0.093987586 \\
\hline 7.5 & 0 & 0.002526986 & -0.11298343 & -0.095252471 \\
\hline 7.6 & 0 & 0.002497555 & -0.114252575 & -0.096512152 \\
\hline 7.7 & 0 & 0.002468484 & -0.11551657 & -0.097766684 \\
\hline 7.8 & 0 & 0.002439771 & -0.116775467 & -0.099016118 \\
\hline 7.9 & 0 & 0.002411409 & -0.118029319 & -0.100260509 \\
\hline 8 & 0 & 0.002383395 & -0.119278177 & -0.101499906 \\
\hline 8.1 & 0 & 0.002355724 & -0.12052209 & -0.10273436 \\
\hline 8.2 & 0 & 0.002328392 & -0.121761109 & -0.10396392 \\
\hline 8.3 & 0 & 0.002301396 & -0.122995281 & -0.105188634 \\
\hline 8.4 & 0 & 0.00227473 & -0.124224654 & -0.10640855 \\
\hline 8.5 & 0 & 0.002248392 & -0.125449274 & -0.107623715 \\
\hline 8.6 & 0 & 0.002222377 & -0.126669189 & -0.108834174 \\
\hline 8.7 & 0 & 0.002196682 & -0.127884442 & -0.110039973 \\
\hline 8.8 & 0 & 0.002171303 & -0.129095078 & -0.111241157 \\
\hline 8.9 & 0 & 0.002146235 & -0.130301141 & -0.112437767 \\
\hline 9 & 0 & 0.002121477 & -0.131502674 & -0.113629848 \\
\hline
\end{tabular}




\begin{tabular}{|c|c|c|c|c|}
\hline .1 & 0 & 0.002097024 & -0.132699718 & -0.114817442 \\
\hline 9.2 & 0 & 0.002072873 & -0.133892315 & -0.11600059 \\
\hline 9.3 & 0 & 0.00204902 & -0.135080506 & -0.117179333 \\
\hline 9.4 & & 0.002025462 & -0.136264331 & -0.11835371 \\
\hline 9.5 & 0 & 0.002002197 & -0.137443829 & -0.119523762 \\
\hline 9.6 & 0 & 0.00197922 & -0.13861904 & -0.120689528 \\
\hline 9.7 & 0 & 0.001956529 & -0.139790002 & -0.121851045 \\
\hline 9.8 & 0 & 0.001934121 & -0.140956751 & -0.123008351 \\
\hline 9.9 & 0 & 0.001911993 & -0.142119326 & -0.124161483 \\
\hline 10 & 0 & 0.001890141 & -0.143277763 & -0.125310478 \\
\hline 10.1 & 0 & 0.001868564 & -0.144432097 & -0.126455371 \\
\hline 10.2 & 0 & 0.001847257 & -0.145582364 & -0.127596199 \\
\hline 10.3 & 0 & 0.001826219 & -0.146728598 & -0.128732995 \\
\hline 10.4 & 0 & 0.001805446 & -0.147870835 & -0.129865794 \\
\hline 10.5 & 0 & 0.001784937 & -0.149009109 & -0.130994631 \\
\hline 10.6 & 0 & 0.001764687 & -0.150143451 & -0.132119537 \\
\hline 10.7 & 0 & 0.001744696 & -0.151273895 & -0.133240547 \\
\hline 10.8 & 0 & 0.001724959 & -0.152400473 & -0.134357692 \\
\hline 10.9 & 0 & 0.001705475 & -0.153523218 & -0.135471005 \\
\hline 11 & 0 & 0.001686241 & -0.154642161 & -0.136580516 \\
\hline 11.1 & 0 & 0.001667255 & -0.155757332 & -0.137686256 \\
\hline 11.2 & 0 & 0.001648515 & -0.156868762 & -0.138788257 \\
\hline 11.3 & 0 & 0.001630018 & -0.157976482 & -0.139886549 \\
\hline 11.4 & 0 & 0.001611761 & -0.159080521 & -0.14098116 \\
\hline 11.5 & 0 & 0.001593743 & -0.160180907 & -0.142072121 \\
\hline 11.6 & 0 & 0.001575961 & -0.161277672 & -0.143159459 \\
\hline 11.7 & 0 & 0.001558413 & -0.162370841 & -0.144243205 \\
\hline 11.8 & 0 & 0.001541097 & -0.163460445 & -0.145323385 \\
\hline 11.9 & 0 & 0.001524011 & -0.164546509 & -0.146400028 \\
\hline 12 & 0 & 0.001507152 & -0.165629063 & -0.14747316 \\
\hline 12.1 & 0 & 0.001490519 & -0.166708132 & -0.14854281 \\
\hline 12.2 & 0 & 0.00147411 & -0.167783744 & -0.149609002 \\
\hline
\end{tabular}




\begin{tabular}{|c|c|c|c|c|}
\hline 12.3 & 0 & 0.001457922 & -0.168855924 & -0.150671765 \\
\hline 12.4 & 0 & 0.001441954 & -0.169924699 & -0.151731123 \\
\hline 12.5 & 0 & 0.001426204 & -0.170990094 & -0.152787103 \\
\hline 12.6 & 0 & 0.001410669 & -0.172052134 & -0.153839728 \\
\hline 12.7 & 0 & 0.001395348 & -0.173110845 & -0.154889026 \\
\hline 12.8 & 0 & 0.00138024 & -0.174166251 & -0.155935019 \\
\hline 12.9 & 0 & 0.001365341 & -0.175218377 & -0.156977733 \\
\hline 13 & 0 & 0.001350651 & -0.176267245 & -0.158017192 \\
\hline 13.1 & 0 & 0.001336168 & -0.177312881 & -0.159053418 \\
\hline 13.2 & 0 & 0.00132189 & -0.178355307 & -0.160086437 \\
\hline 13.3 & 0 & 0.001307815 & -0.179394547 & -0.161116269 \\
\hline 13.4 & 0 & 0.001293941 & -0.180430623 & -0.16214294 \\
\hline 13.5 & 0 & 0.001280267 & -0.181463558 & -0.16316647 \\
\hline 13.6 & 0 & 0.001266791 & -0.182493375 & -0.164186883 \\
\hline 13.7 & 0 & 0.001253512 & -0.183520095 & -0.165204201 \\
\hline 13.8 & 0 & 0.001240428 & -0.18454374 & -0.166218445 \\
\hline 13.9 & 0 & 0.001227537 & -0.185564331 & -0.167229636 \\
\hline 14 & 0 & 0.001214838 & -0.186581891 & -0.168237797 \\
\hline 14.1 & 0 & 0.001202329 & -0.187596439 & -0.169242947 \\
\hline 14.2 & 0 & 0.001190009 & -0.188607997 & -0.170245109 \\
\hline 14.3 & 0 & 0.001177876 & -0.189616586 & -0.171244301 \\
\hline 14.4 & 0 & 0.00116593 & -0.190622224 & -0.17224054 \\
\hline 14.5 & 0 & 0.001154167 & -0.191624933 & -0.173233861 \\
\hline 14.6 & 0 & 0.001142587 & -0.192624732 & -0.174224268 \\
\hline 14.7 & 0 & 0.001131189 & -0.193621641 & -0.175211786 \\
\hline 14.8 & 0 & 0.00111997 & -0.19461568 & -0.176196435 \\
\hline 14.9 & 0 & 0.001108931 & -0.195606866 & -0.177178233 \\
\hline 15 & 0 & 0.001098068 & -0.196595221 & -0.178157199 \\
\hline 15.1 & 0 & 0.001087381 & -0.197580761 & -0.179133353 \\
\hline 15.2 & 0 & 0.00107687 & -0.198563505 & -0.180106712 \\
\hline 15.3 & 0 & 0.001066531 & -0.199543473 & -0.181077296 \\
\hline 15.4 & 0 & 0.001056365 & -0.200520681 & -0.182045121 \\
\hline
\end{tabular}




\begin{tabular}{|c|c|c|c|c|}
\hline 15.5 & 0 & 0.001046369 & -0.201495148 & -0.183010207 \\
\hline 15.6 & 0 & 0.001036543 & -0.202466892 & -0.18397257 \\
\hline 15.7 & 0 & 0.001026884 & -0.20343593 & -0.184932229 \\
\hline 15.8 & 0 & 0.001017393 & -0.20440228 & -0.1858892 \\
\hline 15.9 & 0 & 0.001008068 & -0.205365958 & -0.186843502 \\
\hline 16 & 0 & 0.000998907 & -0.206326982 & -0.18779515 \\
\hline 16.1 & 0 & 0.00098991 & -0.207285368 & -0.188744161 \\
\hline 16.2 & 0 & 0.000981074 & -0.208241133 & -0.189690553 \\
\hline 16.3 & 0 & 0.0009724 & -0.209194293 & -0.190634341 \\
\hline 16.4 & 0 & 0.000963886 & -0.210144865 & -0.191575543 \\
\hline 16.5 & 0 & 0.00095553 & -0.211092866 & -0.192514173 \\
\hline 16.6 & 0 & 0.000947332 & -0.212038309 & -0.193450248 \\
\hline 16.7 & 0 & 0.000939291 & -0.212981213 & -0.194383784 \\
\hline 16.8 & 0 & 0.000931405 & -0.213921591 & -0.195314797 \\
\hline 16.9 & 0 & 0.000923673 & -0.21485946 & -0.196243301 \\
\hline 17 & 0 & 0.000916094 & -0.215794835 & -0.197169312 \\
\hline 17.1 & 0 & 0.000908668 & -0.216727731 & -0.198092846 \\
\hline 17.2 & 0 & 0.000901393 & -0.217658163 & -0.199013917 \\
\hline 17.3 & 0 & 0.000894269 & -0.218586146 & -0.19993254 \\
\hline 17.4 & 0 & 0.000887293 & -0.219511695 & -0.20084873 \\
\hline 17.5 & 0 & 0.000880465 & -0.220434823 & -0.201762501 \\
\hline 17.6 & 0 & 0.000873785 & -0.221355546 & -0.202673867 \\
\hline 17.7 & 0 & 0.000867251 & -0.222273878 & -0.203582844 \\
\hline 17.8 & 0 & 0.000860862 & -0.223189833 & -0.204489445 \\
\hline 17.9 & 0 & 0.000854617 & -0.224103424 & -0.205393684 \\
\hline 18 & 0 & 0.000848515 & -0.225014666 & -0.206295575 \\
\hline 18.1 & 0 & 0.000842556 & -0.225923573 & -0.207195132 \\
\hline 18.2 & 0 & 0.000836738 & -0.226830158 & -0.208092368 \\
\hline 18.3 & 0 & 0.00083106 & -0.227734434 & -0.208987297 \\
\hline 18.4 & 0 & 0.000825522 & -0.228636415 & -0.209879932 \\
\hline 18.5 & 0 & 0.000820123 & -0.229536115 & -0.210770286 \\
\hline 18.6 & 0 & 0.000814861 & -0.230433545 & -0.211658373 \\
\hline
\end{tabular}




$\begin{array}{lllll}18.7 & 0 & 0.000809737 & -0.231328719 & -0.212544205 \\ 18.8 & 0 & 0.000804748 & -0.232221651 & -0.213427795 \\ 18.9 & 0 & 0.000799894 & -0.233112351 & -0.214309156 \\ 19 & 0 & 0.000795175 & -0.234000835 & -0.2151883 \\ 19.1 & 0 & 0.000790589 & -0.234887112 & -0.216065241 \\ 19.2 & 0 & 0.000786136 & -0.235771197 & -0.21693999 \\ 19.3 & 0 & 0.000781814 & -0.236653102 & -0.217812559 \\ 19.4 & 0 & 0.000777623 & -0.237532837 & -0.218682961 \\ 19.5 & 0 & 0.000773563 & -0.238410416 & -0.219551208 \\ 19.6 & 0 & 0.000769632 & -0.239285851 & -0.220417312 \\ 19.7 & 0 & 0.000765829 & -0.240159153 & -0.221281285 \\ 19.8 & 0 & 0.000762154 & -0.241030334 & -0.222143137 \\ 19.9 & 0 & 0.000758606 & -0.241899405 & -0.223002882 \\ 20 & 0 & 0.000755185 & -0.242766379 & -0.22386053\end{array}$

The Gibbs free energy difference of the four polytypes of boron nitride ( $\mathrm{r}-\mathrm{BN}, \mathrm{h}-\mathrm{BN}, \mathrm{c}-\mathrm{BN}$, and w-BN) as a function of pressure ranging from 0 to $20 \mathrm{GPa}$, calculated with respect to $\mathrm{r}-\mathrm{BN}$ as a reference at a given temperature of 300 Kelvin. The unit of the Gibbs free energy difference for the four polytypes of boron nitride is given in $\mathrm{eV} /$ atom.

$\begin{array}{lllll}\mathbf{P}(\mathbf{G P a}) & \mathbf{r}-\mathbf{B N} & \mathbf{h}-\mathbf{B N} & \mathbf{c}-\mathbf{B N} & \mathbf{w}-\mathbf{B N} \\ 0 & 0 & 0.007301054 & 0.007478085 & 0.024424983 \\ 0.1 & 0 & 0.007197565 & 0.005478515 & 0.022434922 \\ 0.2 & 0 & 0.007096304 & 0.003499037 & 0.02046495 \\ 0.3 & 0 & 0.006997164 & 0.001538907 & 0.018514327 \\ 0.4 & 0 & 0.006900047 & -0.000402563 & 0.016582364 \\ 0.5 & 0 & 0.006804863 & -0.00232601 & 0.014668423 \\ 0.6 & 0 & 0.006711529 & -0.004232026 & 0.012771911 \\ 0.7 & 0 & 0.006619969 & -0.006121167 & 0.010892276 \\ 0.8 & 0 & 0.006530114 & -0.007993949 & 0.009028998 \\ 0.9 & 0 & 0.006441898 & -0.009850859 & 0.007181591 \\ 1 & 0 & 0.00635526 & -0.011692354 & 0.005349599 \\ 1.1 & 0 & 0.006270146 & -0.013518865 & 0.003532592\end{array}$




\begin{tabular}{|c|c|c|c|c|}
\hline 1.2 & 0 & 0.006186501 & -0.015330797 & 0.001730161 \\
\hline 1.3 & 0 & 0.006104276 & -0.017128536 & -0.0000580758 \\
\hline 1.4 & 0 & 0.006023426 & -0.018912445 & -0.001832484 \\
\hline 1.5 & 0 & 0.005943906 & -0.02068287 & -0.003593409 \\
\hline 1.6 & 0 & 0.005865676 & -0.02244014 & -0.005341179 \\
\hline 1.7 & 0 & 0.005788697 & -0.024184568 & -0.007076107 \\
\hline 1.8 & 0 & 0.005712933 & -0.02591645 & -0.008798491 \\
\hline 1.9 & 0 & 0.005638348 & -0.027636072 & -0.010508615 \\
\hline 2 & 0 & 0.00556491 & -0.029343706 & -0.012206751 \\
\hline 2.1 & 0 & 0.005492589 & -0.03103961 & -0.013893159 \\
\hline 2.2 & 0 & 0.005421353 & -0.032724034 & -0.015568087 \\
\hline 2.3 & 0 & 0.005351176 & -0.034397217 & -0.017231774 \\
\hline 2.4 & 0 & 0.00528203 & -0.036059386 & -0.018884449 \\
\hline 2.5 & 0 & 0.00521389 & -0.037710763 & -0.020526331 \\
\hline 2.6 & 0 & 0.005146731 & -0.039351558 & -0.022157632 \\
\hline 2.7 & 0 & 0.00508053 & -0.040981974 & -0.023778555 \\
\hline 2.8 & 0 & 0.005015264 & -0.042602207 & -0.025389296 \\
\hline 2.9 & 0 & 0.004950912 & -0.044212445 & -0.026990042 \\
\hline 3 & 0 & 0.004887454 & -0.045812871 & -0.028580977 \\
\hline 3.1 & 0 & 0.004824869 & -0.047403659 & -0.030162274 \\
\hline 3.2 & 0 & 0.004763139 & -0.048984979 & -0.031734105 \\
\hline 3.3 & 0 & 0.004702246 & -0.050556995 & -0.033296632 \\
\hline 3.4 & 0 & 0.004642172 & -0.052119865 & -0.034850014 \\
\hline 3.5 & 0 & 0.0045829 & -0.053673743 & -0.036394403 \\
\hline 3.6 & 0 & 0.004524414 & -0.055218775 & -0.037929949 \\
\hline 3.7 & 0 & 0.004466699 & -0.056755107 & -0.039456794 \\
\hline 3.8 & 0 & 0.004409739 & -0.058282876 & -0.040975077 \\
\hline 3.9 & 0 & 0.00435352 & -0.059802219 & -0.042484935 \\
\hline 4 & 0 & 0.004298027 & -0.061313265 & -0.043986497 \\
\hline 4.1 & 0 & 0.004243248 & -0.062816142 & -0.045479891 \\
\hline 4.2 & 0 & 0.004189168 & -0.064310974 & -0.046965239 \\
\hline 4.3 & 0 & 0.004135777 & -0.06579788 & -0.048442663 \\
\hline
\end{tabular}




\begin{tabular}{|c|c|c|c|c|}
\hline 4.4 & 0 & 0.00408306 & -0.067276976 & -0.049912278 \\
\hline 4.5 & 0 & 0.004031006 & -0.068748376 & -0.051374197 \\
\hline 4.6 & 0 & 0.003979604 & -0.070212191 & -0.052828532 \\
\hline & 0 & 0.003928843 & -0.071668526 & -0.054275388 \\
\hline 4.8 & 0 & 0.003878712 & -0.073117488 & -0.055714871 \\
\hline 4.9 & 0 & 0.003829199 & -0.074559177 & -0.057147082 \\
\hline 5 & 0 & 0.003780296 & -0.075993692 & -0.05857212 \\
\hline 5.1 & 0 & 0.003731992 & -0.07742113 & -0.059990082 \\
\hline 5.2 & 0 & 0.003684277 & -0.078841586 & -0.061401062 \\
\hline 5.3 & 0 & 0.003637143 & -0.08025515 & -0.062805152 \\
\hline 5.4 & 0 & 0.00359058 & -0.081661914 & -0.064202441 \\
\hline 55 & 0 & 0.003544579 & -0.083061963 & -0.065593017 \\
\hline 5.6 & 0 & 0.003499132 & -0.084455383 & -0.066976965 \\
\hline 5.7 & 0 & 0.00345423 & -0.085842258 & -0.068354368 \\
\hline 5.8 & 0 & 0.003409866 & -0.087222669 & -0.069725308 \\
\hline 5.9 & 0 & 0.00336603 & -0.088596696 & -0.071089865 \\
\hline 6 & 0 & 0.003322717 & -0.089964416 & -0.072448116 \\
\hline 6.1 & 0 & 0.003279918 & -0.091325905 & -0.073800136 \\
\hline 6.2 & 0 & 0.003237625 & -0.092681238 & -0.075146001 \\
\hline 6.3 & 0 & 0.003195832 & -0.094030487 & -0.076485784 \\
\hline 6.4 & 0 & 0.003154532 & -0.095373724 & -0.077819554 \\
\hline 6.5 & 0 & 0.003113718 & -0.096711018 & -0.079147383 \\
\hline 6.6 & 0 & 0.003073383 & -0.098042437 & -0.080469337 \\
\hline 6.7 & 0 & 0.003033521 & -0.099368048 & -0.081785485 \\
\hline 6.8 & 0 & 0.002994126 & -0.100687916 & -0.08309589 \\
\hline 6.9 & 0 & 0.00295519 & -0.102002106 & -0.084400618 \\
\hline 7 & 0 & 0.002916709 & -0.103310679 & -0.08569973 \\
\hline 7.1 & 0 & 0.002878677 & -0.104613698 & -0.086993289 \\
\hline 7.2 & 0 & 0.002841088 & -0.105911223 & -0.088281354 \\
\hline 7.3 & 0 & 0.002803935 & -0.107203313 & -0.089563985 \\
\hline 7.4 & 0 & 0.002767214 & -0.108490025 & -0.09084124 \\
\hline 7.5 & 0 & 0.002730919 & -0.109771417 & -0.092113175 \\
\hline
\end{tabular}




\begin{tabular}{|c|c|c|c|c|}
\hline 7.6 & 0 & 0.002695045 & -0.111047544 & -0.093379846 \\
\hline 7.7 & 0 & 0.002659587 & -0.11231846 & -0.094641307 \\
\hline 7.8 & 0 & 0.002624539 & -0.113584221 & -0.095897613 \\
\hline 7.9 & 0 & 0.002589898 & -0.114844877 & -0.097148816 \\
\hline 8 & 0 & 0.002555657 & -0.116100482 & -0.098394968 \\
\hline 8.1 & 0 & 0.002521813 & -0.117351085 & -0.099636119 \\
\hline 8.2 & 0 & 0.00248836 & -0.118596736 & -0.10087232 \\
\hline 8.3 & 0 & 0.002455294 & -0.119837485 & -0.102103619 \\
\hline 8.4 & 0 & 0.002422611 & -0.12107338 & -0.103330064 \\
\hline 8.5 & 0 & 0.002390306 & -0.122304467 & -0.104551704 \\
\hline 8.6 & 0 & 0.002358375 & -0.123530793 & -0.105768583 \\
\hline 8.7 & 0 & 0.002326814 & -0.124752405 & -0.106980748 \\
\hline 8.8 & 0 & 0.002295619 & -0.125969346 & -0.108188243 \\
\hline 8.9 & 0 & 0.002264785 & -0.127181661 & -0.109391114 \\
\hline 9 & 0 & 0.002234309 & -0.128389393 & -0.110589402 \\
\hline 9.1 & 0 & 0.002204187 & -0.129592585 & -0.111783151 \\
\hline 9.2 & 0 & 0.002174415 & -0.130791278 & -0.112972403 \\
\hline 9.3 & 0 & 0.002144989 & -0.131985515 & -0.114157199 \\
\hline 9.4 & 0 & 0.002115906 & -0.133175336 & -0.11533758 \\
\hline 9.5 & 0 & 0.002087161 & -0.13436078 & -0.116513584 \\
\hline 9.6 & 0 & 0.002058753 & -0.135541886 & -0.117685253 \\
\hline 9.7 & 0 & 0.002030676 & -0.136718695 & -0.118852624 \\
\hline 9.8 & 0 & 0.002002928 & -0.137891243 & -0.120015736 \\
\hline 9.9 & 0 & 0.001975505 & -0.139059568 & -0.121174626 \\
\hline 10 & 0 & 0.001948404 & -0.140223707 & -0.122329331 \\
\hline 10.1 & 0 & 0.001921622 & -0.141383697 & -0.123479887 \\
\hline 10.2 & 0 & 0.001895156 & -0.142539572 & -0.12462633 \\
\hline 10.3 & 0 & 0.001869002 & -0.143691369 & -0.125768696 \\
\hline 10.4 & 0 & 0.001843157 & -0.144839123 & -0.126907019 \\
\hline 10.5 & 0 & 0.001817619 & -0.145982866 & -0.128041333 \\
\hline 10.6 & 0 & 0.001792384 & -0.147122634 & -0.129171672 \\
\hline 10.7 & 0 & 0.001767449 & -0.148258458 & -0.130298069 \\
\hline
\end{tabular}




\begin{tabular}{|c|c|c|c|c|}
\hline 10.8 & 0 & 0.001742813 & -0.149390373 & -0.131420557 \\
\hline 10.9 & 0 & 0.001718471 & -0.15051841 & -0.132539169 \\
\hline 11 & 0 & 0.001694421 & -0.151642601 & -0.133653935 \\
\hline 11.1 & 0 & 0.00167066 & -0.152762978 & -0.134764888 \\
\hline 11.2 & 0 & 0.001647186 & -0.15387957 & -0.135872058 \\
\hline 11.3 & 0 & 0.001623996 & -0.15499241 & -0.136975476 \\
\hline 11.4 & 0 & 0.001601087 & -0.156101526 & -0.138075171 \\
\hline 11.5 & 0 & 0.001578457 & -0.157206948 & -0.139171174 \\
\hline 11.6 & 0 & 0.001556104 & -0.158308706 & -0.140263513 \\
\hline 11.7 & 0 & 0.001534024 & -0.159406829 & -0.141352218 \\
\hline 11.8 & 0 & 0.001512215 & -0.160501344 & -0.142437317 \\
\hline 11.9 & 0 & 0.001490675 & -0.16159228 & -0.143518838 \\
\hline 12 & 0 & 0.001469402 & -0.162679665 & -0.144596808 \\
\hline 12.1 & 0 & 0.001448393 & -0.163763526 & -0.145671255 \\
\hline 12.2 & 0 & 0.001427646 & -0.16484389 & -0.146742206 \\
\hline 12.3 & 0 & 0.001407159 & -0.165920782 & -0.147809687 \\
\hline 12.4 & 0 & 0.001386929 & -0.166994231 & -0.148873725 \\
\hline 12.5 & 0 & 0.001366955 & -0.168064261 & -0.149934346 \\
\hline 12.6 & 0 & 0.001347233 & -0.169130898 & -0.150991574 \\
\hline 12.7 & 0 & 0.001327763 & -0.170194168 & -0.152045437 \\
\hline 12.8 & 0 & 0.001308541 & -0.171254094 & -0.153095957 \\
\hline 12.9 & 0 & 0.001289566 & -0.172310703 & -0.15414316 \\
\hline 13 & 0 & 0.001270835 & -0.173364018 & -0.155187071 \\
\hline 13.1 & 0 & 0.001252347 & -0.174414063 & -0.156227713 \\
\hline 13.2 & 0 & 0.0012341 & -0.175460862 & -0.157265109 \\
\hline 13.3 & 0 & 0.001216092 & -0.176504438 & -0.158299284 \\
\hline 13.4 & 0 & 0.001198321 & -0.177544814 & -0.159330261 \\
\hline 13.5 & 0 & 0.001180784 & -0.178582014 & -0.160358062 \\
\hline 13.6 & 0 & 0.00116348 & -0.179616059 & -0.161382709 \\
\hline 13.7 & 0 & 0.001146408 & -0.180646973 & -0.162404226 \\
\hline 13.8 & 0 & 0.001129564 & -0.181674777 & -0.163422634 \\
\hline 13.9 & 0 & 0.001112949 & -0.182699492 & -0.164437955 \\
\hline
\end{tabular}




\begin{tabular}{|c|c|c|c|c|}
\hline 14 & 0 & 0.001096559 & -0.183721141 & -0.16545021 \\
\hline 14.1 & 0 & 0.001080393 & -0.184739745 & -0.166459421 \\
\hline 14.2 & 0 & 0.001064449 & -0.185755323 & -0.167465608 \\
\hline 14.3 & 0 & 0.001048725 & -0.186767899 & -0.168468793 \\
\hline 14.4 & 0 & 0.001033221 & -0.187777491 & -0.169468996 \\
\hline 14.5 & 0 & 0.001017934 & -0.188784121 & -0.170466238 \\
\hline 14.6 & 0 & 0.001002862 & -0.189787807 & -0.171460537 \\
\hline 14.7 & 0 & 0.000988004 & -0.190788571 & -0.172451915 \\
\hline 14.8 & 0 & 0.000973358 & -0.191786431 & -0.17344039 \\
\hline 14.9 & 0 & 0.000958923 & -0.192781407 & -0.174425983 \\
\hline 15 & 0 & 0.000944697 & -0.193773519 & -0.175408711 \\
\hline 15.1 & 0 & 0.000930679 & -0.194762784 & -0.176388595 \\
\hline 15.2 & 0 & 0.000916867 & -0.195749222 & -0.177365653 \\
\hline 15.3 & 0 & 0.000903259 & -0.196732852 & -0.178339903 \\
\hline 15.4 & 0 & 0.000889854 & -0.197713691 & -0.179311364 \\
\hline 15.5 & 0 & 0.000876651 & -0.198691758 & -0.180280054 \\
\hline 15.6 & 0 & 0.000863648 & -0.199667071 & -0.181245991 \\
\hline 15.7 & 0 & 0.000850844 & -0.200639648 & -0.182209193 \\
\hline 15.8 & 0 & 0.000838237 & -0.201609505 & -0.183169676 \\
\hline 15.9 & 0 & 0.000825826 & -0.20257666 & -0.184127459 \\
\hline 16 & 0 & 0.000813609 & -0.203541132 & -0.185082559 \\
\hline 16.1 & 0 & 0.000801586 & -0.204502935 & -0.186034992 \\
\hline 16.2 & 0 & 0.000789754 & -0.205462088 & -0.186984776 \\
\hline 16.3 & 0 & 0.000778113 & -0.206418607 & -0.187931927 \\
\hline 16.4 & 0 & 0.00076666 & -0.207372509 & -0.188876461 \\
\hline 16.5 & 0 & 0.000755396 & -0.208323809 & -0.189818396 \\
\hline 16.6 & 0 & 0.000744318 & -0.209272524 & -0.190757746 \\
\hline 16.7 & 0 & 0.000733426 & -0.210218669 & -0.191694528 \\
\hline 16.8 & 0 & 0.000722717 & -0.211162262 & -0.192628758 \\
\hline 16.9 & 0 & 0.000712191 & -0.212103316 & -0.193560452 \\
\hline 17 & 0 & 0.000701847 & -0.213041849 & -0.194489624 \\
\hline 7.1 & 0 & 0.000691683 & -0.213977874 & -0.195416291 \\
\hline
\end{tabular}




\begin{tabular}{|c|c|c|c|c|}
\hline 17.2 & 0 & 0.000681698 & -0.214911408 & -0.196340467 \\
\hline 17.3 & 0 & 0.000671891 & -0.215842464 & -0.197262167 \\
\hline 17.4 & 0 & 0.000662261 & -0.216771059 & -0.198181406 \\
\hline 17.5 & 0 & 0.000652806 & -0.217697207 & -0.1990982 \\
\hline 17.6 & 0 & 0.000643526 & -0.218620921 & -0.200012561 \\
\hline 17.7 & 0 & 0.000634419 & -0.219542218 & -0.200924506 \\
\hline 17.8 & 0 & 0.000625484 & -0.220461111 & -0.201834049 \\
\hline 17.9 & 0 & 0.00061672 & -0.221377614 & -0.202741202 \\
\hline 18 & 0 & 0.000608126 & -0.222291741 & -0.203645981 \\
\hline 18.1 & 0 & 0.000599701 & -0.223203507 & -0.204548399 \\
\hline 18.2 & 0 & 0.000591444 & -0.224112924 & -0.205448471 \\
\hline 18.3 & 0 & 0.000583353 & -0.225020007 & -0.206346209 \\
\hline 18.4 & 0 & 0.000575428 & -0.225924768 & -0.207241627 \\
\hline 18.5 & 0 & 0.000567668 & -0.226827222 & -0.208134739 \\
\hline 18.6 & 0 & 0.000560071 & -0.227727382 & -0.209025557 \\
\hline 18.7 & 0 & 0.000552637 & -0.22862526 & -0.209914096 \\
\hline 18.8 & 0 & 0.000545364 & -0.22952087 & -0.210800367 \\
\hline 18.9 & 0 & 0.000538252 & -0.230414225 & -0.211684384 \\
\hline 19 & 0 & 0.000531299 & -0.231305336 & -0.212566159 \\
\hline 19.1 & 0 & 0.000524505 & -0.232194218 & -0.213445706 \\
\hline 19.2 & 0 & 0.000517869 & -0.233080882 & -0.214323036 \\
\hline 19.3 & 0 & 0.000511389 & -0.233965341 & -0.215198162 \\
\hline 19.4 & 0 & 0.000505064 & -0.234847606 & -0.216071096 \\
\hline 19.5 & 0 & 0.000498895 & -0.235727692 & -0.216941851 \\
\hline 19.6 & 0 & 0.000492879 & -0.236605608 & -0.217810439 \\
\hline 19.7 & 0 & 0.000487017 & -0.237481368 & -0.218676871 \\
\hline 19.8 & 0 & 0.000481306 & -0.238354983 & -0.219541159 \\
\hline 19.9 & 0 & 0.000475746 & -0.239226464 & -0.220403315 \\
\hline 20 & 0 & 0.000470336 & -0.240095825 & -0.221263351 \\
\hline
\end{tabular}


The Gibbs free energy difference of the four polytypes of boron nitride ( $\mathrm{r}-\mathrm{BN}, \mathrm{h}-\mathrm{BN}, \mathrm{c}-\mathrm{BN}$, and w-BN) as a function of pressure ranging from 0 to $20 \mathrm{GPa}$, calculated with respect to $\mathrm{r}-\mathrm{BN}$ as a reference at a given temperature of 400 Kelvin. The unit of the Gibbs free energy difference for the four polytypes of boron nitride is given in $\mathrm{eV} /$ atom.

\begin{tabular}{|c|c|c|c|c|}
\hline $\mathbf{P}(\mathbf{G P a})$ & $r-B N$ & h-BN & c-BN & $\mathbf{w}-\mathbf{B N}$ \\
\hline 0 & 0 & 0.008220049 & 0.01211015 & 0.028966304 \\
\hline 0.1 & 0 & 0.00810431 & 0.01009675 & 0.026962397 \\
\hline 0.2 & 0 & 0.00799079 & 0.008103545 & 0.024978684 \\
\hline 0.3 & 0 & 0.007879394 & 0.006129795 & 0.023014426 \\
\hline 0.4 & 0 & 0.007770038 & 0.00417482 & 0.021068942 \\
\hline 0.5 & 0 & 0.007662642 & 0.002237984 & 0.019141598 \\
\hline 0.6 & 0 & 0.007557133 & 0.000318698 & 0.017231802 \\
\hline 0.7 & 0 & 0.007453442 & -0.001583591 & 0.015339003 \\
\hline 0.8 & 0 & 0.007351506 & -0.003469398 & 0.013462685 \\
\hline 0.9 & 0 & 0.007251265 & -0.00533921 & 0.011602362 \\
\hline 1 & 0 & 0.007152664 & -0.007193482 & 0.009757578 \\
\hline 1.1 & 0 & 0.007055651 & -0.009032645 & 0.007927903 \\
\hline 1.2 & 0 & 0.006960175 & -0.010857106 & 0.006112929 \\
\hline 1.3 & 0 & 0.006866191 & -0.012667248 & 0.004312273 \\
\hline 1.4 & 0 & 0.006773654 & -0.014463438 & 0.00252557 \\
\hline 1.5 & 0 & 0.006682524 & -0.016246021 & 0.000752472 \\
\hline 1.6 & 0 & 0.006592761 & -0.018015327 & -0.001007349 \\
\hline 1.7 & 0 & 0.006504328 & -0.019771669 & -0.002754206 \\
\hline 1.8 & 0 & 0.006417189 & -0.021515345 & -0.004488399 \\
\hline 1.9 & 0 & 0.00633131 & -0.023246643 & -0.006210213 \\
\hline 2 & 0 & 0.006246661 & -0.024965833 & -0.00791992 \\
\hline 2.1 & 0 & 0.006163209 & -0.026673177 & -0.009617783 \\
\hline 2.2 & 0 & 0.006080926 & -0.028368926 & -0.011304049 \\
\hline 2.3 & 0 & 0.005999785 & -0.030053318 & -0.012978961 \\
\hline 2.4 & 0 & 0.005919757 & -0.031726584 & -0.014642747 \\
\hline 2.5 & 0 & 0.005840819 & -0.033388945 & -0.016295628 \\
\hline 2.6 & 0 & 0.005762945 & -0.035040614 & $-0.01793781 \xi$ \\
\hline
\end{tabular}




\begin{tabular}{|c|c|c|c|c|}
\hline 2.7 & 0 & 0.005686112 & -0.036681794 & -0.019569519 \\
\hline 2.8 & 0 & 0.005610297 & -0.038312683 & -0.02119093 \\
\hline 2.9 & 0 & 0.005535479 & -0.03993347 & -0.022802241 \\
\hline 3 & 0 & 0.005461637 & -0.041544339 & -0.024403633 \\
\hline 3.1 & 0 & 0.005388751 & -0.043145466 & -0.025995284 \\
\hline 3.2 & 0 & 0.005316802 & -0.044737022 & -0.027577365 \\
\hline 3.3 & 0 & 0.005245771 & -0.046319172 & -0.02915004 \\
\hline 3.4 & 0 & 0.005175639 & -0.047892075 & -0.030713469 \\
\hline 3.5 & 0 & 0.005106391 & -0.049455886 & -0.032267806 \\
\hline 3.6 & 0 & 0.005038009 & -0.051010753 & -0.033813201 \\
\hline 3.7 & 0 & 0.004970477 & -0.052556823 & -0.035349798 \\
\hline 3.8 & 0 & 0.004903779 & -0.054094234 & -0.036877738 \\
\hline 3.9 & 0 & 0.004837901 & -0.055623124 & -0.038397157 \\
\hline 4 & 0 & 0.004772828 & -0.057143623 & -0.039908187 \\
\hline 4.1 & 0 & 0.004708545 & -0.058655861 & -0.041410955 \\
\hline 4.2 & 0 & 0.00464504 & -0.060159962 & -0.042905587 \\
\hline 4.3 & 0 & 0.004582298 & -0.061656046 & -0.044392204 \\
\hline 4.4 & 0 & 0.004520307 & -0.063144232 & -0.045870922 \\
\hline 4.5 & 0 & 0.004459055 & -0.064624633 & -0.047341857 \\
\hline 4.6 & 0 & 0.004398529 & -0.066097361 & -0.048805119 \\
\hline 4.7 & 0 & 0.004338717 & -0.067562524 & -0.050260817 \\
\hline 4.8 & 0 & 0.004279609 & -0.069020228 & -0.051709056 \\
\hline 4.9 & 0 & 0.004221194 & -0.070470574 & -0.053149939 \\
\hline 5 & 0 & 0.004163459 & -0.071913664 & -0.054583566 \\
\hline 5.1 & 0 & 0.004106396 & -0.073349595 & -0.056010034 \\
\hline 5.2 & 0 & 0.004049993 & -0.074778461 & -0.057429438 \\
\hline 5.3 & 0 & 0.003994241 & -0.076200356 & -0.058841872 \\
\hline 5.4 & 0 & 0.00393913 & -0.077615369 & -0.060247426 \\
\hline 5.5 & 0 & 0.003884651 & -0.07902359 & -0.061646187 \\
\hline 5.6 & 0 & 0.003830795 & -0.080425104 & -0.063038243 \\
\hline 5.7 & 0 & 0.003777553 & -0.081819996 & -0.064423677 \\
\hline 5.8 & 0 & 0.003724915 & -0.083208348 & -0.065802571 \\
\hline
\end{tabular}




\begin{tabular}{|c|c|c|c|c|}
\hline 5.9 & 0 & 0.003672874 & -0.08459024 & -0.067175007 \\
\hline 6 & 0 & 0.003621422 & -0.085965751 & -0.068541062 \\
\hline 6.1 & 0 & 0.003570549 & -0.087334958 & -0.069900814 \\
\hline 6.2 & 0 & 0.00352025 & -0.088697935 & -0.071254337 \\
\hline 6.3 & 0 & 0.003470514 & -0.090054757 & -0.072601705 \\
\hline 6.4 & 0 & 0.003421337 & -0.091405494 & -0.07394299 \\
\hline 6.5 & 0 & 0.003372708 & -0.092750218 & -0.075278262 \\
\hline 6.6 & 0 & 0.003324623 & -0.094088998 & -0.076607591 \\
\hline 6.7 & 0 & 0.003277074 & -0.0954219 & -0.077931042 \\
\hline 6.8 & 0 & 0.003230053 & -0.096748991 & -0.079248684 \\
\hline 6.9 & 0 & 0.003183555 & -0.098070336 & -0.08056058 \\
\hline 7 & 0 & 0.003137572 & -0.099385997 & -0.081866794 \\
\hline 7.1 & 0 & 0.003092099 & -0.100696038 & -0.083167388 \\
\hline 7.2 & 0 & 0.003047128 & -0.102000519 & -0.084462422 \\
\hline 7.3 & 0 & 0.003002655 & -0.1032995 & -0.085751957 \\
\hline 7.4 & 0 & 0.002958673 & -0.104593038 & -0.087036051 \\
\hline 7.5 & 0 & 0.002915176 & -0.105881193 & -0.088314762 \\
\hline 7.6 & 0 & 0.002872158 & -0.10716402 & -0.089588146 \\
\hline 7.7 & 0 & 0.002829614 & -0.108441574 & -0.090856258 \\
\hline 7.8 & 0 & 0.002787538 & -0.10971391 & -0.092119152 \\
\hline 7.9 & 0 & 0.002745925 & -0.11098108 & -0.093376882 \\
\hline 8 & 0 & 0.002704769 & -0.112243138 & -0.0946295 \\
\hline 8.1 & 0 & 0.002664066 & -0.113500134 & -0.095877057 \\
\hline 8.2 & 0 & 0.002623811 & -0.114752119 & -0.097119604 \\
\hline 8.3 & 0 & 0.002583998 & -0.115999143 & -0.098357191 \\
\hline 8.4 & 0 & 0.002544622 & -0.117241254 & -0.099589865 \\
\hline 8.5 & 0 & 0.002505679 & -0.1184785 & -0.100817676 \\
\hline 8.6 & 0 & 0.002467164 & -0.119710927 & -0.102040669 \\
\hline 8.7 & 0 & 0.002429072 & -0.120938583 & -0.10325889 \\
\hline 8.8 & 0 & 0.002391399 & -0.122161512 & -0.104472386 \\
\hline 8.9 & 0 & 0.00235414 & -0.123379759 & -0.105681202 \\
\hline 9 & 0 & 0.002317292 & -0.124593368 & -0.106885379 \\
\hline
\end{tabular}




\begin{tabular}{|c|c|c|c|c|}
\hline .1 & 0 & 0.002280849 & -0.125802383 & -0.108084963 \\
\hline 9.2 & 0 & 0.002244808 & -0.127006844 & -0.109279995 \\
\hline 9.3 & 0 & 0.002209165 & -0.128206795 & -0.110470518 \\
\hline 9.4 & & 0.002173915 & -0.129402276 & -0.111656571 \\
\hline 9.5 & 0 & 0.002139054 & -0.130593329 & -0.112838196 \\
\hline 9.6 & 0 & 0.002104579 & -0.131779991 & -0.114015433 \\
\hline 9.7 & 0 & 0.002070485 & -0.132962303 & -0.11518832 \\
\hline 9.8 & 0 & 0.002036769 & -0.134140303 & -0.116356896 \\
\hline 9.9 & 0 & 0.002003428 & -0.13531403 & -0.117521199 \\
\hline 10 & 0 & 0.001970457 & -0.136483519 & -0.118681266 \\
\hline 10.1 & 0 & 0.001937853 & -0.13764881 & -0.119837134 \\
\hline 10.2 & 0 & 0.001905612 & -0.138809936 & -0.12098884 \\
\hline 10.3 & 0 & 0.001873732 & -0.139966935 & -0.122136419 \\
\hline 10.4 & 0 & 0.001842208 & -0.14111984 & -0.123279906 \\
\hline 10.5 & 0 & 0.001811037 & -0.142268688 & -0.124419336 \\
\hline 10.6 & 0 & 0.001780216 & -0.143413512 & -0.125554743 \\
\hline 10.7 & 0 & 0.001749742 & -0.144554345 & -0.12668616 \\
\hline 10.8 & 0 & 0.001719611 & -0.145691221 & -0.12781362 \\
\hline 10.9 & 0 & 0.00168982 & -0.146824172 & -0.128937157 \\
\hline 11 & 0 & 0.001660367 & -0.14795323 & -0.130056803 \\
\hline 11.1 & 0 & 0.001631247 & -0.149078428 & -0.131172588 \\
\hline 11.2 & 0 & 0.001602459 & -0.150199796 & -0.132284545 \\
\hline 11.3 & 0 & 0.001573999 & -0.151317366 & -0.133392704 \\
\hline 11.4 & 0 & 0.001545865 & -0.152431167 & -0.134497096 \\
\hline 11.5 & 0 & 0.001518053 & -0.15354123 & -0.13559775 \\
\hline 11.6 & 0 & 0.00149056 & -0.154647585 & -0.136694697 \\
\hline 11.7 & 0 & 0.001463384 & -0.15575026 & -0.137787965 \\
\hline 11.8 & 0 & 0.001436522 & -0.156849284 & -0.138877583 \\
\hline 11.9 & 0 & 0.001409972 & -0.157944685 & -0.13996358 \\
\hline 12 & 0 & 0.00138373 & -0.159036492 & -0.141045983 \\
\hline 12.1 & 0 & 0.001357795 & -0.160124733 & -0.142124821 \\
\hline 12.2 & 0 & 0.001332163 & -0.161209433 & -0.14320012 \\
\hline
\end{tabular}




\begin{tabular}{|c|c|c|c|c|}
\hline 2.3 & 0 & 0.001306832 & -0.162290622 & -0.144271907 \\
\hline 12.4 & 0 & 0.001281799 & -0.163368324 & -0.145340209 \\
\hline 12.5 & 0 & 0.001257062 & -0.164442566 & -0.146405053 \\
\hline 12.6 & 0 & 0.001232619 & -0.165513375 & -0.147466463 \\
\hline 12.7 & 0 & 0.001208467 & -0.166580775 & -0.148524466 \\
\hline 12.8 & 0 & 0.001184604 & -0.167644792 & -0.149579087 \\
\hline 12.9 & 0 & 0.001161028 & -0.16870545 & -0.150630351 \\
\hline 13 & 0 & 0.001137735 & -0.169762775 & -0.151678281 \\
\hline 13.1 & 0 & 0.001114724 & -0.170816791 & -0.152722904 \\
\hline 13.2 & 0 & 0.001091993 & -0.171867521 & -0.153764242 \\
\hline 13.3 & 0 & 0.00106954 & -0.17291499 & -0.15480232 \\
\hline 13.4 & 0 & 0.001047361 & -0.17395922 & -0.15583716 \\
\hline 13.5 & 0 & 0.001025456 & -0.175000235 & -0.156868786 \\
\hline 13.6 & 0 & 0.001003822 & -0.176038057 & -0.15789722 \\
\hline 13.7 & 0 & 0.000982456 & -0.17707271 & -0.158922486 \\
\hline 13.8 & 0 & 0.000961357 & -0.178104216 & -0.159944605 \\
\hline 13.9 & 0 & 0.000940523 & -0.179132596 & -0.1609636 \\
\hline 14 & 0 & 0.000919952 & -0.180157872 & -0.161979492 \\
\hline 14.1 & 0 & 0.000899642 & -0.181180066 & -0.162992303 \\
\hline 14.2 & 0 & 0.00087959 & -0.182199199 & -0.164002054 \\
\hline 14.3 & 0 & 0.000859795 & -0.183215292 & -0.165008766 \\
\hline 14.4 & 0 & 0.000840255 & -0.184228365 & -0.166012459 \\
\hline 14.5 & 0 & 0.000820968 & -0.185238441 & -0.167013156 \\
\hline 14.6 & 0 & 0.000801933 & -0.186245537 & -0.168010875 \\
\hline 14.7 & 0 & 0.000783146 & -0.187249676 & -0.169005636 \\
\hline 14.8 & 0 & 0.000764607 & -0.188250876 & -0.16999746 \\
\hline 14.9 & 0 & 0.000746314 & -0.189249157 & -0.170986367 \\
\hline 15 & 0 & 0.000728265 & -0.190244539 & -0.171972375 \\
\hline 15.1 & 0 & 0.000710458 & -0.19123704 & -0.172955503 \\
\hline 15.2 & 0 & 0.000692891 & -0.192226681 & -0.173935772 \\
\hline 15.3 & 0 & 0.000675564 & -0.193213478 & -0.174913199 \\
\hline 15.4 & 0 & 0.000658473 & -0.194197452 & -0.175887803 \\
\hline
\end{tabular}




\begin{tabular}{|c|c|c|c|c|}
\hline 15.5 & 0 & 0.000641617 & -0.19517862 & -0.176859602 \\
\hline 15.6 & 0 & 0.000624996 & -0.196157 & -0.177828615 \\
\hline 15.7 & 0 & 0.000608606 & -0.197132611 & -0.178794859 \\
\hline 15.8 & 0 & 0.000592447 & -0.19810547 & -0.179758353 \\
\hline 15.9 & 0 & 0.000576517 & -0.199075595 & -0.180719113 \\
\hline 16 & 0 & 0.000560814 & -0.200043003 & -0.181677158 \\
\hline 16.1 & 0 & 0.000545337 & -0.201007711 & -0.182632504 \\
\hline 16.2 & 0 & 0.000530084 & -0.201969737 & -0.183585168 \\
\hline 16.3 & 0 & 0.000515054 & -0.202929096 & -0.184535168 \\
\hline 16.4 & 0 & 0.000500245 & -0.203885807 & -0.18548252 \\
\hline 16.5 & 0 & 0.000485656 & -0.204839885 & -0.18642724 \\
\hline 16.6 & 0 & 0.000471285 & -0.205791347 & -0.187369345 \\
\hline 16.7 & 0 & 0.000457131 & -0.206740208 & -0.18830885 \\
\hline 16.8 & 0 & 0.000443192 & -0.207686486 & -0.189245773 \\
\hline 16.9 & 0 & 0.000429467 & -0.208630194 & -0.190180128 \\
\hline 17 & 0 & 0.000415955 & -0.209571351 & -0.191111932 \\
\hline 17.1 & 0 & 0.000402654 & -0.21050997 & -0.192041199 \\
\hline 17.2 & 0 & 0.000389562 & -0.211446067 & -0.192967946 \\
\hline 17.3 & 0 & 0.000376679 & -0.212379657 & -0.193892187 \\
\hline 17.4 & 0 & 0.000364004 & -0.213310756 & -0.194813938 \\
\hline 17.5 & 0 & 0.000351533 & -0.214239379 & -0.195733213 \\
\hline 17.6 & 0 & 0.000339268 & -0.215165539 & -0.196650028 \\
\hline 17.7 & 0 & 0.000327205 & -0.216089252 & -0.197564396 \\
\hline 17.8 & 0 & 0.000315344 & -0.217010532 & -0.198476332 \\
\hline 17.9 & 0 & 0.000303684 & -0.217929394 & -0.199385851 \\
\hline 18 & 0 & 0.000292223 & -0.218845851 & -0.200292967 \\
\hline 18.1 & 0 & 0.00028096 & -0.219759917 & -0.201197693 \\
\hline 18.2 & 0 & 0.000269894 & -0.220671608 & -0.202100044 \\
\hline 18.3 & 0 & 0.000259024 & -0.221580936 & -0.203000034 \\
\hline 18.4 & 0 & 0.000248348 & -0.222487915 & -0.203897675 \\
\hline 18.5 & 0 & 0.000237865 & -0.223392558 & -0.204792983 \\
\hline 18.6 & 0 & 0.000227574 & -0.224294879 & -0.205685969 \\
\hline
\end{tabular}




$\begin{array}{lllll}18.7 & 0 & 0.000217474 & -0.225194892 & -0.206576648 \\ 18.8 & 0 & 0.000207564 & -0.226092609 & -0.207465032 \\ 18.9 & 0 & 0.000197842 & -0.226988043 & -0.208351135 \\ 19 & 0 & 0.000188308 & -0.227881208 & -0.209234969 \\ 19.1 & 0 & 0.00017896 & -0.228772115 & -0.210116547 \\ 19.2 & 0 & 0.000169797 & -0.229660779 & -0.210995883 \\ 19.3 & 0 & 0.000160818 & -0.23054721 & -0.211872987 \\ 19.4 & 0 & 0.000152022 & -0.231431422 & -0.212747874 \\ 19.5 & 0 & 0.000143408 & -0.232313428 & -0.213620554 \\ 19.6 & 0 & 0.000134975 & -0.233193238 & -0.214491041 \\ 19.7 & 0 & 0.000126721 & -0.234070866 & -0.215359347 \\ 19.8 & 0 & 0.000118647 & -0.234946324 & -0.216225483 \\ 19.9 & 0 & 0.00011075 & -0.235819622 & -0.217089461 \\ 20 & 0 & 0.000103029 & -0.236690774 & -0.217951294\end{array}$

The Gibbs free energy difference of the four polytypes of boron nitride (r-BN, h-BN, c-BN, and w-BN) as a function of pressure ranging from 0 to $20 \mathrm{GPa}$, calculated with respect to $\mathrm{r}-\mathrm{BN}$ as a reference at a given temperature of 500 Kelvin. The unit of the Gibbs free energy difference for the four polytypes of boron nitride is given in $\mathrm{eV} /$ atom.

$\begin{array}{lllll}\mathbf{P}(\mathbf{G P a}) & \mathbf{r}-\mathbf{B N} & \mathbf{h}-\mathbf{B N} & \mathbf{c}-\mathbf{B N} & \mathbf{w}-\mathbf{B N} \\ 0 & 0 & 0.009194329 & 0.016953686 & 0.033695495 \\ 0.1 & 0 & 0.009066246 & 0.014927665 & 0.031678983 \\ 0.2 & 0 & 0.00894032 & 0.012921875 & 0.029682702 \\ 0.3 & 0 & 0.008816478 & 0.010935592 & 0.027705927 \\ 0.4 & 0 & 0.008694651 & 0.008968142 & 0.025747985 \\ 0.5 & 0 & 0.008574775 & 0.0070189 & 0.023808251 \\ 0.6 & 0 & 0.008456788 & 0.005087283 & 0.02188614 \\ 0.7 & 0 & 0.008340633 & 0.003172744 & 0.019981108 \\ 0.8 & 0 & 0.008226256 & 0.001274772 & 0.018092642 \\ 0.9 & 0 & 0.008113605 & -0.000607115 & 0.016220261 \\ 1 & 0 & 0.008002632 & -0.00247337 & 0.014363512 \\ 1.1 & 0 & 0.007893289 & -0.00432442 & 0.012521966\end{array}$




\begin{tabular}{|c|c|c|c|c|}
\hline 1.2 & 0 & 0.007785533 & -0.00616067 & 0.01069522 \\
\hline 1.3 & 0 & 0.007679323 & -0.007982504 & 0.008882891 \\
\hline 1.4 & 0 & 0.007574618 & -0.009790283 & 0.007084615 \\
\hline 1.5 & 0 & 0.007471379 & -0.011584355 & 0.005300046 \\
\hline 1.6 & 0 & 0.007369572 & -0.013365047 & 0.003528857 \\
\hline 1.7 & 0 & 0.007269161 & -0.015132672 & 0.001770734 \\
\hline 1.8 & 0 & 0.007170113 & -0.016887529 & 0.0000253781 \\
\hline 1.9 & 0 & 0.007072396 & -0.018629903 & -0.001707495 \\
\hline 2 & 0 & 0.00697598 & -0.020360067 & -0.003428158 \\
\hline 2.1 & 0 & 0.006880835 & -0.022078282 & -0.005136874 \\
\hline 2.2 & 0 & 0.006786935 & -0.023784799 & -0.00683389 \\
\hline 2.3 & 0 & 0.006694251 & -0.025479856 & -0.008519449 \\
\hline 2.4 & 0 & 0.006602758 & -0.027163686 & -0.01019378 \\
\hline 2.5 & 0 & 0.006512431 & -0.028836508 & -0.011857105 \\
\hline 2.6 & 0 & 0.006423247 & -0.030498537 & -0.013509637 \\
\hline 2.7 & 0 & 0.006335182 & -0.032149978 & -0.01515158 \\
\hline 2.8 & 0 & 0.006248213 & -0.033791027 & -0.016783133 \\
\hline 2.9 & 0 & 0.006162321 & -0.035421876 & -0.018404486 \\
\hline 3 & 0 & 0.006077483 & -0.037042708 & -0.020015823 \\
\hline 3.1 & 0 & 0.005993681 & -0.038653701 & -0.021617321 \\
\hline 3.2 & 0 & 0.005910894 & -0.040255025 & -0.023209152 \\
\hline 3.3 & 0 & 0.005829105 & -0.041846848 & -0.024791481 \\
\hline 3.4 & 0 & 0.005748294 & -0.043429328 & -0.026364469 \\
\hline 3.5 & 0 & 0.005668445 & -0.045002622 & -0.02792827 \\
\hline 3.6 & 0 & 0.005589541 & -0.046566879 & -0.029483036 \\
\hline 3.7 & 0 & 0.005511565 & -0.048122246 & -0.031028911 \\
\hline 3.8 & 0 & 0.005434502 & -0.049668862 & -0.032566037 \\
\hline 3.9 & 0 & 0.005358337 & -0.051206865 & -0.03409455 \\
\hline 4 & 0 & 0.005283053 & -0.052736389 & -0.035614584 \\
\hline 4.1 & 0 & 0.005208638 & -0.054257562 & -0.037126268 \\
\hline 4.2 & 0 & 0.005135076 & -0.055770508 & -0.038629727 \\
\hline 4.3 & 0 & 0.005062355 & -0.057275351 & -0.040125082 \\
\hline
\end{tabular}




\begin{tabular}{|c|c|c|c|c|}
\hline & 0 & 0.00499046 & -0.058772209 & -0.041612453 \\
\hline 4.5 & 0 & 0.00491938 & -0.060261196 & -0.043091954 \\
\hline 4.6 & 0 & 0.004849101 & -0.061742425 & -0.044563697 \\
\hline 47 & & 0.004779611 & -0.063216004 & -0.046027792 \\
\hline 4.8 & 0 & 0.004710899 & -0.064682041 & -0.047484345 \\
\hline 4.9 & 0 & 0.004642953 & -0.066140638 & -0.048933458 \\
\hline 5 & 0 & 0.004575762 & -0.067591896 & -0.050375234 \\
\hline 5.1 & 0 & 0.004509314 & -0.069035914 & -0.05180977 \\
\hline 5.2 & 0 & 0.0044436 & -0.070472788 & -0.053237162 \\
\hline 5.3 & 0 & 0.004378608 & -0.071902611 & -0.054657503 \\
\hline 5.4 & 0 & 0.004314329 & -0.073325473 & -0.056070886 \\
\hline 5.5 & 0 & 0.004250753 & -0.074741466 & -0.057477399 \\
\hline 5.6 & 0 & 0.00418787 & -0.076150674 & -0.058877128 \\
\hline 5.7 & 0 & 0.004125671 & -0.077553184 & -0.06027016 \\
\hline 5.8 & 0 & 0.004064146 & -0.078949078 & -0.061656577 \\
\hline 5.9 & 0 & 0.004003287 & -0.080338437 & -0.063036459 \\
\hline 6 & 0 & 0.003943084 & -0.081721341 & -0.064409887 \\
\hline 6.1 & 0 & 0.00388353 & -0.083097867 & -0.065776938 \\
\hline 6.2 & 0 & 0.003824615 & -0.084468091 & -0.067137688 \\
\hline 6.3 & 0 & 0.003766333 & -0.085832088 & -0.06849221 \\
\hline 6.4 & 0 & 0.003708674 & -0.087189928 & -0.069840578 \\
\hline 6.5 & 0 & 0.003651631 & -0.088541685 & -0.071182862 \\
\hline 6.6 & 0 & 0.003595196 & -0.089887427 & -0.072519133 \\
\hline 6.7 & 0 & 0.003539362 & -0.091227223 & -0.073849457 \\
\hline 6.8 & 0 & 0.003484122 & -0.092561138 & -0.075173903 \\
\hline 6.9 & 0 & 0.003429468 & -0.093889239 & -0.076492534 \\
\hline 7 & 0 & 0.003375393 & -0.09521159 & -0.077805417 \\
\hline 7.1 & 0 & 0.003321891 & -0.096528253 & -0.079112611 \\
\hline 7.2 & 0 & 0.003268955 & -0.09783929 & -0.080414181 \\
\hline 7.3 & 0 & 0.003216578 & -0.099144761 & -0.081710186 \\
\hline 7.4 & 0 & 0.003164754 & -0.100444725 & -0.083000685 \\
\hline 7.5 & 0 & 0.003113476 & -0.101739241 & -0.084285735 \\
\hline
\end{tabular}




\begin{tabular}{|c|c|c|c|c|}
\hline 7.6 & 0 & 0.003062738 & -0.103028365 & -0.085565395 \\
\hline 7.7 & 0 & 0.003012535 & -0.104312153 & -0.08683972 \\
\hline 7.8 & 0 & 0.00296286 & -0.10559066 & -0.088108765 \\
\hline 7.9 & 0 & 0.002913707 & -0.10686394 & -0.089372583 \\
\hline 8 & 0 & 0.002865071 & -0.108132045 & -0.090631228 \\
\hline 8.1 & 0 & 0.002816946 & -0.109395028 & -0.091884751 \\
\hline 8.2 & 0 & 0.002769326 & -0.11065294 & -0.093133204 \\
\hline 8.3 & 0 & 0.002722207 & -0.11190583 & -0.094376635 \\
\hline 8.4 & 0 & 0.002675583 & -0.113153748 & -0.095615096 \\
\hline 8.5 & 0 & 0.002629449 & -0.114396742 & -0.096848633 \\
\hline 8.6 & 0 & 0.002583798 & -0.115634859 & -0.098077294 \\
\hline 8.7 & 0 & 0.002538628 & -0.116868147 & -0.099301127 \\
\hline 8.8 & 0 & 0.002493932 & -0.118096651 & -0.100520177 \\
\hline 8.9 & 0 & 0.002449706 & -0.119320416 & -0.101734488 \\
\hline 9 & 0 & 0.002405945 & -0.120539487 & -0.102944106 \\
\hline 9.1 & 0 & 0.002362644 & -0.121753906 & -0.104149074 \\
\hline 9.2 & 0 & 0.0023198 & -0.122963718 & -0.105349435 \\
\hline 9.3 & 0 & 0.002277406 & -0.124168964 & -0.106545231 \\
\hline 9.4 & 0 & 0.002235459 & -0.125369687 & -0.107736504 \\
\hline 9.5 & 0 & 0.002193955 & -0.126565926 & -0.108923295 \\
\hline 9.6 & 0 & 0.002152889 & -0.127757721 & -0.110105643 \\
\hline 9.7 & 0 & 0.002112257 & -0.128945113 & -0.111283589 \\
\hline 9.8 & 0 & 0.002072054 & -0.130128141 & -0.112457171 \\
\hline 9.9 & 0 & 0.002032278 & -0.131306843 & -0.113626427 \\
\hline 10 & 0 & 0.001992923 & -0.132481256 & -0.114791397 \\
\hline 10.1 & 0 & 0.001953986 & -0.133651418 & -0.115952116 \\
\hline 10.2 & 0 & 0.001915463 & -0.134817365 & -0.117108621 \\
\hline 10.3 & 0 & 0.00187735 & -0.135979134 & -0.118260949 \\
\hline 10.4 & 0 & 0.001839644 & -0.13713676 & -0.119409134 \\
\hline 10.5 & 0 & 0.00180234 & -0.138290279 & -0.120553213 \\
\hline 10.6 & 0 & 0.001765435 & -0.139439723 & -0.121693219 \\
\hline 10.7 & 0 & 0.001728926 & -0.140585129 & -0.122829186 \\
\hline
\end{tabular}




\begin{tabular}{|c|c|c|c|c|}
\hline 10.8 & 0 & 0.001692808 & -0.141726528 & -0.123961149 \\
\hline 10.9 & 0 & 0.001657079 & -0.142863955 & -0.12508914 \\
\hline 11 & 0 & 0.001621735 & -0.143997441 & -0.126213191 \\
\hline 11.1 & 0 & 0.001586772 & -0.145127019 & -0.127333335 \\
\hline 11.2 & 0 & 0.001552188 & -0.14625272 & -0.128449603 \\
\hline 1.3 & 0 & 0.001517979 & -0.147374576 & -0.129562027 \\
\hline 11.4 & 0 & 0.001484142 & -0.148492617 & -0.130670637 \\
\hline 11.5 & 0 & 0.001450674 & -0.149606874 & -0.131775463 \\
\hline 11.6 & 0 & 0.001417571 & -0.150717377 & -0.132876536 \\
\hline 11.7 & 0 & 0.00138483 & -0.151824154 & -0.133973886 \\
\hline 11.8 & 0 & 0.001352449 & -0.152927236 & -0.13506754 \\
\hline 11.9 & 0 & 0.001320424 & -0.154026651 & -0.136157528 \\
\hline 12 & 0 & 0.001288753 & -0.155122427 & -0.137243879 \\
\hline 12.1 & 0 & 0.001257433 & -0.156214592 & -0.138326619 \\
\hline 12.2 & 0 & 0.00122646 & -0.157303174 & -0.139405778 \\
\hline 12.3 & 0 & 0.001195832 & -0.158388201 & -0.140481381 \\
\hline 12.4 & 0 & 0.001165546 & -0.159469698 & -0.141553457 \\
\hline 12.5 & 0 & 0.001135599 & -0.160547692 & -0.14262203 \\
\hline 12.6 & 0 & 0.001105989 & -0.161622211 & -0.143687129 \\
\hline 12.7 & 0 & 0.001076713 & -0.162693279 & -0.144748778 \\
\hline 12.8 & 0 & 0.001047768 & -0.163760922 & -0.145807003 \\
\hline 12.9 & 0 & 0.001019152 & -0.164825165 & -0.146861829 \\
\hline 13 & 0 & 0.000990862 & -0.165886033 & -0.147913282 \\
\hline 13.1 & 0 & 0.000962896 & -0.166943551 & -0.148961385 \\
\hline 13.2 & 0 & 0.000935251 & -0.167997743 & -0.150006163 \\
\hline 13.3 & 0 & 0.000907924 & -0.169048633 & -0.15104764 \\
\hline 13.4 & 0 & 0.000880914 & -0.170096245 & -0.15208584 \\
\hline 13.5 & 0 & 0.000854217 & -0.171140601 & -0.153120785 \\
\hline 13.6 & 0 & 0.000827832 & -0.172181727 & -0.154152501 \\
\hline 13.7 & 0 & 0.000801756 & -0.173219642 & -0.155181008 \\
\hline 13.8 & 0 & 0.000775986 & -0.174254372 & -0.156206329 \\
\hline 13.9 & 0 & 0.000750521 & -0.175285937 & -0.157228488 \\
\hline
\end{tabular}




\begin{tabular}{|c|c|c|c|c|}
\hline 14 & 0 & 0.000725359 & -0.17631436 & -0.158247505 \\
\hline 14.1 & 0 & 0.000700496 & -0.177339663 & -0.159263403 \\
\hline 14.2 & 0 & 0.000675931 & -0.178361868 & -0.160276203 \\
\hline 14.3 & 0 & 0.000651662 & -0.179380994 & -0.161285927 \\
\hline 14.4 & 0 & 0.000627686 & -0.180397064 & -0.162292595 \\
\hline 14.5 & 0 & 0.000604002 & -0.181410098 & -0.163296229 \\
\hline 14.6 & 0 & 0.000580607 & -0.182420117 & -0.164296848 \\
\hline 14.7 & 0 & 0.000557499 & -0.183427141 & -0.165294473 \\
\hline 14.8 & 0 & 0.000534676 & -0.18443119 & -0.166289125 \\
\hline 14.9 & 0 & 0.000512137 & -0.185432285 & -0.167280823 \\
\hline 15 & 0 & 0.000489878 & -0.186430443 & -0.168269586 \\
\hline 15.1 & 0 & 0.000467899 & -0.187425687 & -0.169255435 \\
\hline 15.2 & 0 & 0.000446197 & -0.188418033 & -0.170238387 \\
\hline 15.3 & 0 & 0.00042477 & -0.189407501 & -0.171218463 \\
\hline 15.4 & 0 & 0.000403617 & -0.190394111 & -0.172195682 \\
\hline 15.5 & 0 & 0.000382735 & -0.19137788 & -0.17317006 \\
\hline 15.6 & 0 & 0.000362123 & -0.192358827 & -0.174141618 \\
\hline 15.7 & 0 & 0.000341779 & -0.19333697 & -0.175110373 \\
\hline 15.8 & 0 & 0.000321701 & -0.194312327 & -0.176076344 \\
\hline 15.9 & 0 & 0.000301887 & -0.195284916 & -0.177039547 \\
\hline 16 & 0 & 0.000282336 & -0.196254755 & -0.178 \\
\hline 16.1 & 0 & 0.000263045 & -0.19722186 & -0.178957722 \\
\hline 16.2 & 0 & 0.000244013 & -0.19818625 & -0.179912729 \\
\hline 16.3 & 0 & 0.000225239 & -0.199147941 & -0.180865038 \\
\hline 16.4 & 0 & 0.00020672 & -0.20010695 & -0.181814667 \\
\hline 16.5 & 0 & 0.000188455 & -0.201063293 & -0.182761631 \\
\hline 16.6 & 0 & 0.000170442 & -0.202016988 & -0.183705947 \\
\hline 16.7 & 0 & 0.00015268 & -0.202968051 & -0.184647633 \\
\hline 16.8 & 0 & 0.000135167 & -0.203916497 & -0.185586703 \\
\hline 16.9 & 0 & 0.000117902 & -0.204862343 & -0.186523174 \\
\hline 17 & 0 & 0.000100882 & -0.205805605 & -0.187457062 \\
\hline & 0 & 0.000084106 & -0.206746298 & -0.188388382 \\
\hline
\end{tabular}




\begin{tabular}{lllll}
17.2 & 0 & 0.000067573 & -0.207684438 & -0.18931715 \\
17.3 & 0 & 0.0000512811 & -0.208620041 & -0.190243382 \\
17.4 & 0 & 0.0000352289 & -0.209553121 & -0.191167093 \\
17.5 & 0 & 0.0000194148 & -0.210483693 & -0.192088297 \\
17.6 & 0 & 0.0000038373 & -0.211411773 & -0.19300701 \\
17.7 & 0 & -0.000011505 & -0.212337376 & -0.193923246 \\
17.8 & 0 & -0.0000266136 & -0.213260515 & -0.19483702 \\
17.9 & 0 & -0.00004149 & -0.214181206 & -0.195748348 \\
18 & 0 & -0.0000561355 & -0.215099463 & -0.196657242 \\
18.1 & 0 & -0.0000705515 & -0.2160153 & -0.197563717 \\
18.2 & 0 & -0.0000847396 & -0.216928731 & -0.198467787 \\
18.3 & 0 & -0.000098701 & -0.21783977 & -0.199369467 \\
18.4 & 0 & -0.000112437 & -0.218748431 & -0.20026877 \\
18.5 & 0 & -0.000125949 & -0.219654728 & -0.20116571 \\
18.6 & 0 & -0.000139239 & -0.220558674 & -0.2020603 \\
18.7 & 0 & -0.000152307 & -0.221460283 & -0.202952554 \\
18.8 & 0 & -0.000165156 & -0.222359567 & -0.203842484 \\
18.9 & 0 & -0.000177785 & -0.223256541 & -0.204730105 \\
19 & 0 & -0.000190198 & -0.224151216 & -0.20561543 \\
19.1 & 0 & -0.000202394 & -0.225043607 & -0.206498471 \\
19.2 & 0 & -0.000214376 & -0.225933726 & -0.20737924 \\
19.3 & 0 & -0.000226144 & -0.226821586 & -0.208257752 \\
19.4 & 0 & -0.000237699 & -0.227707198 & -0.209134018 \\
19.5 & 0 & -0.000249044 & -0.228590577 & -0.210008051 \\
19.6 & 0 & -0.000260179 & -0.229471733 & -0.210879863 \\
\hline 19.7 & 0 & -0.000271105 & -0.23035068 & -0.211749467 \\
\hline 10 & -0.000281823 & -0.231227429 & -0.212616874 \\
10.000292335 & -0.232101993 & -0.213482097 \\
10.000302642 & -0.232974383 & -0.214345148
\end{tabular}


The Gibbs free energy difference of the four polytypes of boron nitride ( $\mathrm{r}-\mathrm{BN}, \mathrm{h}-\mathrm{BN}, \mathrm{c}-\mathrm{BN}$, and w-BN) as a function of pressure ranging from 0 to $20 \mathrm{GPa}$, calculated with respect to $\mathrm{r}-\mathrm{BN}$ as a reference at a given temperature of 600 Kelvin. The unit of the Gibbs free energy difference for the four polytypes of boron nitride is given in $\mathrm{eV} / \mathrm{atom}$.

\begin{tabular}{lllll}
$\mathbf{P}(\mathbf{G P a}) \mathbf{r}-\mathbf{B N}$ & $\mathbf{h}-\mathbf{B N}$ & $\mathbf{c}-\mathbf{B N}$ & $\mathbf{w}-\mathbf{B N}$ \\
0 & 0 & 0.010242043 & 0.02182836 & 0.03845264 \\
0.1 & 0 & 0.010100947 & 0.019790542 & 0.036424346 \\
0.2 & 0 & 0.009961919 & 0.017772942 & 0.03441627 \\
0.3 & 0 & 0.009824909 & 0.01577485 & 0.032427701 \\
0.4 & 0 & 0.009689869 & 0.013795608 & 0.030457982 \\
0.5 & 0 & 0.00955675 & 0.011834602 & 0.028506499 \\
0.6 & 0 & 0.009425507 & 0.00989126 & 0.026572679 \\
0.7 & 0 & 0.009296094 & 0.007965043 & 0.024655984 \\
0.8 & 0 & 0.009168468 & 0.006055447 & 0.02275591 \\
0.9 & 0 & 0.009042588 & 0.004161997 & 0.020871981 \\
1 & 0 & 0.008918412 & 0.002284246 & 0.019003751 \\
1.1 & 0 & 0.008795902 & 0.00042177 & 0.017150795 \\
1.2 & 0 & 0.00867502 & -0.001425831 & 0.015312714 \\
1.3 & 0 & 0.00855573 & -0.003258936 & 0.013489128 \\
1.4 & 0 & 0.008437997 & -0.005077907 & 0.011679676 \\
1.5 & 0 & 0.008321788 & -0.006883086 & 0.009884015 \\
1.6 & 0 & 0.008207068 & -0.0086748 & 0.008101819 \\
1.7 & 0 & 0.008093809 & -0.01045336 & 0.006332777 \\
1.8 & 0 & 0.007981978 & -0.012219063 & 0.004576591 \\
1.9 & 0 & 0.007871547 & -0.013972193 & 0.002832977 \\
2 & 0 & 0.007762487 & -0.015713023 & 0.001101664 \\
2.1 & 0 & 0.007654772 & -0.017441811 & -0.00061761 \\
2.2 & 0 & 0.007548375 & -0.01915881 & -0.002325093 \\
2.3 & 0 & 0.007443271 & -0.020864256 & -0.004021025 \\
2.4 & 0 & 0.007339434 & -0.022558382 & -0.005705637 \\
2.5 & 0 & 0.007236842 & -0.024241409 & -0.007379149 \\
\hline .6 & 0 & 0.007135471 & -0.025913548 & -0.009041776
\end{tabular}




\begin{tabular}{|c|c|c|c|c|}
\hline 2.7 & 0 & 0.007035299 & -0.027575006 & -0.010693722 \\
\hline 2.8 & 0 & 0.006936304 & -0.029225981 & -0.012335184 \\
\hline 2.9 & 0 & 0.006838466 & -0.030866662 & -0.013966354 \\
\hline 3 & 0 & 0.006741764 & -0.032497234 & -0.015587415 \\
\hline 3.1 & 0 & 0.006646178 & -0.034117875 & -0.017198546 \\
\hline 3.2 & 0 & 0.00655169 & -0.035728756 & -0.018799917 \\
\hline 3.3 & 0 & 0.006458281 & -0.037330044 & -0.020391696 \\
\hline 3.4 & 0 & 0.006365933 & -0.038921899 & -0.021974043 \\
\hline 3.5 & 0 & 0.006274629 & -0.040504477 & -0.023547113 \\
\hline 3.6 & 0 & 0.006184352 & -0.042077929 & -0.025111058 \\
\hline 3.7 & 0 & 0.006095085 & -0.043642401 & -0.026666023 \\
\hline 3.8 & 0 & 0.006006812 & -0.045198035 & -0.02821215 \\
\hline 3.9 & 0 & 0.005919518 & -0.046744967 & -0.029749577 \\
\hline 4 & 0 & 0.005833187 & -0.048283332 & -0.031278437 \\
\hline 4.1 & 0 & 0.005747806 & -0.04981326 & -0.03279886 \\
\hline 4.2 & 0 & 0.005663359 & -0.051334875 & -0.034310972 \\
\hline 4.3 & 0 & 0.005579833 & -0.052848301 & -0.035814895 \\
\hline 4.4 & 0 & 0.005497214 & -0.054353657 & -0.037310748 \\
\hline..+ & 0 & 0.005415489 & -0.055851058 & -0.038798648 \\
\hline 4.6 & 0 & 0.005334645 & -0.057340618 & -0.040278706 \\
\hline 4.7 & 0 & 0.005254669 & -0.058822445 & -0.041751032 \\
\hline 4.8 & 0 & 0.00517555 & -0.060296647 & -0.043215735 \\
\hline 4.9 & 0 & 0.005097275 & -0.061763328 & -0.044672916 \\
\hline 5 & 0 & 0.005019832 & -0.063222589 & -0.046122679 \\
\hline 5.1 & 0 & 0.004943211 & -0.06467453 & -0.047565122 \\
\hline 5.2 & 0 & 0.0048674 & -0.066119247 & -0.049000342 \\
\hline 5.3 & 0 & 0.004792388 & -0.067556833 & -0.050428432 \\
\hline 5.4 & 0 & 0.004718164 & -0.068987382 & -0.051849486 \\
\hline 5.5 & 0 & 0.00464472 & -0.070410983 & -0.053263591 \\
\hline 5.6 & 0 & 0.004572043 & -0.071827723 & -0.054670837 \\
\hline 5.7 & 0 & 0.004500125 & -0.073237688 & -0.056071308 \\
\hline 5. & 0 & 0.004428955 & -0.074640961 & -0.057465089 \\
\hline
\end{tabular}




\begin{tabular}{|c|c|c|c|c|}
\hline 5.9 & 0 & 0.004358525 & -0.076037625 & -0.058852261 \\
\hline 6 & 0 & 0.004288825 & -0.07742776 & -0.060232903 \\
\hline 6.1 & 0 & 0.004219846 & -0.078811442 & -0.061607095 \\
\hline 6.2 & 0 & 0.004151579 & -0.08018875 & -0.062974913 \\
\hline 6.3 & 0 & 0.004084015 & -0.081559757 & -0.064336431 \\
\hline 6.4 & 0 & 0.004017147 & -0.082924537 & -0.065691723 \\
\hline 6.5 & 0 & 0.003950965 & -0.084283162 & -0.06704086 \\
\hline 6.6 & 0 & 0.003885462 & -0.085635701 & -0.068383912 \\
\hline 6.7 & 0 & 0.003820629 & -0.086982224 & -0.069720949 \\
\hline 6.8 & 0 & 0.00375646 & -0.088322798 & -0.071052038 \\
\hline 6.9 & 0 & 0.003692946 & -0.089657488 & -0.072377243 \\
\hline 7 & 0 & 0.00363008 & -0.09098636 & -0.073696631 \\
\hline 7.1 & 0 & 0.003567853 & -0.092309476 & -0.075010265 \\
\hline 7.2 & 0 & 0.003506261 & -0.0936269 & -0.076318205 \\
\hline 7.3 & 0 & 0.003445295 & -0.09493869 & -0.077620515 \\
\hline 7.4 & 0 & 0.003384948 & -0.096244908 & -0.078917252 \\
\hline 7.5 & 0 & 0.003325213 & -0.097545613 & -0.080208477 \\
\hline 7.6 & 0 & 0.003266084 & -0.09884086 & -0.081494246 \\
\hline 7.7 & 0 & 0.003207555 & -0.100130708 & -0.082774615 \\
\hline 7.8 & 0 & 0.003149619 & -0.10141521 & -0.08404964 \\
\hline 7.9 & 0 & 0.003092269 & -0.102694423 & -0.085319376 \\
\hline 8 & 0 & 0.0030355 & -0.103968398 & -0.086583876 \\
\hline 8.1 & 0 & 0.002979306 & -0.105237189 & -0.087843192 \\
\hline 8.2 & 0 & 0.00292368 & -0.106500847 & -0.089097376 \\
\hline 8.3 & 0 & 0.002868616 & -0.107759422 & -0.090346477 \\
\hline 8.4 & 0 & 0.00281411 & -0.109012964 & -0.091590547 \\
\hline 8.5 & 0 & 0.002760155 & -0.110261522 & -0.092829634 \\
\hline 8.6 & 0 & 0.002706746 & -0.111505144 & -0.094063785 \\
\hline 8.7 & 0 & 0.002653877 & -0.112743877 & -0.095293049 \\
\hline 8.8 & 0 & 0.002601543 & -0.113977767 & -0.09651747 \\
\hline 8.9 & 0 & 0.002549739 & -0.11520686 & -0.097737096 \\
\hline 9 & 0 & 0.002498459 & -0.116431202 & -0.09895197 \\
\hline
\end{tabular}




\begin{tabular}{|c|c|c|c|c|}
\hline .1 & 0 & 0.002447699 & -0.117650835 & -0.100162137 \\
\hline 9.2 & 0 & 0.002397453 & -0.118865804 & -0.101367641 \\
\hline 9.3 & 0 & 0.002347717 & -0.12007615 & -0.102568523 \\
\hline 9.4 & & 0.002298485 & -0.121281916 & -0.103764826 \\
\hline 9.5 & 0 & 0.002249754 & -0.122483144 & -0.104956591 \\
\hline 9.6 & 0 & 0.002201518 & -0.123679874 & -0.106143859 \\
\hline 9.7 & 0 & 0.002153772 & -0.124872145 & -0.10732667 \\
\hline 9.8 & 0 & 0.002106513 & -0.126059998 & -0.108505063 \\
\hline 9.9 & 0 & 0.002059736 & -0.127243471 & -0.109679077 \\
\hline 10 & 0 & 0.002013436 & -0.128422602 & -0.11084875 \\
\hline 10.1 & 0 & 0.001967609 & -0.129597429 & -0.11201412 \\
\hline 10.2 & 0 & 0.00192225 & -0.130767989 & -0.113175224 \\
\hline 10.3 & 0 & 0.001877356 & -0.131934318 & -0.114332099 \\
\hline 10.4 & 0 & 0.001832923 & -0.133096453 & -0.115484779 \\
\hline 10.5 & 0 & 0.001788946 & -0.134254429 & -0.116633302 \\
\hline 10.6 & 0 & 0.001745422 & -0.13540828 & -0.117777701 \\
\hline 10.7 & 0 & 0.001702346 & -0.136558041 & -0.118918011 \\
\hline 10.8 & 0 & 0.001659715 & -0.137703747 & -0.120054266 \\
\hline 10.9 & 0 & 0.001617524 & -0.138845429 & -0.121186499 \\
\hline 11 & 0 & 0.001575771 & -0.139983122 & -0.122314744 \\
\hline 11.1 & 0 & 0.001534451 & -0.141116858 & -0.123439032 \\
\hline 11.2 & 0 & 0.00149356 & -0.142246668 & -0.124559396 \\
\hline 11.3 & 0 & 0.001453096 & -0.143372585 & -0.125675867 \\
\hline 11.4 & 0 & 0.001413054 & -0.144494638 & -0.126788477 \\
\hline 11.5 & 0 & 0.001373431 & -0.14561286 & -0.127897255 \\
\hline 11.6 & 0 & 0.001334224 & -0.146727281 & -0.129002233 \\
\hline 11.7 & 0 & 0.001295429 & -0.147837929 & -0.13010344 \\
\hline 11.8 & 0 & 0.001257042 & -0.148944836 & -0.131200906 \\
\hline 11.9 & 0 & 0.001219061 & -0.150048028 & -0.13229466 \\
\hline 12 & 0 & 0.001181483 & -0.151147537 & -0.13338473 \\
\hline 12.1 & 0 & 0.001144303 & -0.152243389 & -0.134471144 \\
\hline 12.2 & 0 & 0.001107519 & -0.153335612 & -0.135553931 \\
\hline
\end{tabular}




\begin{tabular}{|c|c|c|c|c|}
\hline 2.3 & 0 & 0.001071129 & -0.154424235 & -0.136633119 \\
\hline 12.4 & 0 & 0.001035127 & -0.155509284 & -0.137708734 \\
\hline 12.5 & 0 & 0.000999512 & -0.156590786 & -0.138780803 \\
\hline 12.6 & 0 & 0.000964281 & -0.157668768 & -0.139849352 \\
\hline 12.7 & 0 & 0.00092943 & -0.158743256 & -0.140914409 \\
\hline 12.8 & 0 & 0.000894958 & -0.159814275 & -0.141975998 \\
\hline 12.9 & 0 & 0.00086086 & -0.160881851 & -0.143034146 \\
\hline 13 & 0 & 0.000827133 & -0.16194601 & -0.144088877 \\
\hline 13.1 & 0 & 0.000793777 & -0.163006777 & -0.145140216 \\
\hline 13.2 & 0 & 0.000760786 & -0.164064175 & -0.146188189 \\
\hline 13.3 & 0 & 0.000728159 & -0.165118229 & -0.147232818 \\
\hline 13.4 & 0 & 0.000695893 & -0.166168963 & -0.148274129 \\
\hline 13.5 & 0 & 0.000663985 & -0.167216401 & -0.149312144 \\
\hline 13.6 & 0 & 0.000632432 & -0.168260566 & -0.150346887 \\
\hline 13.7 & 0 & 0.000601233 & -0.169301481 & -0.151378382 \\
\hline 13.8 & 0 & 0.000570384 & -0.170339169 & -0.152406651 \\
\hline 13.9 & 0 & 0.000539883 & -0.171373653 & -0.153431717 \\
\hline 14 & 0 & 0.000509728 & -0.172404955 & -0.154453602 \\
\hline 14.1 & 0 & 0.000479915 & -0.173433097 & -0.155472328 \\
\hline 14.2 & 0 & 0.000450442 & -0.174458101 & -0.156487916 \\
\hline 14.3 & 0 & 0.000421308 & -0.175479987 & -0.157500389 \\
\hline 14.4 & 0 & 0.000392509 & -0.176498779 & -0.158509768 \\
\hline 14.5 & 0 & 0.000364044 & -0.177514496 & -0.159516073 \\
\hline 14.6 & 0 & 0.000335909 & -0.178527159 & -0.160519326 \\
\hline 14.7 & 0 & 0.000308103 & -0.179536789 & -0.161519547 \\
\hline 14.8 & 0 & 0.000280623 & -0.180543407 & -0.162516756 \\
\hline 14.9 & 0 & 0.000253468 & -0.181547031 & -0.163510974 \\
\hline 15 & 0 & 0.000226634 & -0.182547684 & -0.16450222 \\
\hline 15.1 & 0 & 0.00020012 & -0.183545382 & -0.165490514 \\
\hline 15.2 & 0 & 0.000173924 & -0.184540148 & -0.166475876 \\
\hline 15.3 & 0 & 0.000148042 & -0.185531998 & -0.167458324 \\
\hline 15.4 & 0 & 0.000122474 & -0.186520954 & -0.168437878 \\
\hline
\end{tabular}




\begin{tabular}{|c|c|c|c|c|}
\hline 5.5 & 0 & 0.0000972174 & -0.187507032 & -0.169414556 \\
\hline 15.6 & 0 & 0.0000722695 & -0.188490253 & -0.170388377 \\
\hline 15.7 & 0 & 0.0000476285 & -0.189470633 & -0.17135936 \\
\hline 15.8 & 0 & 0.0000232924 & -0.190448193 & -0.172327523 \\
\hline 15.9 & 0 & -0.0000007406 & -0.191422949 & -0.173292883 \\
\hline 16 & 0 & -0.0000244727 & -0.192394919 & -0.174255459 \\
\hline 16.1 & 0 & -0.0000479059 & -0.193364121 & -0.175215268 \\
\hline 16.2 & 0 & -0.0000710421 & -0.194330573 & -0.176172327 \\
\hline 16.3 & 0 & -0.0000938831 & -0.195294291 & -0.177126655 \\
\hline 16.4 & 0 & -0.000116431 & -0.196255293 & -0.178078267 \\
\hline 16.5 & 0 & -0.000138687 & -0.197213596 & -0.179027181 \\
\hline 16.6 & 0 & -0.000160654 & -0.198169216 & -0.179973414 \\
\hline 16.7 & 0 & -0.000182334 & -0.19912217 & -0.180916982 \\
\hline 16.8 & 0 & -0.000203727 & -0.200072474 & -0.181857901 \\
\hline 16.9 & 0 & -0.000224837 & -0.201020145 & -0.182796188 \\
\hline 17 & 0 & -0.000245664 & -0.201965199 & -0.183731859 \\
\hline 17.1 & 0 & -0.000266211 & -0.202907651 & -0.18466493 \\
\hline 17.2 & 0 & -0.000286479 & -0.203847517 & -0.185595416 \\
\hline 17.3 & 0 & -0.00030647 & -0.204784813 & -0.186523333 \\
\hline 17.4 & 0 & -0.000326186 & -0.205719554 & -0.187448697 \\
\hline 17.5 & 0 & -0.000345628 & -0.206651756 & -0.188371522 \\
\hline 17.6 & 0 & -0.000364799 & -0.207581434 & -0.189291824 \\
\hline 17.7 & 0 & -0.000383699 & -0.208508602 & -0.190209618 \\
\hline 17.8 & 0 & -0.00040233 & -0.209433275 & -0.191124919 \\
\hline 17.9 & 0 & -0.000420695 & -0.210355469 & -0.192037741 \\
\hline 18 & 0 & -0.000438794 & -0.211275197 & -0.192948099 \\
\hline 18.1 & 0 & -0.000456629 & -0.212192475 & -0.193856007 \\
\hline 18.2 & 0 & -0.000474202 & -0.213107316 & -0.19476148 \\
\hline 18.3 & 0 & -0.000491514 & -0.214019734 & -0.195664531 \\
\hline 18.4 & 0 & -0.000508567 & -0.214929744 & -0.196565175 \\
\hline 18.5 & 0 & -0.000525363 & -0.215837359 & -0.197463426 \\
\hline 18.6 & 0 & -0.000541902 & -0.216742593 & -0.198359297 \\
\hline
\end{tabular}




$\begin{array}{lllll}18.7 & 0 & -0.000558187 & -0.217645459 & -0.199252802 \\ 18.8 & 0 & -0.000574219 & -0.218545972 & -0.200143955 \\ 18.9 & 0 & -0.000589999 & -0.219444144 & -0.201032768 \\ 19 & 0 & -0.000605529 & -0.220339989 & -0.201919255 \\ 19.1 & 0 & -0.00062081 & -0.22123352 & -0.202803429 \\ 19.2 & 0 & -0.000635843 & -0.22212475 & -0.203685303 \\ 19.3 & 0 & -0.000650631 & -0.223013691 & -0.20456489 \\ 19.4 & 0 & -0.000665174 & -0.223900357 & -0.205442203 \\ 19.5 & 0 & -0.000679475 & -0.22478476 & -0.206317255 \\ 19.6 & 0 & -0.000693533 & -0.225666912 & -0.207190057 \\ 19.7 & 0 & -0.000707351 & -0.226546827 & -0.208060622 \\ 19.8 & 0 & -0.00072093 & -0.227424515 & -0.208928963 \\ 19.9 & 0 & -0.000734271 & -0.22829999 & -0.209795091 \\ 20 & 0 & -0.000747376 & -0.229173264 & -0.21065902\end{array}$

The Gibbs free energy difference of the four polytypes of boron nitride ( $r-\mathrm{BN}, \mathrm{h}-\mathrm{BN}, \mathrm{c}-\mathrm{BN}$, and w-BN) as a function of pressure ranging from 0 to $20 \mathrm{GPa}$, calculated with respect to $\mathrm{r}-\mathrm{BN}$ as a reference at a given temperature of $\mathbf{7 0 0}$ Kelvin. The unit of the Gibbs free energy difference for the four polytypes of boron nitride is given in $\mathrm{eV} / \mathrm{atom}$.

$\begin{array}{lllll}\mathbf{P}(\mathbf{G P a}) \mathbf{r}-\mathbf{B N} & \mathbf{h}-\mathbf{B N} & \mathbf{c}-\mathbf{B N} & \mathbf{w}-\mathbf{B N} \\ 0 & 0 & 0.011353212 & 0.026677401 & 0.04317082 \\ 0.1 & 0 & 0.011198914 & 0.024628819 & 0.041131781 \\ 0.2 & 0 & 0.011046571 & 0.022600385 & 0.03911289 \\ 0.3 & 0 & 0.010896156 & 0.020591409 & 0.037113458 \\ 0.4 & 0 & 0.010747644 & 0.018601253 & 0.035132844 \\ 0.5 & 0 & 0.010601004 & 0.016629316 & 0.03317045 \\ 0.6 & 0 & 0.010456207 & 0.01467504 & 0.031225716 \\ 0.7 & 0 & 0.010313222 & 0.012737898 & 0.029298116 \\ 0.8 & 0 & 0.010172019 & 0.010817397 & 0.027387156 \\ 0.9 & 0 & 0.010032565 & 0.008913068 & 0.025492369 \\ 1 & 0 & 0.00989483 & 0.007024473 & 0.023613315 \\ 1.1 & 0 & 0.009758783 & 0.005151196 & 0.021749577\end{array}$




\begin{tabular}{|c|c|c|c|c|}
\hline 1.2 & 0 & 0.009624395 & 0.00329284 & 0.019900762 \\
\hline 1.3 & 0 & 0.009491635 & 0.001449033 & 0.018066494 \\
\hline 1.4 & 0 & 0.009360474 & -0.000380584 & 0.016246417 \\
\hline 1.5 & 0 & 0.009230884 & -0.002196347 & 0.014440192 \\
\hline 1.6 & 0 & 0.009102836 & -0.003998581 & 0.012647496 \\
\hline 1.7 & 0 & 0.008976303 & -0.005787594 & 0.010868021 \\
\hline 1.8 & 0 & 0.008851258 & -0.007563681 & 0.009101472 \\
\hline 1.9 & 0 & 0.008727675 & -0.009327123 & 0.007347567 \\
\hline 2 & 0 & 0.008605529 & -0.01107819 & 0.005606036 \\
\hline 2.1 & 0 & 0.008484795 & -0.012817141 & 0.003876621 \\
\hline 2.2 & 0 & 0.008365448 & -0.014544225 & 0.002159074 \\
\hline 2.3 & 0 & 0.008247465 & -0.016259679 & 0.000453154 \\
\hline 2.4 & 0 & 0.008130824 & -0.017963733 & -0.001241365 \\
\hline 2.5 & 0 & 0.008015501 & -0.019656608 & -0.002924705 \\
\hline 2.6 & 0 & 0.007901476 & -0.021338515 & -0.004597079 \\
\hline 2.7 & 0 & 0.007788726 & -0.02300966 & -0.00625869 \\
\hline 2.8 & 0 & 0.007677231 & -0.024670239 & -0.007909736 \\
\hline 2.9 & 0 & 0.007566971 & -0.026320442 & -0.009550407 \\
\hline 3 & 0 & 0.007457927 & -0.027960454 & -0.011180887 \\
\hline 3.1 & 0 & 0.007350079 & -0.029590453 & -0.012801354 \\
\hline 3.2 & 0 & 0.007243409 & -0.031210609 & -0.01441198 \\
\hline 3.3 & 0 & 0.007137899 & -0.032821089 & -0.01601293 \\
\hline 3.4 & 0 & 0.00703353 & -0.034422053 & -0.017604365 \\
\hline 3.5 & 0 & 0.006930286 & -0.036013659 & -0.019186441 \\
\hline 3.6 & 0 & 0.006828151 & -0.037596055 & -0.020759309 \\
\hline 3.7 & 0 & 0.006727107 & -0.039169389 & -0.022323115 \\
\hline 3.8 & 0 & 0.006627138 & -0.040733803 & -0.023878001 \\
\hline 3.9 & 0 & 0.00652823 & -0.042289435 & -0.025424106 \\
\hline 4 & 0 & 0.006430367 & -0.043836417 & -0.026961562 \\
\hline 4.1 & 0 & 0.006333534 & -0.045374881 & -0.0284905 \\
\hline 4.2 & 0 & 0.006237716 & -0.046904952 & -0.030011046 \\
\hline 4.3 & 0 & 0.0061429 & -0.048426753 & -0.031523323 \\
\hline
\end{tabular}




\begin{tabular}{|c|c|c|c|c|}
\hline & 0 & 0.006049072 & -0.049940404 & -0.03302745 \\
\hline 4.5 & 0 & 0.005956218 & -0.051446021 & -0.034523544 \\
\hline 4.6 & 0 & 0.005864325 & -0.052943717 & -0.036011717 \\
\hline 47 & & 0.005773381 & -0.054433602 & -0.037492081 \\
\hline 4.8 & 0 & 0.005683372 & -0.055915784 & -0.038964742 \\
\hline 4.9 & 0 & 0.005594287 & -0.057390367 & -0.040429804 \\
\hline 5 & 0 & 0.005506113 & -0.058857453 & -0.04188737 \\
\hline 5.1 & 0 & 0.005418839 & -0.060317142 & -0.04333754 \\
\hline 5.2 & 0 & 0.005332453 & -0.061769531 & -0.04478041 \\
\hline 5.3 & 0 & 0.005246945 & -0.063214714 & -0.046216075 \\
\hline 5.4 & 0 & 0.005162302 & -0.064652784 & -0.047644628 \\
\hline 5.5 & 0 & 0.005078515 & -0.066083831 & -0.049066158 \\
\hline 5.6 & 0 & 0.004995572 & -0.067507943 & -0.050480754 \\
\hline 5.7 & 0 & 0.004913464 & -0.068925206 & -0.051888502 \\
\hline 5.8 & 0 & 0.004832181 & -0.070335705 & -0.053289486 \\
\hline 5.9 & 0 & 0.004751712 & -0.071739521 & -0.054683789 \\
\hline 6 & 0 & 0.004672047 & -0.073136736 & -0.056071491 \\
\hline 6.1 & 0 & 0.004593179 & -0.074527427 & -0.05745267 \\
\hline 6.2 & 0 & 0.004515096 & -0.075911673 & -0.058827404 \\
\hline 6.3 & 0 & 0.004437791 & -0.077289547 & -0.060195768 \\
\hline 6.4 & 0 & 0.004361253 & -0.078661125 & -0.061557835 \\
\hline 6.5 & 0 & 0.004285475 & -0.080026477 & -0.062913678 \\
\hline 6.6 & 0 & 0.004210448 & -0.081385675 & -0.064263368 \\
\hline 6.7 & 0 & 0.004136163 & -0.082738788 & -0.065606973 \\
\hline 6.8 & 0 & 0.004062613 & -0.084085884 & -0.066944562 \\
\hline 6.9 & 0 & 0.003989788 & -0.085427029 & -0.0682762 \\
\hline 7 & 0 & 0.003917682 & -0.086762289 & -0.069601955 \\
\hline 7.1 & 0 & 0.003846286 & -0.088091726 & -0.070921888 \\
\hline 7.2 & 0 & 0.003775593 & -0.089415405 & -0.072236062 \\
\hline 7.3 & 0 & 0.003705595 & -0.090733386 & -0.07354454 \\
\hline 7.4 & 0 & 0.003636285 & -0.092045729 & -0.074847381 \\
\hline 7.5 & 0 & 0.003567656 & -0.093352493 & -0.076144644 \\
\hline
\end{tabular}




\begin{tabular}{|c|c|c|c|c|}
\hline 7.6 & 0 & 0.003499701 & -0.094653738 & -0.077436388 \\
\hline 7.7 & 0 & 0.003432412 & -0.095949518 & -0.078722669 \\
\hline 7.8 & 0 & 0.003365782 & -0.097239891 & -0.080003543 \\
\hline 7.9 & 0 & 0.003299806 & -0.098524911 & -0.081279065 \\
\hline 8 & 0 & 0.003234477 & -0.099804632 & -0.082549288 \\
\hline 8.1 & 0 & 0.003169787 & -0.101079107 & -0.083814266 \\
\hline 8.2 & 0 & 0.003105731 & -0.102348387 & -0.085074051 \\
\hline 8.3 & 0 & 0.003042302 & -0.103612524 & -0.086328694 \\
\hline 8.4 & 0 & 0.002979494 & -0.104871568 & -0.087578244 \\
\hline 8.5 & 0 & 0.002917302 & -0.106125569 & -0.088822751 \\
\hline 8.6 & 0 & 0.002855719 & -0.107374574 & -0.090062264 \\
\hline 8.7 & 0 & 0.002794739 & -0.108618631 & -0.09129683 \\
\hline 8.8 & 0 & 0.002734356 & -0.109857787 & -0.092526496 \\
\hline 8.9 & 0 & 0.002674565 & -0.111092088 & -0.093751308 \\
\hline 9 & 0 & 0.002615361 & -0.11232158 & -0.094971311 \\
\hline 9.1 & 0 & 0.002556737 & -0.113546306 & -0.09618655 \\
\hline 9.2 & 0 & 0.002498689 & -0.114766311 & -0.097397068 \\
\hline 9.3 & 0 & 0.002441211 & -0.115981637 & -0.098602909 \\
\hline 9.4 & 0 & 0.002384297 & -0.117192328 & -0.099804114 \\
\hline 9.5 & 0 & 0.002327944 & -0.118398424 & -0.101000727 \\
\hline 9.6 & 0 & 0.002272145 & -0.119599968 & -0.102192787 \\
\hline 9.7 & 0 & 0.002216896 & -0.120796998 & -0.103380336 \\
\hline 9.8 & 0 & 0.002162191 & -0.121989556 & -0.104563413 \\
\hline 9.9 & 0 & 0.002108027 & -0.12317768 & -0.105742056 \\
\hline 10 & 0 & 0.002054397 & -0.124361409 & -0.106916306 \\
\hline 10.1 & 0 & 0.002001298 & -0.125540781 & -0.1080862 \\
\hline 10.2 & 0 & 0.001948725 & -0.126715833 & -0.109251774 \\
\hline 10.3 & 0 & 0.001896673 & -0.127886602 & -0.110413067 \\
\hline 10.4 & 0 & 0.001845138 & -0.129053125 & -0.111570115 \\
\hline 10.5 & 0 & 0.001794116 & -0.130215437 & -0.112722952 \\
\hline 10.6 & 0 & 0.001743601 & -0.131373573 & -0.113871615 \\
\hline 10.7 & 0 & 0.00169359 & -0.132527569 & -0.115016138 \\
\hline
\end{tabular}




\begin{tabular}{|c|c|c|c|c|}
\hline 10.8 & 0 & 0.001644079 & -0.133677458 & -0.116156556 \\
\hline 10.9 & 0 & 0.001595063 & -0.134823274 & -0.117292902 \\
\hline 11 & 0 & 0.001546538 & -0.135965051 & -0.118425209 \\
\hline 11.1 & 0 & 0.001498501 & -0.137102821 & -0.119553511 \\
\hline 11.2 & 0 & 0.001450946 & -0.138236617 & -0.12067784 \\
\hline 11.3 & 0 & 0.001403871 & -0.139366471 & -0.121798227 \\
\hline 11.4 & 0 & 0.001357271 & -0.140492413 & -0.122914704 \\
\hline 11.5 & 0 & 0.001311143 & -0.141614476 & -0.124027303 \\
\hline 11.6 & 0 & 0.001265483 & -0.142732689 & -0.125136053 \\
\hline 11.7 & 0 & 0.001220286 & -0.143847083 & -0.126240984 \\
\hline 11.8 & 0 & 0.00117555 & -0.144957688 & -0.127342128 \\
\hline 11.9 & 0 & 0.001131271 & -0.146064533 & -0.128439513 \\
\hline 12 & 0 & 0.001087445 & -0.147167647 & -0.129533167 \\
\hline 12.1 & 0 & 0.001044069 & -0.148267058 & -0.130623121 \\
\hline 12.2 & 0 & 0.001001138 & -0.149362795 & -0.131709401 \\
\hline 12.3 & 0 & 0.000958651 & -0.150454886 & -0.132792036 \\
\hline 12.4 & 0 & 0.000916602 & -0.151543358 & -0.133871053 \\
\hline 12.5 & 0 & 0.00087499 & -0.152628239 & -0.13494648 \\
\hline 12.6 & 0 & 0.00083381 & -0.153709554 & -0.136018343 \\
\hline 12.7 & 0 & 0.00079306 & -0.154787332 & -0.137086669 \\
\hline 12.8 & 0 & 0.000752736 & -0.155861597 & -0.138151483 \\
\hline 12.9 & 0 & 0.000712835 & -0.156932375 & -0.139212812 \\
\hline 13 & 0 & 0.000673354 & -0.157999693 & -0.140270682 \\
\hline 13.1 & 0 & 0.00063429 & -0.159063575 & -0.141325116 \\
\hline 13.2 & 0 & 0.000595639 & -0.160124046 & -0.142376141 \\
\hline 13.3 & 0 & 0.000557399 & -0.16118113 & -0.14342378 \\
\hline 13.4 & 0 & 0.000519567 & -0.162234852 & -0.144468059 \\
\hline 13.5 & 0 & 0.000482139 & -0.163285236 & -0.145509 \\
\hline 13.6 & 0 & 0.000445114 & -0.164332306 & -0.146546628 \\
\hline 13.7 & 0 & 0.000408487 & -0.165376084 & -0.147580966 \\
\hline 13.8 & 0 & 0.000372256 & -0.166416594 & -0.148612037 \\
\hline 13.9 & 0 & 0.000336419 & -0.167453859 & -0.149639863 \\
\hline
\end{tabular}




\begin{tabular}{|c|c|c|c|c|}
\hline 14 & 0 & 0.000300972 & -0.168487902 & -0.150664469 \\
\hline 14.1 & 0 & 0.000265913 & -0.169518743 & -0.151685875 \\
\hline 14.2 & 0 & 0.00023124 & -0.170546407 & -0.152704103 \\
\hline 14.3 & 0 & 0.000196948 & -0.171570914 & -0.153719177 \\
\hline 14.4 & 0 & 0.000163037 & -0.172592286 & -0.154731116 \\
\hline 14.5 & 0 & 0.000129502 & -0.173610544 & -0.155739943 \\
\hline 14.6 & 0 & 0.0000963422 & -0.17462571 & -0.156745678 \\
\hline 14.7 & 0 & 0.0000635543 & -0.175637803 & -0.157748343 \\
\hline 14.8 & 0 & 0.000031136 & -0.176646845 & -0.158747957 \\
\hline 14.9 & 0 & -0.0000009155 & -0.177652856 & -0.159744542 \\
\hline 15 & 0 & -0.0000326023 & -0.178655857 & -0.160738116 \\
\hline 15.1 & 0 & -0.0000639271 & -0.179655866 & -0.161728701 \\
\hline 5.2 & 0 & -0.0000948921 & -0.180652904 & -0.162716316 \\
\hline 15.3 & 0 & -0.0001255 & -0.181646989 & -0.16370098 \\
\hline 15.4 & 0 & -0.000155753 & -0.182638143 & -0.164682713 \\
\hline 15.5 & 0 & -0.000185653 & -0.183626382 & -0.165661533 \\
\hline 15.6 & 0 & -0.000215203 & -0.184611727 & -0.166637459 \\
\hline 15.7 & 0 & -0.000244405 & -0.185594196 & -0.167610511 \\
\hline 15.8 & 0 & -0.000273261 & -0.186573806 & -0.168580706 \\
\hline 15.9 & 0 & -0.000301774 & -0.187550578 & -0.169548062 \\
\hline 16 & 0 & -0.000329945 & -0.188524527 & -0.170512598 \\
\hline 16.1 & 0 & -0.000357777 & -0.189495673 & -0.171474332 \\
\hline 16.2 & 0 & -0.000385272 & -0.190464033 & -0.172433281 \\
\hline 16.3 & 0 & -0.000412432 & -0.191429624 & -0.173389463 \\
\hline 16.4 & 0 & -0.00043926 & -0.192392465 & -0.174342895 \\
\hline 16.5 & 0 & -0.000465757 & -0.193352571 & -0.175293593 \\
\hline 16.6 & 0 & -0.000491925 & -0.194309959 & -0.176241576 \\
\hline 16.7 & 0 & -0.000517767 & -0.195264647 & -0.17718686 \\
\hline 16.8 & 0 & -0.000543284 & -0.196216652 & -0.17812946 \\
\hline 16.9 & 0 & -0.000568479 & -0.197165989 & -0.179069395 \\
\hline 17 & 0 & -0.000593353 & -0.198112674 & -0.18000668 \\
\hline 17.1 & 0 & -0.000617909 & -0.199056725 & -0.180941331 \\
\hline
\end{tabular}




\begin{tabular}{|c|c|c|c|c|}
\hline 17.2 & 0 & -0.000642148 & -0.199998156 & -0.181873363 \\
\hline 17.3 & 0 & -0.000666072 & -0.200936984 & -0.182802794 \\
\hline 17.4 & 0 & -0.000689684 & -0.201873224 & -0.183729639 \\
\hline 17.5 & 0 & -0.000712984 & -0.202806892 & -0.184653912 \\
\hline 17.6 & 0 & -0.000735976 & -0.203738003 & -0.185575629 \\
\hline 17.7 & 0 & -0.00075866 & -0.204666571 & -0.186494806 \\
\hline 17.8 & 0 & -0.00078104 & -0.205592613 & -0.187411457 \\
\hline 17.9 & 0 & -0.000803115 & -0.206516143 & -0.188325598 \\
\hline 18 & 0 & -0.000824889 & -0.207437176 & -0.189237242 \\
\hline 18.1 & 0 & -0.000846363 & -0.208355726 & -0.190146406 \\
\hline 18.2 & 0 & -0.000867538 & -0.209271808 & -0.191053102 \\
\hline 18.3 & 0 & -0.000888417 & -0.210185436 & -0.191957345 \\
\hline 18.4 & 0 & -0.000909002 & -0.211096624 & -0.19285915 \\
\hline 18.5 & 0 & -0.000929293 & -0.212005386 & -0.193758531 \\
\hline 18.6 & 0 & -0.000949293 & -0.212911736 & -0.194655501 \\
\hline 18.7 & 0 & -0.000969003 & -0.213815689 & -0.195550075 \\
\hline 18.8 & 0 & -0.000988425 & -0.214717256 & -0.196442265 \\
\hline 18.9 & 0 & -0.001007561 & -0.215616453 & -0.197332085 \\
\hline 19 & 0 & -0.001026412 & -0.216513293 & -0.19821955 \\
\hline 19.1 & 0 & -0.00104498 & -0.217407788 & -0.199104672 \\
\hline 19.2 & 0 & -0.001063266 & -0.218299952 & -0.199987463 \\
\hline 19.3 & 0 & -0.001081272 & -0.219189798 & -0.200867938 \\
\hline 19.4 & 0 & -0.001099 & -0.220077339 & -0.20174611 \\
\hline 19.5 & 0 & -0.001116451 & -0.220962588 & -0.20262199 \\
\hline 19.6 & 0 & -0.001133627 & -0.221845557 & -0.203495592 \\
\hline 19.7 & 0 & -0.001150529 & -0.222726258 & -0.204366928 \\
\hline 19.8 & 0 & -0.001167158 & -0.223604705 & -0.205236011 \\
\hline 19.9 & 0 & -0.001183517 & -0.22448091 & -0.206102852 \\
\hline & 0 & -0.001199606 & -0.225354884 & -0.206967465 \\
\hline
\end{tabular}


The Gibbs free energy difference of the four polytypes of boron nitride ( $\mathrm{r}-\mathrm{BN}, \mathrm{h}-\mathrm{BN}, \mathrm{c}-\mathrm{BN}$, and w-BN) as a function of pressure ranging from 0 to $20 \mathrm{GPa}$, calculated with respect to $\mathrm{r}-\mathrm{BN}$ as a reference at a given temperature of 800 Kelvin. The unit of the Gibbs free energy difference for the four polytypes of boron nitride is given in $\mathrm{eV} / \mathrm{atom}$.

\begin{tabular}{lllll}
$\mathbf{P}(\mathbf{G P a}) \mathbf{r}-\mathbf{B N}$ & $\mathbf{h}-\mathbf{B N}$ & $\mathbf{c}-\mathbf{B N}$ & $\mathbf{w}-\mathbf{B N}$ \\
0 & 0 & 0.012519656 & 0.031471049 & 0.047831823 \\
0.1 & 0 & 0.01235208 & 0.029412615 & 0.045782863 \\
0.2 & 0 & 0.012186312 & 0.027374209 & 0.04375393 \\
0.3 & 0 & 0.012022352 & 0.025355167 & 0.041744361 \\
0.4 & 0 & 0.011860197 & 0.023354869 & 0.039753536 \\
0.5 & 0 & 0.01169984 & 0.021372735 & 0.037780875 \\
0.6 & 0 & 0.011541268 & 0.019408221 & 0.035825833 \\
0.7 & 0 & 0.011384467 & 0.017460814 & 0.033887898 \\
0.8 & 0 & 0.011229419 & 0.015530033 & 0.031966589 \\
0.9 & 0 & 0.011076105 & 0.013615421 & 0.030061449 \\
1 & 0 & 0.010924507 & 0.011716549 & 0.028172049 \\
1.1 & 0 & 0.010774602 & 0.009833008 & 0.02629798 \\
1.2 & 0 & 0.010626369 & 0.007964411 & 0.024438854 \\
1.3 & 0 & 0.010479787 & 0.006110391 & 0.022594304 \\
1.4 & 0 & 0.010334831 & 0.004270595 & 0.020763979 \\
1.5 & 0 & 0.010191481 & 0.002444692 & 0.018947546 \\
1.6 & 0 & 0.010049713 & 0.000632362 & 0.017144686 \\
1.7 & 0 & 0.009909505 & -0.001166699 & 0.015355095 \\
1.8 & 0 & 0.009770834 & -0.002952783 & 0.013578481 \\
1.9 & 0 & 0.009633678 & -0.004726166 & 0.011814567 \\
2 & 0 & 0.009498015 & -0.006487118 & 0.010063085 \\
2.1 & 0 & 0.009363823 & -0.008235893 & 0.008323778 \\
2.2 & 0 & 0.009231081 & -0.009972738 & 0.006596402 \\
2.3 & 0 & 0.009099768 & -0.011697889 & 0.004880719 \\
2.4 & 0 & 0.008969863 & -0.013411574 & 0.003176502 \\
\hline .5 & 0 & 0.008841344 & -0.015114011 & 0.001483532 \\
\hline & 0 & 0.008714194 & -0.016805412 & -0.000198402
\end{tabular}




\begin{tabular}{|c|c|c|c|c|}
\hline 2.7 & 0 & 0.008588391 & -0.01848598 & -0.001869503 \\
\hline 2.8 & 0 & 0.008463916 & -0.020155911 & -0.003529968 \\
\hline 2.9 & 0 & 0.008340751 & -0.021815395 & -0.005179985 \\
\hline 3 & 0 & 0.008218876 & -0.023464614 & -0.006819739 \\
\hline 3.1 & 0 & 0.008098275 & -0.025103746 & -0.008449405 \\
\hline 3.2 & 0 & 0.007978928 & -0.026732961 & -0.010069155 \\
\hline 3.3 & 0 & 0.007860819 & -0.028352425 & -0.011679155 \\
\hline 3.4 & 0 & 0.00774393 & -0.0299623 & -0.013279565 \\
\hline 3.5 & 0 & 0.007628245 & -0.031562739 & -0.014870541 \\
\hline 3.6 & 0 & 0.007513746 & -0.033153894 & -0.016452233 \\
\hline 3.7 & 0 & 0.00740042 & -0.034735912 & -0.018024787 \\
\hline 3.8 & 0 & 0.007288248 & -0.036308933 & -0.019588346 \\
\hline 3.9 & 0 & 0.007177217 & -0.037873096 & -0.021143047 \\
\hline 4 & 0 & 0.007067311 & -0.039428534 & -0.022689023 \\
\hline 4.1 & 0 & 0.006958515 & -0.040975378 & -0.024226405 \\
\hline 4.2 & 0 & 0.006850816 & -0.042513753 & -0.02575532 \\
\hline 4.3 & 0 & 0.006744197 & -0.044043783 & -0.02727589 \\
\hline 4.4 & 0 & 0.006638647 & -0.045565588 & -0.028788234 \\
\hline 4.5 & 0 & 0.006534152 & -0.047079283 & -0.03029247 \\
\hline 4.6 & 0 & 0.006430697 & -0.048584982 & -0.03178871 \\
\hline 4.7 & 0 & 0.006328271 & -0.050082796 & -0.033277065 \\
\hline 4.8 & 0 & 0.00622686 & -0.051572832 & -0.034757643 \\
\hline 4.9 & 0 & 0.006126452 & -0.053055195 & -0.036230549 \\
\hline F & 0 & 0.006027035 & -0.054529987 & -0.037695884 \\
\hline 5.1 & 0 & 0.005928597 & -0.055997309 & -0.039153748 \\
\hline 5.2 & 0 & 0.005831125 & -0.057457256 & -0.04060424 \\
\hline 5.3 & 0 & 0.005734609 & -0.058909926 & -0.042047454 \\
\hline 5.4 & 0 & 0.005639038 & -0.060355409 & -0.043483482 \\
\hline 5.5 & 0 & 0.005544399 & -0.061793798 & -0.044912416 \\
\hline 5.6 & 0 & 0.005450683 & -0.063225179 & -0.046334344 \\
\hline 5.7 & 0 & 0.005357879 & -0.064649641 & -0.047749352 \\
\hline 5.8 & 0 & 0.005265976 & -0.066067267 & -0.049157525 \\
\hline
\end{tabular}




\begin{tabular}{|c|c|c|c|c|}
\hline 5.9 & 0 & 0.005174964 & -0.067478141 & -0.050558946 \\
\hline 6 & 0 & 0.005084832 & -0.068882342 & -0.051953695 \\
\hline 6.1 & 0 & 0.004995573 & -0.07027995 & -0.053341852 \\
\hline 6.2 & 0 & 0.004907174 & -0.071671043 & -0.054723494 \\
\hline 6.3 & 0 & 0.004819628 & -0.073055696 & -0.056098697 \\
\hline 6.4 & 0 & 0.004732924 & -0.074433983 & -0.057467534 \\
\hline 6.5 & 0 & 0.004647054 & -0.075805977 & -0.058830079 \\
\hline 6.6 & 0 & 0.004562009 & -0.077171749 & -0.060186403 \\
\hline 6.7 & 0 & 0.00447778 & -0.078531369 & -0.061536575 \\
\hline 6.8 & 0 & 0.004394357 & -0.079884905 & -0.062880664 \\
\hline 6.9 & 0 & 0.004311734 & -0.081232424 & -0.064218736 \\
\hline 7 & 0 & 0.004229901 & -0.082573991 & -0.065550857 \\
\hline 7.1 & 0 & 0.00414885 & -0.083909671 & -0.066877091 \\
\hline 7.2 & 0 & 0.004068573 & -0.085239526 & -0.068197502 \\
\hline 7.3 & 0 & 0.003989062 & -0.08656362 & -0.069512151 \\
\hline 7.4 & 0 & 0.00391031 & -0.087882011 & -0.070821098 \\
\hline 7.5 & 0 & 0.003832308 & -0.08919476 & -0.072124405 \\
\hline 7.6 & 0 & 0.00375505 & -0.090501925 & -0.073422127 \\
\hline 7.7 & 0 & 0.003678528 & -0.091803564 & -0.074714324 \\
\hline 7.8 & 0 & 0.003602735 & -0.093099732 & -0.076001052 \\
\hline 7.9 & 0 & 0.003527663 & -0.094390485 & -0.077282364 \\
\hline 8 & 0 & 0.003453305 & -0.095675878 & -0.078558317 \\
\hline 8.1 & 0 & 0.003379655 & -0.096955963 & -0.079828963 \\
\hline 8.2 & 0 & 0.003306706 & -0.098230793 & -0.081094355 \\
\hline 8.3 & 0 & 0.003234451 & -0.099500419 & -0.082354543 \\
\hline 8.4 & 0 & 0.003162884 & -0.100764893 & -0.08360958 \\
\hline 8.5 & 0 & 0.003091998 & -0.102024262 & -0.084859513 \\
\hline 8.6 & 0 & 0.003021786 & -0.103278578 & -0.086104393 \\
\hline 8.7 & 0 & 0.002952243 & -0.104527886 & -0.087344266 \\
\hline 8.8 & 0 & 0.002883363 & -0.105772236 & -0.088579181 \\
\hline 8.9 & 0 & 0.002815138 & -0.107011672 & -0.089809184 \\
\hline 9 & 0 & 0.002747564 & -0.108246241 & -0.09103432 \\
\hline
\end{tabular}




\begin{tabular}{|c|c|c|c|c|}
\hline .1 & 0 & 0.002680635 & -0.109475988 & -0.092254634 \\
\hline 9.2 & 0 & 0.002614343 & -0.110700956 & -0.093470172 \\
\hline 9.3 & 0 & 0.002548685 & -0.11192119 & -0.094680975 \\
\hline 9.4 & & 0.002483654 & -0.113136732 & -0.095887086 \\
\hline 9.5 & 0 & 0.002419245 & -0.114347624 & -0.097088549 \\
\hline 9.6 & 0 & 0.002355452 & -0.115553907 & -0.098285404 \\
\hline 9.7 & 0 & 0.002292271 & -0.116755623 & -0.099477693 \\
\hline 9.8 & 0 & 0.002229695 & -0.117952812 & -0.100665454 \\
\hline 9.9 & 0 & 0.002167719 & -0.119145513 & -0.101848729 \\
\hline 10 & 0 & 0.002106339 & -0.120333765 & -0.103027555 \\
\hline 10.1 & 0 & 0.002045548 & -0.121517606 & -0.104201971 \\
\hline 10.2 & 0 & 0.001985344 & -0.122697074 & -0.105372016 \\
\hline 10.3 & 0 & 0.001925719 & -0.123872207 & -0.106537726 \\
\hline 10.4 & 0 & 0.001866671 & -0.125043042 & -0.107699137 \\
\hline 10.5 & 0 & 0.001808192 & -0.126209613 & -0.108856287 \\
\hline 10.6 & 0 & 0.00175028 & -0.127371957 & -0.11000921 \\
\hline 10.7 & 0 & 0.00169293 & -0.128530109 & -0.111157942 \\
\hline 10.8 & 0 & 0.001636136 & -0.129684104 & -0.112302518 \\
\hline 10.9 & 0 & 0.001579894 & -0.130833976 & -0.113442971 \\
\hline 11 & 0 & 0.0015242 & -0.131979757 & -0.114579335 \\
\hline 11.1 & 0 & 0.00146905 & -0.133121482 & -0.115711643 \\
\hline 11.2 & 0 & 0.001414439 & -0.134259184 & -0.116839928 \\
\hline 11.3 & 0 & 0.001360363 & -0.135392893 & -0.117964222 \\
\hline 11.4 & 0 & 0.001306817 & -0.136522643 & -0.119084558 \\
\hline 11.5 & 0 & 0.001253798 & -0.137648464 & -0.120200965 \\
\hline 11.6 & 0 & 0.001201301 & -0.138770388 & -0.121313476 \\
\hline 11.7 & 0 & 0.001149323 & -0.139888444 & -0.12242212 \\
\hline 11.8 & 0 & 0.001097859 & -0.141002663 & -0.123526929 \\
\hline 11.9 & 0 & 0.001046905 & -0.142113075 & -0.12462793 \\
\hline 12 & 0 & 0.000996458 & -0.143219709 & -0.125725155 \\
\hline 12.1 & 0 & 0.000946513 & -0.144322594 & -0.126818631 \\
\hline 12.2 & 0 & 0.000897068 & -0.145421759 & -0.127908388 \\
\hline
\end{tabular}




\begin{tabular}{|c|c|c|c|c|}
\hline 2.3 & 0 & 0.000848117 & -0.14651723 & -0.128994453 \\
\hline 12.4 & 0 & 0.000799658 & -0.147609038 & -0.130076854 \\
\hline 12.5 & 0 & 0.000751686 & -0.148697208 & -0.131155619 \\
\hline 12.6 & 0 & 0.000704199 & -0.149781768 & -0.132230774 \\
\hline 12.7 & 0 & 0.000657192 & -0.150862744 & -0.133302348 \\
\hline 2.8 & 0 & 0.000610662 & -0.151940164 & -0.134370365 \\
\hline 12.9 & 0 & 0.000564606 & -0.153014053 & -0.135434852 \\
\hline 13 & 0 & 0.00051902 & -0.154084437 & -0.136495835 \\
\hline 13.1 & 0 & 0.000473901 & -0.155151341 & -0.13755334 \\
\hline 13.2 & 0 & 0.000429245 & -0.156214791 & -0.13860739 \\
\hline 13.3 & 0 & 0.00038505 & -0.157274811 & -0.139658012 \\
\hline 13.4 & 0 & 0.000341311 & -0.158331426 & -0.14070523 \\
\hline 13.5 & 0 & 0.000298026 & -0.15938466 & -0.141749068 \\
\hline 13.6 & 0 & 0.000255191 & -0.160434536 & -0.142789549 \\
\hline 13.7 & 0 & 0.000212803 & -0.16148108 & -0.143826698 \\
\hline 13.8 & 0 & 0.00017086 & -0.162524313 & -0.144860538 \\
\hline 13.9 & 0 & 0.000129358 & -0.16356426 & -0.145891092 \\
\hline 14 & 0 & 0.0000882938 & -0.164600942 & -0.146918383 \\
\hline 14.1 & 0 & 0.0000476647 & -0.165634383 & -0.147942433 \\
\hline 14.2 & 0 & 0.0000074677 & -0.166664605 & -0.148963265 \\
\hline 14.3 & 0 & -0.0000323002 & -0.167691629 & -0.149980901 \\
\hline 14.4 & 0 & -0.0000716419 & -0.168715478 & -0.150995362 \\
\hline 14.5 & 0 & -0.00011056 & -0.169736173 & -0.15200667 \\
\hline 14.6 & 0 & -0.000149058 & -0.170753736 & -0.153014846 \\
\hline 14.7 & 0 & -0.000187138 & -0.171768187 & -0.154019912 \\
\hline 14.8 & 0 & -0.000224803 & -0.172779547 & -0.155021888 \\
\hline 14.9 & 0 & -0.000262056 & -0.173787836 & -0.156020794 \\
\hline 15 & 0 & -0.0002989 & -0.174793076 & -0.157016652 \\
\hline 15.1 & 0 & -0.000335336 & -0.175795286 & -0.158009481 \\
\hline 15.2 & 0 & -0.000371369 & -0.176794486 & -0.158999301 \\
\hline 15.3 & 0 & -0.000407 & -0.177790696 & -0.159986132 \\
\hline 15.4 & 0 & -0.000442232 & -0.178783936 & -0.160969993 \\
\hline
\end{tabular}




\begin{tabular}{|c|c|c|c|c|}
\hline 15.5 & 0 & -0.000477068 & -0.179774223 & -0.161950903 \\
\hline 15.6 & 0 & -0.000511509 & -0.180761579 & -0.162928882 \\
\hline 15.7 & 0 & -0.00054556 & -0.18174602 & -0.163903949 \\
\hline 15.8 & 0 & -0.000579222 & -0.182727567 & -0.164876121 \\
\hline 15.9 & 0 & -0.000612497 & -0.183706237 & -0.165845418 \\
\hline 16 & 0 & -0.000645389 & -0.18468205 & -0.166811858 \\
\hline 16.1 & 0 & -0.000677898 & -0.185655021 & -0.167775458 \\
\hline 16.2 & 0 & -0.000710029 & -0.186625171 & -0.168736238 \\
\hline 16.3 & 0 & -0.000741783 & -0.187592516 & -0.169694213 \\
\hline 16.4 & 0 & -0.000773163 & -0.188557073 & -0.170649403 \\
\hline 16.5 & 0 & -0.00080417 & -0.189518861 & -0.171601823 \\
\hline 16.6 & 0 & -0.000834808 & -0.190477896 & -0.172551492 \\
\hline 16.7 & 0 & -0.000865078 & -0.191434195 & -0.173498426 \\
\hline 16.8 & 0 & -0.000894982 & -0.192387776 & -0.174442642 \\
\hline 16.9 & 0 & -0.000924524 & -0.193338654 & -0.175384157 \\
\hline 17 & 0 & -0.000953705 & -0.194286846 & -0.176322987 \\
\hline 17.1 & 0 & -0.000982526 & -0.195232368 & -0.177259148 \\
\hline 17.2 & 0 & -0.001010991 & -0.196175237 & -0.178192657 \\
\hline 17.3 & 0 & -0.001039102 & -0.197115469 & -0.17912353 \\
\hline 17.4 & 0 & -0.00106686 & -0.198053079 & -0.180051782 \\
\hline 17.5 & 0 & -0.001094268 & -0.198988082 & -0.180977428 \\
\hline 17.6 & 0 & -0.001121328 & -0.199920495 & -0.181900486 \\
\hline 17.7 & 0 & -0.001148041 & -0.200850333 & -0.182820969 \\
\hline 17.8 & 0 & -0.00117441 & -0.201777611 & -0.183738893 \\
\hline 17.9 & 0 & -0.001200437 & -0.202702344 & -0.184654273 \\
\hline 18 & 0 & -0.001226124 & -0.203624547 & -0.185567124 \\
\hline 18.1 & 0 & -0.001251473 & -0.204544234 & -0.186477461 \\
\hline 18.2 & 0 & -0.001276485 & -0.205461421 & -0.187385299 \\
\hline 18.3 & 0 & -0.001301163 & -0.206376121 & -0.188290651 \\
\hline 18.4 & 0 & -0.001325509 & -0.20728835 & -0.189193532 \\
\hline 18.5 & 0 & -0.001349524 & -0.208198121 & -0.190093957 \\
\hline 18.6 & 0 & -0.00137321 & -0.209105448 & -0.190991939 \\
\hline
\end{tabular}




$\begin{array}{lllll}18.7 & 0 & -0.00139657 & -0.210010346 & -0.191887493 \\ 18.8 & 0 & -0.001419604 & -0.210912828 & -0.192780632 \\ 18.9 & 0 & -0.001442316 & -0.211812907 & -0.19367137 \\ 19 & 0 & -0.001464705 & -0.212710598 & -0.19455972 \\ 19.1 & 0 & -0.001486776 & -0.213605914 & -0.195445696 \\ 19.2 & 0 & -0.001508529 & -0.214498869 & -0.196329312 \\ 19.3 & 0 & -0.001529965 & -0.215389474 & -0.19721058 \\ 19.4 & 0 & -0.001551087 & -0.216277744 & -0.198089514 \\ 19.5 & 0 & -0.001571897 & -0.217163691 & -0.198966125 \\ 19.6 & 0 & -0.001592395 & -0.218047328 & -0.199840429 \\ 19.7 & 0 & -0.001612585 & -0.218928668 & -0.200712436 \\ 19.8 & 0 & -0.001632467 & -0.219807724 & -0.20158216 \\ 19.9 & 0 & -0.001652043 & -0.220684508 & -0.202449613 \\ 20 & 0 & -0.001671315 & -0.221559032 & -0.203314808\end{array}$

The Gibbs free energy difference of the four polytypes of boron nitride (r-BN, h-BN, c-BN, and w-BN) as a function of pressure ranging from 0 to $20 \mathrm{GPa}$, calculated with respect to $\mathrm{r}-\mathrm{BN}$ as a reference at a given temperature of 900 Kelvin. The unit of the Gibbs free energy difference for the four polytypes of boron nitride is given in $\mathrm{eV} /$ atom.

$\begin{array}{lllll}\mathbf{P}(\mathbf{G P a}) \mathbf{r}-\mathbf{B N} & \mathbf{h}-\mathbf{B N} & \mathbf{c}-\mathbf{B N} & \mathbf{w}-\mathbf{B N} \\ 0 & 0 & 0.013728465 & 0.036206699 & 0.052424295 \\ 0.1 & 0 & 0.013547905 & 0.034139229 & 0.050366334 \\ 0.2 & 0 & 0.013368972 & 0.032091622 & 0.048328236 \\ 0.3 & 0 & 0.013191697 & 0.03006324 & 0.046309363 \\ 0.4 & 0 & 0.013016103 & 0.028053487 & 0.044309118 \\ 0.5 & 0 & 0.012842203 & 0.026061803 & 0.042326944 \\ 0.6 & 0 & 0.012670005 & 0.024087663 & 0.040362312 \\ 0.7 & 0 & 0.012499512 & 0.02213057 & 0.038414728 \\ 0.8 & 0 & 0.012330721 & 0.020190056 & 0.036483722 \\ 0.9 & 0 & 0.012163628 & 0.018265679 & 0.034568853 \\ 1 & 0 & 0.011998226 & 0.016357018 & 0.032669701 \\ 1.1 & 0 & 0.011834502 & 0.014463677 & 0.030785867\end{array}$




\begin{tabular}{|c|c|c|c|c|}
\hline 1.2 & 0 & 0.011672447 & 0.012585275 & 0.028916974 \\
\hline 1.3 & 0 & 0.011512045 & 0.010721455 & 0.027062661 \\
\hline 1.4 & 0 & 0.011353284 & 0.008871871 & 0.025222585 \\
\hline 1.5 & 0 & 0.011196146 & 0.007036198 & 0.023396419 \\
\hline 1.6 & 0 & 0.011040615 & 0.005214121 & 0.021583849 \\
\hline 1.7 & 0 & 0.010886676 & 0.003405343 & 0.019784578 \\
\hline 1.8 & 0 & 0.010734309 & 0.001609576 & 0.017998317 \\
\hline 1.9 & 0 & 0.010583498 & -0.000173453 & 0.016224794 \\
\hline 2 & 0 & 0.010434224 & -0.001944009 & 0.014463744 \\
\hline 2.1 & 0 & 0.01028647 & -0.003702344 & 0.012714916 \\
\hline 2.2 & 0 & 0.010140217 & -0.0054487 & 0.010978064 \\
\hline 2.3 & 0 & 0.009995447 & -0.007183312 & 0.009252958 \\
\hline 2.4 & 0 & 0.009852143 & -0.008906405 & 0.00753937 \\
\hline 2.5 & 0 & 0.009710285 & -0.010618195 & 0.005837085 \\
\hline 2.6 & 0 & 0.009569857 & -0.012318891 & 0.004145893 \\
\hline 2.7 & 0 & 0.009430841 & -0.014008695 & 0.002465593 \\
\hline 2.8 & 0 & 0.009293218 & -0.015687801 & 0.000795991 \\
\hline 2.9 & 0 & 0.009156973 & -0.017356398 & -0.000863102 \\
\hline 3 & 0 & 0.009022086 & -0.019014667 & -0.002511868 \\
\hline 3.1 & 0 & 0.008888543 & -0.020662784 & -0.004150482 \\
\hline 3.2 & 0 & 0.008756325 & -0.022300919 & -0.005779115 \\
\hline 3.3 & 0 & 0.008625417 & -0.023929237 & -0.00739793 \\
\hline 3.4 & 0 & 0.008495803 & -0.025547897 & -0.009007089 \\
\hline 3.5 & 0 & 0.008367465 & -0.027157055 & -0.010606746 \\
\hline 3.6 & 0 & 0.00824039 & -0.028756861 & -0.01219705 \\
\hline 3.7 & 0 & 0.00811456 & -0.030347459 & -0.013778148 \\
\hline 3.8 & 0 & 0.007989962 & -0.031928992 & -0.015350181 \\
\hline 3.9 & 0 & 0.00786658 & -0.033501597 & -0.016913286 \\
\hline 4 & 0 & 0.007744399 & -0.035065407 & -0.018467597 \\
\hline 4.1 & 0 & 0.007623405 & -0.036620553 & -0.020013243 \\
\hline 4.2 & 0 & 0.007503584 & -0.03816716 & -0.021550351 \\
\hline 42 & 0 & 0.007384922 & -0.039705351 & -0.023079044 \\
\hline
\end{tabular}




\begin{tabular}{|c|c|c|c|c|}
\hline .4 & 0 & 0.007267405 & -0.041235246 & -0.024599441 \\
\hline 4.5 & 0 & 0.00715102 & -0.042756961 & -0.026111659 \\
\hline 4.6 & 0 & 0.007035754 & -0.044270609 & -0.02761581 \\
\hline 4.7 & 0 & 0.006921593 & -0.045776301 & -0.029112006 \\
\hline 4.8 & 0 & 0.006808525 & -0.047274144 & -0.030600353 \\
\hline 4.9 & 0 & 0.006696537 & -0.048764244 & -0.032080958 \\
\hline 5 & 0 & 0.006585617 & -0.050246704 & -0.033553922 \\
\hline 5.1 & 0 & 0.006475753 & -0.051721621 & -0.035019345 \\
\hline 5.2 & 0 & 0.006366933 & -0.053189095 & -0.036477325 \\
\hline 5.3 & 0 & 0.006259145 & -0.054649221 & -0.037927957 \\
\hline 5.4 & 0 & 0.006152379 & -0.056102091 & -0.039371334 \\
\hline 5.5 & 0 & 0.006046622 & -0.057547797 & -0.040807547 \\
\hline 5.6 & 0 & 0.005941863 & -0.058986426 & -0.042236685 \\
\hline 5.7 & 0 & 0.005838093 & -0.060418067 & -0.043658834 \\
\hline 5.8 & 0 & 0.005735299 & -0.061842803 & -0.045074079 \\
\hline 5.9 & 0 & 0.005633473 & -0.063260718 & -0.046482503 \\
\hline 6 & 0 & 0.005532602 & -0.064671892 & -0.047884188 \\
\hline 6.1 & 0 & 0.005432678 & -0.066076405 & -0.049279212 \\
\hline 6.2 & 0 & 0.00533369 & -0.067474336 & -0.050667653 \\
\hline 6.3 & 0 & 0.005235628 & -0.068865759 & -0.052049588 \\
\hline 6.4 & 0 & 0.005138483 & -0.070250749 & -0.053425091 \\
\hline 6.5 & 0 & 0.005042246 & -0.07162938 & -0.054794235 \\
\hline 6.6 & 0 & 0.004946907 & -0.073001723 & -0.056157091 \\
\hline 6.7 & 0 & 0.004852456 & -0.074367847 & -0.057513729 \\
\hline 6.8 & 0 & 0.004758886 & -0.075727821 & -0.058864218 \\
\hline 6.9 & 0 & 0.004666187 & -0.077081713 & -0.060208626 \\
\hline 7 & 0 & 0.00457435 & -0.078429589 & -0.061547017 \\
\hline 7.1 & 0 & 0.004483367 & -0.079771512 & -0.062879457 \\
\hline 7.2 & 0 & 0.00439323 & -0.081107547 & -0.064206009 \\
\hline 7.3 & 0 & 0.00430393 & -0.082437755 & -0.065526735 \\
\hline 7.4 & 0 & 0.004215459 & -0.083762198 & -0.066841697 \\
\hline 7.5 & 0 & 0.004127809 & -0.085080936 & -0.068150954 \\
\hline
\end{tabular}




\begin{tabular}{|c|c|c|c|c|}
\hline 7.6 & 0 & 0.004040973 & -0.086394027 & -0.069454564 \\
\hline 7.7 & 0 & 0.003954942 & -0.087701529 & -0.070752587 \\
\hline 7.8 & 0 & 0.003869709 & -0.089003498 & -0.072045077 \\
\hline 7.9 & 0 & 0.003785266 & -0.090299991 & -0.073332092 \\
\hline 8 & 0 & 0.003701606 & -0.091591062 & -0.074613685 \\
\hline 8.1 & 0 & 0.003618722 & -0.092876764 & -0.075889911 \\
\hline 8.2 & 0 & 0.003536606 & -0.09415715 & -0.077160821 \\
\hline 8.3 & 0 & 0.003455252 & -0.095432273 & -0.078426468 \\
\hline 8.4 & 0 & 0.003374653 & -0.096702183 & -0.079686903 \\
\hline 8.5 & 0 & 0.003294801 & -0.097966929 & -0.080942175 \\
\hline 8.6 & 0 & 0.00321569 & -0.099226562 & -0.082192334 \\
\hline 8.7 & 0 & 0.003137314 & -0.100481129 & -0.083437429 \\
\hline 8.8 & 0 & 0.003059665 & -0.101730679 & -0.084677507 \\
\hline 8.9 & 0 & 0.002982738 & -0.102975257 & -0.085912614 \\
\hline 9 & 0 & 0.002906525 & -0.10421491 & -0.087142797 \\
\hline 9.1 & 0 & 0.002831022 & -0.105449684 & -0.088368101 \\
\hline 9.2 & 0 & 0.002756221 & -0.106679622 & -0.08958857 \\
\hline 9.3 & 0 & 0.002682117 & -0.107904769 & -0.090804249 \\
\hline 9.4 & 0 & 0.002608703 & -0.109125168 & -0.09201518 \\
\hline 9.5 & 0 & 0.002535974 & -0.110340861 & -0.093221406 \\
\hline 9.6 & 0 & 0.002463924 & -0.11155189 & -0.094422969 \\
\hline 9.7 & 0 & 0.002392547 & -0.112758296 & -0.09561991 \\
\hline 9.8 & 0 & 0.002321838 & -0.113960119 & -0.096812269 \\
\hline 9.9 & 0 & 0.002251791 & -0.115157401 & -0.098000087 \\
\hline 10 & 0 & 0.0021824 & -0.116350179 & -0.099183402 \\
\hline 10.1 & 0 & 0.00211366 & -0.117538492 & -0.100362254 \\
\hline 10.2 & 0 & 0.002045566 & -0.118722379 & -0.10153668 \\
\hline 10.3 & 0 & 0.001978112 & -0.119901878 & -0.102706718 \\
\hline 10.4 & 0 & 0.001911294 & -0.121077025 & -0.103872405 \\
\hline 10.5 & 0 & 0.001845106 & -0.122247856 & -0.105033778 \\
\hline 10.6 & 0 & 0.001779543 & -0.123414408 & -0.106190872 \\
\hline 10.7 & 0 & 0.0017146 & -0.124576716 & -0.107343723 \\
\hline
\end{tabular}




\begin{tabular}{|c|c|c|c|c|}
\hline 10.8 & 0 & 0.001650273 & -0.125734814 & -0.108492365 \\
\hline 10.9 & 0 & 0.001586556 & -0.126888739 & -0.109636834 \\
\hline 11 & 0 & 0.001523444 & -0.128038523 & -0.110777163 \\
\hline 11.1 & 0 & 0.001460933 & -0.129184199 & -0.111913386 \\
\hline 11.2 & 0 & 0.001399019 & -0.130325801 & -0.113045535 \\
\hline 11.3 & 0 & 0.001337696 & -0.131463361 & -0.114173643 \\
\hline 11.4 & 0 & 0.00127696 & -0.132596912 & -0.115297743 \\
\hline 11.5 & 0 & 0.001216807 & -0.133726485 & -0.116417866 \\
\hline 11.6 & 0 & 0.001157231 & -0.134852111 & -0.117534043 \\
\hline 11.7 & 0 & 0.00109823 & -0.135973821 & -0.118646304 \\
\hline 11.8 & 0 & 0.001039798 & -0.137091646 & -0.119754681 \\
\hline 11.9 & 0 & 0.000981932 & -0.138205615 & -0.120859204 \\
\hline 12 & 0 & 0.000924626 & -0.139315758 & -0.121959901 \\
\hline 12.1 & 0 & 0.000867878 & -0.140422104 & -0.123056803 \\
\hline 12.2 & 0 & 0.000811682 & -0.141524683 & -0.124149937 \\
\hline 12.3 & 0 & 0.000756035 & -0.142623522 & -0.125239333 \\
\hline 12.4 & 0 & 0.000700932 & -0.143718649 & -0.126325018 \\
\hline 12.5 & 0 & 0.000646371 & -0.144810093 & -0.127407021 \\
\hline 12.6 & 0 & 0.000592346 & -0.145897881 & -0.128485369 \\
\hline 12.7 & 0 & 0.000538854 & -0.146982039 & -0.129560088 \\
\hline 12.8 & 0 & 0.000485892 & -0.148062595 & -0.130631205 \\
\hline 12.9 & 0 & 0.000433455 & -0.149139575 & -0.131698748 \\
\hline 13 & 0 & 0.000381539 & -0.150213004 & -0.132762741 \\
\hline 13.1 & 0 & 0.000330142 & -0.151282909 & -0.13382321 \\
\hline 13.2 & 0 & 0.00027926 & -0.152349315 & -0.134880182 \\
\hline 13.3 & 0 & 0.000228888 & -0.153412247 & -0.13593368 \\
\hline 13.4 & 0 & 0.000179023 & -0.15447173 & -0.13698373 \\
\hline 13.5 & 0 & 0.000129662 & -0.155527788 & -0.138030357 \\
\hline 13.6 & 0 & 0.0000808018 & -0.156580446 & -0.139073584 \\
\hline 13.7 & 0 & 0.0000324381 & -0.157629727 & -0.140113435 \\
\hline 13.8 & 0 & -0.0000154319 & -0.158675655 & -0.141149934 \\
\hline 13.9 & 0 & -0.0000628117 & -0.159718253 & -0.142183105 \\
\hline
\end{tabular}




\begin{tabular}{|c|c|c|c|c|}
\hline 14 & 0 & -0.000109705 & -0.160757545 & -0.14321297 \\
\hline 14.1 & 0 & -0.000156114 & -0.161793553 & -0.144239552 \\
\hline 14.2 & 0 & -0.000202043 & -0.162826299 & -0.145262874 \\
\hline 14.3 & 0 & -0.000247495 & -0.163855807 & -0.146282958 \\
\hline 14.4 & 0 & -0.000292472 & -0.164882098 & -0.147299826 \\
\hline 14.5 & 0 & -0.000336979 & -0.165905193 & -0.1483135 \\
\hline 14.6 & 0 & -0.000381019 & -0.166925115 & -0.149324001 \\
\hline 14.7 & 0 & -0.000424593 & -0.167941884 & -0.15033135 \\
\hline 14.8 & 0 & -0.000467706 & -0.168955523 & -0.15133557 \\
\hline 14.9 & 0 & -0.000510359 & -0.16996605 & -0.15233668 \\
\hline 15 & 0 & -0.000552557 & -0.170973488 & -0.153334701 \\
\hline 15.1 & 0 & -0.000594303 & -0.171977855 & -0.154329653 \\
\hline 15.2 & 0 & -0.000635598 & -0.172979174 & -0.155321557 \\
\hline 15.3 & 0 & -0.000676446 & -0.173977463 & -0.156310432 \\
\hline 15.4 & 0 & -0.00071685 & -0.174972741 & -0.157296299 \\
\hline 15.5 & 0 & -0.000756813 & -0.17596503 & -0.158279176 \\
\hline 15.6 & 0 & -0.000796337 & -0.176954347 & -0.159259083 \\
\hline 15.7 & 0 & -0.000835424 & -0.177940712 & -0.160236039 \\
\hline 15.8 & 0 & -0.000874079 & -0.178924144 & -0.161210063 \\
\hline 15.9 & 0 & -0.000912303 & -0.179904661 & -0.162181173 \\
\hline 16 & 0 & -0.0009501 & -0.180882282 & -0.163149388 \\
\hline 16.1 & 0 & -0.000987471 & -0.181857025 & -0.164114727 \\
\hline 16.2 & 0 & -0.001024419 & -0.182828908 & -0.165077206 \\
\hline 16.3 & 0 & -0.001060947 & -0.18379795 & -0.166036845 \\
\hline 16.4 & 0 & -0.001097058 & -0.184764167 & -0.166993661 \\
\hline 16.5 & 0 & -0.001132754 & -0.185727577 & -0.167947671 \\
\hline 16.6 & 0 & -0.001168038 & -0.186688198 & -0.168898893 \\
\hline 16.7 & 0 & -0.001202911 & -0.187646047 & -0.169847343 \\
\hline 16.8 & 0 & -0.001237377 & -0.188601141 & -0.17079304 \\
\hline 16.9 & 0 & -0.001271437 & -0.189553496 & -0.171735999 \\
\hline 17 & 0 & -0.001305095 & -0.19050313 & -0.172676238 \\
\hline 17.1 & 0 & -0.001338353 & -0.191450058 & -0.173613772 \\
\hline
\end{tabular}




$\begin{array}{lllll}17.2 & 0 & -0.001371213 & -0.192394297 & -0.174548619 \\ 17.3 & 0 & -0.001403677 & -0.193335863 & -0.175480794 \\ 17.4 & 0 & -0.001435748 & -0.194274773 & -0.176410313 \\ 17.5 & 0 & -0.001467427 & -0.195211042 & -0.177337193 \\ 17.6 & 0 & -0.001498718 & -0.196144685 & -0.178261448 \\ 17.7 & 0 & -0.001529622 & -0.197075718 & -0.179183095 \\ 17.8 & 0 & -0.001560142 & -0.198004157 & -0.180102148 \\ 17.9 & 0 & -0.00159028 & -0.198930017 & -0.181018624 \\ 18 & 0 & -0.001620038 & -0.199853313 & -0.181932536 \\ 18.1 & 0 & -0.001649418 & -0.20077406 & -0.182843901 \\ 18.2 & 0 & -0.001678422 & -0.201692273 & -0.183752732 \\ 18.3 & 0 & -0.001707053 & -0.202607965 & -0.184659045 \\ 18.4 & 0 & -0.001735312 & -0.203521153 & -0.185562854 \\ 18.5 & 0 & -0.001763202 & -0.20443185 & -0.186464173 \\ 18.6 & 0 & -0.001790725 & -0.20534007 & -0.187363017 \\ 18.7 & 0 & -0.001817882 & -0.206245828 & -0.1882594 \\ 18.8 & 0 & -0.001844677 & -0.207149137 & -0.189153336 \\ 18.9 & 0 & -0.00187111 & -0.208050012 & -0.190044838 \\ 19 & 0 & -0.001897183 & -0.208948466 & -0.19093392 \\ 19.1 & 0 & -0.0019229 & -0.209844512 & -0.191820596 \\ 19.2 & 0 & -0.001948261 & -0.210738165 & -0.19270488 \\ 19.3 & 0 & -0.001973268 & -0.211629438 & -0.193586785 \\ 19.4 & 0 & -0.001997924 & -0.212518343 & -0.194466323 \\ 19.5 & 0 & -0.002022231 & -0.213404894 & -0.195343509 \\ 19.6 & 0 & -0.002046189 & -0.214289104 & -0.196218355 \\ 19.9 & 0 & -0.002069802 & -0.215170986 & -0.197090873 \\ 19 & -0.002093071 & -0.216050552 & -0.197961078 \\ 19.0002115998 & -0.216927816 & -0.198828981 \\ 10.002138584 & -0.217802789 & -0.199694595\end{array}$


The Gibbs free energy difference of the four polytypes of boron nitride ( $\mathrm{r}-\mathrm{BN}, \mathrm{h}-\mathrm{BN}, \mathrm{c}-\mathrm{BN}$, and w-BN) as a function of pressure ranging from 0 to $20 \mathrm{GPa}$, calculated with respect to $\mathrm{r}-\mathrm{BN}$ as a reference at a given temperature of $\mathbf{1 0 0 0}$ Kelvin. The unit of the Gibbs free energy difference for the four polytypes of boron nitride is given in $\mathrm{eV} / \mathrm{atom}$.

\begin{tabular}{lllll}
$\mathbf{P}(\mathbf{G P a}) \mathbf{r}-\mathbf{B N}$ & $\mathbf{h}-\mathbf{B N}$ & $\mathbf{c}-\mathbf{B N}$ & $\mathbf{w}-\mathbf{B N}$ \\
0 & 0 & 0.01497464 & 0.040872936 & 0.05695879 \\
0.1 & 0 & 0.014781224 & 0.038797337 & 0.054892642 \\
0.2 & 0 & 0.014589233 & 0.036741397 & 0.052846153 \\
0.3 & 0 & 0.014398726 & 0.034704507 & 0.050818714 \\
0.4 & 0 & 0.014209753 & 0.032686096 & 0.048809753 \\
0.5 & 0 & 0.01402235 & 0.030685627 & 0.046818734 \\
0.6 & 0 & 0.013836545 & 0.028702593 & 0.044845149 \\
0.7 & 0 & 0.013652359 & 0.026736515 & 0.042888521 \\
0.8 & 0 & 0.013469806 & 0.024786941 & 0.040948397 \\
0.9 & 0 & 0.013288897 & 0.022853442 & 0.039024347 \\
1 & 0 & 0.013109636 & 0.020935612 & 0.037115965 \\
1.1 & 0 & 0.012932024 & 0.019033063 & 0.035222865 \\
1.2 & 0 & 0.01275606 & 0.017145426 & 0.033344677 \\
1.3 & 0 & 0.01258174 & 0.015272352 & 0.03148105 \\
1.4 & 0 & 0.012409058 & 0.013413505 & 0.029631651 \\
1.5 & 0 & 0.012238006 & 0.011568565 & 0.027796158 \\
1.6 & 0 & 0.012068574 & 0.009737226 & 0.025974267 \\
1.7 & 0 & 0.011900752 & 0.007919195 & 0.024165683 \\
1.8 & 0 & 0.011734527 & 0.006114191 & 0.022370125 \\
1.9 & 0 & 0.011569888 & 0.004321945 & 0.020587325 \\
2.5 & 0 & 0.011406821 & 0.002542198 & 0.018817024 \\
2.1 & 0 & 0.011245312 & 0.000774701 & 0.017058973 \\
2.2 & 0 & 0.011085347 & -0.000980785 & 0.015312932 \\
2.3 & 0 & 0.010926912 & -0.002724491 & 0.013578672 \\
\hline .4 & 0 & 0.010769991 & -0.004456637 & 0.01185597 \\
\hline & 0 & 0.010614569 & -0.006177439 & 0.010144612 \\
0.010460631 & -0.007887102 & 0.008444393
\end{tabular}




\begin{tabular}{|c|c|c|c|c|}
\hline 2.7 & 0 & 0.010308162 & -0.009585826 & 0.006755113 \\
\hline 2.8 & 0 & 0.010157146 & -0.011273803 & 0.005076579 \\
\hline 2.9 & 0 & 0.010007568 & -0.012951219 & 0.003408605 \\
\hline 3 & 0 & 0.009859412 & -0.014618256 & 0.001751011 \\
\hline 3.1 & 0 & 0.009712664 & -0.016275085 & 0.000103624 \\
\hline 3.2 & 0 & 0.009567308 & -0.017921877 & -0.001533727 \\
\hline 3.3 & 0 & 0.009423328 & -0.019558795 & -0.003161204 \\
\hline 3.4 & 0 & 0.00928071 & -0.021185998 & -0.004778965 \\
\hline 3.5 & 0 & 0.009139439 & -0.022803638 & -0.006387165 \\
\hline 3.6 & 0 & 0.0089995 & -0.024411866 & -0.007985953 \\
\hline 3.7 & 0 & 0.008860879 & -0.026010826 & -0.009575474 \\
\hline 3.8 & 0 & 0.00872356 & -0.027600658 & -0.011155868 \\
\hline 3.9 & 0 & 0.008587531 & -0.029181501 & -0.012727271 \\
\hline 4 & 0 & 0.008452776 & -0.030753485 & -0.014289818 \\
\hline 4.1 & 0 & 0.008319281 & -0.032316742 & -0.015843636 \\
\hline 4.2 & 0 & 0.008187034 & -0.033871396 & -0.017388853 \\
\hline 4.3 & 0 & 0.00805602 & -0.035417569 & -0.01892559 \\
\hline 4.4 & 0 & 0.007926226 & -0.036955382 & -0.020453966 \\
\hline 4.5 & 0 & 0.007797639 & -0.03848495 & -0.021974098 \\
\hline 4.6 & 0 & 0.007670246 & -0.040006386 & -0.023486099 \\
\hline 4.7 & 0 & 0.007544034 & -0.0415198 & -0.024990079 \\
\hline 4.8 & 0 & 0.00741899 & -0.043025301 & -0.026486145 \\
\hline 4.9 & 0 & 0.007295103 & -0.044522992 & -0.027974403 \\
\hline 5 & 0 & 0.007172359 & -0.046012977 & -0.029454954 \\
\hline 5.1 & 0 & 0.007050747 & -0.047495354 & -0.030927899 \\
\hline 5.2 & 0 & 0.006930255 & -0.048970222 & -0.032393335 \\
\hline 5.3 & 0 & 0.00681087 & -0.050437676 & -0.033851357 \\
\hline 5.4 & 0 & 0.006692583 & -0.051897809 & -0.035302058 \\
\hline 5.5 & 0 & 0.006575381 & -0.053350711 & -0.03674553 \\
\hline 5.6 & 0 & 0.006459253 & -0.054796472 & -0.03818186 \\
\hline 5.7 & 0 & 0.006344188 & -0.056235178 & -0.039611137 \\
\hline 5.8 & 0 & 0.006230175 & -0.057666915 & -0.041033444 \\
\hline
\end{tabular}




\begin{tabular}{|c|c|c|c|c|}
\hline 5.9 & 0 & 0.006117204 & -0.059091765 & -0.042448866 \\
\hline 6 & 0 & 0.006005265 & -0.060509809 & -0.043857482 \\
\hline 6.1 & 0 & 0.005894346 & -0.061921128 & -0.045259374 \\
\hline 6.2 & 0 & 0.005784437 & -0.063325798 & -0.046654618 \\
\hline 6.3 & 0 & 0.00567553 & -0.064723898 & -0.048043291 \\
\hline 6.4 & 0 & 0.005567613 & -0.0661155 & -0.049425467 \\
\hline 6.5 & 0 & 0.005460678 & -0.067500678 & -0.050801221 \\
\hline 6.6 & 0 & 0.005354714 & -0.068879504 & -0.052170622 \\
\hline 6.7 & 0 & 0.005249712 & -0.070252048 & -0.053533743 \\
\hline 6.8 & 0 & 0.005145663 & -0.071618379 & -0.054890651 \\
\hline 6.9 & 0 & 0.005042558 & -0.072978565 & -0.056241414 \\
\hline 7 & 0 & 0.004940388 & -0.074332671 & -0.057586098 \\
\hline 7.1 & 0 & 0.004839144 & -0.075680762 & -0.058924768 \\
\hline 7.2 & 0 & 0.004738817 & -0.077022903 & -0.060257488 \\
\hline 7.3 & 0 & 0.004639399 & -0.078359155 & -0.06158432 \\
\hline 7.4 & 0 & 0.004540881 & -0.07968958 & -0.062905325 \\
\hline 7.5 & 0 & 0.004443255 & -0.081014238 & -0.064220565 \\
\hline 7.6 & 0 & 0.004346513 & -0.082333189 & -0.065530097 \\
\hline 7.7 & 0 & 0.004250646 & -0.083646489 & -0.06683398 \\
\hline 7.8 & 0 & 0.004155648 & -0.084954196 & -0.06813227 \\
\hline 7.9 & 0 & 0.004061509 & -0.086256366 & -0.069425024 \\
\hline 8 & 0 & 0.003968222 & -0.087553055 & -0.070712297 \\
\hline 8.1 & 0 & 0.00387578 & -0.088844315 & -0.071994142 \\
\hline 8.2 & 0 & 0.003784175 & -0.090130199 & -0.073270613 \\
\hline 8.3 & 0 & 0.0036934 & -0.091410761 & -0.074541761 \\
\hline 8.4 & 0 & 0.003603447 & -0.092686051 & -0.075807639 \\
\hline 8.5 & 0 & 0.003514309 & -0.09395612 & -0.077068295 \\
\hline 8.6 & 0 & 0.003425979 & -0.095221017 & -0.078323781 \\
\hline 8.7 & 0 & 0.003338451 & -0.09648079 & -0.079574143 \\
\hline 8.8 & 0 & 0.003251717 & -0.097735488 & -0.080819431 \\
\hline 8.9 & 0 & 0.00316577 & -0.098985158 & -0.082059692 \\
\hline 9 & 0 & 0.003080605 & -0.100229846 & -0.083294972 \\
\hline
\end{tabular}




\begin{tabular}{|c|c|c|c|c|}
\hline .1 & 0 & 0.002996213 & -0.101469598 & -0.084525315 \\
\hline 9.2 & 0 & 0.00291259 & -0.102704458 & -0.085750768 \\
\hline 9.3 & 0 & 0.002829727 & -0.10393447 & -0.086971375 \\
\hline 9.4 & & 0.00274762 & -0.105159679 & -0.088187178 \\
\hline 9.5 & 0 & 0.002666261 & -0.106380127 & -0.089398221 \\
\hline 9.6 & 0 & 0.002585646 & -0.107595856 & -0.090604545 \\
\hline 9.7 & 0 & 0.002505766 & -0.108806907 & -0.091806193 \\
\hline 9.8 & 0 & 0.002426618 & -0.110013321 & -0.093003205 \\
\hline 9.9 & 0 & 0.002348194 & -0.111215139 & -0.094195621 \\
\hline 10 & 0 & 0.002270489 & -0.1124124 & -0.095383481 \\
\hline 10.1 & 0 & 0.002193497 & -0.113605143 & -0.096566823 \\
\hline 10.2 & 0 & 0.002117212 & -0.114793406 & -0.097745687 \\
\hline 10.3 & 0 & 0.00204163 & -0.115977228 & -0.09892011 \\
\hline 10.4 & 0 & 0.001966744 & -0.117156646 & -0.10009013 \\
\hline 10.5 & 0 & 0.001892549 & -0.118331695 & -0.101255783 \\
\hline 10.6 & 0 & 0.001819039 & -0.119502414 & -0.102417105 \\
\hline 10.7 & 0 & 0.00174621 & -0.120668837 & -0.103574132 \\
\hline 10.8 & 0 & 0.001674055 & -0.121830999 & -0.1047269 \\
\hline 10.9 & 0 & 0.001602571 & -0.122988936 & -0.105875443 \\
\hline 11 & 0 & 0.001531751 & -0.124142682 & -0.107019795 \\
\hline 11.1 & 0 & 0.001461591 & -0.125292269 & -0.10815999 \\
\hline 11.2 & 0 & 0.001392085 & -0.126437733 & -0.109296062 \\
\hline 11.3 & 0 & 0.00132323 & -0.127579104 & -0.110428043 \\
\hline 11.4 & 0 & 0.001255019 & -0.128716417 & -0.111555965 \\
\hline 11.5 & 0 & 0.001187448 & -0.129849702 & -0.112679861 \\
\hline 11.6 & 0 & 0.001120513 & -0.130978991 & -0.113799762 \\
\hline 11.7 & 0 & 0.001054209 & -0.132104316 & -0.1149157 \\
\hline 11.8 & 0 & 0.00098853 & -0.133225707 & -0.116027704 \\
\hline 11.9 & 0 & 0.000923474 & -0.134343194 & -0.117135805 \\
\hline 12 & 0 & 0.000859034 & -0.135456807 & -0.118240033 \\
\hline 12.1 & 0 & 0.000795207 & -0.136566576 & -0.119340418 \\
\hline 12.2 & 0 & 0.000731988 & -0.137672529 & -0.120436988 \\
\hline
\end{tabular}




\begin{tabular}{|c|c|c|c|c|}
\hline 2.3 & 0 & 0.000669373 & -0.138774696 & -0.121529773 \\
\hline 12.4 & 0 & 0.000607357 & -0.139873105 & -0.1226188 \\
\hline 12.5 & 0 & 0.000545937 & -0.140967783 & -0.123704098 \\
\hline 12.6 & 0 & 0.000485108 & -0.142058759 & -0.124785694 \\
\hline 12.7 & 0 & 0.000424866 & -0.14314606 & -0.125863616 \\
\hline 12.8 & 0 & 0.000365207 & -0.144229713 & -0.126937891 \\
\hline 12.9 & 0 & 0.000306127 & -0.145309743 & -0.128008545 \\
\hline 13 & 0 & 0.000247621 & -0.146386179 & -0.129075604 \\
\hline 13.1 & 0 & 0.000189687 & -0.147459045 & -0.130139094 \\
\hline 13.2 & 0 & 0.000132319 & -0.148528367 & -0.131199042 \\
\hline 13.3 & 0 & 0.0000755146 & -0.149594171 & -0.132255472 \\
\hline 13.4 & 0 & 0.0000192693 & -0.150656481 & -0.13330841 \\
\hline 13.5 & 0 & -0.0000364204 & -0.151715322 & -0.13435788 \\
\hline 13.6 & 0 & -0.0000915582 & -0.15277072 & -0.135403906 \\
\hline 13.7 & 0 & -0.000146148 & -0.153822697 & -0.136446514 \\
\hline 13.8 & 0 & -0.000200193 & -0.154871278 & -0.137485726 \\
\hline 13.9 & 0 & -0.000253698 & -0.155916487 & -0.138521566 \\
\hline 14 & 0 & -0.000306665 & -0.156958346 & -0.139554058 \\
\hline 14.1 & 0 & -0.000359099 & -0.157996879 & -0.140583225 \\
\hline 14.2 & 0 & -0.000411002 & -0.159032108 & -0.141609089 \\
\hline 14.3 & 0 & -0.000462378 & -0.160064056 & -0.142631673 \\
\hline 14.4 & 0 & -0.000513232 & -0.161092746 & -0.143650999 \\
\hline 14.5 & 0 & -0.000563565 & -0.162118199 & -0.14466709 \\
\hline 14.6 & 0 & -0.000613382 & -0.163140437 & -0.145679966 \\
\hline 14.7 & 0 & -0.000662685 & -0.164159481 & -0.14668965 \\
\hline 14.8 & 0 & -0.000711478 & -0.165175353 & -0.147696162 \\
\hline 14.9 & 0 & -0.000759764 & -0.166188074 & -0.148699524 \\
\hline 15 & 0 & -0.000807547 & -0.167197664 & -0.149699757 \\
\hline 15.1 & 0 & -0.000854829 & -0.168204145 & -0.150696881 \\
\hline 15.2 & 0 & -0.000901614 & -0.169207536 & -0.151690916 \\
\hline 15.3 & 0 & -0.000947905 & -0.170207858 & -0.152681883 \\
\hline 15.4 & 0 & -0.000993704 & -0.17120513 & -0.153669802 \\
\hline
\end{tabular}




\begin{tabular}{|c|c|c|c|c|}
\hline 15.5 & 0 & -0.001039016 & -0.172199372 & -0.154654692 \\
\hline 15.6 & 0 & -0.001083842 & -0.173190605 & -0.155636572 \\
\hline 15.7 & 0 & -0.001128187 & -0.174178846 & -0.156615462 \\
\hline 15.8 & , & -0.001172052 & -0.175164115 & -0.157591382 \\
\hline 15.9 & 0 & -0.001215441 & -0.176146431 & -0.158564349 \\
\hline 16 & 0 & -0.001258356 & -0.177125813 & -0.159534383 \\
\hline 16.1 & 0 & -0.001300801 & -0.178102279 & -0.160501502 \\
\hline 16.2 & 0 & -0.001342778 & -0.179075847 & -0.161465724 \\
\hline 16.3 & 0 & -0.001384291 & -0.180046535 & -0.162427068 \\
\hline 16.4 & 0 & -0.001425341 & -0.181014362 & -0.163385551 \\
\hline 16.5 & 0 & -0.001465933 & -0.181979345 & -0.164341191 \\
\hline 16.6 & 0 & -0.001506067 & -0.182941501 & -0.165294005 \\
\hline 16.7 & 0 & -0.001545747 & -0.183900849 & -0.166244012 \\
\hline 16.8 & 0 & -0.001584977 & -0.184857404 & -0.167191227 \\
\hline 16.9 & 0 & -0.001623757 & -0.185811185 & -0.168135669 \\
\hline 17 & 0 & -0.001662092 & -0.186762207 & -0.169077354 \\
\hline 17.1 & 0 & -0.001699982 & -0.187710488 & -0.170016298 \\
\hline 17.2 & 0 & -0.001737433 & -0.188656045 & -0.170952519 \\
\hline 17.3 & 0 & -0.001774445 & -0.189598893 & -0.171886032 \\
\hline 17.4 & 0 & -0.001811021 & -0.190539048 & -0.172816854 \\
\hline 17.5 & 0 & -0.001847163 & -0.191476527 & -0.173745001 \\
\hline 17.6 & 0 & -0.001882875 & -0.192411346 & -0.174670488 \\
\hline 17.7 & 0 & -0.001918159 & -0.19334352 & -0.175593331 \\
\hline 17.8 & 0 & -0.001953016 & -0.194273064 & -0.176513546 \\
\hline 17.9 & 0 & -0.00198745 & -0.195199995 & -0.177431149 \\
\hline 18 & 0 & -0.002021462 & -0.196124328 & -0.178346154 \\
\hline 18.1 & 0 & -0.002055056 & -0.197046077 & -0.179258577 \\
\hline 18.2 & 0 & -0.002088233 & -0.197965257 & -0.180168432 \\
\hline 18.3 & 0 & -0.002120996 & -0.198881884 & -0.181075735 \\
\hline 18.4 & 0 & -0.002153347 & -0.199795972 & -0.1819805 \\
\hline 18.5 & 0 & -0.002185288 & -0.200707536 & -0.182882742 \\
\hline 18.6 & 0 & -0.002216822 & -0.20161659 & -0.183782475 \\
\hline
\end{tabular}




$\begin{array}{lllll}18.7 & 0 & -0.002247951 & -0.202523148 & -0.184679713 \\ 18.8 & 0 & -0.002278676 & -0.203427225 & -0.185574471 \\ 18.9 & 0 & -0.002309001 & -0.204328834 & -0.186466762 \\ 19 & 0 & -0.002338927 & -0.205227989 & -0.187356601 \\ 19.1 & 0 & -0.002368457 & -0.206124705 & -0.188244002 \\ 19.2 & 0 & -0.002397592 & -0.207018995 & -0.189128977 \\ 19.3 & 0 & -0.002426335 & -0.207910872 & -0.190011541 \\ 19.4 & 0 & -0.002454688 & -0.208800349 & -0.190891706 \\ 19.5 & 0 & -0.002482653 & -0.209687441 & -0.191769487 \\ 19.6 & 0 & -0.002510232 & -0.21057216 & -0.192644895 \\ 19.7 & 0 & -0.002537426 & -0.211454519 & -0.193517945 \\ 19.8 & 0 & -0.002564239 & -0.212334531 & -0.194388649 \\ 19.9 & 0 & -0.002590671 & -0.213212208 & -0.195257021 \\ 20 & 0 & -0.002616726 & -0.214087565 & -0.196123072\end{array}$

The Gibbs free energy difference of the four polytypes of boron nitride ( $\mathrm{r}-\mathrm{BN}, \mathrm{h}-\mathrm{BN}, \mathrm{c}-\mathrm{BN}$, and w-BN) as a function of pressure ranging from 0 to $20 \mathrm{GPa}$, calculated with respect to $\mathrm{r}-\mathrm{BN}$ as a reference at a given temperature of 1100 Kelvin. The unit of the Gibbs free energy difference for the four polytypes of boron nitride is given in $\mathrm{eV} /$ atom.

$\begin{array}{lllll}\mathbf{P}(\mathbf{G P a}) \mathbf{r}-\mathbf{B N} & \mathbf{h}-\mathbf{B N} & \mathbf{c}-\mathbf{B N} & \mathbf{w}-\mathbf{B N} \\ 0 & 0 & 0.016252031 & 0.04549095 & 0.061425277 \\ 0.1 & 0 & 0.016046147 & 0.043408136 & 0.059351928 \\ 0.2 & 0 & 0.015841459 & 0.041344737 & 0.057297995 \\ 0.3 & 0 & 0.015638057 & 0.039300173 & 0.055262898 \\ 0.4 & 0 & 0.015436016 & 0.0372739 & 0.053246092 \\ 0.5 & 0 & 0.015235396 & 0.035265405 & 0.051247063 \\ 0.6 & 0 & 0.015036248 & 0.033274201 & 0.049265326 \\ 0.7 & 0 & 0.014838611 & 0.031299829 & 0.047300422 \\ 0.8 & 0 & 0.014642517 & 0.029341854 & 0.045351914 \\ 0.9 & 0 & 0.01444799 & 0.027399863 & 0.043419391 \\ 1 & 0 & 0.014255049 & 0.025473461 & 0.041502457 \\ 1.1 & 0 & 0.014063709 & 0.023562275 & 0.039600739\end{array}$




\begin{tabular}{|c|c|c|c|c|}
\hline 1.2 & 0 & 0.013873978 & 0.021665948 & 0.03771388 \\
\hline 1.3 & 0 & 0.013685864 & 0.019784139 & 0.035841539 \\
\hline 1.4 & 0 & 0.013499368 & 0.017916521 & 0.03398339 \\
\hline 1.5 & 0 & 0.013314492 & 0.016062785 & 0.032139123 \\
\hline 1.6 & 0 & 0.013131234 & 0.014222631 & 0.030308438 \\
\hline 1.7 & 0 & 0.012949589 & 0.012395774 & 0.028491049 \\
\hline 1.8 & 0 & 0.012769554 & 0.010581938 & 0.026686683 \\
\hline 1.9 & 0 & 0.01259112 & 0.008780861 & 0.024895075 \\
\hline 2 & 0 & 0.012414279 & 0.006992289 & 0.023115972 \\
\hline 2.1 & 0 & 0.012239023 & 0.005215978 & 0.02134913 \\
\hline 2.2 & 0 & 0.012065342 & 0.003451693 & 0.019594315 \\
\hline 2.3 & 0 & 0.011893225 & 0.001699209 & 0.0178513 \\
\hline 2.4 & 0 & 0.011722661 & -0.0000416933 & 0.016119867 \\
\hline 2.5 & 0 & 0.011553639 & -0.001771224 & 0.014399806 \\
\hline 2.6 & 0 & 0.011386145 & -0.003489586 & 0.012690913 \\
\hline 2.7 & 0 & 0.011220167 & -0.005196977 & 0.010992992 \\
\hline 2.8 & 0 & 0.011055693 & -0.006893585 & 0.009305853 \\
\hline 2.9 & 0 & 0.01089271 & -0.008579594 & 0.007629313 \\
\hline 3 & 0 & 0.010731204 & -0.010255183 & 0.005963194 \\
\hline 3.1 & 0 & 0.010571161 & -0.011920523 & 0.004307323 \\
\hline 3.2 & 0 & 0.01041257 & -0.013575782 & 0.002661534 \\
\hline 3.3 & 0 & 0.010255415 & -0.01522112 & 0.001025665 \\
\hline 3.4 & 0 & 0.010099683 & -0.016856696 & -0.000600441 \\
\hline 3.5 & 0 & 0.009945361 & -0.018482661 & -0.002216937 \\
\hline 3.6 & 0 & 0.009792436 & -0.020099163 & -0.00382397 \\
\hline 3.7 & 0 & 0.009640894 & -0.021706345 & -0.005421683 \\
\hline 3.8 & 0 & 0.00949072 & -0.023304348 & -0.007010217 \\
\hline 3.9 & 0 & 0.009341903 & -0.024893307 & -0.008589707 \\
\hline 4 & 0 & 0.009194429 & -0.026473354 & -0.010160286 \\
\hline 4.1 & 0 & 0.009048284 & -0.028044617 & -0.011722081 \\
\hline 4.2 & 0 & 0.008903456 & -0.029607222 & -0.013275217 \\
\hline 4.3 & 0 & 0.008759931 & -0.03116129 & -0.014819816 \\
\hline
\end{tabular}




\begin{tabular}{|c|c|c|c|c|}
\hline & 0 & 0.008617696 & -0.032706939 & -0.016355998 \\
\hline 4.5 & 0 & 0.00847674 & -0.034244286 & -0.017883876 \\
\hline 4.6 & 0 & 0.008337049 & -0.035773442 & -0.019403565 \\
\hline 47 & & 0.008198611 & -0.037294519 & -0.020915174 \\
\hline 4.8 & 0 & 0.008061414 & -0.038807622 & -0.022418809 \\
\hline 4.9 & 0 & 0.007925445 & -0.040312856 & -0.023914577 \\
\hline 5 & 0 & 0.007790693 & -0.041810324 & -0.025402577 \\
\hline 5.1 & 0 & 0.007657145 & -0.043300124 & -0.026882911 \\
\hline 5.2 & 0 & 0.00752479 & -0.044782355 & -0.028355675 \\
\hline 5.3 & 0 & 0.007393616 & -0.046257111 & -0.029820965 \\
\hline 5.4 & 0 & 0.007263612 & -0.047724485 & -0.031278873 \\
\hline 5.5 & 0 & 0.007134767 & -0.049184568 & -0.03272949 \\
\hline 5.6 & 0 & 0.007007068 & -0.050637448 & -0.034172905 \\
\hline 5.7 & 0 & 0.006880507 & -0.052083213 & -0.035609205 \\
\hline 5.8 & 0 & 0.00675507 & -0.053521948 & -0.037038474 \\
\hline 5.9 & 0 & 0.006630749 & -0.054953734 & -0.038460796 \\
\hline 6 & 0 & 0.006507532 & -0.056378654 & -0.039876252 \\
\hline 6.1 & 0 & 0.006385409 & -0.057796788 & -0.041284921 \\
\hline 6.2 & 0 & 0.006264369 & -0.059208212 & -0.042686882 \\
\hline 6.3 & 0 & 0.006144403 & -0.060613004 & -0.044082211 \\
\hline 6.4 & 0 & 0.0060255 & -0.062011239 & -0.045470981 \\
\hline 6.5 & 0 & 0.005907651 & -0.063402988 & -0.046853268 \\
\hline 6.6 & 0 & 0.005790845 & -0.064788325 & -0.048229143 \\
\hline 6.7 & 0 & 0.005675075 & -0.066167319 & -0.049598675 \\
\hline 6.8 & 0 & 0.005560328 & -0.06754004 & -0.050961934 \\
\hline 6.9 & 0 & 0.005446598 & -0.068906555 & -0.052318988 \\
\hline 7 & 0 & 0.005333874 & -0.070266931 & -0.053669903 \\
\hline 7.1 & 0 & 0.005222147 & -0.071621232 & -0.055014743 \\
\hline 7.2 & 0 & 0.005111408 & -0.072969522 & -0.056353573 \\
\hline 7.3 & 0 & 0.005001649 & -0.074311864 & -0.057686456 \\
\hline 7.4 & 0 & 0.004892861 & -0.07564832 & -0.059013453 \\
\hline 7.5 & 0 & 0.004785035 & -0.07697895 & -0.060334624 \\
\hline
\end{tabular}




\begin{tabular}{|c|c|c|c|c|}
\hline 7.6 & 0 & 0.004678163 & -0.078303813 & -0.061650028 \\
\hline 7.7 & 0 & 0.004572236 & -0.079622967 & -0.062959724 \\
\hline 7.8 & 0 & 0.004467247 & -0.080936469 & -0.064263769 \\
\hline 7.9 & 0 & 0.004363187 & -0.082244376 & -0.065562219 \\
\hline 8 & 0 & 0.004260048 & -0.083546743 & -0.06685513 \\
\hline 8.1 & 0 & 0.004157822 & -0.084843624 & -0.068142554 \\
\hline 8.2 & 0 & 0.004056502 & -0.086135072 & -0.069424547 \\
\hline 8.3 & 0 & 0.003956079 & -0.08742114 & -0.070701159 \\
\hline 8.4 & 0 & 0.003856547 & -0.088701878 & -0.071972443 \\
\hline 8.5 & 0 & 0.003757897 & -0.089977339 & -0.073238449 \\
\hline 8.6 & 0 & 0.003660123 & -0.09124757 & -0.074499227 \\
\hline 8.7 & 0 & 0.003563217 & -0.092512622 & -0.075754826 \\
\hline 8.8 & 0 & 0.003467172 & -0.093772543 & -0.077005294 \\
\hline 8.9 & 0 & 0.003371981 & -0.095027379 & -0.078250678 \\
\hline 9 & 0 & 0.003277637 & -0.096277177 & -0.079491025 \\
\hline 9.1 & 0 & 0.003184132 & -0.097521984 & -0.080726381 \\
\hline 9.2 & 0 & 0.003091461 & -0.098761844 & -0.08195679 \\
\hline 9.3 & 0 & 0.002999616 & -0.099996802 & -0.083182298 \\
\hline 9.4 & 0 & 0.002908591 & -0.101226901 & -0.084402948 \\
\hline 9.5 & 0 & 0.00281838 & -0.102452185 & -0.085618783 \\
\hline 9.6 & 0 & 0.002728975 & -0.103672696 & -0.086829846 \\
\hline 9.7 & 0 & 0.002640371 & -0.104888475 & -0.088036178 \\
\hline 9.8 & 0 & 0.002552561 & -0.106099563 & -0.089237819 \\
\hline 9.9 & 0 & 0.002465539 & -0.107306002 & -0.090434812 \\
\hline 10 & 0 & 0.002379298 & -0.108507831 & -0.091627195 \\
\hline 10.1 & 0 & 0.002293834 & -0.109705089 & -0.092815008 \\
\hline 10.2 & 0 & 0.002209139 & -0.110897815 & -0.09399829 \\
\hline 10.3 & 0 & 0.002125209 & -0.112086048 & -0.095177078 \\
\hline 10.4 & 0 & 0.002042036 & -0.113269823 & -0.096351411 \\
\hline 10.5 & 0 & 0.001959616 & -0.11444918 & -0.097521325 \\
\hline 10.6 & 0 & 0.001877942 & -0.115624154 & -0.098686857 \\
\hline 10.7 & 0 & 0.00179701 & -0.11679478 & -0.099848042 \\
\hline
\end{tabular}




\begin{tabular}{|c|c|c|c|c|}
\hline 10.8 & 0 & 0.001716813 & -0.117961096 & -0.101004917 \\
\hline 10.9 & 0 & 0.001637346 & -0.119123135 & -0.102157517 \\
\hline 11 & 0 & 0.001558603 & -0.120280932 & -0.103305875 \\
\hline 11.1 & 0 & 0.00148058 & -0.121434521 & -0.104450025 \\
\hline 11.2 & 0 & 0.001403271 & -0.122583936 & -0.105590003 \\
\hline 11.3 & 0 & 0.001326671 & -0.12372921 & -0.106725839 \\
\hline 11.4 & 0 & 0.001250774 & -0.124870375 & -0.107857568 \\
\hline 11.5 & 0 & 0.001175576 & -0.126007463 & -0.108985221 \\
\hline 11.6 & 0 & 0.001101072 & -0.127140507 & -0.11010883 \\
\hline 11.7 & 0 & 0.001027256 & -0.128269538 & -0.111228426 \\
\hline 11.8 & 0 & 0.000954124 & -0.129394586 & -0.112344041 \\
\hline 11.9 & 0 & 0.00088167 & -0.130515683 & -0.113455705 \\
\hline 12 & 0 & 0.000809891 & -0.131632857 & -0.114563448 \\
\hline 12.1 & 0 & 0.00073878 & -0.13274614 & -0.115667299 \\
\hline 12.2 & 0 & 0.000668335 & -0.13385556 & -0.116767289 \\
\hline 12.3 & 0 & 0.000598549 & -0.134961146 & -0.117863445 \\
\hline 12.4 & 0 & 0.000529419 & -0.136062928 & -0.118955798 \\
\hline 12.5 & 0 & 0.000460939 & -0.137160932 & -0.120044374 \\
\hline 12.6 & 0 & 0.000393106 & -0.138255188 & -0.121129202 \\
\hline 12.7 & 0 & 0.000325915 & -0.139345722 & -0.12221031 \\
\hline 12.8 & 0 & 0.000259362 & -0.140432561 & -0.123287724 \\
\hline 12.9 & 0 & 0.000193442 & -0.141515734 & -0.124361472 \\
\hline 13 & 0 & 0.00012815 & -0.142595266 & -0.12543158 \\
\hline 13.1 & 0 & 0.0000634839 & -0.143671183 & -0.126498073 \\
\hline 13.2 & 0 & -0.0000005619 & -0.144743511 & -0.127560979 \\
\hline 13.3 & 0 & -0.0000639914 & -0.145812276 & -0.128620323 \\
\hline 13.4 & 0 & -0.000126808 & -0.146877504 & -0.12967613 \\
\hline 13.5 & 0 & -0.000189017 & -0.147939218 & -0.130728424 \\
\hline 13.6 & 0 & -0.000250621 & -0.148997444 & -0.131777231 \\
\hline 13.7 & 0 & -0.000311625 & -0.150052207 & -0.132822575 \\
\hline 13.8 & 0 & -0.000372033 & -0.151103529 & -0.13386448 \\
\hline 13.9 & 0 & -0.000431848 & -0.152151436 & -0.13490297 \\
\hline
\end{tabular}




\begin{tabular}{|c|c|c|c|c|}
\hline 14 & 0 & -0.000491074 & -0.15319595 & -0.135938069 \\
\hline 14.1 & 0 & -0.000549715 & -0.154237095 & -0.136969799 \\
\hline 14.2 & 0 & -0.000607775 & -0.155274894 & -0.137998184 \\
\hline 14.3 & 0 & -0.000665258 & -0.156309369 & -0.139023247 \\
\hline 14.4 & 0 & -0.000722166 & -0.157340543 & -0.140045009 \\
\hline 14.5 & 0 & -0.000778504 & -0.158368439 & -0.141063493 \\
\hline 14.6 & 0 & -0.000834276 & -0.159393078 & -0.142078722 \\
\hline 14.7 & 0 & -0.000889485 & -0.160414482 & -0.143090716 \\
\hline 14.8 & 0 & -0.000944134 & -0.161432672 & -0.144099498 \\
\hline 14.9 & 0 & -0.000998227 & -0.16244767 & -0.145105088 \\
\hline 15 & 0 & -0.001051767 & -0.163459496 & -0.146107509 \\
\hline 15.1 & 0 & -0.001104758 & -0.164468172 & -0.147106779 \\
\hline 15.2 & 0 & -0.001157203 & -0.165473718 & -0.14810292 \\
\hline 15.3 & 0 & -0.001209106 & -0.166476154 & -0.149095953 \\
\hline 15.4 & 0 & -0.001260469 & -0.167475501 & -0.150085897 \\
\hline 15.5 & 0 & -0.001311297 & -0.168471778 & -0.151072772 \\
\hline 15.6 & 0 & -0.001361591 & -0.169465005 & -0.152056598 \\
\hline 15.7 & 0 & -0.001411357 & -0.170455202 & -0.153037395 \\
\hline 15.8 & 0 & -0.001460595 & -0.171442387 & -0.154015182 \\
\hline 15.9 & 0 & -0.001509311 & -0.172426581 & -0.154989977 \\
\hline 16 & 0 & -0.001557507 & -0.173407801 & -0.1559618 \\
\hline 16.1 & 0 & -0.001605186 & -0.174386066 & -0.156930669 \\
\hline 16.2 & 0 & -0.00165235 & -0.175361395 & -0.157896603 \\
\hline 16.3 & 0 & -0.001699004 & -0.176333806 & -0.15885962 \\
\hline 16.4 & 0 & -0.00174515 & -0.177303318 & -0.159819739 \\
\hline 16.5 & 0 & -0.001790791 & -0.178269947 & -0.160776976 \\
\hline 16.6 & 0 & -0.00183593 & -0.179233712 & -0.161731351 \\
\hline 16.7 & 0 & -0.00188057 & -0.180194631 & -0.16268288 \\
\hline 16.8 & 0 & -0.001924713 & -0.181152721 & -0.16363158 \\
\hline 16.9 & 0 & -0.001968364 & -0.182107998 & -0.16457747 \\
\hline 17 & 0 & -0.002011523 & -0.183060481 & -0.165520566 \\
\hline 17.1 & 0 & -0.002054195 & -0.184010185 & -0.166460884 \\
\hline
\end{tabular}




$\begin{array}{lllll}17.2 & 0 & -0.002096382 & -0.184957128 & -0.167398442 \\ 17.3 & 0 & -0.002138086 & -0.185901326 & -0.168333256 \\ 17.4 & 0 & -0.002179311 & -0.186842795 & -0.169265343 \\ 17.5 & 0 & -0.002220059 & -0.187781552 & -0.170194718 \\ 17.6 & 0 & -0.002260334 & -0.188717612 & -0.171121398 \\ 17.7 & 0 & -0.002300136 & -0.189650992 & -0.172045399 \\ 17.8 & 0 & -0.00233947 & -0.190581708 & -0.172966736 \\ 17.9 & 0 & -0.002378337 & -0.191509774 & -0.173885425 \\ 18 & 0 & -0.00241674 & -0.192435206 & -0.174801481 \\ 18.1 & 0 & -0.002454682 & -0.19335802 & -0.17571492 \\ 18.2 & 0 & -0.002492166 & -0.194278231 & -0.176625757 \\ 18.3 & 0 & -0.002529193 & -0.195195853 & -0.177534006 \\ 18.4 & 0 & -0.002565767 & -0.196110902 & -0.178439684 \\ 18.5 & 0 & -0.00260189 & -0.197023393 & -0.179342803 \\ 18.6 & 0 & -0.002637563 & -0.197933339 & -0.18024338 \\ 18.7 & 0 & -0.002672791 & -0.198840755 & -0.181141428 \\ 18.8 & 0 & -0.002707574 & -0.199745656 & -0.182036962 \\ 18.9 & 0 & -0.002741915 & -0.200648056 & -0.182929995 \\ 19.9 & 0 & -0.002775818 & -0.201547969 & -0.183820543 \\ 19.1 & 0 & -0.002809283 & -0.202445408 & -0.184708619 \\ 19.2 & 0 & -0.002842314 & -0.203340389 & -0.185594236 \\ 19.3 & 0 & -0.002874912 & -0.204232923 & -0.186477409 \\ 19.4 & 0 & -0.002907081 & -0.205123025 & -0.187358151 \\ 19.5 & 0 & -0.002938821 & -0.206010709 & -0.188236475 \\ 19.6 & 0 & -0.002970136 & -0.206895987 & -0.189112395 \\ 19.9 & 0 & -0.003031498 & -0.208659379 & -0.190857074 \\ 19.003001027 & -0.207778872 & -0.189985923 \\ 19.003061549 & -0.209537519 & -0.19172586 \\ 19091183 & -0.210413306 & -0.192592293\end{array}$


The Gibbs free energy difference of the four polytypes of boron nitride ( $\mathrm{r}-\mathrm{BN}, \mathrm{h}-\mathrm{BN}, \mathrm{c}-\mathrm{BN}$, and w-BN) as a function of pressure ranging from 0 to $20 \mathrm{GPa}$, calculated with respect to $\mathrm{r}-\mathrm{BN}$ as a reference at a given temperature of 1200 Kelvin. The unit of the Gibbs free energy difference for the four polytypes of boron nitride is given in $\mathrm{eV} /$ atom.

\begin{tabular}{|c|c|c|c|c|}
\hline \multicolumn{2}{|c|}{ P(GPa) r-BN } & \multirow{2}{*}{$\begin{array}{l}\text { h-BN } \\
0.017555738\end{array}$} & \multirow{2}{*}{$\begin{array}{l}\text { c-BN } \\
0.050039456\end{array}$} & \multirow{2}{*}{$\begin{array}{l}\text { w-BN } \\
0.065835837\end{array}$} \\
\hline 0 & 0 & & & \\
\hline 0.1 & 0 & 0.01733791 & 0.047950228 & 0.063756032 \\
\hline 0.2 & 0 & 0.017121028 & 0.045880145 & 0.061695371 \\
\hline 0.3 & 0 & 0.016905213 & 0.043828657 & 0.059653306 \\
\hline 0.4 & 0 & 0.016690566 & 0.041795247 & 0.057629318 \\
\hline 0.5 & 0 & 0.016477173 & 0.039779425 & 0.055622919 \\
\hline 0.6 & 0 & 0.016265105 & 0.037780728 & 0.053633644 \\
\hline 0.7 & 0 & 0.016054419 & 0.035798714 & 0.051661054 \\
\hline 0.8 & 0 & 0.015845166 & 0.033832968 & 0.04970473 \\
\hline 0.9 & 0 & 0.015637386 & 0.03188309 & 0.047764276 \\
\hline 1 & 0 & 0.015431111 & 0.029948704 & 0.045839313 \\
\hline 1.1 & 0 & 0.015226369 & 0.028029446 & 0.043929479 \\
\hline 1.2 & 0 & 0.015023182 & 0.026124972 & 0.042034429 \\
\hline 1.3 & 0 & 0.014821565 & 0.024234953 & 0.040153833 \\
\hline 1.4 & 0 & 0.014621532 & 0.022359073 & 0.038287377 \\
\hline 1.5 & 0 & 0.014423092 & 0.020497029 & 0.036434757 \\
\hline 1.6 & 0 & 0.014226252 & 0.018648532 & 0.034595684 \\
\hline 1.7 & 0 & 0.014031015 & 0.016813303 & 0.032769879 \\
\hline 1.8 & 0 & 0.013837382 & 0.014991074 & 0.030957074 \\
\hline 1.9 & 0 & 0.013645353 & 0.01318159 & 0.029157013 \\
\hline 2 & 0 & 0.013454926 & 0.0113846 & 0.027369448 \\
\hline 2.1 & 0 & 0.013266097 & 0.009599869 & 0.025594141 \\
\hline 2.2 & 0 & 0.01307886 & 0.007827166 & 0.023830862 \\
\hline 2.3 & 0 & 0.01289321 & 0.006066269 & 0.022079389 \\
\hline 2.4 & 0 & 0.01270914 & 0.004316964 & 0.020339508 \\
\hline 2.5 & 0 & 0.012526641 & 0.002579046 & 0.018611014 \\
\hline 2.6 & 0 & 0.012345705 & 0.000852313 & 0.016893706 \\
\hline
\end{tabular}




\begin{tabular}{|c|c|c|c|c|}
\hline & 0 & 0.012166322 & -0.000863426 & 0.015187391 \\
\hline 2.8 & 0 & 0.011988483 & -0.002568358 & 0.013491883 \\
\hline 2.9 & 0 & 0.011812177 & -0.004262664 & 0.011807 \\
\hline 3 & 0 & 0.011637393 & -0.00594652 & 0.010132568 \\
\hline 3.1 & 0 & 0.01146412 & -0.007620095 & 0.008468417 \\
\hline 3.2 & 0 & 0.011292348 & -0.009283554 & 0.006814382 \\
\hline 3.3 & 0 & 0.011122063 & -0.010937056 & 0.005170303 \\
\hline 3.4 & 0 & 0.010953256 & -0.012580756 & 0.003536026 \\
\hline 3.5 & 0 & 0.010785913 & -0.014214806 & 0.0019114 \\
\hline 3.6 & 0 & 0.010620022 & -0.015839351 & 0.000296279 \\
\hline 3.7 & 0 & 0.010455573 & -0.017454532 & -0.001309479 \\
\hline 3.8 & 0 & 0.010292551 & -0.01906049 & -0.002906014 \\
\hline 3.9 & 0 & 0.010130946 & -0.020657357 & -0.004493458 \\
\hline 4 & 0 & 0.009970745 & -0.022245265 & -0.006071943 \\
\hline 4.1 & 0 & 0.009811936 & -0.023824341 & -0.007641596 \\
\hline 4.2 & 0 & 0.009654506 & -0.025394708 & -0.009202541 \\
\hline 4.3 & 0 & 0.009498444 & -0.026956489 & -0.0107549 \\
\hline 4.4 & 0 & 0.009343737 & -0.0285098 & -0.012298789 \\
\hline 4.5 & 0 & 0.009190373 & -0.030054757 & -0.013834323 \\
\hline 4.6 & 0 & 0.009038341 & -0.03159147 & -0.015361615 \\
\hline 4.7 & 0 & 0.008887628 & -0.03312005 & -0.016880773 \\
\hline 4.8 & 0 & 0.008738223 & -0.034640603 & -0.018391905 \\
\hline 4.9 & 0 & 0.008590113 & -0.036153233 & -0.019895114 \\
\hline 5 & 0 & 0.008443288 & -0.037658041 & -0.021390501 \\
\hline 5.1 & 0 & 0.008297736 & -0.039155127 & -0.022878166 \\
\hline 5.2 & 0 & 0.008153444 & -0.040644587 & -0.024358206 \\
\hline 5.3 & 0 & 0.008010403 & -0.042126516 & -0.025830715 \\
\hline 5.4 & 0 & 0.007868601 & -0.043601008 & -0.027295786 \\
\hline 5.5 & 0 & 0.007728026 & -0.045068151 & -0.02875351 \\
\hline 5.6 & 0 & 0.007588668 & -0.046528035 & -0.030203975 \\
\hline 5.7 & 0 & 0.007450515 & -0.047980747 & -0.031647267 \\
\hline 5.8 & 0 & 0.007313558 & -0.04942637 & -0.033083472 \\
\hline
\end{tabular}




\begin{tabular}{|c|c|c|c|c|}
\hline 5.9 & 0 & 0.007177785 & -0.050864989 & -0.034512671 \\
\hline 6 & 0 & 0.007043186 & -0.052296683 & -0.035934948 \\
\hline 6.1 & 0 & 0.006909751 & -0.053721533 & -0.03735038 \\
\hline 6.2 & 0 & 0.006777469 & -0.055139617 & -0.038759045 \\
\hline 6.3 & 0 & 0.00664633 & -0.05655101 & -0.040161021 \\
\hline 6.4 & 0 & 0.006516323 & -0.057955787 & -0.041556382 \\
\hline 6.5 & 0 & 0.006387441 & -0.059354022 & -0.0429452 \\
\hline 6.6 & 0 & 0.006259671 & -0.060745787 & -0.044327548 \\
\hline 6.7 & 0 & 0.006133006 & -0.062131151 & -0.045703496 \\
\hline 6.8 & 0 & 0.006007434 & -0.063510184 & -0.047073114 \\
\hline 6.9 & 0 & 0.005882947 & -0.064882953 & -0.048436468 \\
\hline 7 & 0 & 0.005759536 & -0.066249525 & -0.049793625 \\
\hline 7.1 & 0 & 0.005637191 & -0.067609965 & -0.05114465 \\
\hline 7.2 & 0 & 0.005515903 & -0.068964337 & -0.052489608 \\
\hline 7.3 & 0 & 0.005395663 & -0.070312704 & -0.053828561 \\
\hline 7.4 & 0 & 0.005276462 & -0.071655126 & -0.05516157 \\
\hline 7.5 & 0 & 0.005158293 & -0.072991666 & -0.056488696 \\
\hline 7.6 & 0 & 0.005041145 & -0.074322381 & -0.057809999 \\
\hline 7.7 & 0 & 0.00492501 & -0.075647331 & -0.059125537 \\
\hline 7.8 & 0 & 0.00480988 & -0.076966572 & -0.060435366 \\
\hline 7.9 & 0 & 0.004695747 & -0.078280162 & -0.061739544 \\
\hline 8 & 0 & 0.004582603 & -0.079588154 & -0.063038126 \\
\hline 8.1 & 0 & 0.004470439 & -0.080890605 & -0.064331166 \\
\hline 8.2 & 0 & 0.004359247 & -0.082187566 & -0.065618717 \\
\hline 8.3 & 0 & 0.004249019 & -0.083479091 & -0.066900833 \\
\hline 8.4 & 0 & 0.004139749 & -0.084765231 & -0.068177564 \\
\hline 8.5 & 0 & 0.004031427 & -0.086046037 & -0.069448961 \\
\hline 8.6 & 0 & 0.003924046 & -0.087321559 & -0.070715076 \\
\hline 8.7 & 0 & 0.003817599 & -0.088591847 & -0.071975955 \\
\hline 8.8 & 0 & 0.003712078 & -0.089856947 & -0.073231649 \\
\hline 8.9 & 0 & 0.003607477 & -0.091116909 & -0.074482204 \\
\hline 9 & 0 & 0.003503787 & -0.092371779 & -0.075727667 \\
\hline
\end{tabular}




\begin{tabular}{lllll}
9.1 & 0 & 0.003401002 & -0.093621602 & -0.076968085 \\
9.2 & 0 & 0.003299114 & -0.094866425 & -0.078203502 \\
9.3 & 0 & 0.003198117 & -0.096106291 & -0.079433964 \\
9.4 & 0 & 0.003098004 & -0.097341245 & -0.080659514 \\
9.5 & 0 & 0.002998768 & -0.09857133 & -0.081880195 \\
9.6 & 0 & 0.002900401 & -0.099796588 & -0.08309605 \\
9.7 & 0 & 0.002802899 & -0.101017062 & -0.084307122 \\
9.8 & 0 & 0.002706253 & -0.102232793 & -0.085513451 \\
9.9 & 0 & 0.002610458 & -0.103443821 & -0.086715078 \\
10 & 0 & 0.002515506 & -0.104650187 & -0.087912043 \\
10.1 & 0 & 0.002421393 & -0.10585193 & -0.089104385 \\
10.2 & 0 & 0.002328111 & -0.107049089 & -0.090292145 \\
10.3 & 0 & 0.002235654 & -0.108241702 & -0.091475359 \\
10.4 & 0 & 0.002144017 & -0.109429808 & -0.092654066 \\
10.5 & 0 & 0.002053193 & -0.110613443 & -0.093828303 \\
10.6 & 0 & 0.001963176 & -0.111792644 & -0.094998107 \\
10.7 & 0 & 0.001873961 & -0.112967447 & -0.096163513 \\
10.8 & 0 & 0.001785541 & -0.114137889 & -0.097324559 \\
10.9 & 0 & 0.00169791 & -0.115304004 & -0.098481278 \\
11 & 0 & 0.001611064 & -0.116465827 & -0.099633706 \\
11.1 & 0 & 0.001524997 & -0.117623392 & -0.100781877 \\
11.2 & 0 & 0.001439702 & -0.118776733 & -0.101925825 \\
11.3 & 0 & 0.001355175 & -0.119925884 & -0.103065582 \\
11.4 & 0 & 0.00127141 & -0.121070877 & -0.104201183 \\
11.5 & 0 & 0.001188402 & -0.122211745 & -0.105332659 \\
11.6 & 0 & 0.001106145 & -0.123348519 & -0.106460042 \\
11.7 & 0 & 0.001024634 & -0.124481231 & -0.107583364 \\
11.8 & 0 & 0.000943864 & -0.125609913 & -0.108702657 \\
\hline 1.9 & 0 & 0.000863831 & -0.126734596 & -0.10981795 \\
\hline 12 & 0 & 0.000784528 & -0.127855308 & -0.110929274 \\
\hline 10.1 & 0 & 0.00070595 & -0.128972082 & -0.112036659 \\
\hline 10.000628094 & -0.130084945 & -0.113140136
\end{tabular}




\begin{tabular}{|c|c|c|c|c|}
\hline 2.3 & 0 & 0.000550953 & -0.131193927 & -0.114239732 \\
\hline 12.4 & 0 & 0.000474524 & -0.132299058 & -0.115335477 \\
\hline 12.5 & 0 & 0.000398801 & -0.133400365 & -0.116427399 \\
\hline 12.6 & 0 & 0.000323779 & -0.134497877 & -0.117515527 \\
\hline 12.7 & 0 & 0.000249455 & -0.135591622 & -0.118599888 \\
\hline 12.8 & 0 & 0.000175823 & -0.136681626 & -0.11968051 \\
\hline 12.9 & 0 & 0.000102878 & -0.137767918 & -0.120757419 \\
\hline 13 & 0 & 0.0000306168 & -0.138850523 & -0.121830643 \\
\hline 13.1 & 0 & -0.0000409658 & -0.139929468 & -0.122900207 \\
\hline 13.2 & 0 & -0.000111874 & -0.141004779 & -0.123966139 \\
\hline 13.3 & 0 & -0.000182113 & -0.142076483 & -0.125028463 \\
\hline 13.4 & 0 & -0.000251686 & -0.143144604 & -0.126087206 \\
\hline 13.5 & 0 & -0.000320598 & -0.144209168 & -0.127142392 \\
\hline 13.6 & 0 & -0.000388852 & -0.1452702 & -0.128194047 \\
\hline 13.7 & 0 & -0.000456454 & -0.146327723 & -0.129242194 \\
\hline 13.8 & 0 & -0.000523407 & -0.147381763 & -0.130286859 \\
\hline 13.9 & 0 & -0.000589716 & -0.148432344 & -0.131328065 \\
\hline 14 & 0 & -0.000655385 & -0.149479489 & -0.132365837 \\
\hline 14.1 & 0 & -0.000720417 & -0.150523222 & -0.133400197 \\
\hline 14.2 & 0 & -0.000784816 & -0.151563566 & -0.134431168 \\
\hline 14.3 & 0 & -0.000848587 & -0.152600544 & -0.135458775 \\
\hline 14.4 & 0 & -0.000911734 & -0.153634179 & -0.136483039 \\
\hline 14.5 & 0 & -0.000974259 & -0.154664492 & -0.137503982 \\
\hline 14.6 & 0 & -0.001036168 & -0.155691507 & -0.138521628 \\
\hline 14.7 & 0 & -0.001097464 & -0.156715245 & -0.139535997 \\
\hline 14.8 & 0 & -0.00115815 & -0.157735727 & -0.140547113 \\
\hline 14.9 & 0 & -0.001218231 & -0.158752976 & -0.141554995 \\
\hline 15 & 0 & -0.00127771 & -0.159767013 & -0.142559666 \\
\hline 15.1 & 0 & -0.00133659 & -0.160777858 & -0.143561146 \\
\hline 15.2 & 0 & -0.001394876 & -0.161785532 & -0.144559456 \\
\hline 15.3 & 0 & -0.00145257 & -0.162790056 & -0.145554617 \\
\hline 15.4 & 0 & -0.001509678 & -0.16379145 & -0.146546648 \\
\hline
\end{tabular}




\begin{tabular}{|c|c|c|c|c|}
\hline 15.5 & 0 & -0.0015662 & -0.164789734 & -0.147535571 \\
\hline 15.6 & 0 & -0.001622143 & -0.165784928 & -0.148521404 \\
\hline 15.7 & 0 & -0.001677508 & -0.166777052 & -0.149504168 \\
\hline 15.8 & 0 & -0.001732299 & -0.167766126 & -0.150483883 \\
\hline 15.9 & 0 & -0.001786519 & -0.168752167 & -0.151460566 \\
\hline 16 & 0 & -0.001840173 & -0.169735196 & -0.152434238 \\
\hline 16.1 & 0 & -0.001893262 & -0.170715231 & -0.153404917 \\
\hline 16.2 & 0 & -0.001945791 & -0.171692292 & -0.154372621 \\
\hline 16.3 & 0 & -0.001997763 & -0.172666396 & -0.155337371 \\
\hline 16.4 & 0 & -0.00204918 & -0.173637561 & -0.156299182 \\
\hline 16.5 & 0 & -0.002100047 & -0.174605807 & -0.157258075 \\
\hline 16.6 & 0 & -0.002150366 & -0.17557115 & -0.158214066 \\
\hline 16.7 & 0 & -0.00220014 & -0.176533608 & -0.159167174 \\
\hline 16.8 & 0 & -0.002249372 & -0.1774932 & -0.160117415 \\
\hline 16.9 & 0 & -0.002298066 & -0.178449942 & -0.161064808 \\
\hline 17 & 0 & -0.002346224 & -0.179403852 & -0.16200937 \\
\hline 17.1 & 0 & -0.00239385 & -0.180354946 & -0.162951117 \\
\hline 17.2 & 0 & -0.002440946 & -0.181303242 & -0.163890066 \\
\hline 17.3 & 0 & -0.002487515 & -0.182248756 & -0.164826235 \\
\hline 17.4 & 0 & -0.002533561 & -0.183191505 & -0.165759639 \\
\hline 17.5 & 0 & -0.002579086 & -0.184131505 & -0.166690295 \\
\hline 17.6 & 0 & -0.002624093 & -0.185068773 & -0.16761822 \\
\hline 17.7 & 0 & -0.002668585 & -0.186003324 & -0.168543429 \\
\hline 17.8 & 0 & -0.002712564 & -0.186935174 & -0.169465938 \\
\hline 17.9 & 0 & -0.002756034 & -0.187864339 & -0.170385763 \\
\hline 18 & 0 & -0.002798997 & -0.188790834 & -0.17130292 \\
\hline 18.1 & 0 & -0.002841456 & -0.189714676 & -0.172217424 \\
\hline 18.2 & 0 & -0.002883414 & -0.19063588 & -0.17312929 \\
\hline 18.3 & 0 & -0.002924873 & -0.19155446 & -0.174038534 \\
\hline 18.4 & 0 & -0.002965837 & -0.192470431 & -0.174945171 \\
\hline 18.5 & 0 & -0.003006307 & -0.193383809 & -0.175849215 \\
\hline 18.6 & 0 & -0.003046286 & -0.194294609 & -0.176750681 \\
\hline
\end{tabular}




$\begin{array}{lllll}18.7 & 0 & -0.003085777 & -0.195202844 & -0.177649584 \\ 18.8 & 0 & -0.003124783 & -0.196108529 & -0.178545938 \\ 18.9 & 0 & -0.003163306 & -0.197011679 & -0.179439758 \\ 19 & 0 & -0.003201348 & -0.197912308 & -0.180331058 \\ 19.1 & 0 & -0.003238912 & -0.19881043 & -0.181219852 \\ 19.2 & 0 & -0.003276001 & -0.199706059 & -0.182106153 \\ 19.3 & 0 & -0.003312617 & -0.200599209 & -0.182989977 \\ 19.4 & 0 & -0.003348762 & -0.201489893 & -0.183871336 \\ 19.5 & 0 & -0.003384439 & -0.202378125 & -0.184750243 \\ 19.6 & 0 & -0.003419651 & -0.203263918 & -0.185626714 \\ 19.7 & 0 & -0.003454399 & -0.204147286 & -0.18650076 \\ 19.8 & 0 & -0.003488686 & -0.205028243 & -0.187372395 \\ 19.9 & 0 & -0.003522515 & -0.2059068 & -0.188241633 \\ 20 & 0 & -0.003555887 & -0.206782972 & -0.189108485\end{array}$

The Gibbs free energy difference of the four polytypes of boron nitride ( $\mathrm{r}-\mathrm{BN}, \mathrm{h}-\mathrm{BN}, \mathrm{c}-\mathrm{BN}$, and w-BN) as a function of pressure ranging from 0 to $20 \mathrm{GPa}$, calculated with respect to $\mathrm{r}-\mathrm{BN}$ as a reference at a given temperature of 1300 Kelvin. The unit of the Gibbs free energy difference for the four polytypes of boron nitride is given in $\mathrm{eV} /$ atom.

$\begin{array}{lllll}\mathbf{P}(\mathbf{G P a}) \mathbf{r}-\mathbf{B N} & \mathbf{h}-\mathbf{B N} & \mathbf{c}-\mathbf{B N} & \mathbf{w}-\mathbf{B N} \\ 0 & 0 & 0.018881183 & 0.054541242 & 0.070187607 \\ 0.1 & 0 & 0.018651729 & 0.052446412 & 0.068102182 \\ 0.2 & 0 & 0.018422945 & 0.050370422 & 0.066035595 \\ 0.3 & 0 & 0.018194983 & 0.048312753 & 0.063987331 \\ 0.4 & 0 & 0.017967971 & 0.046272915 & 0.061956898 \\ 0.5 & 0 & 0.01774202 & 0.044250444 & 0.059943832 \\ 0.6 & 0 & 0.017517221 & 0.042244899 & 0.057947692 \\ 0.7 & 0 & 0.017293655 & 0.04025586 & 0.055968059 \\ 0.8 & 0 & 0.017071388 & 0.038282929 & 0.054004534 \\ 0.9 & 0 & 0.016850478 & 0.036325726 & 0.052056737 \\ 1 & 0 & 0.016630973 & 0.034383886 & 0.050124304 \\ 1.1 & 0 & 0.016412912 & 0.032457063 & 0.048206887\end{array}$




\begin{tabular}{|c|c|c|c|c|}
\hline 1.2 & 0 & 0.016196331 & 0.030544925 & 0.046304156 \\
\hline 1.3 & 0 & 0.015981258 & 0.028647154 & 0.044415793 \\
\hline 1.4 & 0 & 0.015767715 & 0.026763445 & 0.042541492 \\
\hline 1.5 & 0 & 0.015555722 & 0.024893506 & 0.040680961 \\
\hline 1.6 & 0 & 0.015345295 & 0.023037056 & 0.038833919 \\
\hline 1.7 & 0 & 0.015136444 & 0.021193826 & 0.037000097 \\
\hline 1.8 & 0 & 0.014929179 & 0.019363556 & 0.035179235 \\
\hline 1.9 & 0 & 0.014723506 & 0.017545995 & 0.033371082 \\
\hline 2 & 0 & 0.014519429 & 0.015740903 & 0.031575399 \\
\hline 2.1 & 0 & 0.014316951 & 0.013948047 & 0.029791953 \\
\hline 2.2 & 0 & 0.014116071 & 0.012167205 & 0.028020519 \\
\hline 2.3 & 0 & 0.013916788 & 0.010398158 & 0.026260882 \\
\hline 2.4 & 0 & 0.013719101 & 0.0086407 & 0.024512833 \\
\hline 2.5 & 0 & 0.013523004 & 0.006894627 & 0.022776169 \\
\hline 2.6 & 0 & 0.013328494 & 0.005159743 & 0.021050695 \\
\hline 2.7 & 0 & 0.013135565 & 0.003435861 & 0.019336223 \\
\hline 2.8 & 0 & 0.01294421 & 0.001722797 & 0.017632568 \\
\hline 2.9 & 0 & 0.012754422 & 0.0000203725 & 0.015939554 \\
\hline 3 & 0 & 0.012566192 & -0.001671583 & 0.014257008 \\
\hline 3.1 & 0 & 0.012379514 & -0.003353237 & 0.012584763 \\
\hline 3.2 & 0 & 0.012194378 & -0.005024752 & 0.010922659 \\
\hline 3.3 & 0 & 0.012010774 & -0.006686285 & 0.009270537 \\
\hline 3.4 & 0 & 0.011828693 & -0.008337987 & 0.007628244 \\
\hline 3.5 & 0 & 0.011648125 & -0.009980008 & 0.005995633 \\
\hline 3.6 & 0 & 0.01146906 & -0.011612492 & 0.00437256 \\
\hline 3.7 & 0 & 0.011291488 & -0.013235579 & 0.002758883 \\
\hline 3.8 & 0 & 0.011115397 & -0.014849405 & 0.001154467 \\
\hline 3.9 & 0 & 0.010940777 & -0.016454104 & -0.000440822 \\
\hline 4 & 0 & 0.010767618 & -0.018049804 & -0.002027112 \\
\hline 4.1 & 0 & 0.010595908 & -0.019636631 & -0.003604529 \\
\hline 4.2 & 0 & 0.010425637 & -0.021214709 & -0.005173196 \\
\hline 4.3 & 0 & 0.010256792 & -0.022784156 & -0.006733234 \\
\hline
\end{tabular}




\begin{tabular}{|c|c|c|c|c|}
\hline & 0 & 0.010089364 & -0.02434509 & -0.008284758 \\
\hline 4.5 & 0 & 0.009923341 & -0.025897624 & -0.009827881 \\
\hline 4.6 & 0 & 0.009758711 & -0.027441868 & -0.011362716 \\
\hline 47 & & 0.009595464 & -0.028977932 & -0.01288937 \\
\hline 4.8 & 0 & 0.009433588 & -0.030505921 & -0.01440795 \\
\hline 4.9 & 0 & 0.009273073 & -0.032025938 & -0.015918557 \\
\hline 5 & 0 & 0.009113907 & -0.033538085 & -0.017421294 \\
\hline 5.1 & 0 & 0.00895608 & -0.035042458 & -0.018916258 \\
\hline 5.2 & 0 & 0.00879958 & -0.036539156 & -0.020403546 \\
\hline 5.3 & 0 & 0.008644396 & -0.038028271 & -0.021883253 \\
\hline 5.4 & 0 & 0.008490518 & -0.039509897 & -0.023355469 \\
\hline 5.5 & 0 & 0.008337935 & -0.040984123 & -0.024820286 \\
\hline 5.6 & 0 & 0.008186636 & -0.042451036 & -0.026277791 \\
\hline 5.7 & 0 & 0.00803661 & -0.043910724 & -0.027728071 \\
\hline 5.8 & 0 & 0.007887848 & -0.045363271 & -0.029171209 \\
\hline 5.9 & 0 & 0.007740339 & -0.046808759 & -0.030607289 \\
\hline 6 & 0 & 0.007594072 & -0.048247269 & -0.032036391 \\
\hline 6.1 & 0 & 0.007449037 & -0.049678881 & -0.033458595 \\
\hline 6.2 & 0 & 0.007305223 & -0.051103671 & -0.034873978 \\
\hline 6.3 & 0 & 0.007162622 & -0.052521717 & -0.036282616 \\
\hline 6.4 & 0 & 0.007021223 & -0.053933092 & -0.037684584 \\
\hline 6.5 & 0 & 0.006881016 & -0.05533787 & -0.039079955 \\
\hline 6.6 & 0 & 0.006741991 & -0.056736122 & -0.0404688 \\
\hline 6.7 & 0 & 0.006604139 & -0.058127918 & -0.04185119 \\
\hline 6.8 & 0 & 0.00646745 & -0.059513328 & -0.043227194 \\
\hline 6.9 & 0 & 0.006331915 & -0.06089242 & -0.04459688 \\
\hline 7 & 0 & 0.006197524 & -0.062265258 & -0.045960312 \\
\hline 7.1 & 0 & 0.006064269 & -0.063631909 & -0.047317558 \\
\hline 7.2 & 0 & 0.005932139 & -0.064992437 & -0.048668681 \\
\hline 7.3 & 0 & 0.005801126 & -0.066346904 & -0.050013742 \\
\hline 7.4 & 0 & 0.005671221 & -0.067695371 & -0.051352806 \\
\hline 7.5 & 0 & 0.005542415 & -0.0690379 & -0.05268593 \\
\hline
\end{tabular}




\begin{tabular}{|c|c|c|c|c|}
\hline 7.6 & 0 & 0.0054147 & -0.070374549 & -0.054013175 \\
\hline 7.7 & 0 & 0.005288066 & -0.071705378 & -0.0553346 \\
\hline 7.8 & 0 & 0.005162505 & -0.073030443 & -0.056650262 \\
\hline 7.9 & 0 & 0.005038009 & -0.0743498 & -0.057960216 \\
\hline 8 & 0 & 0.004914569 & -0.075663506 & -0.05926452 \\
\hline 8.1 & 0 & 0.004792177 & -0.076971614 & -0.060563226 \\
\hline 8.2 & 0 & 0.004670825 & -0.078274179 & -0.061856388 \\
\hline 8.3 & 0 & 0.004550505 & -0.079571252 & -0.06314406 \\
\hline 8.4 & 0 & 0.004431209 & -0.080862885 & -0.064426292 \\
\hline 8.5 & 0 & 0.004312928 & -0.08214913 & -0.065703137 \\
\hline 8.6 & 0 & 0.004195656 & -0.083430037 & -0.066974643 \\
\hline 8.7 & 0 & 0.004079384 & -0.084705654 & -0.06824086 \\
\hline 8.8 & 0 & 0.003964104 & -0.085976031 & -0.069501837 \\
\hline 8.9 & 0 & 0.00384981 & -0.087241214 & -0.070757621 \\
\hline 9 & 0 & 0.003736494 & -0.088501252 & -0.07200826 \\
\hline 9.1 & 0 & 0.003624148 & -0.089756189 & -0.073253799 \\
\hline 9.2 & 0 & 0.003512765 & -0.091006072 & -0.074494284 \\
\hline 9.3 & 0 & 0.003402338 & -0.092250945 & -0.075729759 \\
\hline 9.4 & 0 & 0.003292859 & -0.093490853 & -0.07696027 \\
\hline 9.5 & 0 & 0.003184323 & -0.094725838 & -0.078185859 \\
\hline 9.6 & 0 & 0.003076721 & -0.095955944 & -0.079406569 \\
\hline 9.7 & 0 & 0.002970047 & -0.097181213 & -0.080622442 \\
\hline 9.8 & 0 & 0.002864294 & -0.098401685 & -0.081833519 \\
\hline 9.9 & 0 & 0.002759455 & -0.099617403 & -0.083039842 \\
\hline 10 & 0 & 0.002655524 & -0.100828407 & -0.084241451 \\
\hline 10.1 & 0 & 0.002552495 & -0.102034735 & -0.085438386 \\
\hline 10.2 & 0 & 0.00245036 & -0.103236427 & -0.086630685 \\
\hline 10.3 & 0 & 0.002349113 & -0.104433522 & -0.087818387 \\
\hline 10.4 & 0 & 0.002248748 & -0.105626058 & -0.089001531 \\
\hline 10.5 & 0 & 0.002149259 & -0.106814072 & -0.090180153 \\
\hline 10.6 & 0 & 0.00205064 & -0.107997601 & -0.091354291 \\
\hline 10.7 & 0 & 0.001952883 & -0.109176682 & -0.09252398 \\
\hline
\end{tabular}




\begin{tabular}{|c|c|c|c|c|}
\hline 10.8 & 0 & 0.001855984 & -0.110351349 & -0.093689258 \\
\hline 10.9 & 0 & 0.001759936 & -0.11152164 & -0.094850159 \\
\hline 11 & 0 & 0.001664733 & -0.112687589 & -0.096006718 \\
\hline 11.1 & 0 & 0.00157037 & -0.113849229 & -0.09715897 \\
\hline 11.2 & 0 & 0.00147684 & -0.115006596 & -0.098306949 \\
\hline 1.3 & 0 & 0.001384138 & -0.116159722 & -0.099450688 \\
\hline 11.4 & 0 & 0.001292259 & -0.117308642 & -0.100590221 \\
\hline 11.5 & 0 & 0.001201196 & -0.118453386 & -0.101725579 \\
\hline 11.6 & 0 & 0.001110944 & -0.119593988 & -0.102856795 \\
\hline 11.7 & 0 & 0.001021497 & -0.12073048 & -0.103983902 \\
\hline 11.8 & 0 & 0.000932851 & -0.121862892 & -0.10510693 \\
\hline 11.9 & 0 & 0.000845 & -0.122991256 & -0.10622591 \\
\hline 12 & 0 & 0.000757937 & -0.124115603 & -0.107340873 \\
\hline 12.1 & 0 & 0.00067166 & -0.125235962 & -0.108451849 \\
\hline 12.2 & 0 & 0.00058616 & -0.126352363 & -0.109558868 \\
\hline 12.3 & 0 & 0.000501435 & -0.127464836 & -0.110661959 \\
\hline 12.4 & 0 & 0.000417479 & -0.128573409 & -0.111761152 \\
\hline 12.5 & 0 & 0.000334286 & -0.129678112 & -0.112856474 \\
\hline 12.6 & 0 & 0.000251852 & -0.130778972 & -0.113947955 \\
\hline 12.7 & 0 & 0.000170172 & -0.131876018 & -0.115035623 \\
\hline 12.8 & 0 & 0.0000892407 & -0.132969278 & -0.116119504 \\
\hline 12.9 & 0 & 0.0000090534 & -0.134058778 & -0.117199626 \\
\hline 13 & 0 & -0.0000703946 & -0.135144545 & -0.118276017 \\
\hline 13.1 & 0 & -0.000149108 & -0.136226607 & -0.119348702 \\
\hline 13.2 & 0 & -0.000227091 & -0.137304989 & -0.120417709 \\
\hline 13.3 & 0 & -0.00030435 & -0.138379718 & -0.121483063 \\
\hline 13.4 & 0 & -0.000380887 & -0.139450819 & -0.122544789 \\
\hline 13.5 & 0 & -0.000456709 & -0.140518317 & -0.123602914 \\
\hline 13.6 & 0 & -0.000531818 & -0.141582238 & -0.124657463 \\
\hline 13.7 & 0 & -0.000606221 & -0.142642607 & -0.125708459 \\
\hline 13.8 & 0 & -0.000679921 & -0.143699447 & -0.126755928 \\
\hline 13.9 & 0 & -0.000752922 & -0.144752784 & -0.127799894 \\
\hline
\end{tabular}




\begin{tabular}{|c|c|c|c|c|}
\hline 14 & 0 & -0.000825229 & -0.145802641 & -0.128840381 \\
\hline 14.1 & 0 & -0.000896846 & -0.146849042 & -0.129877413 \\
\hline 14.2 & 0 & -0.000967778 & -0.14789201 & -0.130911012 \\
\hline 14.3 & 0 & -0.001038028 & -0.148931568 & -0.131941203 \\
\hline 14.4 & 0 & -0.001107601 & -0.149967739 & -0.132968007 \\
\hline 14.5 & 0 & -0.001176501 & -0.151000547 & -0.133991448 \\
\hline 14.6 & 0 & -0.001244731 & -0.152030012 & -0.135011548 \\
\hline 14.7 & 0 & -0.001312296 & -0.153056159 & -0.13602833 \\
\hline 14.8 & 0 & -0.0013792 & -0.154079007 & -0.137041814 \\
\hline 14.9 & 0 & -0.001445447 & -0.155098579 & -0.138052023 \\
\hline 15 & 0 & -0.001511041 & -0.156114896 & -0.139058978 \\
\hline 15.1 & 0 & -0.001575985 & -0.15712798 & -0.1400627 \\
\hline 15.2 & 0 & -0.001640284 & -0.158137851 & -0.14106321 \\
\hline 15.3 & 0 & -0.00170394 & -0.159144531 & -0.14206053 \\
\hline 15.4 & 0 & -0.001766959 & -0.160148039 & -0.143054678 \\
\hline 15.5 & 0 & -0.001829343 & -0.161148396 & -0.144045677 \\
\hline 15.6 & 0 & -0.001891096 & -0.162145622 & -0.145033545 \\
\hline 15.7 & 0 & -0.001952223 & -0.163139737 & -0.146018303 \\
\hline 15.8 & 0 & -0.002012726 & -0.16413076 & -0.14699997 \\
\hline 15.9 & 0 & -0.002072609 & -0.165118711 & -0.147978566 \\
\hline 16 & 0 & -0.002131875 & -0.166103609 & -0.14895411 \\
\hline 16.1 & 0 & -0.002190529 & -0.167085474 & -0.149926621 \\
\hline 16.2 & 0 & -0.002248574 & -0.168064323 & -0.150896117 \\
\hline 16.3 & 0 & -0.002306013 & -0.169040176 & -0.151862619 \\
\hline 16.4 & 0 & -0.002362849 & -0.170013052 & -0.152826143 \\
\hline 16.5 & 0 & -0.002419087 & -0.170982967 & -0.153786708 \\
\hline 16.6 & 0 & -0.002474728 & -0.171949942 & -0.154744333 \\
\hline 16.7 & 0 & -0.002529778 & -0.172913992 & -0.155699035 \\
\hline 16.8 & 0 & -0.002584238 & -0.173875137 & -0.156650832 \\
\hline 16.9 & 0 & -0.002638112 & -0.174833394 & -0.157599742 \\
\hline 17 & 0 & -0.002691404 & -0.175788779 & -0.158545782 \\
\hline 17.1 & 0 & -0.002744116 & -0.176741311 & -0.159488969 \\
\hline
\end{tabular}




\begin{tabular}{|c|c|c|c|c|}
\hline 17.2 & 0 & -0.002796252 & -0.177691006 & -0.16042932 \\
\hline 17.3 & 0 & -0.002847815 & -0.178637882 & -0.161366852 \\
\hline 17.4 & 0 & -0.002898807 & -0.179581954 & -0.162301582 \\
\hline 17.5 & 0 & -0.002949234 & -0.180523239 & -0.163233526 \\
\hline 17.6 & 0 & -0.002999096 & -0.181461755 & -0.164162701 \\
\hline 17.7 & 0 & -0.003048397 & -0.182397516 & -0.165089122 \\
\hline 17.8 & 0 & -0.003097141 & -0.18333054 & -0.166012807 \\
\hline 17.9 & 0 & -0.00314533 & -0.184260842 & -0.166933771 \\
\hline 18 & 0 & -0.003192967 & -0.185188437 & -0.16785203 \\
\hline 18.1 & 0 & -0.003240055 & -0.186113342 & -0.168767599 \\
\hline 18.2 & 0 & -0.003286597 & -0.187035572 & -0.169680493 \\
\hline 18.3 & P & -0.003332596 & -0.187955142 & -0.170590729 \\
\hline 18.4 & P & -0.003378055 & -0.188872067 & -0.171498321 \\
\hline 18.5 & 0 & -0.003422976 & -0.189786363 & -0.172403285 \\
\hline 18.6 & 0 & -0.003467363 & -0.190698044 & -0.173305635 \\
\hline 18.7 & 0 & -0.003511217 & -0.191607126 & -0.174205386 \\
\hline 18.8 & 0 & -0.003554543 & -0.192513622 & -0.175102552 \\
\hline 18.9 & 0 & -0.003597342 & -0.193417547 & -0.175997149 \\
\hline 19 & 0 & -0.003639618 & -0.194318916 & -0.176889191 \\
\hline 19.1 & 0 & -0.003681373 & -0.195217743 & -0.177778691 \\
\hline 19.2 & 0 & -0.003722609 & -0.196114041 & -0.178665664 \\
\hline 19.3 & 0 & -0.00376333 & -0.197007826 & -0.179550124 \\
\hline 19.4 & 0 & -0.003803537 & -0.19789911 & -0.180432085 \\
\hline 19.5 & 0 & -0.003843235 & -0.198787908 & -0.18131156 \\
\hline 19.6 & 0 & -0.003882424 & -0.199674233 & -0.182188563 \\
\hline 19.7 & 0 & -0.003921108 & -0.200558099 & -0.183063108 \\
\hline 19.8 & 0 & -0.003959289 & -0.201439519 & -0.183935208 \\
\hline 19.9 & 0 & -0.00399697 & -0.202318505 & -0.184804876 \\
\hline & 0 & -0.004034154 & -0.203195073 & -0.185672126 \\
\hline
\end{tabular}


The Gibbs free energy difference of the four polytypes of boron nitride ( $\mathrm{r}-\mathrm{BN}, \mathrm{h}-\mathrm{BN}, \mathrm{c}-\mathrm{BN}$, and w-BN) as a function of pressure ranging from 0 to $20 \mathrm{GPa}$, calculated with respect to $\mathrm{r}-\mathrm{BN}$ as a reference at a given temperature of 1400 Kelvin. The unit of the Gibbs free energy difference for the four polytypes of boron nitride is given in $\mathrm{eV} / \mathrm{atom}$.

\begin{tabular}{lllll}
$\mathbf{P}(\mathbf{G P a}) \mathbf{r}-\mathbf{B N}$ & $\mathbf{h}-\mathbf{B N}$ & $\mathbf{c}-\mathbf{B N}$ & $\mathbf{w}-\mathbf{B N}$ \\
0 & 0 & 0.020225719 & 0.05897898 & 0.074484048 \\
0.1 & 0 & 0.019985318 & 0.056879377 & 0.072393833 \\
0.2 & 0 & 0.0197453 & 0.054798291 & 0.070322135 \\
0.3 & 0 & 0.019505845 & 0.052735236 & 0.068268468 \\
0.4 & 0 & 0.019267108 & 0.050689748 & 0.066232369 \\
0.5 & 0 & 0.019029222 & 0.048661387 & 0.064213398 \\
0.6 & 0 & 0.018792303 & 0.046649737 & 0.062211136 \\
0.7 & 0 & 0.018556448 & 0.044654396 & 0.060225185 \\
0.8 & 0 & 0.018321743 & 0.042674986 & 0.058255165 \\
0.9 & 0 & 0.018088262 & 0.040711142 & 0.056300712 \\
1 & 0 & 0.017856068 & 0.038762518 & 0.054361478 \\
1.1 & 0 & 0.017625215 & 0.03682878 & 0.052437132 \\
1.2 & 0 & 0.017395751 & 0.03490961 & 0.050527353 \\
1.3 & 0 & 0.017167714 & 0.033004703 & 0.048631837 \\
1.4 & 0 & 0.016941138 & 0.031113764 & 0.04675029 \\
1.5 & 0 & 0.016716054 & 0.029236512 & 0.04488243 \\
1.6 & 0 & 0.016492485 & 0.027372676 & 0.043027986 \\
1.7 & 0 & 0.016270451 & 0.025521993 & 0.041186696 \\
1.8 & 0 & 0.016049969 & 0.023684214 & 0.039358309 \\
1.9 & 0 & 0.015831052 & 0.021859096 & 0.037542584 \\
2.5 & 0 & 0.015613712 & 0.020046404 & 0.035739285 \\
2.1 & 0 & 0.015397957 & 0.018245914 & 0.033948188 \\
2.2 & 0 & 0.015183791 & 0.016457406 & 0.032169074 \\
2.3 & 0 & 0.014971221 & 0.014680672 & 0.030401733 \\
\hline .5 & 0 & 0.014760248 & 0.012915506 & 0.028645961 \\
\hline & 0 & 0.014550872 & 0.011161712 & 0.026901561 \\
0.014343094 & 0.009419099 & 0.025168342
\end{tabular}




\begin{tabular}{|c|c|c|c|c|}
\hline 2.7 & 0 & 0.014136912 & 0.007687482 & 0.023446119 \\
\hline 2.8 & 0 & 0.013932323 & 0.005966682 & 0.021734713 \\
\hline 2.9 & 0 & 0.013729323 & 0.004256525 & 0.020033951 \\
\hline 3 & 0 & 0.013527907 & 0.002556843 & 0.018343663 \\
\hline 3.1 & 0 & 0.01332807 & 0.000867471 & 0.016663686 \\
\hline 3.2 & 0 & 0.013129807 & -0.000811748 & 0.014993861 \\
\hline 3.3 & 0 & 0.012933109 & -0.00248097 & 0.013334034 \\
\hline 3.4 & 0 & 0.012737971 & -0.004140344 & 0.011684054 \\
\hline 3.5 & 0 & 0.012544384 & -0.005790017 & 0.010043776 \\
\hline 3.6 & 0 & 0.01235234 & -0.007430129 & 0.008413058 \\
\hline 3.7 & 0 & 0.012161831 & -0.00906082 & 0.006791762 \\
\hline 3.8 & 0 & 0.011972849 & -0.010682224 & 0.005179754 \\
\hline 3.9 & 0 & 0.011785383 & -0.012294471 & 0.003576901 \\
\hline 4 & 0 & 0.011599425 & -0.013897689 & 0.001983077 \\
\hline 4.1 & 0 & 0.011414965 & -0.015492003 & 0.000398158 \\
\hline 4.2 & 0 & 0.011231994 & -0.017077533 & -0.001177977 \\
\hline 4.3 & 0 & 0.011050501 & -0.018654399 & -0.002745447 \\
\hline 4.4 & 0 & 0.010870478 & -0.020222714 & -0.004304368 \\
\hline 4.5 & 0 & 0.010691913 & -0.021782591 & -0.005854851 \\
\hline 4.6 & 0 & 0.010514797 & -0.023334141 & -0.007397006 \\
\hline 4.7 & 0 & 0.010339119 & -0.02487747 & -0.00893094 \\
\hline 4.8 & 0 & 0.010164869 & -0.026412683 & -0.010456759 \\
\hline 4.9 & 0 & 0.009992038 & -0.027939883 & -0.011974564 \\
\hline 5 & 0 & 0.009820613 & -0.029459169 & -0.013484456 \\
\hline 5.1 & 0 & 0.009650586 & -0.030970639 & -0.014986532 \\
\hline 5.2 & 0 & 0.009481946 & -0.032474388 & -0.016480887 \\
\hline 5.3 & 0 & 0.009314683 & -0.033970511 & -0.017967616 \\
\hline 5.4 & 0 & 0.009148786 & -0.035459097 & -0.019446809 \\
\hline 5.5 & 0 & 0.008984245 & -0.036940238 & -0.020918555 \\
\hline 5.6 & 0 & 0.00882105 & -0.038414019 & -0.022382944 \\
\hline 5.7 & 0 & 0.00865919 & -0.039880528 & -0.023840059 \\
\hline 5.8 & 0 & 0.008498657 & -0.041339847 & -0.025289985 \\
\hline
\end{tabular}




\begin{tabular}{|c|c|c|c|c|}
\hline 5.9 & 0 & 0.008339438 & -0.04279206 & -0.026732804 \\
\hline 6 & 0 & 0.008181525 & -0.044237245 & -0.028168597 \\
\hline 6.1 & 0 & 0.008024908 & -0.045675483 & -0.029597442 \\
\hline 6.2 & 0 & 0.007869576 & -0.04710685 & -0.031019417 \\
\hline 6.3 & 0 & 0.00771552 & -0.048531423 & -0.032434597 \\
\hline 6.4 & 0 & 0.00756273 & -0.049949274 & -0.033843057 \\
\hline 6.5 & 0 & 0.007411197 & -0.051360478 & -0.035244869 \\
\hline 6.6 & 0 & 0.00726091 & -0.052765106 & -0.036640105 \\
\hline 6.7 & 0 & 0.00711186 & -0.054163228 & -0.038028835 \\
\hline 6.8 & 0 & 0.006964038 & -0.055554911 & -0.039411128 \\
\hline 6.9 & 0 & 0.006817435 & -0.056940225 & -0.040787051 \\
\hline 7 & 0 & 0.00667204 & -0.058319235 & -0.04215667 \\
\hline 7.1 & 0 & 0.006527846 & -0.059692005 & -0.04352005 \\
\hline 7.2 & 0 & 0.006384842 & -0.061058601 & -0.044877255 \\
\hline 7.3 & 0 & 0.00624302 & -0.062419084 & -0.046228348 \\
\hline 7.4 & 0 & 0.00610237 & -0.063773515 & -0.047573391 \\
\hline 7.5 & 0 & 0.005962885 & -0.065121956 & -0.048912442 \\
\hline 7.6 & 0 & 0.005824555 & -0.066464466 & -0.050245563 \\
\hline 7.7 & 0 & 0.005687371 & -0.067801103 & -0.051572811 \\
\hline 7.8 & 0 & 0.005551325 & -0.069131924 & -0.052894244 \\
\hline 7.9 & 0 & 0.005416408 & -0.070456986 & -0.054209918 \\
\hline 8 & 0 & 0.005282612 & -0.071776344 & -0.055519889 \\
\hline 8.1 & 0 & 0.005149929 & -0.073090052 & -0.05682421 \\
\hline 8.2 & 0 & 0.005018349 & -0.074398165 & -0.058122936 \\
\hline 8.3 & 0 & 0.004887866 & -0.075700734 & -0.059416119 \\
\hline 8.4 & 0 & 0.004758471 & -0.076997812 & -0.060703811 \\
\hline 8.5 & 0 & 0.004630156 & -0.078289449 & -0.061986062 \\
\hline 8.6 & 0 & 0.004502912 & -0.079575696 & -0.063262924 \\
\hline 8.7 & 0 & 0.004376733 & -0.080856602 & -0.064534445 \\
\hline 8.8 & 0 & 0.004251611 & -0.082132216 & -0.065800674 \\
\hline 8.9 & 0 & 0.004127536 & -0.083402585 & -0.067061658 \\
\hline 9 & 0 & 0.004004504 & -0.084667756 & -0.068317446 \\
\hline
\end{tabular}




\begin{tabular}{|c|c|c|c|c|}
\hline .1 & 0 & 0.003882504 & -0.085927776 & -0.069568082 \\
\hline 9.2 & 0 & 0.003761531 & -0.087182689 & -0.070813613 \\
\hline 9.3 & 0 & 0.003641576 & -0.088432542 & -0.072054083 \\
\hline 9.4 & 0 & 0.003522633 & -0.089677378 & -0.073289538 \\
\hline 9.5 & 0 & 0.003404695 & -0.090917241 & -0.074520019 \\
\hline 9.6 & 0 & 0.003287753 & -0.092152174 & -0.07574557 \\
\hline 9.7 & 0 & 0.003171801 & -0.093382218 & -0.076966234 \\
\hline 9.8 & 0 & 0.003056833 & -0.094607417 & -0.078182052 \\
\hline 9.9 & 0 & 0.002942841 & -0.095827809 & -0.079393065 \\
\hline 10 & 0 & 0.002829817 & -0.097043437 & -0.080599313 \\
\hline 10.1 & 0 & 0.002717757 & -0.098254339 & -0.081800837 \\
\hline 10.2 & 0 & 0.002606652 & -0.099460555 & -0.082997675 \\
\hline 10.3 & 0 & 0.002496497 & -0.100662124 & -0.084189866 \\
\hline 10.4 & 0 & 0.002387284 & -0.101859084 & -0.085377449 \\
\hline 10.5 & 0 & 0.002279008 & -0.103051472 & -0.08656046 \\
\hline 10.6 & 0 & 0.002171662 & -0.104239326 & -0.087738938 \\
\hline 10.7 & 0 & 0.002065238 & -0.105422682 & -0.088912919 \\
\hline 10.8 & 0 & 0.001959733 & -0.106601577 & -0.090082438 \\
\hline 10.9 & 0 & 0.001855138 & -0.107776045 & -0.091247531 \\
\hline 11 & 0 & 0.001751448 & -0.108946122 & -0.092408234 \\
\hline 11.1 & 0 & 0.001648656 & -0.110111842 & -0.093564581 \\
\hline 11.2 & 0 & 0.001546758 & -0.11127324 & -0.094716606 \\
\hline 11.3 & 0 & 0.001445746 & -0.11243035 & -0.095864343 \\
\hline 11.4 & 0 & 0.001345615 & -0.113583203 & -0.097007825 \\
\hline 11.5 & 0 & 0.001246359 & -0.114731835 & -0.098147086 \\
\hline 11.6 & 0 & 0.001147972 & -0.115876276 & -0.099282156 \\
\hline 11.7 & 0 & 0.001050449 & -0.117016559 & -0.100413069 \\
\hline 11.8 & 0 & 0.000953784 & -0.118152715 & -0.101539855 \\
\hline 11.9 & 0 & 0.000857971 & -0.119284775 & -0.102662547 \\
\hline 12 & 0 & 0.000763005 & -0.12041277 & -0.103781174 \\
\hline 12.1 & 0 & 0.00066888 & -0.121536731 & -0.104895768 \\
\hline 12.2 & 0 & 0.000575592 & -0.122656688 & -0.106006357 \\
\hline
\end{tabular}




\begin{tabular}{|c|c|c|c|c|}
\hline 12.3 & 0 & 0.000483133 & -0.123772669 & -0.107112972 \\
\hline 12.4 & 0 & 0.0003915 & -0.124884705 & -0.108215642 \\
\hline 12.5 & 0 & 0.000300687 & -0.125992824 & -0.109314396 \\
\hline 12.6 & 0 & 0.000210688 & -0.127097054 & -0.110409262 \\
\hline 12.7 & 0 & 0.000121499 & -0.128197424 & -0.111500268 \\
\hline 2.8 & 0 & 0.0000331149 & -0.129293962 & -0.112587443 \\
\hline 12.9 & 0 & -0.0000544702 & -0.130386695 & -0.113670813 \\
\hline 13 & 0 & -0.000141261 & -0.13147565 & -0.114750407 \\
\hline 13.1 & 0 & -0.000227262 & -0.132560854 & -0.11582625 \\
\hline 13.2 & 0 & -0.000312478 & -0.133642333 & -0.116898369 \\
\hline 13.3 & 0 & -0.000396915 & -0.134720114 & -0.11796679 \\
\hline 13.4 & 0 & -0.000480577 & -0.135794223 & -0.11903154 \\
\hline 13.5 & 0 & -0.000563469 & -0.136864684 & -0.120092643 \\
\hline 13.6 & 0 & -0.000645595 & -0.137931525 & -0.121150126 \\
\hline 13.7 & 0 & -0.00072696 & -0.138994768 & -0.122204013 \\
\hline 13.8 & 0 & -0.00080757 & -0.14005444 & -0.123254328 \\
\hline 13.9 & 0 & -0.000887428 & -0.141110564 & -0.124301097 \\
\hline 14 & 0 & -0.000966538 & -0.142163164 & -0.125344343 \\
\hline 14.1 & 0 & -0.001044906 & -0.143212265 & -0.12638409 \\
\hline 14.2 & 0 & -0.001122536 & -0.144257891 & -0.127420362 \\
\hline 14.3 & 0 & -0.001199432 & -0.145300063 & -0.128453182 \\
\hline 14.4 & 0 & -0.001275599 & -0.146338806 & -0.129482573 \\
\hline 14.5 & 0 & -0.001351041 & -0.147374142 & -0.130508558 \\
\hline 14.6 & 0 & -0.001425762 & -0.148406094 & -0.13153116 \\
\hline 14.7 & 0 & -0.001499766 & -0.149434683 & -0.1325504 \\
\hline 14.8 & 0 & -0.001573058 & -0.150459933 & -0.133566302 \\
\hline 14.9 & 0 & -0.001645641 & -0.151481865 & -0.134578886 \\
\hline 15 & 0 & -0.001717521 & -0.152500501 & -0.135588174 \\
\hline 15.1 & 0 & -0.0017887 & -0.153515861 & -0.136594188 \\
\hline 15.2 & 0 & -0.001859184 & -0.154527967 & -0.137596949 \\
\hline 15.3 & 0 & -0.001928975 & -0.155536841 & -0.138596478 \\
\hline 15.4 & 0 & -0.001998079 & -0.156542501 & -0.139592794 \\
\hline
\end{tabular}




\begin{tabular}{|c|c|c|c|c|}
\hline 15.5 & 0 & -0.002066498 & -0.15754497 & -0.14058592 \\
\hline 15.6 & 0 & -0.002134237 & -0.158544267 & -0.141575875 \\
\hline 15.7 & 0 & -0.0022013 & -0.159540412 & -0.142562679 \\
\hline 15.8 & 0 & -0.00226769 & -0.160533426 & -0.143546352 \\
\hline 15.9 & 0 & -0.002333411 & -0.161523327 & -0.144526913 \\
\hline 16 & 0 & -0.002398467 & -0.162510135 & -0.145504382 \\
\hline 16.1 & 0 & -0.002462862 & -0.163493869 & -0.146478779 \\
\hline 16.2 & 0 & -0.0025266 & -0.164474549 & -0.147450121 \\
\hline 16.3 & 0 & -0.002589683 & -0.165452193 & -0.148418429 \\
\hline 16.4 & 0 & -0.002652116 & -0.16642682 & -0.14938372 \\
\hline 16.5 & 0 & -0.002713902 & -0.167398448 & -0.150346013 \\
\hline 16.6 & 0 & -0.002775045 & -0.168367095 & -0.151305327 \\
\hline 16.7 & 0 & -0.002835548 & -0.16933278 & -0.152261679 \\
\hline 16.8 & 0 & -0.002895415 & -0.17029552 & -0.153215087 \\
\hline 16.9 & 0 & -0.002954649 & -0.171255334 & -0.15416557 \\
\hline 17 & 0 & -0.003013253 & -0.172212238 & -0.155113144 \\
\hline 17.1 & 0 & -0.003071232 & -0.17316625 & -0.156057827 \\
\hline 17.2 & 0 & -0.003128588 & -0.174117387 & -0.156999636 \\
\hline 17.3 & 0 & -0.003185325 & -0.175065667 & -0.157938588 \\
\hline 17.4 & 0 & -0.003241446 & -0.176011106 & -0.1588747 \\
\hline 17.5 & 0 & -0.003296954 & -0.176953721 & -0.159807989 \\
\hline 17.6 & 0 & -0.003351852 & -0.177893528 & -0.160738472 \\
\hline 17.7 & 0 & -0.003406145 & -0.178830544 & -0.161666164 \\
\hline 17.8 & 0 & -0.003459834 & -0.179764785 & -0.162591082 \\
\hline 17.9 & 0 & -0.003512924 & -0.180696268 & -0.163513242 \\
\hline 18 & 0 & -0.003565417 & -0.181625007 & -0.16443266 \\
\hline 18.1 & 0 & -0.003617316 & -0.182551019 & -0.165349352 \\
\hline 18.2 & 0 & -0.003668624 & -0.183474319 & -0.166263333 \\
\hline 18.3 & 0 & -0.003719346 & -0.184394923 & -0.167174619 \\
\hline 18.4 & 0 & -0.003769482 & -0.185312847 & -0.168083225 \\
\hline 18.5 & 0 & -0.003819037 & -0.186228104 & -0.168989167 \\
\hline 18.6 & 0 & -0.003868014 & -0.187140712 & -0.169892459 \\
\hline
\end{tabular}




$\begin{array}{lllll}18.7 & 0 & -0.003916416 & -0.188050684 & -0.170793116 \\ 18.8 & 0 & -0.003964244 & -0.188958034 & -0.171691154 \\ 18.9 & 0 & -0.004011504 & -0.189862779 & -0.172586586 \\ 19 & 0 & -0.004058196 & -0.190764932 & -0.173479428 \\ 19.1 & 0 & -0.004104324 & -0.191664508 & -0.174369693 \\ 19.2 & 0 & -0.004149892 & -0.192561521 & -0.175257396 \\ 19.3 & 0 & -0.004194901 & -0.193455984 & -0.176142551 \\ 19.4 & 0 & -0.004239355 & -0.194347913 & -0.177025173 \\ 19.5 & 0 & -0.004283255 & -0.195237321 & -0.177905274 \\ 19.6 & 0 & -0.004326607 & -0.196124222 & -0.178782869 \\ 19.7 & 0 & -0.00436941 & -0.197008629 & -0.179657972 \\ 19.8 & 0 & -0.004411669 & -0.197890555 & -0.180530595 \\ 19.9 & 0 & -0.004453387 & -0.198770015 & -0.181400753 \\ 20 & 0 & -0.004494565 & -0.199647022 & -0.182268458\end{array}$

The Gibbs free energy difference of the four polytypes of boron nitride ( $\mathrm{r}-\mathrm{BN}, \mathrm{h}-\mathrm{BN}, \mathrm{c}-\mathrm{BN}$, and w-BN) as a function of pressure ranging from 0 to $20 \mathrm{GPa}$, calculated with respect to $\mathrm{r}-\mathrm{BN}$ as a reference at a given temperature of 1500 Kelvin. The unit of the Gibbs free energy difference for the four polytypes of boron nitride is given in $\mathrm{eV} /$ atom.

$\begin{array}{lllll}\mathbf{P}(\mathbf{G P a}) \mathbf{r}-\mathbf{B N} & \mathbf{h}-\mathbf{B N} & \mathbf{c}-\mathbf{B N} & \mathbf{w}-\mathbf{B N} \\ 0 & 0 & 0.021583481 & 0.063358211 & 0.078720978 \\ 0.1 & 0 & 0.021332462 & 0.06125458 & 0.076626708 \\ 0.2 & 0 & 0.021081534 & 0.059169135 & 0.074550625 \\ 0.3 & 0 & 0.020830905 & 0.057101419 & 0.072492271 \\ 0.4 & 0 & 0.020580755 & 0.055050995 & 0.070451209 \\ 0.5 & 0 & 0.020331241 & 0.053017447 & 0.068427023 \\ 0.6 & 0 & 0.020082499 & 0.051000378 & 0.066419317 \\ 0.7 & 0 & 0.019834645 & 0.04899941 & 0.064427713 \\ 0.8 & 0 & 0.019587784 & 0.047014181 & 0.062451847 \\ 0.9 & 0 & 0.019342004 & 0.045044345 & 0.060491375 \\ 1 & 0 & 0.019097383 & 0.04308957 & 0.058545963 \\ 1.1 & 0 & 0.018853991 & 0.041149536 & 0.056615294\end{array}$




\begin{tabular}{|c|c|c|c|c|}
\hline 1.2 & 0 & 0.018611885 & 0.03922394 & 0.054699062 \\
\hline 1.3 & 0 & 0.018371117 & 0.037312487 & 0.052796974 \\
\hline 1.4 & 0 & 0.018131733 & 0.035414895 & 0.050908747 \\
\hline 1.5 & 0 & 0.017893771 & 0.033530893 & 0.04903411 \\
\hline 1.6 & 0 & 0.017657264 & 0.031660219 & 0.047172801 \\
\hline 1.7 & 0 & 0.01742224 & 0.029802621 & 0.045324569 \\
\hline 1.8 & 0 & 0.017188726 & 0.027957856 & 0.04348917 \\
\hline 1.9 & 0 & 0.016956741 & 0.02612569 & 0.04166637 \\
\hline 2 & 0 & 0.016726303 & 0.024305896 & 0.039855942 \\
\hline 2.1 & 0 & 0.016497427 & 0.022498255 & 0.038057667 \\
\hline 2.2 & 0 & 0.016270124 & 0.020702555 & 0.036271334 \\
\hline 2.3 & 0 & 0.016044405 & 0.018918592 & 0.034496738 \\
\hline 2.4 & 0 & 0.015820276 & 0.017146168 & 0.032733681 \\
\hline 2.5 & 0 & 0.015597744 & 0.01538509 & 0.03098197 \\
\hline 2.6 & 0 & 0.015376812 & 0.013635173 & 0.02924142 \\
\hline 2.7 & 0 & 0.015157483 & 0.011896236 & 0.02751185 \\
\hline 2.8 & 0 & 0.014939757 & 0.010168104 & 0.025793085 \\
\hline 2.9 & 0 & 0.014723635 & 0.008450607 & 0.024084956 \\
\hline 3 & 0 & 0.014509116 & 0.006743581 & 0.022387297 \\
\hline 3.1 & 0 & 0.014296196 & 0.005046865 & 0.020699948 \\
\hline 3.2 & 0 & 0.014084874 & 0.003360304 & 0.019022755 \\
\hline 3.3 & 0 & 0.013875144 & 0.001683746 & 0.017355564 \\
\hline 3.4 & 0 & 0.013667003 & 0.0000170436 & 0.01569823 \\
\hline 3.5 & 0 & 0.013460446 & -0.001639945 & 0.014050608 \\
\hline 3.6 & 0 & 0.013255465 & -0.003287361 & 0.01241256 \\
\hline 3.7 & 0 & 0.013052056 & -0.004925339 & 0.01078395 \\
\hline 3.8 & 0 & 0.012850211 & -0.006554011 & 0.009164646 \\
\hline 3.9 & 0 & 0.012649922 & -0.008173507 & 0.007554518 \\
\hline 4 & 0 & 0.012451184 & -0.009783951 & 0.005953441 \\
\hline 4.1 & 0 & 0.012253987 & -0.011385467 & 0.004361293 \\
\hline 4.2 & 0 & 0.012058323 & -0.012978173 & 0.002777954 \\
\hline 4.3 & 0 & 0.011864185 & -0.014562187 & 0.001203308 \\
\hline
\end{tabular}




\begin{tabular}{|c|c|c|c|c|}
\hline & 0 & 0.011671563 & -0.016137622 & -0.000362759 \\
\hline 4.5 & 0 & 0.011480449 & -0.017704588 & -0.001920358 \\
\hline 4.6 & 0 & 0.011290833 & -0.019263196 & -0.003469597 \\
\hline 47 & & 0.011102707 & -0.020813549 & -0.005010584 \\
\hline 4.8 & 0 & 0.010916061 & -0.022355753 & -0.00654342 \\
\hline 4.9 & 0 & 0.010730887 & -0.023889907 & -0.008068207 \\
\hline 5 & 0 & 0.010547173 & -0.025416111 & -0.009585044 \\
\hline 5.1 & 0 & 0.010364912 & -0.026934462 & -0.011094028 \\
\hline 5.2 & 0 & 0.010184093 & -0.028445055 & -0.012595253 \\
\hline 5.3 & 0 & 0.010004708 & -0.029947981 & -0.014088812 \\
\hline 5.4 & 0 & 0.009826746 & -0.031443331 & -0.015574796 \\
\hline 5.5 & 0 & 0.009650197 & -0.032931194 & -0.017053293 \\
\hline 5.6 & 0 & 0.009475052 & -0.034411658 & -0.01852439 \\
\hline 5.7 & 0 & 0.009301301 & -0.035884806 & -0.019988171 \\
\hline 5.8 & 0 & 0.009128936 & -0.037350722 & -0.021444721 \\
\hline 5.9 & 0 & 0.008957945 & -0.038809487 & -0.022894121 \\
\hline 6 & 0 & 0.00878832 & -0.040261183 & -0.02433645 \\
\hline 6.1 & 0 & 0.00862005 & -0.041705886 & -0.025771787 \\
\hline 6.2 & 0 & 0.008453127 & -0.043143673 & -0.02720021 \\
\hline 6.3 & 0 & 0.008287541 & -0.044574621 & -0.028621792 \\
\hline 6.4 & 0 & 0.008123282 & -0.045998802 & -0.030036608 \\
\hline 6.5 & 0 & 0.007960341 & -0.04741629 & -0.031444731 \\
\hline 6.6 & 0 & 0.007798708 & -0.048827155 & -0.032846231 \\
\hline 6.7 & 0 & 0.007638375 & -0.050231467 & -0.034241179 \\
\hline 6.8 & 0 & 0.007479332 & -0.051629294 & -0.035629642 \\
\hline 6.9 & 0 & 0.007321569 & -0.053020704 & -0.037011688 \\
\hline 7 & 0 & 0.007165078 & -0.054405762 & -0.038387382 \\
\hline 7.1 & 0 & 0.007009849 & -0.055784534 & -0.03975679 \\
\hline 7.2 & 0 & 0.006855874 & -0.057157082 & -0.041119976 \\
\hline 7.3 & 0 & 0.006703143 & -0.05852347 & -0.042477 \\
\hline 7.4 & 0 & 0.006551648 & -0.059883758 & -0.043827926 \\
\hline 7.5 & 0 & 0.00640138 & -0.061238008 & -0.045172813 \\
\hline
\end{tabular}




\begin{tabular}{|c|c|c|c|c|}
\hline 7.6 & 0 & 0.006252329 & -0.062586277 & -0.04651172 \\
\hline 7.7 & 0 & 0.006104489 & -0.063928625 & -0.047844706 \\
\hline 7.8 & 0 & 0.005957849 & -0.065265108 & -0.049171828 \\
\hline 7.9 & 0 & 0.005812401 & -0.066595783 & -0.050493141 \\
\hline 8 & 0 & 0.005668137 & -0.067920705 & -0.051808703 \\
\hline 8.1 & 0 & 0.005525048 & -0.069239929 & -0.053118566 \\
\hline 8.2 & 0 & 0.005383127 & -0.070553507 & -0.054422784 \\
\hline 8.3 & 0 & 0.005242365 & -0.071861494 & -0.055721411 \\
\hline 8.4 & 0 & 0.005102753 & -0.073163939 & -0.057014497 \\
\hline 8.5 & 0 & 0.004964284 & -0.074460896 & -0.058302094 \\
\hline 8.6 & 0 & 0.004826949 & -0.075752412 & -0.059584252 \\
\hline 8.7 & 0 & 0.004690742 & -0.077038539 & -0.060861019 \\
\hline 8.8 & 0 & 0.004555653 & -0.078319323 & -0.062132446 \\
\hline 8.9 & 0 & 0.004421675 & -0.079594814 & -0.063398579 \\
\hline 9 & 0 & 0.004288801 & -0.080865058 & -0.064659466 \\
\hline 9.1 & 0 & 0.004157022 & -0.082130102 & -0.065915152 \\
\hline 9.2 & 0 & 0.004026332 & -0.08338999 & -0.067165684 \\
\hline 9.3 & 0 & 0.003896722 & -0.084644769 & -0.068411107 \\
\hline 9.4 & 0 & 0.003768185 & -0.085894482 & -0.069651464 \\
\hline 9.5 & 0 & 0.003640715 & -0.087139173 & -0.0708868 \\
\hline 9.6 & 0 & 0.003514303 & -0.088378885 & -0.072117157 \\
\hline 9.7 & 0 & 0.003388942 & -0.08961366 & -0.073342578 \\
\hline 9.8 & 0 & 0.003264626 & -0.09084354 & -0.074563104 \\
\hline 9.9 & 0 & 0.003141347 & -0.092068566 & -0.075778776 \\
\hline 10 & 0 & 0.003019098 & -0.093288778 & -0.076989635 \\
\hline 10.1 & 0 & 0.002897873 & -0.094504217 & -0.078195721 \\
\hline 10.2 & 0 & 0.002777664 & -0.095714922 & -0.079397074 \\
\hline 10.3 & 0 & 0.002658465 & -0.096920931 & -0.080593731 \\
\hline 10.4 & 0 & 0.002540268 & -0.098122283 & -0.081785732 \\
\hline 10.5 & 0 & 0.002423068 & -0.099319015 & -0.082973113 \\
\hline 10.6 & 0 & 0.002306858 & -0.100511166 & -0.084155913 \\
\hline 10.7 & 0 & 0.002191631 & -0.101698771 & -0.085334168 \\
\hline
\end{tabular}




\begin{tabular}{|c|c|c|c|c|}
\hline 10.8 & 0 & 0.002077381 & -0.102881866 & -0.086507914 \\
\hline 10.9 & 0 & 0.001964101 & -0.104060487 & -0.087677187 \\
\hline 11 & 0 & 0.001851785 & -0.105234671 & -0.088842022 \\
\hline 11.1 & 0 & 0.001740427 & -0.10640445 & -0.090002453 \\
\hline 11.2 & 0 & 0.00163002 & -0.10756986 & -0.091158516 \\
\hline 11.3 & 0 & 0.001520558 & -0.108730934 & -0.092310243 \\
\hline 11.4 & 0 & 0.001412036 & -0.109887706 & -0.093457669 \\
\hline 11.5 & 0 & 0.001304448 & -0.111040209 & -0.094600826 \\
\hline 11.6 & 0 & 0.001197786 & -0.112188475 & -0.095739747 \\
\hline 11.7 & 0 & 0.001092046 & -0.113332536 & -0.096874464 \\
\hline 11.8 & 0 & 0.000987222 & -0.114472425 & -0.098005008 \\
\hline 11.9 & 0 & 0.000883307 & -0.115608171 & -0.099131411 \\
\hline 12 & 0 & 0.000780296 & -0.116739807 & -0.100253704 \\
\hline 12.1 & 0 & 0.000678183 & -0.117867362 & -0.101371917 \\
\hline 12.2 & 0 & 0.000576964 & -0.118990868 & -0.102486081 \\
\hline 12.3 & 0 & 0.000476631 & -0.120110352 & -0.103596224 \\
\hline 12.4 & 0 & 0.00037718 & -0.121225846 & -0.104702377 \\
\hline 12.5 & 0 & 0.000278605 & -0.122337378 & -0.105804569 \\
\hline 12.6 & 0 & 0.000180901 & -0.123444976 & -0.106902828 \\
\hline 12.7 & 0 & 0.0000840627 & -0.124548669 & -0.107997182 \\
\hline 12.8 & 0 & -0.000011916 & -0.125648485 & -0.10908766 \\
\hline 12.9 & 0 & -0.00010704 & -0.126744451 & -0.110174288 \\
\hline 13 & 0 & -0.000201314 & -0.127836595 & -0.111257096 \\
\hline 13.1 & 0 & -0.000294743 & -0.128924944 & -0.112336108 \\
\hline 13.2 & 0 & -0.000387334 & -0.130009524 & -0.113411353 \\
\hline 13.3 & 0 & -0.00047909 & -0.131090362 & -0.114482856 \\
\hline 13.4 & 0 & -0.000570016 & -0.132167484 & -0.115550643 \\
\hline 13.5 & 0 & -0.000660119 & -0.133240915 & -0.11661474 \\
\hline 13.6 & 0 & -0.000749402 & -0.134310681 & -0.117675174 \\
\hline 13.7 & 0 & -0.00083787 & -0.135376807 & -0.118731967 \\
\hline 13.8 & 0 & -0.000925529 & -0.136439318 & -0.119785147 \\
\hline 13.9 & 0 & -0.001012382 & -0.137498239 & -0.120834736 \\
\hline
\end{tabular}




\begin{tabular}{|c|c|c|c|c|}
\hline 4 & 0 & -0.001098436 & -0.138553593 & -0.12188076 \\
\hline 14.1 & 0 & -0.001183694 & -0.139605405 & -0.122923243 \\
\hline 14.2 & 0 & -0.001268161 & -0.140653699 & -0.123962207 \\
\hline 14.3 & 0 & -0.001351841 & -0.141698498 & -0.124997678 \\
\hline 14.4 & 0 & -0.00143474 & -0.142739825 & -0.126029677 \\
\hline 14.5 & 0 & -0.001516862 & -0.143777703 & -0.127058228 \\
\hline 14.6 & 0 & -0.001598211 & -0.144812155 & -0.128083354 \\
\hline 14.7 & 0 & -0.001678792 & -0.145843203 & -0.129105077 \\
\hline 14.8 & 0 & -0.001758608 & -0.14687087 & -0.130123419 \\
\hline 14.9 & 0 & -0.001837665 & -0.147895178 & -0.131138403 \\
\hline 15 & 0 & -0.001915967 & -0.148916148 & -0.132150049 \\
\hline 15.1 & 0 & -0.001993518 & -0.149933802 & -0.13315838 \\
\hline 15.2 & 0 & -0.002070322 & -0.15094816 & -0.134163417 \\
\hline 5.3 & 0 & -0.002146384 & -0.151959245 & -0.135165181 \\
\hline 15.4 & 0 & -0.002221707 & -0.152967077 & -0.136163693 \\
\hline 15.5 & 0 & -0.002296296 & -0.153971677 & -0.137158973 \\
\hline 15.6 & 0 & -0.002370155 & -0.154973064 & -0.138151041 \\
\hline 15.7 & 0 & -0.002443288 & -0.15597126 & -0.139139919 \\
\hline 15.8 & 0 & -0.002515698 & -0.156966284 & -0.140125625 \\
\hline 15.9 & 0 & -0.00258739 & -0.157958156 & -0.141108181 \\
\hline 16 & 0 & -0.002658368 & -0.158946895 & -0.142087604 \\
\hline 16.1 & 0 & -0.002728636 & -0.159932521 & -0.143063915 \\
\hline 16.2 & 0 & -0.002798197 & -0.160915053 & -0.144037133 \\
\hline 16.3 & 0 & -0.002867056 & -0.161894511 & -0.145007277 \\
\hline 16.4 & 0 & -0.002935216 & -0.162870911 & -0.145974365 \\
\hline 16.5 & 0 & -0.003002681 & -0.163844275 & -0.146938416 \\
\hline 16.6 & 0 & -0.003069455 & -0.164814619 & -0.147899449 \\
\hline 16.7 & 0 & -0.003135541 & -0.165781962 & -0.148857482 \\
\hline 16.8 & 0 & -0.003200943 & -0.166746322 & -0.149812533 \\
\hline 16.9 & 0 & -0.003265665 & -0.167707717 & -0.150764619 \\
\hline 17 & 0 & -0.00332971 & -0.168666165 & -0.151713759 \\
\hline 17.1 & 0 & -0.003393082 & -0.169621683 & -0.15265997 \\
\hline
\end{tabular}




\begin{tabular}{lllll}
17.2 & 0 & -0.003455784 & -0.170574288 & -0.153603269 \\
17.3 & 0 & -0.003517821 & -0.171523998 & -0.154543674 \\
17.4 & 0 & -0.003579195 & -0.17247083 & -0.155481201 \\
17.5 & 0 & -0.00363991 & -0.1734148 & -0.156415868 \\
17.6 & 0 & -0.003699969 & -0.174355926 & -0.157347691 \\
17.7 & 0 & -0.003759376 & -0.175294223 & -0.158276686 \\
17.8 & 0 & -0.003818135 & -0.176229708 & -0.15920287 \\
17.9 & 0 & -0.003876247 & -0.177162398 & -0.16012626 \\
18 & 0 & -0.003933718 & -0.178092308 & -0.161046871 \\
18.1 & 0 & -0.00399055 & -0.179019455 & -0.161964719 \\
18.2 & 0 & -0.004046745 & -0.179943854 & -0.16287982 \\
18.3 & 0 & -0.004102309 & -0.18086552 & -0.16379219 \\
18.4 & 0 & -0.004157243 & -0.18178447 & -0.164701843 \\
18.5 & 0 & -0.004211552 & -0.182700718 & -0.165608797 \\
18.6 & 0 & -0.004265237 & -0.18361428 & -0.166513065 \\
18.7 & 0 & -0.004318303 & -0.184525171 & -0.167414662 \\
18.8 & 0 & -0.004370752 & -0.185433406 & -0.168313605 \\
18.9 & 0 & -0.004422587 & -0.186338999 & -0.169209906 \\
19 & 0 & -0.004473812 & -0.187241965 & -0.170103582 \\
19.1 & 0 & -0.004524429 & -0.188142319 & -0.170994647 \\
19.2 & 0 & -0.004574443 & -0.189040075 & -0.171883114 \\
19.3 & 0 & -0.004623854 & -0.189935248 & -0.172768999 \\
19.4 & 0 & -0.004672667 & -0.190827851 & -0.173652315 \\
19.5 & 0 & -0.004720884 & -0.191717898 & -0.174533076 \\
19.6 & 0 & -0.004768509 & -0.192605404 & -0.175411298 \\
\hline 0.9 & 0 & -0.004815544 & -0.193490382 & -0.176286992 \\
\hline 10 & 0 & -0.004953138 & -0.196130285 & -0.178899048
\end{tabular}


The Gibbs free energy difference of the four polytypes of boron nitride ( $\mathrm{r}-\mathrm{BN}, \mathrm{h}-\mathrm{BN}, \mathrm{c}-\mathrm{BN}$, and w-BN) as a function of pressure ranging from 0 to $20 \mathrm{GPa}$, calculated with respect to $\mathrm{r}-\mathrm{BN}$ as a reference at a given temperature of $\mathbf{1 6 0 0}$ Kelvin. The unit of the Gibbs free energy difference for the four polytypes of boron nitride is given in $\mathrm{eV} /$ atom.

\begin{tabular}{|c|c|c|c|c|}
\hline \multicolumn{2}{|c|}{ P(GPa) r-BN } & \multirow{2}{*}{$\begin{array}{l}\text { h-BN } \\
0.022956347\end{array}$} & \multirow{2}{*}{$\begin{array}{l}\text { c-BN } \\
0.067687362\end{array}$} & \multirow{2}{*}{$\begin{array}{l}\text { w-BN } \\
0.082901936\end{array}$} \\
\hline 0 & 0 & & & \\
\hline 0.1 & 0 & 0.02269531 & 0.06558049 & 0.080804386 \\
\hline 0.2 & 0 & 0.022434056 & 0.063491454 & 0.078724671 \\
\hline 0.3 & 0 & 0.02217282 & 0.061419821 & 0.07666236 \\
\hline 0.4 & 0 & 0.021911805 & 0.059365182 & 0.074617044 \\
\hline 0.5 & 0 & 0.021651191 & 0.057327146 & 0.072588331 \\
\hline 0.6 & 0 & 0.021391135 & 0.055305337 & 0.070575846 \\
\hline 0.7 & 0 & 0.021131773 & 0.053299397 & 0.068579231 \\
\hline 0.8 & 0 & 0.020873225 & 0.051308984 & 0.066598143 \\
\hline 0.9 & 0 & 0.020615599 & 0.049333767 & 0.064632252 \\
\hline 1 & 0 & 0.020358985 & 0.047373431 & 0.062681242 \\
\hline 1.1 & 0 & 0.020103468 & 0.045427672 & 0.060744809 \\
\hline 1.2 & 0 & 0.019849118 & 0.043496199 & 0.058822663 \\
\hline 1.3 & 0 & 0.019595999 & 0.04157873 & 0.056914522 \\
\hline 1.4 & 0 & 0.019344167 & 0.039674996 & 0.055020115 \\
\hline 1.5 & 0 & 0.019093671 & 0.037784735 & 0.053139183 \\
\hline 1.6 & 0 & 0.018844553 & 0.035907696 & 0.051271473 \\
\hline 1.7 & 0 & 0.018596852 & 0.034043637 & 0.049416743 \\
\hline 1.8 & 0 & 0.018350601 & 0.032192324 & 0.047574759 \\
\hline 1.9 & 0 & 0.018105827 & 0.03035353 & 0.045745296 \\
\hline 2 & 0 & 0.017862556 & 0.028527037 & 0.043928133 \\
\hline 2.1 & 0 & 0.01762081 & 0.026712632 & 0.042123059 \\
\hline 2.2 & 0 & 0.017380605 & 0.024910111 & 0.04032987 \\
\hline 2.3 & 0 & 0.017141958 & 0.023119276 & 0.038548366 \\
\hline 2.4 & 0 & 0.016904882 & 0.021339935 & 0.036778356 \\
\hline 2.5 & 0 & 0.016669388 & 0.0195719 & 0.035019653 \\
\hline 2.6 & 0 & 0.016435483 & 0.017814991 & 0.033272077 \\
\hline
\end{tabular}




\begin{tabular}{|c|c|c|c|c|}
\hline 2.7 & 0 & 0.016203177 & 0.016069032 & 0.031535451 \\
\hline 2.8 & 0 & 0.015972472 & 0.014333854 & 0.029809606 \\
\hline 2.9 & 0 & 0.015743374 & 0.01260929 & 0.028094376 \\
\hline 3 & 0 & 0.015515885 & 0.01089518 & 0.026389599 \\
\hline 3.1 & 0 & 0.015290006 & 0.009191368 & 0.024695121 \\
\hline 3.2 & 0 & 0.015065737 & 0.007497701 & 0.023010788 \\
\hline 3.3 & 0 & 0.014843077 & 0.005814031 & 0.021336453 \\
\hline 3.4 & 0 & 0.014622025 & 0.004140215 & 0.019671972 \\
\hline 3.5 & 0 & 0.014402579 & 0.002476112 & 0.018017204 \\
\hline 3.6 & 0 & 0.014184734 & 0.000821586 & 0.016372014 \\
\hline 3.7 & 0 & 0.013968487 & -0.000823496 & 0.014736267 \\
\hline 3.8 & 0 & 0.013753834 & -0.002459264 & 0.013109834 \\
\hline 3.9 & 0 & 0.013540769 & -0.004085845 & 0.011492589 \\
\hline 4 & 0 & 0.013329287 & -0.005703362 & 0.009884408 \\
\hline 4.1 & 0 & 0.013119382 & -0.007311935 & 0.008285172 \\
\hline 4.2 & 0 & 0.012911047 & -0.008911681 & 0.006694762 \\
\hline 4.3 & 0 & 0.012704276 & -0.010502715 & 0.005113064 \\
\hline 4.4 & 0 & 0.012499062 & -0.01208515 & 0.003539966 \\
\hline 4.5 & 0 & 0.012295397 & -0.013659094 & 0.001975359 \\
\hline 4.6 & 0 & 0.012093274 & -0.015224655 & 0.000419135 \\
\hline 4.7 & 0 & 0.011892685 & -0.016781936 & -0.001128809 \\
\hline 4.8 & 0 & 0.011693622 & -0.01833104 & -0.002668576 \\
\hline 4.9 & 0 & 0.011496077 & -0.019872067 & -0.004200265 \\
\hline F & 0 & 0.011300041 & -0.021405114 & -0.005723975 \\
\hline 5.1 & 0 & 0.011105506 & -0.022930277 & -0.007239801 \\
\hline 5.2 & 0 & 0.010912463 & -0.02444765 & -0.008747836 \\
\hline 5.3 & 0 & 0.010720904 & -0.025957323 & -0.010248171 \\
\hline 5.4 & 0 & 0.01053082 & -0.027459386 & -0.011740897 \\
\hline 5.5 & 0 & 0.010342202 & -0.028953928 & -0.013226101 \\
\hline 5.6 & 0 & 0.01015504 & -0.030441034 & -0.014703869 \\
\hline 5.7 & 0 & 0.009969327 & -0.031920787 & -0.016174285 \\
\hline 5.8 & 0 & 0.009785053 & -0.033393272 & -0.017637432 \\
\hline
\end{tabular}




\begin{tabular}{|c|c|c|c|c|}
\hline 5.9 & 0 & 0.00960221 & -0.034858568 & -0.01909339 \\
\hline 6 & 0 & 0.009420787 & -0.036316754 & -0.020542239 \\
\hline 6.1 & 0 & 0.009240776 & -0.037767909 & -0.021984056 \\
\hline 6.2 & 0 & 0.009062168 & -0.039212108 & -0.023418917 \\
\hline 6.3 & 0 & 0.008884953 & -0.040649427 & -0.024846898 \\
\hline 6.4 & 0 & 0.008709124 & -0.042079938 & -0.026268072 \\
\hline 6.5 & 0 & 0.00853467 & -0.043503714 & -0.027682509 \\
\hline 6.6 & 0 & 0.008361583 & -0.044920824 & -0.029090282 \\
\hline 6.7 & 0 & 0.008189854 & -0.046331339 & -0.03049146 \\
\hline 6.8 & 0 & 0.008019473 & -0.047735326 & -0.031886109 \\
\hline 6.9 & 0 & 0.007850432 & -0.049132852 & -0.033274298 \\
\hline 7 & 0 & 0.007682722 & -0.050523983 & -0.034656091 \\
\hline 7.1 & 0 & 0.007516334 & -0.051908782 & -0.036031553 \\
\hline 7.2 & 0 & 0.007351259 & -0.053287314 & -0.037400748 \\
\hline 7.3 & 0 & 0.007187489 & -0.05465964 & -0.038763737 \\
\hline 7.4 & 0 & 0.007025015 & -0.056025822 & -0.040120582 \\
\hline 7.5 & 0 & 0.006863827 & -0.057385919 & -0.041471343 \\
\hline 7.6 & 0 & 0.006703918 & -0.058739991 & -0.042816077 \\
\hline 7.7 & 0 & 0.006545279 & -0.060088095 & -0.044154845 \\
\hline 7.8 & 0 & 0.006387902 & -0.061430289 & -0.045487702 \\
\hline 7.9 & 0 & 0.006231778 & -0.062766628 & -0.046814705 \\
\hline 8 & 0 & 0.006076898 & -0.064097168 & -0.048135909 \\
\hline 8.1 & 0 & 0.005923254 & -0.065421963 & -0.049451368 \\
\hline 8.2 & 0 & 0.005770838 & -0.066741066 & -0.050761136 \\
\hline 8.3 & 0 & 0.005619643 & -0.06805453 & -0.052065264 \\
\hline 8.4 & 0 & 0.005469659 & -0.069362406 & -0.053363805 \\
\hline 8.5 & 0 & 0.005320878 & -0.070664746 & -0.05465681 \\
\hline 8.6 & 0 & 0.005173294 & -0.071961599 & -0.055944328 \\
\hline 8.7 & 0 & 0.005026897 & -0.073253015 & -0.057226409 \\
\hline 8.8 & 0 & 0.004881679 & -0.074539041 & -0.058503102 \\
\hline 8.9 & 0 & 0.004737634 & -0.075819727 & -0.059774453 \\
\hline 9 & 0 & 0.004594753 & -0.077095118 & -0.06104051 \\
\hline
\end{tabular}




\begin{tabular}{|c|c|c|c|c|}
\hline .1 & 0 & 0.004453029 & -0.078365262 & -0.06230132 \\
\hline 9.2 & 0 & 0.004312453 & -0.079630203 & -0.063556928 \\
\hline 9.3 & 0 & 0.004173019 & -0.080889987 & -0.064807379 \\
\hline 9.4 & & 0.004034718 & -0.082144658 & -0.066052717 \\
\hline 9.5 & 0 & 0.003897545 & -0.083394259 & -0.067292986 \\
\hline 9.6 & 0 & 0.00376149 & -0.084638834 & -0.068528228 \\
\hline 9.7 & 0 & 0.003626547 & -0.085878425 & -0.069758486 \\
\hline 9.8 & 0 & 0.003492709 & -0.087113073 & -0.070983803 \\
\hline 9.9 & 0 & 0.003359968 & -0.088342819 & -0.072204218 \\
\hline 10 & 0 & 0.003228317 & -0.089567705 & -0.073419773 \\
\hline 10.1 & 0 & 0.00309775 & -0.09078777 & -0.074630507 \\
\hline 10.2 & 0 & 0.002968259 & -0.092003054 & -0.07583646 \\
\hline 10.3 & 0 & 0.002839837 & -0.093213594 & -0.077037671 \\
\hline 10.4 & 0 & 0.002712478 & -0.094419431 & -0.078234177 \\
\hline 10.5 & 0 & 0.002586175 & -0.095620601 & -0.079426018 \\
\hline 10.6 & 0 & 0.002460921 & -0.096817142 & -0.08061323 \\
\hline 10.7 & 0 & 0.002336709 & -0.09800909 & -0.081795849 \\
\hline 10.8 & 0 & 0.002213533 & -0.099196482 & -0.082973913 \\
\hline 10.9 & 0 & 0.002091386 & -0.100379354 & -0.084147456 \\
\hline 11 & 0 & 0.001970263 & -0.10155774 & -0.085316515 \\
\hline 11.1 & 0 & 0.001850155 & -0.102731676 & -0.086481124 \\
\hline 11.2 & 0 & 0.001731057 & -0.103901196 & -0.087641318 \\
\hline 11.3 & 0 & 0.001612963 & -0.105066334 & -0.088797129 \\
\hline 11.4 & 0 & 0.001495867 & -0.106227124 & -0.089948593 \\
\hline 11.5 & 0 & 0.001379762 & -0.107383598 & -0.091095741 \\
\hline 11.6 & 0 & 0.001264642 & -0.108535789 & -0.092238607 \\
\hline 11.7 & 0 & 0.001150501 & -0.109683729 & -0.093377223 \\
\hline 11.8 & 0 & 0.001037333 & -0.110827451 & -0.09451162 \\
\hline 11.9 & 0 & 0.000925132 & -0.111966985 & -0.09564183 \\
\hline 12 & 0 & 0.000813893 & -0.113102362 & -0.096767884 \\
\hline 12.1 & 0 & 0.000703609 & -0.114233614 & -0.097889813 \\
\hline 12.2 & 0 & 0.000594274 & -0.11536077 & -0.099007647 \\
\hline
\end{tabular}




\begin{tabular}{|c|c|c|c|c|}
\hline 2.3 & 0 & 0.000485883 & -0.11648386 & -0.100121415 \\
\hline 12.4 & 0 & 0.00037843 & -0.117602914 & -0.101231147 \\
\hline 12.5 & 0 & 0.00027191 & -0.118717961 & -0.102336873 \\
\hline 12.6 & 0 & 0.000166317 & -0.119829029 & -0.103438621 \\
\hline 12.7 & 0 & 0.0000616446 & -0.120936147 & -0.104536419 \\
\hline 12.8 & 0 & -0.0000421115 & -0.122039344 & -0.105630296 \\
\hline 12.9 & 0 & -0.000144957 & -0.123138646 & -0.10672028 \\
\hline 13 & 0 & -0.000246898 & -0.124234082 & -0.107806397 \\
\hline 13.1 & 0 & -0.000347939 & -0.125325678 & -0.108888675 \\
\hline 13.2 & 0 & -0.000448086 & -0.126413462 & -0.109967141 \\
\hline 13.3 & 0 & -0.000547343 & -0.127497459 & -0.111041821 \\
\hline 13.4 & 0 & -0.000645716 & -0.128577696 & -0.112112742 \\
\hline 13.5 & 0 & -0.000743211 & -0.129654198 & -0.113179929 \\
\hline 13.6 & 0 & -0.000839832 & -0.130726992 & -0.114243407 \\
\hline 13.7 & 0 & -0.000935584 & -0.131796103 & -0.115303203 \\
\hline 13.8 & 0 & -0.001030472 & -0.132861555 & -0.116359341 \\
\hline 13.9 & 0 & -0.001124502 & -0.133923373 & -0.117411846 \\
\hline 14 & 0 & -0.001217677 & -0.134981582 & -0.118460742 \\
\hline 14.1 & 0 & -0.001310004 & -0.136036206 & -0.119506053 \\
\hline 14.2 & 0 & -0.001401487 & -0.137087269 & -0.120547804 \\
\hline 14.3 & 0 & -0.00149213 & -0.138134794 & -0.121586018 \\
\hline 14.4 & 0 & -0.001581939 & -0.139178804 & -0.122620718 \\
\hline 14.5 & 0 & -0.001670918 & -0.140219324 & -0.123651927 \\
\hline 14.6 & 0 & -0.001759072 & -0.141256375 & -0.124679669 \\
\hline 14.7 & 0 & -0.001846405 & -0.14228998 & -0.125703965 \\
\hline 14.8 & 0 & -0.001932922 & -0.143320162 & -0.126724839 \\
\hline 14.9 & 0 & -0.002018627 & -0.144346943 & -0.127742312 \\
\hline 15 & 0 & -0.002103526 & -0.145370345 & -0.128756407 \\
\hline 15.1 & 0 & -0.002187622 & -0.146390389 & -0.129767145 \\
\hline 15.2 & 0 & -0.00227092 & -0.147407096 & -0.130774546 \\
\hline 15.3 & 0 & -0.002353424 & -0.148420488 & -0.131778634 \\
\hline 15.4 & 0 & -0.002435138 & -0.149430587 & -0.132779428 \\
\hline
\end{tabular}




\begin{tabular}{|c|c|c|c|c|}
\hline 15.5 & 0 & -0.002516068 & -0.150437412 & -0.133776949 \\
\hline 15.6 & 0 & -0.002596217 & -0.151440984 & -0.134771218 \\
\hline 15.7 & 0 & -0.002675589 & -0.152441324 & -0.135762256 \\
\hline 15.8 & 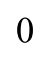 & -0.002754189 & -0.153438451 & -0.136750081 \\
\hline 15.9 & 0 & -0.00283202 & -0.154432386 & -0.137734715 \\
\hline 16 & 0 & -0.002909088 & -0.155423148 & -0.138716177 \\
\hline 16.1 & 0 & -0.002985395 & -0.156410757 & -0.139694486 \\
\hline 16.2 & 0 & -0.003060946 & -0.157395232 & -0.140669662 \\
\hline 16.3 & 0 & -0.003135746 & -0.158376592 & -0.141641724 \\
\hline 16.4 & 0 & -0.003209797 & -0.159354856 & -0.142610691 \\
\hline 16.5 & 0 & -0.003283104 & -0.160330044 & -0.143576581 \\
\hline 16.6 & 0 & -0.003355671 & -0.161302172 & -0.144539414 \\
\hline 16.7 & 0 & -0.003427502 & -0.162271261 & -0.145499207 \\
\hline 16.8 & 0 & -0.003498601 & -0.163237327 & -0.146455979 \\
\hline 16.9 & 0 & -0.003568971 & -0.164200389 & -0.147409747 \\
\hline 17 & 0 & -0.003638616 & -0.165160466 & -0.14836053 \\
\hline 17.1 & 0 & -0.00370754 & -0.166117573 & -0.149308345 \\
\hline 17.2 & 0 & -0.003775747 & -0.16707173 & -0.15025321 \\
\hline 17.3 & 0 & -0.00384324 & -0.168022953 & -0.151195142 \\
\hline 17.4 & 0 & -0.003910024 & -0.168971259 & -0.152134159 \\
\hline 17.5 & 0 & -0.003976101 & -0.169916666 & -0.153070276 \\
\hline 17.6 & 0 & -0.004041475 & -0.17085919 & -0.154003511 \\
\hline 17.7 & 0 & -0.00410615 & -0.171798848 & -0.154933882 \\
\hline 17.8 & 0 & -0.00417013 & -0.172735656 & -0.155861403 \\
\hline 17.9 & 0 & -0.004233417 & -0.173669632 & -0.156786092 \\
\hline 18 & 0 & -0.004296016 & -0.17460079 & -0.157707965 \\
\hline 18.1 & 0 & -0.00435793 & -0.175529148 & -0.158627038 \\
\hline 18.2 & 0 & -0.004419162 & -0.17645472 & -0.159543326 \\
\hline 18.3 & 0 & -0.004479715 & -0.177377523 & -0.160456846 \\
\hline 18.4 & 0 & -0.004539594 & -0.178297573 & -0.161367614 \\
\hline 18.5 & 0 & -0.004598801 & -0.179214884 & -0.162275644 \\
\hline 18.6 & 0 & -0.00465734 & -0.180129473 & -0.163180952 \\
\hline
\end{tabular}




$\begin{array}{lllll}18.7 & 0 & -0.004715214 & -0.181041354 & -0.164083553 \\ 18.8 & 0 & -0.004772426 & -0.181950542 & -0.164983462 \\ 18.9 & 0 & -0.00482898 & -0.182857053 & -0.165880695 \\ 19 & 0 & -0.004884879 & -0.183760901 & -0.166775265 \\ 19.1 & 0 & -0.004940125 & -0.184662101 & -0.167667188 \\ 19.2 & 0 & -0.004994723 & -0.185560667 & -0.168556479 \\ 19.3 & 0 & -0.005048675 & -0.186456614 & -0.169443151 \\ 19.4 & 0 & -0.005101984 & -0.187349955 & -0.170327218 \\ 19.5 & 0 & -0.005154654 & -0.188240706 & -0.171208696 \\ 19.6 & 0 & -0.005206687 & -0.18912888 & -0.172087598 \\ 19.7 & 0 & -0.005258087 & -0.190014491 & -0.172963938 \\ 19.8 & 0 & -0.005308857 & -0.190897553 & -0.173837729 \\ 19.9 & 0 & -0.005358999 & -0.191778079 & -0.174708986 \\ 20 & 0 & -0.005408517 & -0.192656084 & -0.175577721\end{array}$

The Gibbs free energy difference of the four polytypes of boron nitride ( $\mathrm{r}-\mathrm{BN}, \mathrm{h}-\mathrm{BN}, \mathrm{c}-\mathrm{BN}$, and w-BN) as a function of pressure ranging from 0 to $20 \mathrm{GPa}$, calculated with respect to $\mathrm{r}-\mathrm{BN}$ as a reference at a given temperature of 1700 Kelvin. The unit of the Gibbs free energy difference for the four polytypes of boron nitride is given in $\mathrm{eV} /$ atom.

$\begin{array}{lllll}\mathbf{P}(\mathbf{G P a}) \mathbf{r}-\mathbf{B N} & \mathbf{h}-\mathbf{B N} & \mathbf{c}-\mathbf{B N} & \mathbf{w}-\mathbf{B N} \\ 0 & 0 & 0.024341992 & 0.071960768 & 0.087027379 \\ 0.1 & 0 & 0.024071448 & 0.069851327 & 0.084927259 \\ 0.2 & 0 & 0.023800375 & 0.067759361 & 0.082844615 \\ 0.3 & 0 & 0.023529034 & 0.065684468 & 0.080779045 \\ 0.4 & 0 & 0.023257652 & 0.063626263 & 0.078730164 \\ 0.5 & 0 & 0.02298643 & 0.061584376 & 0.076697602 \\ 0.6 & 0 & 0.022715544 & 0.059558454 & 0.074681007 \\ 0.7 & 0 & 0.022445149 & 0.057548159 & 0.072680039 \\ 0.8 & 0 & 0.022175383 & 0.055553166 & 0.070694375 \\ 0.9 & 0 & 0.021906366 & 0.053573161 & 0.0687237 \\ 1 & 0 & 0.021638208 & 0.051607846 & 0.066767715 \\ 1.1 & 0 & 0.021371002 & 0.04965693 & 0.064826131\end{array}$




\begin{tabular}{|c|c|c|c|c|}
\hline 1.2 & 0 & 0.021104835 & 0.047720136 & 0.062898669 \\
\hline 1.3 & 0 & 0.02083978 & 0.045797195 & 0.060985062 \\
\hline 1.4 & 0 & 0.020575905 & 0.043887848 & 0.05908505 \\
\hline 1.5 & 0 & 0.020313269 & 0.041991847 & 0.057198384 \\
\hline 1.6 & 0 & 0.020051925 & 0.040108949 & 0.055324823 \\
\hline 1.7 & 0 & 0.019791918 & 0.038238923 & 0.053464134 \\
\hline 1.8 & 0 & 0.019533291 & 0.036381542 & 0.051616091 \\
\hline 1.9 & 0 & 0.019276078 & 0.034536589 & 0.049780477 \\
\hline 2 & 0 & 0.019020314 & 0.032703853 & 0.047957081 \\
\hline 2.1 & 0 & 0.018766025 & 0.030883129 & 0.046145698 \\
\hline 2.2 & 0 & 0.018513236 & 0.02907422 & 0.04434613 \\
\hline 2.3 & 0 & 0.018261969 & 0.027276934 & 0.042558187 \\
\hline 2.4 & 0 & 0.018012242 & 0.025491084 & 0.04078168 \\
\hline 2.5 & 0 & 0.017764073 & 0.023716491 & 0.03901643 \\
\hline 2.6 & 0 & 0.017517473 & 0.021952977 & 0.037262262 \\
\hline 2.7 & 0 & 0.017272455 & 0.020200374 & 0.035519004 \\
\hline 2.8 & 0 & 0.017029028 & 0.018458515 & 0.033786492 \\
\hline 2.9 & 0 & 0.016787201 & 0.01672724 & 0.032064563 \\
\hline 3 & 0 & 0.01654698 & 0.015006391 & 0.030353063 \\
\hline 3.1 & 0 & 0.01630837 & 0.013295818 & 0.028651837 \\
\hline 3.2 & 0 & 0.016071374 & 0.01159537 & 0.026960739 \\
\hline 3.3 & 0 & 0.015835995 & 0.009904905 & 0.025279624 \\
\hline 3.4 & 0 & 0.015602234 & 0.008224281 & 0.02360835 \\
\hline 3.5 & 0 & 0.015370092 & 0.006553362 & 0.021946782 \\
\hline 3.6 & 0 & 0.015139568 & 0.004892014 & 0.020294785 \\
\hline 3.7 & 0 & 0.014910661 & 0.003240106 & 0.01865223 \\
\hline 3.8 & 0 & 0.014683368 & 0.001597512 & 0.017018989 \\
\hline 3.9 & 0 & 0.014457687 & -0.0000358914 & 0.015394939 \\
\hline 4 & 0 & 0.014233615 & -0.001660226 & 0.013779958 \\
\hline 4.1 & 0 & 0.014011147 & -0.00327561 & 0.012173929 \\
\hline 4.2 & 0 & 0.013790279 & -0.004882158 & 0.010576736 \\
\hline 4.3 & 0 & 0.013571007 & -0.006479984 & 0.008988266 \\
\hline
\end{tabular}




\begin{tabular}{|c|c|c|c|c|}
\hline & 0 & 0.013353324 & -0.008069196 & 0.00740841 \\
\hline 4.5 & 0 & 0.013137226 & -0.009649902 & 0.005837061 \\
\hline 4.6 & 0 & 0.012922706 & -0.011222208 & 0.004274112 \\
\hline 47 & & 0.012709758 & -0.012786216 & 0.002719461 \\
\hline 4.8 & 0 & 0.012498374 & -0.014342026 & 0.001173009 \\
\hline 4.9 & 0 & 0.012288549 & -0.015889738 & -0.000365344 \\
\hline 5 & 0 & 0.012080276 & -0.017429447 & -0.001895695 \\
\hline 5.1 & 0 & 0.011873546 & -0.018961248 & -0.003418136 \\
\hline 5.2 & 0 & 0.011668352 & -0.020485233 & -0.004932761 \\
\hline 5.3 & 0 & 0.011464687 & -0.022001491 & -0.006439659 \\
\hline 5.4 & 0 & 0.011262543 & -0.023510112 & -0.007938919 \\
\hline 5.5 & 0 & 0.011061911 & -0.025011181 & -0.009430628 \\
\hline 5.6 & 0 & 0.010862784 & -0.026504785 & -0.01091487 \\
\hline 5.7 & 0 & 0.010665155 & -0.027991005 & -0.012391729 \\
\hline 5.8 & 0 & 0.010469013 & -0.029469925 & -0.013861287 \\
\hline 5.9 & 0 & 0.010274351 & -0.030941623 & -0.015323622 \\
\hline 6 & 0 & 0.010081162 & -0.032406178 & -0.016778815 \\
\hline 6.1 & 0 & 0.009889435 & -0.033863666 & -0.01822694 \\
\hline 6.2 & 0 & 0.009699163 & -0.035314164 & -0.019668075 \\
\hline 6.3 & 0 & 0.009510338 & -0.036757746 & -0.021102293 \\
\hline 6.4 & 0 & 0.00932295 & -0.038194483 & -0.022529667 \\
\hline 6.5 & 0 & 0.009136992 & -0.039624448 & -0.023950268 \\
\hline 6.6 & 0 & 0.008952454 & -0.04104771 & -0.025364165 \\
\hline 6.7 & 0 & 0.008769328 & -0.042464337 & -0.026771429 \\
\hline 6.8 & 0 & 0.008587606 & -0.043874398 & -0.028172125 \\
\hline 6.9 & 0 & 0.008407279 & -0.045277959 & -0.029566321 \\
\hline 7 & 0 & 0.008228338 & -0.046675084 & -0.030954082 \\
\hline 7.1 & 0 & 0.008050775 & -0.048065839 & -0.032335471 \\
\hline 7.2 & 0 & 0.007874582 & -0.049450284 & -0.033710552 \\
\hline 7.3 & 0 & 0.007699749 & -0.050828483 & -0.035079385 \\
\hline 7.4 & 0 & 0.007526269 & -0.052200496 & -0.036442033 \\
\hline 7.5 & 0 & 0.007354133 & -0.053566383 & -0.037798554 \\
\hline
\end{tabular}




\begin{tabular}{|c|c|c|c|c|}
\hline 7.6 & 0 & 0.007183332 & -0.054926202 & -0.039149008 \\
\hline 7.7 & 0 & 0.007013859 & -0.056280011 & -0.040493451 \\
\hline 7.8 & 0 & 0.006845705 & -0.057627867 & -0.041831941 \\
\hline 7.9 & 0 & 0.006678861 & -0.058969826 & -0.043164534 \\
\hline 8 & 0 & 0.00651332 & -0.060305942 & -0.044491284 \\
\hline 8.1 & 0 & 0.006349073 & -0.06163627 & -0.045812245 \\
\hline 8.2 & 0 & 0.006186112 & -0.062960862 & -0.047127471 \\
\hline 8.3 & 0 & 0.006024429 & -0.064279771 & -0.048437014 \\
\hline 8.4 & 0 & 0.005864016 & -0.065593049 & -0.049740926 \\
\hline 8.5 & 0 & 0.005704865 & -0.066900745 & -0.051039256 \\
\hline 8.6 & 0 & 0.005546968 & -0.068202911 & -0.052332056 \\
\hline 8.7 & 0 & 0.005390317 & -0.069499595 & -0.053619374 \\
\hline 8.8 & 0 & 0.005234905 & -0.070790845 & -0.054901258 \\
\hline 8.9 & 0 & 0.005080723 & -0.072076709 & -0.056177756 \\
\hline 9 & 0 & 0.004927763 & -0.073357235 & -0.057448915 \\
\hline 9.1 & 0 & 0.004776019 & -0.074632467 & -0.058714782 \\
\hline 9.2 & 0 & 0.004625482 & -0.075902452 & -0.059975401 \\
\hline 9.3 & 0 & 0.004476146 & -0.077167235 & -0.061230818 \\
\hline 9.4 & 0 & 0.004328001 & -0.078426859 & -0.062481077 \\
\hline 9.5 & 0 & 0.004181042 & -0.079681369 & -0.063726221 \\
\hline 9.6 & 0 & 0.00403526 & -0.080930807 & -0.064966293 \\
\hline 9.7 & 0 & 0.003890648 & -0.082175215 & -0.066201336 \\
\hline 9.8 & 0 & 0.0037472 & -0.083414636 & -0.067431391 \\
\hline 9.9 & 0 & 0.003604907 & -0.084649109 & -0.0686565 \\
\hline 10 & 0 & 0.003463763 & -0.085878677 & -0.069876703 \\
\hline 10.1 & 0 & 0.00332376 & -0.087103378 & -0.071092039 \\
\hline 10.2 & 0 & 0.003184892 & -0.088323252 & -0.072302549 \\
\hline 10.3 & 0 & 0.003047151 & -0.089538339 & -0.073508271 \\
\hline 10.4 & 0 & 0.002910531 & -0.090748675 & -0.074709243 \\
\hline 10.5 & 0 & 0.002775025 & -0.0919543 & -0.075905504 \\
\hline 10.6 & 0 & 0.002640625 & -0.09315525 & -0.07709709 \\
\hline 10.7 & 0 & 0.002507325 & -0.094351563 & -0.078284039 \\
\hline
\end{tabular}




\begin{tabular}{|c|c|c|c|c|}
\hline 10.8 & 0 & 0.002375119 & -0.095543274 & -0.079466387 \\
\hline 10.9 & 0 & 0.002244 & -0.096730419 & -0.080644169 \\
\hline 11 & 0 & 0.002113961 & -0.097913033 & -0.081817421 \\
\hline 11.1 & 0 & 0.001984996 & -0.099091152 & -0.082986177 \\
\hline 11.2 & 0 & 0.001857097 & -0.100264811 & -0.084150473 \\
\hline 11.3 & 0 & 0.00173026 & -0.101434042 & -0.085310342 \\
\hline 11.4 & 0 & 0.001604477 & -0.102598879 & -0.086465818 \\
\hline 11.5 & 0 & 0.001479742 & -0.103759356 & -0.087616934 \\
\hline 11.6 & 0 & 0.001356049 & -0.104915505 & -0.088763722 \\
\hline 11.7 & 0 & 0.001233391 & -0.106067359 & -0.089906215 \\
\hline 11.8 & 0 & 0.001111764 & -0.10721495 & -0.091044445 \\
\hline 11.9 & 0 & 0.000991159 & -0.108358308 & -0.092178443 \\
\hline 12 & 0 & 0.000871572 & -0.109497465 & -0.09330824 \\
\hline 12.1 & P & 0.000752996 & -0.110632451 & -0.094433867 \\
\hline 12.2 & 0 & 0.000635426 & -0.111763298 & -0.095555355 \\
\hline 12.3 & 0 & 0.000518855 & -0.112890034 & -0.096672733 \\
\hline 12.4 & 0 & 0.000403278 & -0.11401269 & -0.09778603 \\
\hline 12.5 & 0 & 0.000288689 & -0.115131295 & -0.098895277 \\
\hline 12.6 & 0 & 0.000175082 & -0.116245877 & -0.100000502 \\
\hline 12.7 & 0 & 0.0000624519 & -0.117356465 & -0.101101733 \\
\hline 12.8 & 0 & -0.0000492076 & -0.118463088 & -0.102198999 \\
\hline 12.9 & 0 & -0.000159902 & -0.119565772 & -0.103292328 \\
\hline 13 & 0 & -0.000269636 & -0.120664547 & -0.104381747 \\
\hline 13.1 & 0 & -0.000378417 & -0.121759438 & -0.105467283 \\
\hline 13.2 & 0 & -0.000486248 & -0.122850473 & -0.106548963 \\
\hline 13.3 & 0 & -0.000593136 & -0.123937679 & -0.107626814 \\
\hline 13.4 & 0 & -0.000699086 & -0.125021081 & -0.108700862 \\
\hline 13.5 & 0 & -0.000804103 & -0.126100706 & -0.109771134 \\
\hline 13.6 & 0 & -0.000908192 & -0.127176579 & -0.110837654 \\
\hline 13.7 & 0 & -0.001011359 & -0.128248725 & -0.111900449 \\
\hline 13.8 & 0 & -0.00111361 & -0.129317171 & -0.112959542 \\
\hline 13.9 & 0 & -0.001214948 & -0.13038194 & -0.11401496 \\
\hline
\end{tabular}




\begin{tabular}{|c|c|c|c|c|}
\hline 4 & 0 & -0.001315379 & -0.131443057 & -0.115066727 \\
\hline 14.1 & 0 & -0.001414908 & -0.132500547 & -0.116114866 \\
\hline 14.2 & 0 & -0.001513541 & -0.133554433 & -0.117159403 \\
\hline 14.3 & 0 & -0.001611281 & -0.13460474 & -0.11820036 \\
\hline 14.4 & 0 & -0.001708135 & -0.13565149 & -0.119237761 \\
\hline 14.5 & 0 & -0.001804106 & -0.136694706 & -0.12027163 \\
\hline 14.6 & 0 & -0.0018992 & -0.137734413 & -0.121301989 \\
\hline 14.7 & 0 & -0.001993421 & -0.138770633 & -0.122328862 \\
\hline 14.8 & 0 & -0.002086774 & -0.139803387 & -0.12335227 \\
\hline 14.9 & 0 & -0.002179265 & -0.140832699 & -0.124372236 \\
\hline 15 & 0 & -0.002270897 & -0.14185859 & -0.125388782 \\
\hline 15.1 & 0 & -0.002361675 & -0.142881082 & -0.12640193 \\
\hline 15.2 & 0 & -0.002451603 & -0.143900197 & -0.127411701 \\
\hline 15.3 & 0 & -0.002540688 & -0.144915956 & -0.128418117 \\
\hline 15.4 & 0 & -0.002628932 & -0.145928379 & -0.129421198 \\
\hline 15.5 & 0 & -0.00271634 & -0.146937489 & -0.130420966 \\
\hline 15.6 & 0 & -0.002802917 & -0.147943306 & -0.131417442 \\
\hline 15.7 & 0 & -0.002888667 & -0.148945849 & -0.132410645 \\
\hline 15.8 & 0 & -0.002973594 & -0.14994514 & -0.133400596 \\
\hline 15.9 & 0 & -0.003057703 & -0.150941198 & -0.134387315 \\
\hline 16 & 0 & -0.003140999 & -0.151934044 & -0.135370822 \\
\hline 16.1 & 0 & -0.003223484 & -0.152923696 & -0.136351136 \\
\hline 16.2 & 0 & -0.003305165 & -0.153910174 & -0.137328278 \\
\hline 16.3 & 0 & -0.003386044 & -0.154893499 & -0.138302266 \\
\hline 16.4 & 0 & -0.003466126 & -0.155873687 & -0.139273119 \\
\hline 16.5 & 0 & -0.003545414 & -0.156850759 & -0.140240856 \\
\hline 16.6 & 0 & -0.003623915 & -0.157824734 & -0.141205496 \\
\hline 16.7 & 0 & -0.00370163 & -0.158795628 & -0.142167057 \\
\hline 16.8 & 0 & -0.003778564 & -0.159763462 & -0.143125559 \\
\hline 16.9 & 0 & -0.003854721 & -0.160728253 & -0.144081018 \\
\hline & 0 & -0.003930106 & -0.161690019 & -0.145033453 \\
\hline .1 & 0 & -0.004004721 & -0.162648778 & -0.145982881 \\
\hline
\end{tabular}




\begin{tabular}{|c|c|c|c|c|}
\hline 17.2 & 0 & -0.004078572 & -0.163604547 & -0.146929321 \\
\hline 17.3 & 0 & -0.004151661 & -0.164557344 & -0.147872789 \\
\hline 17.4 & 0 & -0.004223992 & -0.165507186 & -0.148813303 \\
\hline 17.5 & 0 & -0.004295571 & -0.166454091 & -0.149750881 \\
\hline 17.6 & 0 & -0.004366399 & -0.167398075 & -0.150685538 \\
\hline 17.7 & 0 & -0.004436481 & -0.168339155 & -0.151617293 \\
\hline 17.8 & 0 & -0.00450582 & -0.169277348 & -0.152546161 \\
\hline 17.9 & 0 & -0.004574421 & -0.170212669 & -0.153472159 \\
\hline 18 & 0 & -0.004642287 & -0.171145137 & -0.154395303 \\
\hline 18.1 & 0 & -0.004709421 & -0.172074766 & -0.15531561 \\
\hline 18.2 & 0 & -0.004775828 & -0.173001573 & -0.156233096 \\
\hline 18.3 & 0 & -0.00484151 & -0.173925573 & -0.157147776 \\
\hline 18.4 & 0 & -0.004906471 & -0.174846783 & -0.158059666 \\
\hline 18.5 & 0 & -0.004970715 & -0.175765218 & -0.158968782 \\
\hline 18.6 & 0 & -0.005034245 & -0.176680893 & -0.159875139 \\
\hline 18.7 & 0 & -0.005097065 & -0.177593824 & -0.160778753 \\
\hline 18.8 & 0 & -0.005159178 & -0.178504026 & -0.161679639 \\
\hline 18.9 & 0 & -0.005220587 & -0.179411514 & -0.162577812 \\
\hline 19 & 0 & -0.005281296 & -0.180316303 & -0.163473287 \\
\hline 19.1 & 0 & -0.005341309 & -0.181218408 & -0.164366078 \\
\hline 19.2 & 0 & -0.005400627 & -0.182117843 & -0.1652562 \\
\hline 19.3 & 0 & -0.005459256 & -0.183014622 & -0.166143669 \\
\hline 19.4 & 0 & -0.005517198 & -0.183908761 & -0.167028497 \\
\hline 19.5 & 0 & -0.005574456 & -0.184800274 & -0.1679107 \\
\hline 19.6 & 0 & -0.005631033 & -0.185689174 & -0.168790291 \\
\hline 19.7 & 0 & -0.005686933 & -0.186575475 & -0.169667285 \\
\hline 19.8 & 0 & -0.00574216 & -0.187459192 & -0.170541696 \\
\hline 19.9 & 0 & -0.005796715 & -0.188340339 & -0.171413536 \\
\hline & 0 & -0.005850602 & -0.189218929 & -0.172282821 \\
\hline
\end{tabular}


The Gibbs free energy difference of the four polytypes of boron nitride ( $\mathrm{r}-\mathrm{BN}, \mathrm{h}-\mathrm{BN}, \mathrm{c}-\mathrm{BN}$, and w-BN) as a function of pressure ranging from 0 to $20 \mathrm{GPa}$, calculated with respect to $\mathrm{r}-\mathrm{BN}$ as a reference at a given temperature of $\mathbf{1 8 0 0}$ Kelvin. The unit of the Gibbs free energy difference for the four polytypes of boron nitride is given in $\mathrm{eV} / \mathrm{atom}$.

\begin{tabular}{|c|c|c|c|c|}
\hline \multicolumn{2}{|c|}{ P(GPa) r-BN } & \multirow{2}{*}{$\begin{array}{l}\text { h-BN } \\
0.025744004\end{array}$} & \multirow{2}{*}{$\begin{array}{l}\text { c-BN } \\
0.076175721\end{array}$} & \multirow{2}{*}{$\begin{array}{l}\text { w-BN } \\
0.091099904\end{array}$} \\
\hline 0 & 0 & & & \\
\hline 0.1 & 0 & 0.025464569 & 0.074064511 & 0.088997978 \\
\hline 0.2 & 0 & 0.025184279 & 0.071970401 & 0.086913153 \\
\hline 0.3 & 0 & 0.024903418 & 0.069893015 & 0.084845053 \\
\hline 0.4 & 0 & 0.024622237 & 0.067831993 & 0.082793317 \\
\hline 0.5 & 0 & 0.024340958 & 0.065786988 & 0.0807576 \\
\hline 0.6 & 0 & 0.024059775 & 0.063757669 & 0.078737568 \\
\hline 0.7 & 0 & 0.023778863 & 0.061743715 & 0.076732903 \\
\hline 0.8 & 0 & 0.023498375 & 0.05974482 & 0.074743298 \\
\hline 0.9 & 0 & 0.023218449 & 0.057760689 & 0.072768456 \\
\hline 1 & 0 & 0.022939208 & 0.055791036 & 0.070808095 \\
\hline 1.1 & 0 & 0.02266076 & 0.053835588 & 0.068861938 \\
\hline 1.2 & 0 & 0.022383202 & 0.05189408 & 0.066929722 \\
\hline 1.3 & 0 & 0.022106622 & 0.049966257 & 0.065011192 \\
\hline 1.4 & 0 & 0.021831098 & 0.048051871 & 0.063106099 \\
\hline 1.5 & 0 & 0.021556699 & 0.046150685 & 0.061214207 \\
\hline 1.6 & 0 & 0.021283488 & 0.044262467 & 0.059335284 \\
\hline 1.7 & 0 & 0.02101152 & 0.042386996 & 0.057469107 \\
\hline 1.8 & 0 & 0.020740846 & 0.040524054 & 0.055615461 \\
\hline 1.9 & 0 & 0.020471509 & 0.038673433 & 0.053774137 \\
\hline 2 & 0 & 0.020203548 & 0.036834929 & 0.05194493 \\
\hline 2.1 & 0 & 0.019937001 & 0.035008348 & 0.050127646 \\
\hline 2.2 & 0 & 0.019671897 & 0.033193496 & 0.048322092 \\
\hline 2.3 & 0 & 0.019408265 & 0.03139019 & 0.046528085 \\
\hline 2.4 & 0 & 0.019146129 & 0.029598251 & 0.044745444 \\
\hline 2.5 & 0 & 0.018885512 & 0.027817502 & 0.042973995 \\
\hline 2.6 & 0 & 0.018626431 & 0.026047775 & 0.041213568 \\
\hline
\end{tabular}




\begin{tabular}{|c|c|c|c|c|}
\hline & 0 & 0.018368904 & 0.024288904 & 0.039463997 \\
\hline 2.8 & 0 & 0.018112945 & 0.022540729 & 0.037725124 \\
\hline 2.9 & 0 & 0.017858566 & 0.020803095 & 0.035996791 \\
\hline 3 & 0 & 0.017605778 & 0.019075849 & 0.034278846 \\
\hline 3.1 & 0 & 0.017354591 & 0.017358842 & 0.032571142 \\
\hline 3.2 & 0 & 0.017105011 & 0.015651932 & 0.030873534 \\
\hline 3.3 & 0 & 0.016857044 & 0.013954977 & 0.029185882 \\
\hline 3.4 & 0 & 0.016610695 & 0.01226784 & 0.027508049 \\
\hline 3.5 & 0 & 0.016365968 & 0.010590389 & 0.025839901 \\
\hline 3.6 & 0 & 0.016122865 & 0.008922493 & 0.024181309 \\
\hline 3.7 & 0 & 0.015881388 & 0.007264024 & 0.022532145 \\
\hline 3.8 & 0 & 0.015641537 & 0.005614859 & 0.020892285 \\
\hline 3.9 & 0 & 0.015403312 & 0.003974877 & 0.019261608 \\
\hline 4 & 0 & 0.015166712 & 0.00234396 & 0.017639996 \\
\hline 4.1 & 0 & 0.014931736 & 0.000721991 & 0.016027334 \\
\hline 4.2 & 0 & 0.014698381 & -0.000891141 & 0.014423508 \\
\hline 4.3 & 0 & 0.014466644 & -0.002495547 & 0.012828408 \\
\hline 4.4 & 0 & 0.014236522 & -0.004091336 & 0.011241926 \\
\hline 4.5 & 0 & 0.014008012 & -0.005678611 & 0.009663958 \\
\hline 4.6 & 0 & 0.013781108 & -0.007257477 & 0.008094399 \\
\hline 4.7 & 0 & 0.013555807 & -0.008828034 & 0.00653315 \\
\hline 4.8 & 0 & 0.013332103 & -0.010390381 & 0.00498011 \\
\hline 4.9 & 0 & 0.01310999 & -0.011944615 & 0.003435184 \\
\hline 5 & 0 & 0.012889464 & -0.01349083 & 0.001898278 \\
\hline 5.1 & 0 & 0.012670518 & -0.015029119 & 0.000369297 \\
\hline 5.2 & 0 & 0.012453145 & -0.016559573 & -0.001151848 \\
\hline 5.3 & 0 & 0.01223734 & -0.01808228 & -0.002665246 \\
\hline 5.4 & 0 & 0.012023096 & -0.019597328 & -0.004170985 \\
\hline 5.5 & 0 & 0.011810406 & -0.021104801 & -0.005669149 \\
\hline 5.6 & 0 & 0.011599262 & -0.022604785 & -0.007159824 \\
\hline 5.7 & 0 & 0.011389658 & -0.02409736 & -0.008643089 \\
\hline 5.8 & 0 & 0.011181587 & -0.025582608 & -0.010119027 \\
\hline
\end{tabular}




\begin{tabular}{|c|c|c|c|c|}
\hline 5.9 & 0 & 0.01097504 & -0.027060606 & -0.011587715 \\
\hline 6 & 0 & 0.010770011 & -0.028531433 & -0.013049233 \\
\hline 6.1 & 0 & 0.010566491 & -0.029995165 & -0.014503654 \\
\hline 6.2 & 0 & 0.010364474 & -0.031451875 & -0.015951054 \\
\hline 6.3 & 0 & 0.01016395 & -0.032901638 & -0.017391507 \\
\hline 6.4 & 0 & 0.009964913 & -0.034344525 & -0.018825083 \\
\hline 6.5 & 0 & 0.009767355 & -0.035780606 & -0.020251854 \\
\hline 6.6 & 0 & 0.009571267 & -0.037209951 & -0.021671889 \\
\hline 6.7 & 0 & 0.009376641 & -0.038632628 & -0.023085255 \\
\hline 6.8 & 0 & 0.00918347 & -0.040048704 & -0.02449202 \\
\hline 6.9 & 0 & 0.008991745 & -0.041458244 & -0.025892249 \\
\hline 7 & 0 & 0.008801458 & -0.042861312 & -0.027286007 \\
\hline 7.1 & 0 & 0.008612602 & -0.044257973 & -0.028673358 \\
\hline 7.2 & 0 & 0.008425167 & -0.045648288 & -0.030054362 \\
\hline 7.3 & 0 & 0.008239147 & -0.04703232 & -0.031429083 \\
\hline 7.4 & 0 & 0.008054532 & -0.048410127 & -0.032797579 \\
\hline 7.5 & 0 & 0.007871316 & -0.049781769 & -0.034159911 \\
\hline 7.6 & 0 & 0.007689488 & -0.051147305 & -0.035516136 \\
\hline 7.7 & 0 & 0.007509043 & -0.052506792 & -0.036866312 \\
\hline 7.8 & 0 & 0.007329972 & -0.053860285 & -0.038210495 \\
\hline 7.9 & 0 & 0.007152266 & -0.055207842 & -0.039548741 \\
\hline 8 & 0 & 0.006975917 & -0.056549515 & -0.040881104 \\
\hline 8.1 & 0 & 0.006800918 & -0.057885359 & -0.042207637 \\
\hline 8.2 & 0 & 0.006627261 & -0.059215427 & -0.043528395 \\
\hline 8.3 & 0 & 0.006454938 & -0.060539771 & -0.044843428 \\
\hline 8.4 & 0 & 0.00628394 & -0.061858441 & -0.046152788 \\
\hline 8.5 & 0 & 0.006114261 & -0.063171489 & -0.047456526 \\
\hline 8.6 & 0 & 0.005945892 & -0.064478963 & -0.04875469 \\
\hline 8.7 & 0 & 0.005778826 & -0.065780913 & -0.050047331 \\
\hline 8.8 & 0 & 0.005613054 & -0.067077388 & -0.051334496 \\
\hline 8.9 & 0 & 0.00544857 & -0.068368434 & -0.052616232 \\
\hline 9 & 0 & 0.005285365 & -0.069654098 & -0.053892586 \\
\hline
\end{tabular}




\begin{tabular}{|c|c|c|c|c|}
\hline .1 & 0 & 0.005123432 & -0.070934426 & -0.055163605 \\
\hline 9.2 & 0 & 0.004962763 & -0.072209464 & -0.056429334 \\
\hline 9.3 & 0 & 0.004803352 & -0.073479255 & -0.057689816 \\
\hline 9.4 & & 0.004645189 & -0.074743845 & -0.058945098 \\
\hline 9.5 & 0 & 0.004488269 & -0.076003277 & -0.060195221 \\
\hline 9.6 & 0 & 0.004332584 & -0.077257593 & -0.061440229 \\
\hline 9.7 & 0 & 0.004178125 & -0.078506837 & -0.062680164 \\
\hline 9.8 & 0 & 0.004024887 & -0.079751048 & -0.063915067 \\
\hline 9.9 & 0 & 0.003872862 & -0.080990268 & -0.06514498 \\
\hline 10 & 0 & 0.003722043 & -0.082224539 & -0.066369942 \\
\hline 10.1 & 0 & 0.003572422 & -0.083453899 & -0.067589995 \\
\hline 10.2 & 0 & 0.003423993 & -0.084678388 & -0.068805177 \\
\hline 10.3 & 0 & 0.003276748 & -0.085898045 & -0.070015527 \\
\hline 10.4 & 0 & 0.003130681 & -0.087112907 & -0.071221083 \\
\hline 10.5 & 0 & 0.002985785 & -0.088323014 & -0.072421883 \\
\hline 10.6 & 0 & 0.002842052 & -0.089528402 & -0.073617965 \\
\hline 10.7 & 0 & 0.002699477 & -0.090729108 & -0.074809365 \\
\hline 10.8 & 0 & 0.002558052 & -0.091925167 & -0.075996119 \\
\hline 10.9 & 0 & 0.002417771 & -0.093116617 & -0.077178264 \\
\hline 11 & 0 & 0.002278626 & -0.094303492 & -0.078355834 \\
\hline 11.1 & 0 & 0.002140612 & -0.095485827 & -0.079528864 \\
\hline 11.2 & 0 & 0.002003722 & -0.096663657 & -0.08069739 \\
\hline 11.3 & 0 & 0.00186795 & -0.097837016 & -0.081861444 \\
\hline 11.4 & 0 & 0.001733288 & -0.099005936 & -0.083021061 \\
\hline 11.5 & 0 & 0.00159973 & -0.100170452 & -0.084176274 \\
\hline 11.6 & 0 & 0.001467271 & -0.101330596 & -0.085327114 \\
\hline 11.7 & 0 & 0.001335904 & -0.1024864 & -0.086473616 \\
\hline 11.8 & 0 & 0.001205622 & -0.103637896 & -0.08761581 \\
\hline 11.9 & 0 & 0.00107642 & -0.104785116 & -0.088753728 \\
\hline 12 & 0 & 0.000948291 & -0.105928091 & -0.089887401 \\
\hline 12.1 & 0 & 0.00082123 & -0.107066851 & -0.09101686 \\
\hline 12.2 & 0 & 0.000695229 & -0.108201428 & -0.092142136 \\
\hline
\end{tabular}




\begin{tabular}{|c|c|c|c|c|}
\hline 2.3 & 0 & 0.000570284 & -0.10933185 & -0.093263258 \\
\hline 12.4 & 0 & 0.000446388 & -0.110458148 & -0.094380256 \\
\hline 12.5 & 0 & 0.000323536 & -0.11158035 & -0.095493159 \\
\hline 12.6 & 0 & 0.00020172 & -0.112698487 & -0.096601996 \\
\hline 12.7 & 0 & 0.0000809369 & -0.113812586 & -0.097706796 \\
\hline 12.8 & 0 & -0.0000388207 & -0.114922676 & -0.098807587 \\
\hline 12.9 & 0 & -0.000157558 & -0.116028784 & -0.099904398 \\
\hline 13 & 0 & -0.000275281 & -0.117130938 & -0.100997255 \\
\hline 13.1 & 0 & -0.000391995 & -0.118229166 & -0.102086186 \\
\hline 13.2 & 0 & -0.000507705 & -0.119323494 & -0.103171217 \\
\hline 13.3 & 0 & -0.000622418 & -0.12041395 & -0.104252377 \\
\hline 13.4 & 0 & -0.000736138 & -0.121500559 & -0.10532969 \\
\hline 13.5 & 0 & -0.000848871 & -0.122583347 & -0.106403184 \\
\hline 13.6 & 0 & -0.000960623 & -0.123662341 & -0.107472883 \\
\hline 13.7 & 0 & -0.001071399 & -0.124737566 & -0.108538813 \\
\hline 13.8 & 0 & -0.001181203 & -0.125809046 & -0.109601 \\
\hline 13.9 & 0 & -0.001290042 & -0.126876808 & -0.110659468 \\
\hline 14 & 0 & -0.001397921 & -0.127940874 & -0.111714242 \\
\hline 14.1 & 0 & -0.001504844 & -0.129001271 & -0.112765347 \\
\hline 14.2 & 0 & -0.001610818 & -0.130058022 & -0.113812806 \\
\hline 14.3 & 0 & -0.001715846 & -0.131111151 & -0.114856644 \\
\hline 14.4 & 0 & -0.001819935 & -0.132160681 & -0.115896883 \\
\hline 14.5 & 0 & -0.001923088 & -0.133206636 & -0.116933548 \\
\hline 14.6 & 0 & -0.002025312 & -0.134249039 & -0.117966661 \\
\hline 14.7 & 0 & -0.00212661 & -0.135287912 & -0.118996246 \\
\hline 14.8 & 0 & -0.002226989 & -0.136323278 & -0.120022324 \\
\hline 14.9 & 0 & -0.002326452 & -0.137355161 & -0.121044918 \\
\hline 15 & 0 & -0.002425004 & -0.13838358 & -0.122064051 \\
\hline 15.1 & 0 & -0.002522651 & -0.13940856 & -0.123079744 \\
\hline 15.2 & 0 & -0.002619397 & -0.140430121 & -0.124092018 \\
\hline 15.3 & 0 & -0.002715247 & -0.141448284 & -0.125100896 \\
\hline 15.4 & 0 & -0.002810205 & -0.142463071 & -0.126106398 \\
\hline
\end{tabular}




\begin{tabular}{|c|c|c|c|c|}
\hline 15.5 & 0 & -0.002904276 & -0.143474504 & -0.127108546 \\
\hline 15.6 & 0 & -0.002997464 & -0.144482601 & -0.128107361 \\
\hline 15.7 & 0 & -0.003089775 & -0.145487385 & -0.129102862 \\
\hline 15.8 & 0 & -0.003181212 & -0.146488876 & -0.13009507 \\
\hline 15.9 & 0 & -0.003271781 & -0.147487094 & -0.131084005 \\
\hline 16 & 0 & -0.003361485 & -0.148482058 & -0.132069688 \\
\hline 16.1 & 0 & -0.003450329 & -0.149473788 & -0.133052138 \\
\hline 16.2 & 0 & -0.003538318 & -0.150462305 & -0.134031375 \\
\hline 16.3 & 0 & -0.003625455 & -0.151447627 & -0.135007417 \\
\hline 16.4 & 0 & -0.003711745 & -0.152429773 & -0.135980285 \\
\hline 16.5 & 0 & -0.003797192 & -0.153408763 & -0.136949997 \\
\hline 16.6 & 0 & -0.003881801 & -0.154384615 & -0.137916572 \\
\hline 16.7 & 0 & -0.003965575 & -0.155357349 & -0.138880029 \\
\hline 16.8 & 0 & -0.00404852 & -0.156326981 & -0.139840385 \\
\hline 16.9 & 0 & -0.004130638 & -0.157293531 & -0.14079766 \\
\hline 17 & 0 & -0.004211934 & -0.158257017 & -0.141751871 \\
\hline 17.1 & 0 & -0.004292413 & -0.159217457 & -0.142703037 \\
\hline 17.2 & 0 & -0.004372077 & -0.160174868 & -0.143651175 \\
\hline 17.3 & 0 & -0.004450932 & -0.161129267 & -0.144596302 \\
\hline 17.4 & 0 & -0.004528982 & -0.162080673 & -0.145538436 \\
\hline 17.5 & 0 & -0.004606229 & -0.163029103 & -0.146477594 \\
\hline 17.6 & 0 & -0.004682678 & -0.163974573 & -0.147413794 \\
\hline 17.7 & 0 & -0.004758333 & -0.164917101 & -0.148347052 \\
\hline 17.8 & 0 & -0.004833198 & -0.165856703 & -0.149277385 \\
\hline 17.9 & 0 & -0.004907277 & -0.166793396 & -0.15020481 \\
\hline 18 & 0 & -0.004980573 & -0.167727196 & -0.151129343 \\
\hline 18.1 & 0 & -0.00505309 & -0.16865812 & -0.152051 \\
\hline 18.2 & 0 & -0.005124832 & -0.169586184 & -0.152969798 \\
\hline 18.3 & 0 & -0.005195803 & -0.170511403 & -0.153885752 \\
\hline 18.4 & 0 & -0.005266006 & -0.171433795 & -0.154798879 \\
\hline 18.5 & 0 & -0.005335445 & -0.172353373 & -0.155709194 \\
\hline 18.6 & 0 & -0.005404124 & -0.173270155 & -0.156616713 \\
\hline
\end{tabular}




$\begin{array}{lllll}18.7 & 0 & -0.005472046 & -0.174184155 & -0.157521451 \\ 18.8 & 0 & -0.005539215 & -0.175095389 & -0.158423423 \\ 18.9 & 0 & -0.005605634 & -0.176003871 & -0.159322645 \\ 19 & 0 & -0.005671306 & -0.176909618 & -0.160219132 \\ 19.1 & 0 & -0.005736237 & -0.177812643 & -0.161112898 \\ 19.2 & 0 & -0.005800427 & -0.178712962 & -0.162003959 \\ 19.3 & 0 & -0.005863883 & -0.179610589 & -0.162892329 \\ 19.4 & 0 & -0.005926606 & -0.180505539 & -0.163778022 \\ 19.5 & 0 & -0.0059886 & -0.181397826 & -0.164661053 \\ 19.6 & 0 & -0.006049868 & -0.182287464 & -0.165541437 \\ 19.7 & 0 & -0.006110414 & -0.183174468 & -0.166419186 \\ 19.8 & 0 & -0.006170241 & -0.184058851 & -0.167294316 \\ 19.9 & 0 & -0.006229353 & -0.184940627 & -0.16816684 \\ 20 & 0 & -0.006287752 & -0.185819811 & -0.169036772\end{array}$

The Gibbs free energy difference of the four polytypes of boron nitride ( $\mathrm{r}-\mathrm{BN}, \mathrm{h}-\mathrm{BN}, \mathrm{c}-\mathrm{BN}$, and w-BN) as a function of pressure ranging from 0 to $20 \mathrm{GPa}$, calculated with respect to $\mathrm{r}-\mathrm{BN}$ as a reference at a given temperature of 1900 Kelvin. The unit of the Gibbs free energy difference for the four polytypes of boron nitride is given in $\mathrm{eV} /$ atom.

\begin{tabular}{lllll}
\multicolumn{2}{l}{$\mathbf{P}(\mathbf{G P a}) \mathbf{r}-\mathbf{B N}$} & $\mathbf{h}-\mathbf{B N}$ & $\mathbf{c}-\mathbf{B N}$ & $\mathbf{w}-\mathbf{B N}$ \\
0 & 0 & 0.027151974 & 0.080336469 & 0.095111895 \\
0.1 & 0 & 0.026864004 & 0.078224085 & 0.093008744 \\
0.2 & 0 & 0.026574858 & 0.07612843 & 0.090922324 \\
0.3 & 0 & 0.026284841 & 0.074049152 & 0.088852282 \\
0.4 & 0 & 0.025994228 & 0.071985912 & 0.08679828 \\
0.5 & 0 & 0.025703257 & 0.069938388 & 0.084759994 \\
0.6 & 0 & 0.025412144 & 0.067906266 & 0.082737111 \\
0.7 & 0 & 0.025121079 & 0.065889245 & 0.080729331 \\
0.8 & 0 & 0.024830233 & 0.063887036 & 0.078736364 \\
0.9 & 0 & 0.024539757 & 0.061899359 & 0.07675793 \\
1 & 0 & 0.024249787 & 0.059925945 & 0.07479376 \\
1.1 & 0 & 0.023960446 & 0.057966533 & 0.072843594
\end{tabular}




\begin{tabular}{|c|c|c|c|c|}
\hline 1.2 & 0 & 0.023671843 & 0.056020873 & 0.07090718 \\
\hline 1.3 & 0 & 0.023384077 & 0.05408872 & 0.068984275 \\
\hline 1.4 & 0 & 0.023097238 & 0.052169841 & 0.067074644 \\
\hline 1.5 & 0 & 0.022811403 & 0.050264008 & 0.06517806 \\
\hline 1.6 & 0 & 0.022526646 & 0.048371 & 0.063294303 \\
\hline 1.7 & 0 & 0.022243031 & 0.046490603 & 0.061423158 \\
\hline 1.8 & 0 & 0.021960616 & 0.044622612 & 0.059564419 \\
\hline 1.9 & 0 & 0.021679454 & 0.042766826 & 0.057717886 \\
\hline 2 & 0 & 0.021399591 & 0.040923049 & 0.055883364 \\
\hline 2.1 & 0 & 0.02112107 & 0.039091093 & 0.054060663 \\
\hline 2.2 & 0 & 0.020843929 & 0.037270775 & 0.052249601 \\
\hline 2.3 & 0 & 0.020568203 & 0.035461916 & 0.050449999 \\
\hline 2.4 & 0 & 0.020293921 & 0.033664342 & 0.048661684 \\
\hline 2.5 & 0 & 0.020021111 & 0.031877886 & 0.046884487 \\
\hline 2.6 & 0 & 0.019749798 & 0.030102384 & 0.045118244 \\
\hline 2.7 & 0 & 0.019480003 & 0.028337676 & 0.043362797 \\
\hline 2.8 & 0 & 0.019211745 & 0.026583606 & 0.041617989 \\
\hline 2.9 & 0 & 0.018945042 & 0.024840024 & 0.039883669 \\
\hline 3 & 0 & 0.018679907 & 0.023106782 & 0.038159691 \\
\hline 3.1 & 0 & 0.018416354 & 0.021383737 & 0.036445909 \\
\hline 3.2 & 0 & 0.018154395 & 0.019670749 & 0.034742186 \\
\hline 3.3 & 0 & 0.017894038 & 0.017967681 & 0.033048383 \\
\hline 3.4 & 0 & 0.017635293 & 0.0162744 & 0.031364369 \\
\hline 3.5 & 0 & 0.017378165 & 0.014590776 & 0.029690012 \\
\hline 3.6 & 0 & 0.017122661 & 0.012916682 & 0.028025187 \\
\hline 3.7 & 0 & 0.016868784 & 0.011251996 & 0.026369769 \\
\hline 3.8 & 0 & 0.016616539 & 0.009596595 & 0.024723637 \\
\hline 3.9 & 0 & 0.016365928 & 0.007950362 & 0.023086674 \\
\hline 4 & 0 & 0.016116952 & 0.006313181 & 0.021458763 \\
\hline 4.1 & 0 & 0.015869612 & 0.004684939 & 0.019839793 \\
\hline 4.2 & 0 & 0.015623909 & 0.003065526 & 0.018229652 \\
\hline 4.3 & 0 & 0.01537984 & 0.001454835 & 0.016628234 \\
\hline
\end{tabular}




\begin{tabular}{|c|c|c|c|c|}
\hline & 0 & 0.015137406 & -0.000147241 & 0.015035431 \\
\hline 4.5 & 0 & 0.014896605 & -0.001740804 & 0.013451142 \\
\hline 4.6 & 0 & 0.014657433 & -0.003325955 & 0.011875265 \\
\hline 47 & & 0.014419888 & -0.004902794 & 0.010307701 \\
\hline 4.8 & 0 & 0.014183967 & -0.006471418 & 0.008748354 \\
\hline 4.9 & 0 & 0.013949665 & -0.00803192 & 0.007197128 \\
\hline 5 & 0 & 0.013716979 & -0.009584394 & 0.005653931 \\
\hline 5.1 & 0 & 0.013485904 & -0.011128931 & 0.004118671 \\
\hline 5.2 & 0 & 0.013256436 & -0.012665621 & 0.00259126 \\
\hline 5.3 & 0 & 0.013028568 & -0.014194549 & 0.00107161 \\
\hline 5.4 & 0 & 0.012802297 & -0.015715803 & -0.000440365 \\
\hline 5.5 & 0 & 0.012577615 & -0.017229466 & -0.001944749 \\
\hline 5.6 & 0 & 0.012354517 & -0.018735621 & -0.003441623 \\
\hline 5.7 & 0 & 0.012132997 & -0.020234348 & -0.004931069 \\
\hline 5.8 & 0 & 0.011913049 & -0.021725726 & -0.006413167 \\
\hline 5.9 & 0 & 0.011694666 & -0.023209834 & -0.007887993 \\
\hline 6 & 0 & 0.011477842 & -0.024686747 & -0.009355625 \\
\hline 6.1 & 0 & 0.01126257 & -0.026156542 & -0.010816137 \\
\hline 6.2 & 0 & 0.011048842 & -0.02761929 & -0.012269602 \\
\hline 6.3 & 0 & 0.010836653 & -0.029075065 & -0.013716094 \\
\hline 6.4 & 0 & 0.010625994 & -0.030523937 & -0.015155682 \\
\hline 6.5 & 0 & 0.010416859 & -0.031965976 & -0.016588438 \\
\hline 6.6 & 0 & 0.01020924 & -0.033401251 & -0.018014428 \\
\hline 6.7 & 0 & 0.01000313 & -0.034829828 & -0.019433721 \\
\hline 6.8 & 0 & 0.009798522 & -0.036251774 & -0.020846382 \\
\hline 6.9 & 0 & 0.009595408 & -0.037667154 & -0.022252476 \\
\hline 7 & 0 & 0.00939378 & -0.039076032 & -0.023652068 \\
\hline 7.1 & 0 & 0.009193631 & -0.04047847 & -0.025045219 \\
\hline 7.2 & 0 & 0.008994953 & -0.041874529 & -0.026431993 \\
\hline 7.3 & 0 & 0.00879774 & -0.043264272 & -0.027812448 \\
\hline 7.4 & 0 & 0.008601982 & -0.044647756 & -0.029186646 \\
\hline 7.5 & 0 & 0.008407672 & -0.046025042 & -0.030554644 \\
\hline
\end{tabular}




\begin{tabular}{|c|c|c|c|c|}
\hline 7.6 & 0 & 0.008214804 & -0.047396187 & -0.031916501 \\
\hline 7.7 & 0 & 0.008023368 & -0.048761247 & -0.033272274 \\
\hline 7.8 & 0 & 0.007833357 & -0.050120278 & -0.034622017 \\
\hline 7.9 & 0 & 0.007644765 & -0.051473336 & -0.035965787 \\
\hline 8 & 0 & 0.007457582 & -0.052820475 & -0.037303637 \\
\hline 8.1 & 0 & 0.007271801 & -0.054161748 & -0.038635621 \\
\hline 8.2 & 0 & 0.007087415 & -0.055497207 & -0.039961791 \\
\hline 8.3 & 0 & 0.006904416 & -0.056826904 & -0.041282198 \\
\hline 8.4 & 0 & 0.006722796 & -0.05815089 & -0.042596895 \\
\hline 8.5 & 0 & 0.006542547 & -0.059469215 & -0.04390593 \\
\hline 8.6 & 0 & 0.006363663 & -0.060781928 & -0.045209354 \\
\hline 8.7 & 0 & 0.006186135 & -0.062089078 & -0.046507215 \\
\hline 8.8 & 0 & 0.006009957 & -0.063390714 & -0.04779956 \\
\hline 8.9 & 0 & 0.005835119 & -0.064686881 & -0.049086437 \\
\hline 9 & 0 & 0.005661616 & -0.065977627 & -0.050367892 \\
\hline 9.1 & 0 & 0.005489439 & -0.067262996 & -0.051643972 \\
\hline 9.2 & 0 & 0.005318581 & -0.068543036 & -0.052914721 \\
\hline 9.3 & 0 & 0.005149035 & -0.06981779 & -0.054180183 \\
\hline 9.4 & 0 & 0.004980793 & -0.071087301 & -0.055440404 \\
\hline 9.5 & 0 & 0.004813849 & -0.072351613 & -0.056695426 \\
\hline 9.6 & 0 & 0.004648194 & -0.073610769 & -0.057945291 \\
\hline 9.7 & 0 & 0.004483821 & -0.074864811 & -0.059190042 \\
\hline 9.8 & 0 & 0.004320724 & -0.07611378 & -0.06042972 \\
\hline 9.9 & 0 & 0.004158895 & -0.077357717 & -0.061664366 \\
\hline 10 & 0 & 0.003998327 & -0.078596663 & -0.06289402 \\
\hline 10.1 & 0 & 0.003839014 & -0.079830656 & -0.064118722 \\
\hline 10.2 & 0 & 0.003680947 & -0.081059738 & -0.065338512 \\
\hline 10.3 & 0 & 0.00352412 & -0.082283945 & -0.066553428 \\
\hline 10.4 & 0 & 0.003368526 & -0.083503316 & -0.067763509 \\
\hline 10.5 & 0 & 0.003214159 & -0.08471789 & -0.068968791 \\
\hline 10.6 & 0 & 0.00306101 & -0.085927702 & -0.070169313 \\
\hline 10.7 & 0 & 0.002909074 & -0.087132791 & -0.07136511 \\
\hline
\end{tabular}




\begin{tabular}{|c|c|c|c|c|}
\hline 10.8 & 0 & 0.002758344 & -0.088333191 & -0.072556219 \\
\hline 10.9 & 0 & 0.002608813 & -0.08952894 & -0.073742677 \\
\hline 11 & 0 & 0.002460475 & -0.090720071 & -0.074924517 \\
\hline 11.1 & 0 & 0.002313322 & -0.091906621 & -0.076101776 \\
\hline 11.2 & 0 & 0.002167348 & -0.093088623 & -0.077274487 \\
\hline 11.3 & 0 & 0.002022547 & -0.094266111 & -0.078442685 \\
\hline 11.4 & 0 & 0.001878912 & -0.095439119 & -0.079606402 \\
\hline 11.5 & 0 & 0.001736437 & -0.09660768 & -0.080765673 \\
\hline 11.6 & 0 & 0.001595115 & -0.097771827 & -0.081920529 \\
\hline 11.7 & 0 & 0.00145494 & -0.098931591 & -0.083071003 \\
\hline 11.8 & 0 & 0.001315906 & -0.100087006 & -0.084217128 \\
\hline 11.9 & 0 & 0.001178006 & -0.101238102 & -0.085358934 \\
\hline 12 & 0 & 0.001041234 & -0.102384911 & -0.086496452 \\
\hline 12.1 & 0 & 0.000905584 & -0.103527462 & -0.087629714 \\
\hline 12.2 & 0 & 0.000771051 & -0.104665788 & -0.08875875 \\
\hline 12.3 & 0 & 0.000637627 & -0.105799917 & -0.08988359 \\
\hline 12.4 & 0 & 0.000505307 & -0.106929879 & -0.091004263 \\
\hline 12.5 & 0 & 0.000374084 & -0.108055704 & -0.092120799 \\
\hline 12.6 & 0 & 0.000243954 & -0.109177421 & -0.093233227 \\
\hline 12.7 & 0 & 0.00011491 & -0.110295058 & -0.094341575 \\
\hline 12.8 & 0 & -0.0000130545 & -0.111408643 & -0.095445872 \\
\hline 12.9 & 0 & -0.000139944 & -0.112518205 & -0.096546146 \\
\hline 13 & 0 & -0.000265766 & -0.113623772 & -0.097642424 \\
\hline 13.1 & 0 & -0.000390524 & -0.114725369 & -0.098734734 \\
\hline 13.2 & 0 & -0.000514225 & -0.115823025 & -0.099823102 \\
\hline 13.3 & 0 & -0.000636875 & -0.116916767 & -0.100907556 \\
\hline 13.4 & 0 & -0.000758479 & -0.11800662 & -0.101988122 \\
\hline 13.5 & 0 & -0.000879042 & -0.119092611 & -0.103064826 \\
\hline 13.6 & 0 & -0.00099857 & -0.120174765 & -0.104137694 \\
\hline 13.7 & 0 & -0.001117069 & -0.121253108 & -0.105206752 \\
\hline 13.8 & 0 & -0.001234544 & -0.122327666 & -0.106272024 \\
\hline 13.9 & 0 & -0.001351 & -0.123398464 & -0.107333535 \\
\hline
\end{tabular}




\begin{tabular}{|c|c|c|c|c|}
\hline 14 & 0 & -0.001466443 & -0.124465525 & -0.108391311 \\
\hline 14.1 & 0 & -0.001580878 & -0.125528875 & -0.109445376 \\
\hline 14.2 & 0 & -0.00169431 & -0.126588537 & -0.110495754 \\
\hline 14.3 & 0 & -0.001806745 & -0.127644536 & -0.111542468 \\
\hline 14.4 & 0 & -0.001918187 & -0.128696896 & -0.112585544 \\
\hline 14.5 & 0 & -0.002028642 & -0.129745639 & -0.113625003 \\
\hline 14.6 & 0 & -0.002138115 & -0.130790788 & -0.114660869 \\
\hline 14.7 & 0 & -0.002246611 & -0.131832368 & -0.115693166 \\
\hline 14.8 & 0 & -0.002354134 & -0.1328704 & -0.116721915 \\
\hline 14.9 & 0 & -0.002460691 & -0.133904906 & -0.11774714 \\
\hline 15 & 0 & -0.002566285 & -0.13493591 & -0.118768861 \\
\hline 15.1 & 0 & -0.002670922 & -0.135963433 & -0.119787103 \\
\hline 15.2 & 0 & -0.002774607 & -0.136987496 & -0.120801885 \\
\hline 15.3 & 0 & -0.002877344 & -0.138008122 & -0.12181323 \\
\hline 15.4 & 0 & -0.002979139 & -0.139025331 & -0.122821159 \\
\hline 15.5 & 0 & -0.003079995 & -0.140039145 & -0.123825693 \\
\hline 15.6 & 0 & -0.003179918 & -0.141049584 & -0.124826853 \\
\hline 15.7 & 0 & -0.003278913 & -0.14205667 & -0.125824659 \\
\hline 15.8 & 0 & -0.003376983 & -0.143060422 & -0.126819133 \\
\hline 15.9 & 0 & -0.003474135 & -0.144060861 & -0.127810294 \\
\hline 16 & 0 & -0.003570371 & -0.145058007 & -0.128798162 \\
\hline 16.1 & 0 & -0.003665697 & -0.14605188 & -0.129782757 \\
\hline 16.2 & 0 & -0.003760118 & -0.147042498 & -0.1307641 \\
\hline 16.3 & 0 & -0.003853637 & -0.148029883 & -0.131742208 \\
\hline 16.4 & 0 & -0.003946259 & -0.149014053 & -0.132717102 \\
\hline 16.5 & 0 & -0.004037989 & -0.149995028 & -0.133688801 \\
\hline 16.6 & 0 & -0.004128831 & -0.150972825 & -0.134657324 \\
\hline 16.7 & 0 & -0.00421879 & -0.151947464 & -0.135622688 \\
\hline 16.8 & 0 & -0.004307869 & -0.152918963 & -0.136584914 \\
\hline 16.9 & 0 & -0.004396072 & -0.153887342 & -0.137544019 \\
\hline 17 & 0 & -0.004483405 & -0.154852617 & -0.138500021 \\
\hline 7.1 & 0 & -0.004569872 & -0.155814806 & -0.139452939 \\
\hline
\end{tabular}




\begin{tabular}{lllll}
17.2 & 0 & -0.004655475 & -0.156773929 & -0.14040279 \\
17.3 & 0 & -0.004740221 & -0.157730002 & -0.141349591 \\
17.4 & 0 & -0.004824112 & -0.158683043 & -0.142293362 \\
17.5 & 0 & -0.004907154 & -0.159633069 & -0.143234118 \\
17.6 & 0 & -0.004989349 & -0.160580098 & -0.144171877 \\
17.7 & 0 & -0.005070702 & -0.161524146 & -0.145106656 \\
17.8 & 0 & -0.005151217 & -0.16246523 & -0.146038471 \\
17.9 & 0 & -0.005230897 & -0.163403367 & -0.146967341 \\
18 & 0 & -0.005309748 & -0.164338574 & -0.147893281 \\
18.1 & 0 & -0.005387773 & -0.165270867 & -0.148816307 \\
18.2 & 0 & -0.005464975 & -0.166200262 & -0.149736436 \\
18.3 & 0 & -0.005541359 & -0.167126776 & -0.150653684 \\
18.4 & 0 & -0.005616928 & -0.168050424 & -0.151568067 \\
18.5 & 0 & -0.005691686 & -0.168971221 & -0.1524796 \\
18.6 & 0 & -0.005765637 & -0.169889185 & -0.1533883 \\
18.7 & 0 & -0.005838784 & -0.17080433 & -0.154294182 \\
18.8 & 0 & -0.005911132 & -0.171716672 & -0.155197262 \\
18.9 & 0 & -0.005982684 & -0.172626226 & -0.156097554 \\
19 & 0 & -0.006053444 & -0.173533007 & -0.156995074 \\
19.1 & 0 & -0.006123415 & -0.17443703 & -0.157889836 \\
19.2 & 0 & -0.006192601 & -0.17533831 & -0.158781857 \\
19.3 & 0 & -0.006261005 & -0.176236862 & -0.159671149 \\
19.4 & 0 & -0.006328632 & -0.1771327 & -0.160557729 \\
19.5 & 0 & -0.006395484 & -0.178025839 & -0.161441611 \\
19.6 & 0 & -0.006461565 & -0.178916294 & -0.162322808 \\
\hline 19.9 & 0 & -0.006526878 & -0.179804078 & -0.163201335 \\
\hline 10 & 0 & -0.006718249 & -0.182451546 & -0.165821039
\end{tabular}


The Gibbs free energy difference of the four polytypes of boron nitride ( $\mathrm{r}-\mathrm{BN}, \mathrm{h}-\mathrm{BN}, \mathrm{c}-\mathrm{BN}$, and w-BN) as a function of pressure ranging from 0 to $20 \mathrm{GPa}$, calculated with respect to $\mathrm{r}-\mathrm{BN}$ as a reference at a given temperature of $\mathbf{2 0 0 0}$ Kelvin. The unit of the Gibbs free energy difference for the four polytypes of boron nitride is given in $\mathrm{eV} /$ atom.

\begin{tabular}{|c|c|c|c|c|}
\hline \multicolumn{2}{|c|}{$\mathrm{P}(\mathrm{GPa}) \mathrm{r}-\mathrm{BN}$} & \multirow{2}{*}{$\begin{array}{l}\text { h-BN } \\
0.028560974\end{array}$} & \multirow{2}{*}{$\begin{array}{l}\text { c-BN } \\
0.084437635\end{array}$} & \multirow{2}{*}{$\begin{array}{l}\text { w-BN } \\
0.099068993\end{array}$} \\
\hline 0 & 0 & & & \\
\hline 0.1 & 0 & 0.028264942 & 0.082324661 & 0.096965215 \\
\hline 0.2 & 0 & 0.02796742 & 0.080228047 & 0.094877797 \\
\hline 0.3 & 0 & 0.027668738 & 0.078147464 & 0.092806412 \\
\hline 0.4 & 0 & 0.027369185 & 0.076082596 & 0.090750742 \\
\hline 0.5 & 0 & 0.027069021 & 0.074033138 & 0.088710484 \\
\hline 0.6 & 0 & 0.026768477 & 0.071998796 & 0.086685344 \\
\hline 0.7 & 0 & 0.026467758 & 0.069979288 & 0.084675038 \\
\hline 0.8 & 0 & 0.02616705 & 0.067974339 & 0.082679293 \\
\hline 0.9 & 0 & 0.02586652 & 0.065983687 & 0.080697845 \\
\hline 1 & 0 & 0.025566315 & 0.064007076 & 0.078730439 \\
\hline 1.1 & 0 & 0.025266571 & 0.062044259 & 0.076776829 \\
\hline 1.2 & 0 & 0.024967408 & 0.060094998 & 0.074836775 \\
\hline 1.3 & 0 & 0.024668937 & 0.058159061 & 0.072910047 \\
\hline 1.4 & 0 & 0.024371256 & 0.056236225 & 0.070996421 \\
\hline 1.5 & 0 & 0.024074455 & 0.054326274 & 0.06909568 \\
\hline 1.6 & 0 & 0.023778614 & 0.052428996 & 0.067207614 \\
\hline 1.7 & 0 & 0.023483807 & 0.050544188 & 0.065332019 \\
\hline 1.8 & 0 & 0.023190101 & 0.048671652 & 0.063468697 \\
\hline 1.9 & 0 & 0.022897555 & 0.046811197 & 0.061617456 \\
\hline 2 & 0 & 0.022606223 & 0.044962634 & 0.059778109 \\
\hline 2.1 & 0 & 0.022316155 & 0.043125784 & 0.057950475 \\
\hline 2.2 & 0 & 0.022027396 & 0.041300469 & 0.056134378 \\
\hline 2.3 & 0 & 0.021739986 & 0.039486518 & 0.054329646 \\
\hline 2.4 & 0 & 0.021453961 & 0.037683765 & 0.052536112 \\
\hline 2.5 & 0 & 0.021169354 & 0.035892047 & 0.050753613 \\
\hline 2.6 & 0 & 0.020886194 & 0.034111205 & 0.048981993 \\
\hline
\end{tabular}




\begin{tabular}{|c|c|c|c|c|}
\hline 2.7 & 0 & 0.020604509 & 0.032341087 & 0.047221096 \\
\hline 2.8 & 0 & 0.020324323 & 0.030581541 & 0.045470773 \\
\hline 2.9 & 0 & 0.020045656 & 0.028832422 & 0.043730877 \\
\hline 3 & 0 & 0.019768528 & 0.027093587 & 0.042001267 \\
\hline 3.1 & 0 & 0.019492956 & 0.025364897 & 0.040281802 \\
\hline 3.2 & 0 & 0.019218955 & 0.023646216 & 0.038572347 \\
\hline 3.3 & 0 & 0.018946538 & 0.021937412 & 0.036872769 \\
\hline 3.4 & 0 & 0.018675717 & 0.020238356 & 0.03518294 \\
\hline 3.5 & 0 & 0.018406502 & 0.018548921 & 0.033502734 \\
\hline 3.6 & 0 & 0.018138903 & 0.016868984 & 0.031832026 \\
\hline 3.7 & 0 & 0.017872925 & 0.015198425 & 0.030170696 \\
\hline 3.8 & 0 & 0.017608576 & 0.013537126 & 0.028518627 \\
\hline 3.9 & 0 & 0.017345861 & 0.011884972 & 0.026875704 \\
\hline 4 & 0 & 0.017084784 & 0.01024185 & 0.025241814 \\
\hline 4.1 & 0 & 0.016825349 & 0.008607651 & 0.023616847 \\
\hline 4.2 & 0 & 0.016567557 & 0.006982267 & 0.022000696 \\
\hline 4.3 & 0 & 0.01631141 & 0.005365592 & 0.020393254 \\
\hline 4.4 & 0 & 0.016056909 & 0.003757523 & 0.01879442 \\
\hline 4.5 & 0 & 0.015804055 & 0.002157959 & 0.017204091 \\
\hline 4.6 & 0 & 0.015552847 & 0.000566802 & 0.015622169 \\
\hline 4.7 & 0 & 0.015303283 & -0.001016045 & 0.014048558 \\
\hline 4.8 & 0 & 0.015055362 & -0.002590677 & 0.012483163 \\
\hline 4.9 & 0 & 0.014809083 & -0.004157187 & 0.01092589 \\
\hline 5 & 0 & 0.014564442 & -0.005715666 & 0.009376649 \\
\hline 5.1 & 0 & 0.014321436 & -0.007266204 & 0.00783535 \\
\hline 5.2 & 0 & 0.014080063 & -0.008808887 & 0.006301906 \\
\hline 5.3 & 0 & 0.013840318 & -0.010343801 & 0.004776231 \\
\hline 5.4 & 0 & 0.013602197 & -0.011871031 & 0.003258241 \\
\hline 5.5 & 0 & 0.013365697 & -0.013390659 & 0.001747853 \\
\hline 5.6 & 0 & 0.013130811 & -0.014902767 & 0.000244987 \\
\hline 5.7 & 0 & 0.012897536 & -0.016407433 & -0.001250438 \\
\hline 5.8 & 0 & 0.012665866 & -0.017904735 & -0.002738498 \\
\hline
\end{tabular}




\begin{tabular}{|c|c|c|c|c|}
\hline 5.9 & 0 & 0.012435796 & -0.019394751 & -0.004219271 \\
\hline 6 & 0 & 0.012207321 & -0.020877554 & -0.005692832 \\
\hline 6.1 & 0 & 0.011980433 & -0.02235322 & -0.007159255 \\
\hline 6.2 & 0 & 0.011755128 & -0.023821821 & -0.008618611 \\
\hline 6.3 & 0 & 0.011531399 & -0.025283426 & -0.010070973 \\
\hline 6.4 & 0 & 0.01130924 & -0.026738108 & -0.01151641 \\
\hline 6.5 & 0 & 0.011088644 & -0.028185934 & -0.012954991 \\
\hline 6.6 & 0 & 0.010869606 & -0.029626972 & -0.014386783 \\
\hline 6.7 & 0 & 0.010652118 & -0.031061288 & -0.015811854 \\
\hline 6.8 & 0 & 0.010436173 & -0.032488948 & -0.017230268 \\
\hline 6.9 & 0 & 0.010221766 & -0.033910015 & -0.018642088 \\
\hline 7 & 0 & 0.010008888 & -0.035324553 & -0.02004738 \\
\hline 7.1 & 0 & 0.009797533 & -0.036732624 & -0.021446203 \\
\hline 7.2 & 0 & 0.009587695 & -0.038134288 & -0.02283862 \\
\hline 7.3 & 0 & 0.009379365 & -0.039529607 & -0.024224691 \\
\hline 7.4 & 0 & 0.009172536 & -0.040918638 & -0.025604474 \\
\hline 7.5 & 0 & 0.008967203 & -0.04230144 & -0.026978027 \\
\hline 7.6 & 0 & 0.008763356 & -0.04367807 & -0.028345408 \\
\hline 7.7 & 0 & 0.00856099 & -0.045048584 & -0.029706673 \\
\hline 7.8 & 0 & 0.008360096 & -0.046413038 & -0.031061878 \\
\hline 7.9 & 0 & 0.008160668 & -0.047771486 & -0.032411077 \\
\hline 8 & 0 & 0.007962698 & -0.049123981 & -0.033754323 \\
\hline 8.1 & 0 & 0.007766179 & -0.050470578 & -0.035091669 \\
\hline 8.2 & 0 & 0.007571103 & -0.051811327 & -0.036423168 \\
\hline 8.3 & 0 & 0.007377464 & -0.05314628 & -0.03774887 \\
\hline 8.4 & 0 & 0.007185253 & -0.054475487 & -0.039068827 \\
\hline 8.5 & 0 & 0.006994464 & -0.055798998 & -0.040383087 \\
\hline 8.6 & 0 & 0.006805089 & -0.057116863 & -0.041691701 \\
\hline 8.7 & 0 & 0.006617121 & -0.058429128 & -0.042994715 \\
\hline 8.8 & 0 & 0.006430552 & -0.059735843 & -0.044292178 \\
\hline 8.9 & 0 & 0.006245376 & -0.061037053 & -0.045584137 \\
\hline 9 & 0 & 0.006061584 & -0.062332805 & -0.046870638 \\
\hline
\end{tabular}




\begin{tabular}{|c|c|c|c|c|}
\hline .1 & 0 & 0.005879171 & -0.063623145 & -0.048151725 \\
\hline 9.2 & 0 & 0.005698128 & -0.064908117 & -0.049427445 \\
\hline 9.3 & 0 & 0.005518448 & -0.066187765 & -0.050697841 \\
\hline 9.4 & & 0.005340124 & -0.067462133 & -0.051962958 \\
\hline 9.5 & 0 & 0.00516315 & -0.068731265 & -0.053222837 \\
\hline 9.6 & 0 & 0.004987516 & -0.069995202 & -0.054477522 \\
\hline 9.7 & 0 & 0.004813218 & -0.071253987 & -0.055727054 \\
\hline 9.8 & 0 & 0.004640248 & -0.07250766 & -0.056971475 \\
\hline 9.9 & 0 & 0.004468598 & -0.073756262 & -0.058210824 \\
\hline 10 & 0 & 0.004298261 & -0.074999835 & -0.059445144 \\
\hline 10.1 & 0 & 0.004129231 & -0.076238416 & -0.060674472 \\
\hline 10.2 & 0 & 0.003961501 & -0.077472045 & -0.061898849 \\
\hline 10.3 & 0 & 0.003795063 & -0.078700761 & -0.063118312 \\
\hline 10.4 & 0 & 0.003629911 & -0.079924602 & -0.0643329 \\
\hline 10.5 & 0 & 0.003466038 & -0.081143605 & -0.065542651 \\
\hline 10.6 & 0 & 0.003303438 & -0.082357808 & -0.0667476 \\
\hline 10.7 & 0 & 0.003142103 & -0.083567247 & -0.067947786 \\
\hline 10.8 & 0 & 0.002982026 & -0.084771957 & -0.069143244 \\
\hline 10.9 & 0 & 0.002823202 & -0.085971976 & -0.07033401 \\
\hline 11 & 0 & 0.002665623 & -0.087167337 & -0.071520118 \\
\hline 11.1 & 0 & 0.002509283 & -0.088358076 & -0.072701605 \\
\hline 11.2 & 0 & 0.002354175 & -0.089544228 & -0.073878503 \\
\hline 11.3 & 0 & 0.002200293 & -0.090725825 & -0.075050848 \\
\hline 11.4 & 0 & 0.002047629 & -0.091902902 & -0.076218672 \\
\hline 11.5 & 0 & 0.001896179 & -0.093075491 & -0.077382008 \\
\hline 11.6 & 0 & 0.001745935 & -0.094243625 & -0.07854089 \\
\hline 11.7 & 0 & 0.001596891 & -0.095407336 & -0.079695349 \\
\hline 11.8 & 0 & 0.00144904 & -0.096566657 & -0.080845418 \\
\hline 11.9 & 0 & 0.001302377 & -0.097721619 & -0.081991127 \\
\hline 12 & 0 & 0.001156895 & -0.098872252 & -0.083132508 \\
\hline 12.1 & 0 & 0.001012588 & -0.100018588 & -0.084269592 \\
\hline 12.2 & 0 & 0.00086945 & -0.101160657 & -0.085402409 \\
\hline
\end{tabular}




\begin{tabular}{|c|c|c|c|c|}
\hline 12.3 & 0 & 0.000727474 & -0.102298488 & -0.086530989 \\
\hline 12.4 & 0 & 0.000586655 & -0.103432113 & -0.087655362 \\
\hline 12.5 & 0 & 0.000446987 & -0.104561559 & -0.088775557 \\
\hline 12.6 & 0 & 0.000308463 & -0.105686856 & -0.089891603 \\
\hline 12.7 & 0 & 0.000171077 & -0.106808032 & -0.091003528 \\
\hline 12.8 & 0 & 0.0000348245 & -0.107925116 & -0.092111361 \\
\hline 12.9 & 0 & -0.000100301 & -0.109038137 & -0.093215131 \\
\hline 13 & 0 & -0.000234306 & -0.11014712 & -0.094314864 \\
\hline 13.1 & 0 & -0.000367196 & -0.111252094 & -0.095410587 \\
\hline 13.2 & 0 & -0.000498976 & -0.112353086 & -0.096502329 \\
\hline 13.3 & 0 & -0.000629653 & -0.113450123 & -0.097590116 \\
\hline 13.4 & 0 & -0.000759231 & -0.11454323 & -0.098673974 \\
\hline 13.5 & 0 & -0.000887717 & -0.115632435 & -0.099753929 \\
\hline 13.6 & P & -0.001015117 & -0.116717762 & -0.100830007 \\
\hline 13.7 & 0 & -0.001141435 & -0.117799239 & -0.101902234 \\
\hline 13.8 & 0 & -0.001266677 & -0.118876889 & -0.102970636 \\
\hline 13.9 & 0 & -0.001390848 & -0.119950738 & -0.104035236 \\
\hline 14 & 0 & -0.001513955 & -0.12102081 & -0.10509606 \\
\hline 14.1 & 0 & -0.001636003 & -0.122087131 & -0.106153133 \\
\hline 14.2 & 0 & -0.001756996 & -0.123149724 & -0.107206479 \\
\hline 14.3 & 0 & -0.00187694 & -0.124208613 & -0.10825612 \\
\hline 14.4 & 0 & -0.00199584 & -0.125263822 & -0.109302083 \\
\hline 14.5 & 0 & -0.002113703 & -0.126315375 & -0.110344389 \\
\hline 14.6 & 0 & -0.002230532 & -0.127363295 & -0.111383061 \\
\hline 14.7 & 0 & -0.002346333 & -0.128407603 & -0.112418124 \\
\hline 14.8 & 0 & -0.002461111 & -0.129448325 & -0.1134496 \\
\hline 14.9 & 0 & -0.002574872 & -0.130485481 & -0.114477511 \\
\hline 15 & 0 & -0.00268762 & -0.131519095 & -0.115501879 \\
\hline 15.1 & 0 & -0.00279936 & -0.132549187 & -0.116522727 \\
\hline 15.2 & 0 & -0.002910097 & -0.133575781 & -0.117540076 \\
\hline 15.3 & 0 & -0.003019836 & -0.134598897 & -0.118553947 \\
\hline 15.4 & 0 & -0.003128583 & -0.135618557 & -0.119564364 \\
\hline
\end{tabular}




\begin{tabular}{|c|c|c|c|c|}
\hline 15.5 & 0 & -0.003236341 & -0.136634782 & -0.120571345 \\
\hline 15.6 & 0 & -0.003343116 & -0.137647593 & -0.121574913 \\
\hline 15.7 & 0 & -0.003448913 & -0.138657011 & -0.122575088 \\
\hline 15.8 & 0 & -0.003553736 & -0.139663056 & -0.123571891 \\
\hline 15.9 & 0 & -0.003657591 & -0.140665748 & -0.124565342 \\
\hline 16 & 0 & -0.00376048 & -0.141665109 & -0.125555461 \\
\hline 16.1 & 0 & -0.003862411 & -0.142661157 & -0.126542268 \\
\hline 16.2 & 0 & -0.003963386 & -0.143653912 & -0.127525782 \\
\hline 16.3 & 0 & -0.004063411 & -0.144643394 & -0.128506025 \\
\hline 16.4 & 0 & -0.004162491 & -0.145629623 & -0.129483013 \\
\hline 16.5 & 0 & -0.004260628 & -0.146612617 & -0.130456768 \\
\hline 16.6 & 0 & -0.00435783 & -0.147592395 & -0.131427307 \\
\hline 16.7 & 0 & -0.004454099 & -0.148568977 & -0.13239465 \\
\hline 16.8 & 0 & -0.004549439 & -0.14954238 & $-0.13335881 \epsilon$ \\
\hline 16.9 & 0 & -0.004643857 & -0.150512624 & -0.134319822 \\
\hline 17 & 0 & -0.004737355 & -0.151479726 & -0.135277687 \\
\hline 17.1 & 0 & -0.004829939 & -0.152443704 & -0.136232429 \\
\hline 17.2 & 0 & -0.004921612 & -0.153404577 & -0.137184066 \\
\hline 17.3 & 0 & -0.005012379 & -0.154362363 & -0.138132615 \\
\hline 17.4 & 0 & -0.005102243 & -0.155317078 & -0.139078096 \\
\hline 17.5 & 0 & -0.00519121 & -0.156268741 & -0.140020524 \\
\hline 17.6 & 0 & -0.005279284 & -0.157217368 & -0.140959917 \\
\hline 17.7 & 0 & -0.005366468 & -0.158162977 & -0.141896292 \\
\hline 17.8 & 0 & -0.005452766 & -0.159105585 & -0.142829666 \\
\hline 17.9 & 0 & -0.005538184 & -0.160045208 & -0.143760057 \\
\hline 18 & 0 & -0.005622724 & -0.160981863 & -0.14468748 \\
\hline 18.1 & 0 & -0.005706391 & -0.161915566 & -0.145611952 \\
\hline 18.2 & 0 & -0.005789189 & -0.162846335 & -0.146533489 \\
\hline 18.3 & 0 & -0.005871122 & -0.163774185 & -0.147452109 \\
\hline 18.4 & 0 & -0.005952193 & -0.164699132 & -0.148367826 \\
\hline 18.5 & 0 & -0.006032407 & -0.165621192 & -0.149280656 \\
\hline 18.6 & 0 & -0.006111768 & -0.166540381 & -0.150190617 \\
\hline
\end{tabular}




$\begin{array}{lllll}18.7 & 0 & -0.00619028 & -0.167456714 & -0.151097722 \\ 18.8 & 0 & -0.006267945 & -0.168370207 & -0.152001988 \\ 18.9 & 0 & -0.006344769 & -0.169280876 & -0.152903429 \\ 19 & 0 & -0.006420755 & -0.170188736 & -0.153802062 \\ 19.1 & 0 & -0.006495906 & -0.171093801 & -0.154697902 \\ 19.2 & 0 & -0.006570227 & -0.171996087 & -0.155590962 \\ 19.3 & 0 & -0.00664372 & -0.172895608 & -0.156481259 \\ 19.4 & 0 & -0.006716391 & -0.173792379 & -0.157368806 \\ 19.5 & 0 & -0.006788242 & -0.174686416 & -0.158253619 \\ 19.6 & 0 & -0.006859277 & -0.175577731 & -0.159135712 \\ 19.7 & 0 & -0.0069295 & -0.17646634 & -0.160015099 \\ 19.8 & 0 & -0.006998914 & -0.177352257 & -0.160891794 \\ 19.9 & 0 & -0.007067523 & -0.178235496 & -0.161765812 \\ 20 & 0 & -0.00713533 & -0.179116071 & -0.162637166\end{array}$

The Gibbs free energy difference of the four polytypes of boron nitride ( $r-\mathrm{BN}, \mathrm{h}-\mathrm{BN}, \mathrm{c}-\mathrm{BN}$, and w-BN) as a function of pressure ranging from 0 to $20 \mathrm{GPa}$, calculated with respect to $\mathrm{r}-\mathrm{BN}$ as a reference at a given temperature of 2100 Kelvin. The unit of the Gibbs free energy difference for the four polytypes of boron nitride is given in $\mathrm{eV} /$ atom.

$\begin{array}{lllll}\mathbf{P}(\mathbf{G P a}) \mathbf{r}-\mathbf{B N} & \mathbf{h}-\mathbf{B N} & \mathbf{c}-\mathbf{B N} & \mathbf{w}-\mathbf{B N} \\ 0 & 0 & 0.029985965 & 0.088488008 & 0.102970207 \\ 0.1 & 0 & 0.029682474 & 0.086375144 & 0.100866521 \\ 0.2 & 0 & 0.029377169 & 0.084278262 & 0.098778818 \\ 0.3 & 0 & 0.029070398 & 0.082197055 & 0.096706792 \\ 0.4 & 0 & 0.028762471 & 0.080131228 & 0.094650147 \\ 0.5 & 0 & 0.028453664 & 0.078080495 & 0.092608598 \\ 0.6 & 0 & 0.028144224 & 0.076044582 & 0.090581869 \\ 0.7 & 0 & 0.027834373 & 0.074023221 & 0.088569695 \\ 0.8 & 0 & 0.027524312 & 0.072016157 & 0.086571817 \\ 0.9 & 0 & 0.027214219 & 0.070023139 & 0.084587988 \\ 1 & 0 & 0.026904258 & 0.068043927 & 0.082617967 \\ 1.1 & 0 & 0.026594574 & 0.066078288 & 0.080661519\end{array}$




\begin{tabular}{|c|c|c|c|c|}
\hline 1.2 & 0 & 0.026285301 & 0.064125995 & 0.078718419 \\
\hline 1.3 & 0 & 0.02597656 & 0.062186831 & 0.076788449 \\
\hline 1.4 & 0 & 0.025668458 & 0.060260581 & 0.074871395 \\
\hline 1.5 & 0 & 0.025361096 & 0.058347041 & 0.072967051 \\
\hline 1.6 & 0 & 0.025054564 & 0.05644601 & 0.071075218 \\
\hline 1.7 & 0 & 0.024748945 & 0.054557294 & 0.0691957 \\
\hline 1.8 & 0 & 0.024444311 & 0.052680703 & 0.06732831 \\
\hline 1.9 & 0 & 0.024140733 & 0.050816054 & 0.065472862 \\
\hline 2 & 0 & 0.02383827 & 0.04896317 & 0.06362918 \\
\hline 2.1 & 0 & 0.023536981 & 0.047121875 & 0.061797089 \\
\hline 2.2 & 0 & 0.023236916 & 0.045292001 & 0.059976419 \\
\hline 2.3 & 0 & 0.022938121 & 0.043473384 & 0.058167008 \\
\hline 2.4 & 0 & 0.02264064 & 0.041665863 & 0.056368694 \\
\hline 2.5 & 0 & 0.022344511 & 0.039869283 & 0.054581322 \\
\hline 2.6 & 0 & 0.022049769 & 0.03808349 & 0.052804738 \\
\hline 2.7 & 0 & 0.021756446 & 0.036308338 & 0.051038796 \\
\hline 2.8 & 0 & 0.02146457 & 0.03454368 & 0.049283349 \\
\hline 2.9 & 0 & 0.021174169 & 0.032789377 & 0.047538258 \\
\hline 3 & 0 & 0.020885265 & 0.03104529 & 0.045803384 \\
\hline 3.1 & 0 & 0.020597881 & 0.029311285 & 0.044078593 \\
\hline 3.2 & 0 & 0.020312034 & 0.027587231 & 0.042363754 \\
\hline 3.3 & 0 & 0.020027743 & 0.025873 & 0.040658739 \\
\hline 3.4 & 0 & 0.019745022 & 0.024168466 & 0.038963423 \\
\hline 3.5 & 0 & 0.019463886 & 0.022473508 & 0.037277682 \\
\hline 3.6 & 0 & 0.019184347 & 0.020788005 & 0.035601398 \\
\hline 3.7 & 0 & 0.018906415 & 0.019111841 & 0.033934454 \\
\hline 3.8 & 0 & 0.018630099 & 0.017444901 & 0.032276735 \\
\hline 3.9 & 0 & 0.018355409 & 0.015787074 & 0.030628129 \\
\hline 4 & 0 & 0.01808235 & 0.014138251 & 0.028988528 \\
\hline 4.1 & 0 & 0.017810929 & 0.012498323 & 0.027357823 \\
\hline 4.2 & 0 & 0.01754115 & 0.010867187 & 0.025735911 \\
\hline 4.3 & 0 & 0.017273019 & 0.009244739 & 0.024122688 \\
\hline
\end{tabular}




\begin{tabular}{|c|c|c|c|c|}
\hline & 0 & 0.017006537 & 0.007630879 & 0.022518054 \\
\hline 4.5 & 0 & 0.016741708 & 0.00602551 & 0.020921911 \\
\hline 4.6 & 0 & 0.016478533 & 0.004428533 & 0.019334162 \\
\hline 47 & & 0.016217012 & 0.002839856 & 0.017754712 \\
\hline 4.8 & 0 & 0.015957147 & 0.001259384 & 0.016183469 \\
\hline 4.9 & 0 & 0.015698938 & -0.000312972 & 0.014620343 \\
\hline 5 & 0 & 0.015442382 & -0.001877301 & 0.013065243 \\
\hline 5.1 & 0 & 0.01518748 & -0.003433692 & 0.011518084 \\
\hline 5.2 & 0 & 0.014934229 & -0.004982229 & 0.009978778 \\
\hline 5.3 & 0 & 0.014682628 & -0.006522997 & 0.008447242 \\
\hline 5.4 & 0 & 0.014432673 & -0.008056078 & 0.006923395 \\
\hline 5.5 & 0 & 0.014184362 & -0.009581553 & 0.005407153 \\
\hline 5.6 & 0 & 0.013937692 & -0.011099501 & 0.003898439 \\
\hline 5.7 & 0 & 0.013692658 & -0.012610001 & 0.002397175 \\
\hline 5.8 & 0 & 0.013449257 & -0.014113128 & 0.000903283 \\
\hline 5.9 & 0 & 0.013207485 & -0.015608958 & -0.000583311 \\
\hline 6 & 0 & 0.012967337 & -0.017097566 & -0.002062682 \\
\hline 6.1 & 0 & 0.012728808 & -0.018579022 & -0.003534901 \\
\hline 6.2 & 0 & 0.012491894 & -0.0200534 & -0.005000041 \\
\hline 6.3 & 0 & 0.01225659 & -0.021520768 & -0.00645817 \\
\hline 6.4 & 0 & 0.01202289 & -0.022981195 & -0.007909359 \\
\hline 6.5 & 0 & 0.011790789 & -0.02443475 & -0.009353674 \\
\hline 6.6 & 0 & 0.01156028 & -0.025881498 & -0.010791182 \\
\hline 6.7 & 0 & 0.011331359 & -0.027321506 & -0.012221949 \\
\hline 6.8 & 0 & 0.01110402 & -0.028754836 & -0.013646039 \\
\hline 6.9 & 0 & 0.010878256 & -0.030181553 & -0.015063514 \\
\hline 7 & 0 & 0.010654061 & -0.031601719 & -0.016474438 \\
\hline 7.1 & 0 & 0.010431428 & -0.033015395 & -0.017878871 \\
\hline 7.2 & 0 & 0.010210353 & -0.034422641 & -0.019276874 \\
\hline 7.3 & 0 & 0.009990828 & -0.035823516 & -0.020668506 \\
\hline 7.4 & 0 & 0.009772846 & -0.037218079 & -0.022053825 \\
\hline 7.5 & 0 & 0.009556401 & -0.038606386 & -0.023432888 \\
\hline
\end{tabular}




\begin{tabular}{|c|c|c|c|c|}
\hline 7.6 & 0 & 0.009341487 & -0.039988496 & -0.024805753 \\
\hline 7.7 & 0 & 0.009128096 & -0.041364462 & -0.026172474 \\
\hline 7.8 & 0 & 0.008916222 & -0.04273434 & -0.027533106 \\
\hline 7.9 & 0 & 0.008705858 & -0.044098184 & -0.028887704 \\
\hline 8 & 0 & 0.008496998 & -0.045456046 & -0.03023632 \\
\hline 8.1 & 0 & 0.008289634 & -0.046807979 & -0.031579007 \\
\hline 8.2 & 0 & 0.008083759 & -0.048154035 & -0.032915816 \\
\hline 8.3 & 0 & 0.007879367 & -0.049494264 & -0.034246798 \\
\hline 8.4 & 0 & 0.007676451 & -0.050828716 & -0.035572003 \\
\hline 8.5 & 0 & 0.007475003 & -0.052157441 & -0.03689148 \\
\hline 8.6 & 0 & 0.007275017 & -0.053480487 & -0.038205277 \\
\hline 8.7 & 0 & 0.007076486 & -0.054797901 & -0.039513443 \\
\hline 8.8 & 0 & 0.006879402 & -0.056109732 & -0.040816025 \\
\hline 8.9 & 0 & 0.006683759 & -0.057416025 & -0.042113069 \\
\hline 9 & 0 & 0.00648955 & -0.058716826 & -0.043404621 \\
\hline 9.1 & 0 & 0.006296768 & -0.06001218 & -0.044690725 \\
\hline 9.2 & 0 & 0.006105405 & -0.061302132 & -0.045971428 \\
\hline 9.3 & 0 & 0.005915455 & -0.062586726 & -0.047246772 \\
\hline 9.4 & 0 & 0.005726911 & -0.063866005 & -0.048516801 \\
\hline 9.5 & 0 & 0.005539766 & -0.065140011 & -0.049781557 \\
\hline 9.6 & 0 & 0.005354013 & -0.066408788 & -0.051041083 \\
\hline 9.7 & 0 & 0.005169645 & -0.067672376 & -0.052295421 \\
\hline 9.8 & 0 & 0.004986655 & -0.068930817 & -0.05354461 \\
\hline 9.9 & 0 & 0.004805036 & -0.07018415 & -0.054788692 \\
\hline 10 & 0 & 0.004624782 & -0.071432416 & -0.056027707 \\
\hline 10.1 & 0 & 0.004445885 & -0.072675654 & -0.057261694 \\
\hline 10.2 & 0 & 0.00426834 & -0.073913904 & -0.058490692 \\
\hline 10.3 & 0 & 0.004092138 & -0.075147202 & -0.059714739 \\
\hline 10.4 & 0 & 0.003917273 & -0.076375588 & -0.060933874 \\
\hline 10.5 & 0 & 0.003743739 & -0.077599098 & -0.062148132 \\
\hline 10.6 & 0 & 0.00357153 & -0.07881777 & -0.063357552 \\
\hline 10.7 & 0 & 0.003400636 & -0.08003164 & -0.06456217 \\
\hline
\end{tabular}




\begin{tabular}{|c|c|c|c|c|}
\hline 10.8 & 0 & 0.003231054 & -0.081240744 & -0.065762022 \\
\hline 10.9 & 0 & 0.003062776 & -0.082445117 & -0.066957143 \\
\hline 11 & 0 & 0.002895794 & -0.083644794 & -0.068147568 \\
\hline 11.1 & 0 & 0.002730104 & -0.084839811 & -0.069333332 \\
\hline 11.2 & 0 & 0.002565698 & -0.0860302 & -0.07051447 \\
\hline 1.3 & 0 & 0.00240257 & -0.087215997 & -0.071691014 \\
\hline 11.4 & 0 & 0.002240713 & -0.088397234 & -0.072862999 \\
\hline 11.5 & 0 & 0.002080121 & -0.089573944 & -0.074030457 \\
\hline 11.6 & 0 & 0.001920788 & -0.090746161 & -0.075193422 \\
\hline 11.7 & 0 & 0.001762707 & -0.091913915 & -0.076351923 \\
\hline 11.8 & 0 & 0.001605872 & -0.093077239 & -0.077505995 \\
\hline 11.9 & 0 & 0.001450276 & -0.094236164 & -0.078655668 \\
\hline 12 & 0 & 0.001295914 & -0.095390722 & -0.079800974 \\
\hline 12.1 & 0 & 0.00114278 & -0.096540942 & -0.080941942 \\
\hline 12.2 & 0 & 0.000990866 & -0.097686855 & -0.082078604 \\
\hline 12.3 & 0 & 0.000840167 & -0.098828492 & -0.083210988 \\
\hline 12.4 & 0 & 0.000690677 & -0.099965882 & -0.084339126 \\
\hline 12.5 & 0 & 0.000542391 & -0.101099053 & -0.085463046 \\
\hline 12.6 & 0 & 0.0003953 & -0.102228035 & -0.086582776 \\
\hline 12.7 & 0 & 0.000249401 & -0.103352857 & -0.087698347 \\
\hline 12.8 & 0 & 0.000104687 & -0.104473547 & -0.088809785 \\
\hline 12.9 & 0 & -0.0000388485 & -0.105590132 & -0.089917119 \\
\hline 13 & 0 & -0.000181211 & -0.106702641 & -0.091020377 \\
\hline 13.1 & 0 & -0.000322405 & -0.1078111 & -0.092119585 \\
\hline 13.2 & 0 & -0.000462439 & -0.108915538 & -0.093214771 \\
\hline 13.3 & 0 & -0.000601316 & -0.110015979 & -0.094305962 \\
\hline 13.4 & 0 & -0.000739043 & -0.111112452 & -0.095393184 \\
\hline 13.5 & 0 & -0.000875626 & -0.112204981 & -0.096476464 \\
\hline 13.6 & 0 & -0.001011071 & -0.113293594 & -0.097555826 \\
\hline 13.7 & 0 & -0.001145381 & -0.114378315 & -0.098631297 \\
\hline 13.8 & 0 & -0.001278565 & -0.115459169 & -0.099702901 \\
\hline 13.9 & 0 & -0.001410626 & -0.116536182 & -0.100770665 \\
\hline
\end{tabular}




\begin{tabular}{|c|c|c|c|c|}
\hline 4 & 0 & -0.001541571 & -0.117609379 & -0.101834613 \\
\hline 14.1 & 0 & -0.001671404 & -0.118678784 & -0.102894769 \\
\hline 14.2 & 0 & -0.001800132 & -0.119744422 & -0.103951157 \\
\hline 14.3 & 0 & -0.00192776 & -0.120806315 & -0.105003802 \\
\hline 14.4 & 0 & -0.002054292 & -0.121864489 & -0.106052727 \\
\hline 14.5 & 0 & -0.002179735 & -0.122918966 & -0.107097956 \\
\hline 14.6 & 0 & -0.002304093 & -0.123969769 & -0.108139512 \\
\hline 14.7 & 0 & -0.002427373 & -0.125016923 & -0.109177417 \\
\hline 14.8 & 0 & -0.002549578 & -0.126060449 & -0.110211696 \\
\hline 14.9 & 0 & -0.002670714 & -0.127100369 & -0.11124237 \\
\hline 15 & 0 & -0.002790787 & -0.128136708 & -0.112269461 \\
\hline 15.1 & 0 & -0.002909801 & -0.129169485 & -0.113292992 \\
\hline 15.2 & 0 & -0.003027762 & -0.130198724 & -0.114312985 \\
\hline 15.3 & 0 & -0.003144674 & -0.131224445 & -0.115329461 \\
\hline 15.4 & 0 & -0.003260543 & -0.132246671 & -0.116342441 \\
\hline 15.5 & 0 & -0.003375373 & -0.133265422 & -0.117351947 \\
\hline 15.6 & 0 & -0.003489169 & -0.13428072 & -0.118358 \\
\hline 15.7 & 0 & -0.003601937 & -0.135292585 & -0.119360621 \\
\hline 15.8 & 0 & -0.003713681 & -0.136301038 & -0.120359829 \\
\hline 15.9 & 0 & -0.003824405 & -0.137306099 & -0.121355646 \\
\hline 16 & 0 & -0.003934116 & -0.138307788 & -0.122348092 \\
\hline 16.1 & 0 & -0.004042817 & -0.139306125 & -0.123337187 \\
\hline 16.2 & 0 & -0.004150513 & -0.140301131 & -0.12432295 \\
\hline 16.3 & 0 & -0.004257209 & -0.141292825 & -0.125305401 \\
\hline 16.4 & 0 & -0.00436291 & -0.142281225 & -0.12628456 \\
\hline 16.5 & 0 & -0.00446762 & -0.143266352 & -0.127260446 \\
\hline 16.6 & 0 & -0.004571344 & -0.144248224 & -0.128233077 \\
\hline 16.7 & 0 & -0.004674086 & -0.145226861 & -0.129202473 \\
\hline 16.8 & 0 & -0.004775851 & -0.14620228 & -0.130168652 \\
\hline 16.9 & 0 & -0.004876643 & -0.147174501 & -0.131131634 \\
\hline 17 & 0 & -0.004976467 & -0.148143542 & -0.132091435 \\
\hline 17.1 & 0 & -0.005075328 & -0.14910942 & -0.133048075 \\
\hline
\end{tabular}




$\begin{array}{lllll}17.2 & 0 & -0.005173229 & -0.150072154 & -0.134001571 \\ 17.3 & 0 & -0.005270176 & -0.151031763 & -0.134951941 \\ 17.4 & 0 & -0.005366171 & -0.151988262 & -0.135899203 \\ 17.5 & 0 & -0.005461221 & -0.152941671 & -0.136843375 \\ 17.6 & 0 & -0.005555328 & -0.153892005 & -0.137784474 \\ 17.7 & 0 & -0.005648498 & -0.154839283 & -0.138722516 \\ 17.8 & 0 & -0.005740735 & -0.155783522 & -0.139657519 \\ 17.9 & 0 & -0.005832042 & -0.156724738 & -0.1405895 \\ 18 & 0 & -0.005922424 & -0.157662948 & -0.141518476 \\ 18.1 & 0 & -0.006011886 & -0.158598168 & -0.142444463 \\ 18.2 & 0 & -0.00610043 & -0.159530416 & -0.143367477 \\ 18.3 & 0 & -0.006188062 & -0.160459707 & -0.144287535 \\ 18.4 & 0 & -0.006274785 & -0.161386057 & -0.145204654 \\ 18.5 & 0 & -0.006360603 & -0.162309483 & -0.146118848 \\ 18.6 & 0 & -0.006445521 & -0.16323 & -0.147030134 \\ 18.7 & 0 & -0.006529542 & -0.164147625 & -0.147938528 \\ 18.8 & 0 & -0.006612671 & -0.165062371 & -0.148844045 \\ 18.9 & 0 & -0.006694911 & -0.165974256 & -0.1497467 \\ 19 & 0 & -0.006776265 & -0.166883295 & -0.15064651 \\ 19.9 & 0 & -0.006856739 & -0.167789501 & -0.151543488 \\ 19.6 & 0 & -0.007246028 & -0.172278585 & -0.155986441 \\ 19.2 & 0 & -0.006936336 & -0.168692892 & -0.152437651 \\ 19.4 & 0 & -0.007092913 & -0.170491282 & -0.154217589 \\ 19.5 & 0 & -0.007169902 & -0.171386313 & -0.155103393 \\ 19.6 & -0.007321296 & -0.173168114 & -0.156866745 \\ 19.0 & 0.007395709 & -0.174054915 & -0.157744322 \\ 1907469272 & -0.174939001 & -0.158619185 \\ 19.007541987 & -0.175820387 & -0.159491348\end{array}$


The Gibbs free energy difference of the four polytypes of boron nitride ( $\mathrm{r}-\mathrm{BN}, \mathrm{h}-\mathrm{BN}, \mathrm{c}-\mathrm{BN}$, and w-BN) as a function of pressure ranging from 0 to $20 \mathrm{GPa}$, calculated with respect to $\mathrm{r}-\mathrm{BN}$ as a reference at a given temperature of $\mathbf{2 2 0 0}$ Kelvin. The unit of the Gibbs free energy difference for the four polytypes of boron nitride is given in $\mathrm{eV} /$ atom.

\begin{tabular}{lllll}
$\mathbf{P}(\mathbf{G P a}) \mathbf{r}-\mathbf{B N}$ & $\mathbf{h}-\mathbf{B N}$ & $\mathbf{c}-\mathbf{B N}$ & $\mathbf{w}-\mathbf{B N}$ \\
0 & 0 & 0.03140529 & 0.092476881 & 0.106813902 \\
0.1 & 0 & 0.031094504 & 0.090364584 & 0.104710726 \\
0.2 & 0 & 0.030781595 & 0.088267899 & 0.102623165 \\
0.3 & 0 & 0.030466931 & 0.086186541 & 0.100550932 \\
0.4 & 0 & 0.030150836 & 0.084120233 & 0.098493752 \\
0.5 & 0 & 0.029833603 & 0.082068708 & 0.096451356 \\
0.6 & 0 & 0.029515496 & 0.080031707 & 0.094423486 \\
0.7 & 0 & 0.029196748 & 0.078008982 & 0.092409893 \\
0.8 & 0 & 0.028877574 & 0.076000288 & 0.090410334 \\
0.9 & 0 & 0.028558166 & 0.074005393 & 0.088424574 \\
1 & 0 & 0.028238698 & 0.072024067 & 0.086452386 \\
1.1 & 0 & 0.027919329 & 0.070056092 & 0.08449355 \\
1.2 & 0 & 0.027600201 & 0.068101253 & 0.082547852 \\
1.3 & 0 & 0.027281446 & 0.066159341 & 0.080615083 \\
1.4 & 0 & 0.026963183 & 0.064230157 & 0.078695042 \\
1.5 & 0 & 0.026645519 & 0.062313503 & 0.076787534 \\
1.6 & 0 & 0.026328554 & 0.060409189 & 0.074892367 \\
1.7 & 0 & 0.026012378 & 0.05851703 & 0.073009357 \\
1.8 & 0 & 0.025697074 & 0.056636846 & 0.071138323 \\
1.9 & 0 & 0.025382717 & 0.054768461 & 0.06927909 \\
2 & 0 & 0.025069377 & 0.052911706 & 0.067431488 \\
2.1 & 0 & 0.024757115 & 0.051066413 & 0.06559535 \\
2.2 & 0 & 0.024445991 & 0.049232422 & 0.063770514 \\
2.3 & 0 & 0.024136056 & 0.047409573 & 0.061956823 \\
& 0 & 0.023827359 & 0.045597714 & 0.060154123 \\
\hline .6 & 0 & 0.023519945 & 0.043796695 & 0.058362264 \\
0.023213854 & 0.042006369 & 0.0565811
\end{tabular}




\begin{tabular}{|c|c|c|c|c|}
\hline .7 & 0 & 0.022909122 & 0.040226594 & 0.054810488 \\
\hline 2.8 & 0 & 0.022605784 & 0.03845723 & 0.053050289 \\
\hline 2.9 & 0 & 0.022303871 & 0.036698142 & 0.051300366 \\
\hline 3 & 0 & 0.022003409 & 0.034949196 & 0.049560587 \\
\hline 3.1 & 0 & 0.021704425 & 0.033210263 & 0.047830822 \\
\hline 3.2 & 0 & 0.021406942 & 0.031481216 & 0.046110945 \\
\hline 3.3 & 0 & 0.021110982 & 0.029761932 & 0.044400832 \\
\hline 3.4 & 0 & 0.020816562 & 0.028052289 & 0.042700361 \\
\hline 3.5 & 0 & 0.020523701 & 0.026352168 & 0.041009414 \\
\hline 3.6 & 0 & 0.020232413 & 0.024661455 & 0.039327875 \\
\hline 3.7 & 0 & 0.019942714 & 0.022980035 & 0.037655631 \\
\hline 3.8 & 0 & 0.019654614 & 0.021307798 & 0.03599257 \\
\hline 3.9 & 0 & 0.019368126 & 0.019644635 & 0.034338585 \\
\hline 4 & 0 & 0.019083259 & 0.01799044 & 0.032693569 \\
\hline 4.1 & 0 & 0.018800021 & 0.016345108 & 0.031057419 \\
\hline 4.2 & 0 & 0.018518421 & 0.014708539 & 0.029430031 \\
\hline 4.3 & 0 & 0.018238464 & 0.013080631 & 0.027811306 \\
\hline 4.4 & 0 & 0.017960157 & 0.011461288 & 0.026201146 \\
\hline 4.5 & 0 & 0.017683504 & 0.009850413 & 0.024599456 \\
\hline 4.6 & 0 & 0.017408509 & 0.008247912 & 0.023006141 \\
\hline 4.7 & 0 & 0.017135174 & 0.006653694 & 0.021421109 \\
\hline 4.8 & 0 & 0.016863503 & 0.005067667 & 0.01984427 \\
\hline 4.9 & 0 & 0.016593496 & 0.003489743 & 0.018275535 \\
\hline 5 & 0 & 0.016325156 & 0.001919835 & 0.016714817 \\
\hline 5.1 & 0 & 0.016058482 & 0.000357858 & 0.015162031 \\
\hline 5.2 & 0 & 0.015793475 & -0.001196271 & 0.013617093 \\
\hline 5.3 & 0 & 0.015530133 & -0.002742636 & 0.012079921 \\
\hline 5.4 & 0 & 0.015268457 & -0.004281317 & 0.010550434 \\
\hline 5.5 & 0 & 0.015008444 & -0.005812393 & 0.009028553 \\
\hline 5.6 & 0 & 0.014750092 & -0.007335941 & 0.0075142 \\
\hline 5.7 & 0 & 0.0144934 & -0.008852039 & 0.006007299 \\
\hline 5.8 & 0 & 0.014238364 & -0.010360761 & 0.004507774 \\
\hline
\end{tabular}




\begin{tabular}{|c|c|c|c|c|}
\hline 5.9 & 0 & 0.013984982 & -0.011862181 & 0.003015553 \\
\hline 6 & 0 & 0.013733251 & -0.013356371 & 0.001530562 \\
\hline 6.1 & 0 & 0.013483167 & -0.014843402 & 0.0000527298 \\
\hline 6.2 & 0 & 0.013234725 & -0.016323346 & -0.001418013 \\
\hline 6.3 & 0 & 0.012987923 & -0.017796269 & -0.002881735 \\
\hline 6.4 & 0 & 0.012742756 & -0.019262241 & -0.004338504 \\
\hline 6.5 & 0 & 0.012499219 & -0.020721327 & -0.005788387 \\
\hline 6.6 & 0 & 0.012257308 & -0.022173592 & -0.007231448 \\
\hline 6.7 & 0 & 0.012017017 & -0.023619102 & -0.008667754 \\
\hline 6.8 & 0 & 0.011778342 & -0.02505792 & -0.010097366 \\
\hline 6.9 & 0 & 0.011541278 & -0.026490106 & -0.011520346 \\
\hline 7 & 0 & 0.011305819 & -0.027915724 & -0.012936757 \\
\hline 7.1 & 0 & 0.011071959 & -0.029334833 & -0.014346659 \\
\hline 7.2 & 0 & 0.010839694 & -0.030747493 & -0.01575011 \\
\hline 7.3 & 0 & 0.010609017 & -0.032153761 & -0.01714717 \\
\hline 7.4 & 0 & 0.010379922 & -0.033553696 & -0.018537895 \\
\hline 7.5 & 0 & 0.010152403 & -0.034947353 & -0.019922342 \\
\hline 7.6 & 0 & 0.009926456 & -0.03633479 & -0.021300568 \\
\hline 7.7 & 0 & 0.009702072 & -0.03771606 & -0.022672626 \\
\hline 7.8 & 0 & 0.009479247 & -0.039091217 & -0.024038572 \\
\hline 7.9 & 0 & 0.009257973 & -0.040460315 & -0.025398457 \\
\hline 8 & 0 & 0.009038245 & -0.041823407 & -0.026752335 \\
\hline 8.1 & 0 & 0.008820057 & -0.043180543 & -0.028100258 \\
\hline 8.2 & 0 & 0.008603401 & -0.044531775 & -0.029442275 \\
\hline 8.3 & 0 & 0.008388271 & -0.045877153 & -0.030778439 \\
\hline 8.4 & 0 & 0.008174662 & -0.047216727 & -0.032108797 \\
\hline 8.5 & 0 & 0.007962565 & -0.048550544 & -0.033433398 \\
\hline 8.6 & 0 & 0.007751975 & -0.049878654 & -0.034752291 \\
\hline 8.7 & 0 & 0.007542885 & -0.051201103 & -0.036065523 \\
\hline 8.8 & 0 & 0.007335289 & -0.052517938 & -0.03737314 \\
\hline 8.9 & 0 & 0.00712918 & -0.053829205 & -0.03867519 \\
\hline 9 & 0 & 0.00692455 & -0.055134949 & -0.039971716 \\
\hline
\end{tabular}




\begin{tabular}{|c|c|c|c|c|}
\hline .1 & 0 & 0.006721395 & -0.056435216 & -0.041262764 \\
\hline 9.2 & 0 & 0.006519706 & -0.057730049 & -0.042548378 \\
\hline 9.3 & 0 & 0.006319477 & -0.059019492 & -0.043828601 \\
\hline 9.4 & & 0.006120701 & -0.060303588 & -0.045103476 \\
\hline 9.5 & 0 & 0.005923373 & -0.061582379 & -0.046373047 \\
\hline 9.6 & 0 & 0.005727484 & -0.062855906 & -0.047637354 \\
\hline 9.7 & 0 & 0.005533029 & -0.064124212 & -0.048896438 \\
\hline 9.8 & 0 & 0.005340001 & -0.065387337 & -0.050150341 \\
\hline 9.9 & 0 & 0.005148392 & -0.06664532 & -0.051399102 \\
\hline 10 & 0 & 0.004958198 & -0.067898203 & -0.052642762 \\
\hline 10.1 & 0 & 0.004769409 & -0.069146023 & -0.05388136 \\
\hline 10.2 & 0 & 0.004582021 & -0.070388819 & -0.055114933 \\
\hline 10.3 & 0 & 0.004396027 & -0.071626629 & -0.05634352 \\
\hline 10.4 & 0 & 0.004211419 & -0.072859491 & -0.057567158 \\
\hline 10.5 & 0 & 0.004028192 & -0.074087443 & -0.058785886 \\
\hline 10.6 & 0 & 0.003846339 & -0.07531052 & -0.059999739 \\
\hline 10.7 & 0 & 0.003665853 & -0.07652876 & -0.061208754 \\
\hline 10.8 & 0 & 0.003486727 & -0.077742197 & -0.062412966 \\
\hline 10.9 & 0 & 0.003308956 & -0.078950867 & -0.063612412 \\
\hline 11 & 0 & 0.003132533 & -0.080154805 & -0.064807124 \\
\hline 11.1 & 0 & 0.002957451 & -0.081354045 & -0.065997139 \\
\hline 11.2 & 0 & 0.002783704 & -0.082548622 & -0.067182491 \\
\hline 11.3 & 0 & 0.002611285 & -0.083738569 & -0.068363212 \\
\hline 11.4 & 0 & 0.002440189 & -0.084923919 & -0.069539336 \\
\hline 11.5 & 0 & 0.002270408 & -0.086104705 & -0.070710896 \\
\hline 11.6 & 0 & 0.002101937 & -0.08728096 & -0.071877924 \\
\hline 11.7 & 0 & 0.001934769 & -0.088452715 & -0.073040453 \\
\hline 11.8 & 0 & 0.001768898 & -0.089620002 & -0.074198513 \\
\hline 11.9 & 0 & 0.001604318 & -0.090782853 & -0.075352137 \\
\hline 12 & 0 & 0.001441023 & -0.091941298 & -0.076501355 \\
\hline 12.1 & 0 & 0.001279006 & -0.093095368 & -0.077646198 \\
\hline 12.2 & 0 & 0.001118261 & -0.094245093 & -0.078786696 \\
\hline
\end{tabular}




\begin{tabular}{|c|c|c|c|c|}
\hline 12.3 & 0 & 0.000958782 & -0.095390503 & -0.079922878 \\
\hline 12.4 & 0 & 0.000800564 & -0.096531627 & -0.081054776 \\
\hline 12.5 & 0 & 0.000643599 & -0.097668495 & -0.082182416 \\
\hline 12.6 & 0 & 0.000487883 & -0.098801136 & -0.083305829 \\
\hline 12.7 & 0 & 0.000333408 & -0.099929577 & -0.084425044 \\
\hline 2.8 & 0 & 0.00018017 & -0.101053848 & -0.085540087 \\
\hline 12.9 & 0 & 0.0000281626 & -0.102173976 & -0.086650987 \\
\hline 13 & 0 & -0.000122621 & -0.103289989 & -0.087757773 \\
\hline 13.1 & 0 & -0.000272186 & -0.104401914 & -0.08886047 \\
\hline 13.2 & 0 & -0.000420538 & -0.105509778 & -0.089959106 \\
\hline 13.3 & 0 & -0.000567683 & -0.106613607 & -0.091053708 \\
\hline 13.4 & 0 & -0.000713626 & -0.107713429 & -0.092144302 \\
\hline 13.5 & 0 & -0.000858375 & -0.108809268 & -0.093230914 \\
\hline 13.6 & P & -0.001001933 & -0.109901152 & -0.09431357 \\
\hline 13.7 & 0 & -0.001144307 & -0.110989105 & -0.095392296 \\
\hline 13.8 & 0 & -0.001285502 & -0.112073153 & -0.096467116 \\
\hline 13.9 & 0 & -0.001425525 & -0.113153321 & -0.097538057 \\
\hline 14 & 0 & -0.001564379 & -0.114229634 & -0.098605142 \\
\hline 14.1 & 0 & -0.001702072 & -0.115302115 & -0.099668397 \\
\hline 14.2 & 0 & -0.001838607 & -0.11637079 & -0.100727844 \\
\hline 14.3 & 0 & -0.001973992 & -0.117435682 & -0.101783509 \\
\hline 14.4 & 0 & -0.002108231 & -0.118496816 & -0.102835416 \\
\hline 14.5 & 0 & -0.002241329 & -0.119554213 & -0.103883586 \\
\hline 14.6 & 0 & -0.002373293 & -0.120607898 & -0.104928045 \\
\hline 14.7 & 0 & -0.002504126 & -0.121657895 & -0.105968815 \\
\hline 14.8 & 0 & -0.002633835 & -0.122704224 & -0.107005918 \\
\hline 14.9 & 0 & -0.002762425 & -0.12374691 & -0.108039377 \\
\hline 15 & 0 & -0.0028899 & -0.124785974 & -0.109069215 \\
\hline 15.1 & 0 & -0.003016267 & -0.125821438 & -0.110095454 \\
\hline 15.2 & 0 & -0.00314153 & -0.126853325 & -0.111118115 \\
\hline 15.3 & 0 & -0.003265694 & -0.127881656 & -0.11213722 \\
\hline 15.4 & 0 & -0.003388764 & -0.128906452 & -0.113152791 \\
\hline
\end{tabular}




\begin{tabular}{|c|c|c|c|c|}
\hline 15.5 & 0 & -0.003510745 & -0.129927735 & -0.114164848 \\
\hline 15.6 & 0 & -0.003631643 & -0.130945525 & -0.115173414 \\
\hline 15.7 & 0 & -0.003751463 & -0.131959844 & -0.116178508 \\
\hline 15.8 & 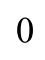 & -0.003870208 & -0.132970712 & -0.117180151 \\
\hline 15.9 & 0 & -0.003987885 & -0.133978149 & -0.118178365 \\
\hline 16 & 0 & -0.004104497 & -0.134982176 & -0.119173168 \\
\hline 16.1 & 0 & -0.004220051 & -0.135982813 & -0.120164581 \\
\hline 16.2 & 0 & -0.00433455 & -0.136980079 & -0.121152624 \\
\hline 16.3 & 0 & -0.004447999 & -0.137973994 & -0.122137316 \\
\hline 16.4 & 0 & -0.004560404 & -0.138964578 & -0.123118677 \\
\hline 16.5 & 0 & -0.004671769 & -0.13995185 & -0.124096726 \\
\hline 16.6 & 0 & -0.004782098 & -0.140935829 & -0.125071483 \\
\hline 16.7 & 0 & -0.004891397 & -0.141916534 & -0.126042965 \\
\hline 16.8 & 0 & -0.004999669 & -0.142893983 & -0.127011193 \\
\hline 16.9 & 0 & -0.00510692 & -0.143868195 & -0.127976184 \\
\hline 17 & 0 & -0.005213154 & -0.144839189 & -0.128937957 \\
\hline 17.1 & 0 & -0.005318375 & -0.145806983 & -0.12989653 \\
\hline 17.2 & 0 & -0.005422589 & -0.146771594 & -0.130851921 \\
\hline 17.3 & 0 & -0.005525799 & -0.147733041 & -0.131804149 \\
\hline 17.4 & 0 & -0.00562801 & -0.148691342 & -0.132753229 \\
\hline 17.5 & 0 & -0.005729226 & -0.149646513 & -0.133699182 \\
\hline 17.6 & 0 & -0.005829452 & -0.150598573 & -0.134642023 \\
\hline 17.7 & 0 & -0.005928692 & -0.151547538 & -0.13558177 \\
\hline 17.8 & 0 & -0.006026951 & -0.152493426 & -0.13651844 \\
\hline 17.9 & 0 & -0.006124233 & -0.153436254 & -0.13745205 \\
\hline 18 & 0 & -0.006220541 & -0.154376038 & -0.138382616 \\
\hline 18.1 & 0 & -0.006315881 & -0.155312795 & -0.139310157 \\
\hline 18.2 & 0 & -0.006410257 & -0.156246542 & -0.140234687 \\
\hline 18.3 & 0 & -0.006503672 & -0.157177294 & -0.141156223 \\
\hline 18.4 & 0 & -0.00659613 & -0.158105068 & -0.142074782 \\
\hline 18.5 & 0 & -0.006687637 & -0.159029881 & -0.14299038 \\
\hline 18.6 & 0 & -0.006778196 & -0.159951747 & -0.143903032 \\
\hline
\end{tabular}




$\begin{array}{lllll}18.7 & 0 & -0.006867812 & -0.160870684 & -0.144812754 \\ 18.8 & 0 & -0.006956487 & -0.161786705 & -0.145719562 \\ 18.9 & 0 & -0.007044227 & -0.162699828 & -0.146623472 \\ 19 & 0 & -0.007131035 & -0.163610067 & -0.147524498 \\ 19.1 & 0 & -0.007216915 & -0.164517438 & -0.148422657 \\ 19.2 & 0 & -0.007301872 & -0.165421955 & -0.149317963 \\ 19.3 & 0 & -0.007385908 & -0.166323634 & -0.150210431 \\ 19.4 & 0 & -0.007469029 & -0.16722249 & -0.151100075 \\ 19.5 & 0 & -0.007551238 & -0.168118537 & -0.151986912 \\ 19.6 & 0 & -0.007632538 & -0.16901179 & -0.152870956 \\ 19.7 & 0 & -0.007712934 & -0.169902263 & -0.15375222 \\ 19.8 & 0 & -0.007792429 & -0.170789971 & -0.154630719 \\ 19.9 & 0 & -0.007871028 & -0.171674928 & -0.155506468 \\ 20 & 0 & -0.007948734 & -0.172557148 & -0.156379481\end{array}$

The Gibbs free energy difference of the four polytypes of boron nitride ( $\mathrm{r}-\mathrm{BN}, \mathrm{h}-\mathrm{BN}, \mathrm{c}-\mathrm{BN}$, and w-BN) as a function of pressure ranging from 0 to $20 \mathrm{GPa}$, calculated with respect to $\mathrm{r}-\mathrm{BN}$ as a reference at a given temperature of 2300 Kelvin. The unit of the Gibbs free energy difference for the four polytypes of boron nitride is given in $\mathrm{eV} /$ atom.

\begin{tabular}{lllll}
\multicolumn{2}{l}{$\mathbf{P}(\mathbf{G P a}) \mathbf{r}-\mathbf{B N}$} & $\mathbf{h}-\mathbf{B N}$ & $\mathbf{c}-\mathbf{B N}$ & $\mathbf{w}-\mathbf{B N}$ \\
0 & 0 & 0.032839881 & 0.096405841 & 0.110595609 \\
0.1 & 0 & 0.032522389 & 0.094294707 & 0.108493553 \\
0.2 & 0 & 0.032202464 & 0.09219882 & 0.106406746 \\
0.3 & 0 & 0.031880489 & 0.090117912 & 0.10433492 \\
0.4 & 0 & 0.031556806 & 0.088051725 & 0.102277817 \\
0.5 & 0 & 0.031231722 & 0.086000009 & 0.100235188 \\
0.6 & 0 & 0.030905513 & 0.083962521 & 0.098206788 \\
0.7 & 0 & 0.03057843 & 0.081939028 & 0.096192385 \\
0.8 & 0 & 0.030250698 & 0.079929299 & 0.094191749 \\
0.9 & 0 & 0.029922521 & 0.077933116 & 0.092204659 \\
1 & 0 & 0.029594086 & 0.075950263 & 0.090230902 \\
1.1 & 0 & 0.029265561 & 0.073980531 & 0.088270268
\end{tabular}




\begin{tabular}{|c|c|c|c|c|}
\hline 1.2 & 0 & 0.028937102 & 0.072023719 & 0.086322556 \\
\hline 1.3 & 0 & 0.028608847 & 0.07007963 & 0.084387568 \\
\hline 1.4 & 0 & 0.028280926 & 0.068148072 & 0.082465113 \\
\hline 1.5 & 0 & 0.027953455 & 0.06622886 & 0.080555006 \\
\hline 1.6 & 0 & 0.027626543 & 0.064321812 & 0.078657065 \\
\hline 1.7 & 0 & 0.027300288 & 0.062426752 & 0.076771114 \\
\hline 1.8 & 0 & 0.02697478 & 0.060543509 & 0.074896981 \\
\hline 1.9 & 0 & 0.026650103 & 0.058671915 & 0.073034499 \\
\hline 2 & 0 & 0.026326331 & 0.056811808 & 0.071183506 \\
\hline 2.1 & 0 & 0.026003535 & 0.054963028 & 0.069343842 \\
\hline 2.2 & 0 & 0.02568178 & 0.053125422 & 0.067515352 \\
\hline 2.3 & 0 & 0.025361123 & 0.051298837 & 0.065697887 \\
\hline 2.4 & 0 & 0.02504162 & 0.049483127 & 0.063891297 \\
\hline 2.5 & 0 & 0.02472332 & 0.047678149 & 0.06209544 \\
\hline 2.6 & 0 & 0.024406269 & 0.04588376 & 0.060310175 \\
\hline 2.7 & 0 & 0.024090508 & 0.044099825 & 0.058535365 \\
\hline 2.8 & 0 & 0.023776077 & 0.042326209 & 0.056770876 \\
\hline 2.9 & 0 & 0.023463012 & 0.040562782 & 0.055016578 \\
\hline 3 & 0 & 0.023151343 & 0.038809415 & 0.053272341 \\
\hline 3.1 & 0 & 0.022841102 & 0.037065984 & 0.051538042 \\
\hline 3.2 & 0 & 0.022532315 & 0.035332367 & 0.049813557 \\
\hline 3.3 & 0 & 0.022225008 & 0.033608443 & 0.048098768 \\
\hline 3.4 & 0 & 0.021919203 & 0.031894097 & 0.046393557 \\
\hline 3.5 & 0 & 0.021614921 & 0.030189212 & 0.04469781 \\
\hline 3.6 & 0 & 0.021312181 & 0.028493678 & 0.043011414 \\
\hline 3.7 & 0 & 0.021011001 & 0.026807384 & 0.041334261 \\
\hline 3.8 & 0 & 0.020711395 & 0.025130224 & 0.039666242 \\
\hline 3.9 & 0 & 0.020413379 & 0.023462092 & 0.038007252 \\
\hline 4 & 0 & 0.020116964 & 0.021802885 & 0.036357189 \\
\hline 4.1 & 0 & 0.019822162 & 0.020152501 & 0.034715952 \\
\hline 4.2 & 0 & 0.019528984 & 0.018510843 & 0.03308344 \\
\hline 4.3 & 0 & 0.019237438 & 0.016877812 & 0.031459558 \\
\hline
\end{tabular}




\begin{tabular}{|c|c|c|c|c|}
\hline & 0 & 0.018947533 & 0.015253314 & 0.029844209 \\
\hline 4.5 & 0 & 0.018659275 & 0.013637256 & 0.028237302 \\
\hline 4.6 & 0 & 0.018372671 & 0.012029546 & 0.026638744 \\
\hline 47 & & 0.018087726 & 0.010430095 & 0.025048446 \\
\hline 4.8 & 0 & 0.017804445 & 0.008838813 & 0.023466319 \\
\hline 4.9 & 0 & 0.01752283 & 0.007255616 & 0.021892277 \\
\hline 5 & 0 & 0.017242886 & 0.005680418 & 0.020326236 \\
\hline 5.1 & 0 & 0.016964614 & 0.004113135 & 0.018768112 \\
\hline 5.2 & 0 & 0.016688016 & 0.002553687 & 0.017217823 \\
\hline 5.3 & 0 & 0.016413094 & 0.001001993 & 0.015675289 \\
\hline 5.4 & 0 & 0.016139847 & -0.000542027 & 0.014140431 \\
\hline 5.5 & 0 & 0.015868277 & -0.002078448 & 0.012613172 \\
\hline 5.6 & 0 & 0.015598383 & -0.003607348 & 0.011093436 \\
\hline 5.7 & 0 & 0.015330164 & -0.005128801 & 0.009581148 \\
\hline 5.8 & 0 & 0.015063619 & -0.006642881 & 0.008076234 \\
\hline 5.9 & 0 & 0.014798746 & -0.008149659 & 0.006578623 \\
\hline 6 & 0 & 0.014535543 & -0.009649208 & 0.005088242 \\
\hline 6.1 & 0 & 0.014274009 & -0.011141595 & 0.003605024 \\
\hline 6.2 & 0 & 0.01401414 & -0.012626891 & 0.002128898 \\
\hline 6.3 & 0 & 0.013755933 & -0.014105163 & 0.000659798 \\
\hline 6.4 & 0 & 0.013499386 & -0.015576476 & -0.000802343 \\
\hline 6.5 & 0 & 0.013244495 & -0.017040896 & -0.00225759 \\
\hline 6.6 & 0 & 0.012991257 & -0.018498488 & -0.003706008 \\
\hline 6.7 & 0 & 0.012739667 & -0.019949314 & -0.005147659 \\
\hline 6.8 & 0 & 0.012489721 & -0.021393437 & -0.006582607 \\
\hline 6.9 & 0 & 0.012241416 & -0.022830918 & -0.00801091 \\
\hline 7 & 0 & 0.011994746 & -0.024261817 & -0.009432632 \\
\hline 7.1 & 0 & 0.011749707 & -0.025686193 & -0.010847829 \\
\hline 7.2 & 0 & 0.011506294 & -0.027104106 & -0.012256562 \\
\hline 7.3 & 0 & 0.011264503 & -0.028515611 & -0.013658887 \\
\hline 7.4 & 0 & 0.011024328 & -0.029920766 & -0.015054861 \\
\hline 7.5 & 0 & 0.010785764 & -0.031319627 & -0.01644454 \\
\hline
\end{tabular}




\begin{tabular}{|c|c|c|c|c|}
\hline 7.6 & 0 & 0.010548805 & -0.032712248 & -0.017827978 \\
\hline 7.7 & 0 & 0.010313447 & -0.034098683 & -0.01920523 \\
\hline 7.8 & 0 & 0.010079683 & -0.035478987 & -0.020576348 \\
\hline 7.9 & 0 & 0.009847509 & -0.03685321 & -0.021941387 \\
\hline 8 & 0 & 0.009616918 & -0.038221406 & -0.023300396 \\
\hline 8.1 & 0 & 0.009387904 & -0.039583624 & -0.024653428 \\
\hline 8.2 & 0 & 0.009160462 & -0.040939916 & -0.026000532 \\
\hline 8.3 & 0 & 0.008934586 & -0.04229033 & -0.027341758 \\
\hline 8.4 & 0 & 0.008710269 & -0.043634916 & -0.028677155 \\
\hline 8.5 & 0 & 0.008487506 & -0.044973721 & -0.030006771 \\
\hline 8.6 & 0 & 0.00826629 & -0.046306794 & -0.031330653 \\
\hline 8.7 & 0 & 0.008046616 & -0.04763418 & -0.032648848 \\
\hline 8.8 & 0 & 0.007828478 & -0.048955926 & -0.033961403 \\
\hline 8.9 & 0 & 0.007611868 & -0.050272078 & -0.035268362 \\
\hline 9 & 0 & 0.007396781 & -0.05158268 & -0.036569771 \\
\hline 9.1 & 0 & 0.007183211 & -0.052887777 & -0.037865674 \\
\hline 9.2 & 0 & 0.006971151 & -0.054187411 & -0.039156115 \\
\hline 9.3 & 0 & 0.006760594 & -0.055481627 & -0.040441136 \\
\hline 9.4 & 0 & 0.006551535 & -0.056770467 & -0.04172078 \\
\hline 9.5 & 0 & 0.006343968 & -0.058053973 & -0.04299509 \\
\hline 9.6 & 0 & 0.006137885 & -0.059332186 & -0.044264106 \\
\hline 9.7 & 0 & 0.00593328 & -0.060605146 & -0.04552787 \\
\hline 9.8 & 0 & 0.005730148 & -0.061872895 & -0.046786422 \\
\hline 9.9 & 0 & 0.005528481 & -0.063135472 & -0.0480398 \\
\hline 10 & 0 & 0.005328273 & -0.064392917 & -0.049288046 \\
\hline 10.1 & 0 & 0.005129518 & -0.065645267 & -0.050531198 \\
\hline 10.2 & 0 & 0.00493221 & -0.066892562 & -0.051769293 \\
\hline 10.3 & 0 & 0.004736341 & -0.068134839 & -0.05300237 \\
\hline 10.4 & 0 & 0.004541907 & -0.069372136 & -0.054230466 \\
\hline 10.5 & 0 & 0.004348899 & -0.070604489 & -0.055453618 \\
\hline 10.6 & 0 & 0.004157313 & -0.071831935 & -0.056671862 \\
\hline 10.7 & 0 & 0.003967141 & -0.073054509 & -0.057885235 \\
\hline
\end{tabular}




\begin{tabular}{|c|c|c|c|c|}
\hline 10.8 & 0 & 0.003778377 & -0.074272248 & -0.059093771 \\
\hline 10.9 & 0 & 0.003591015 & -0.075485186 & -0.060297507 \\
\hline 11 & 0 & 0.003405049 & -0.076693358 & -0.061496475 \\
\hline 11.1 & 0 & 0.003220472 & -0.077896798 & -0.062690712 \\
\hline 11.2 & 0 & 0.003037278 & -0.07909554 & -0.06388025 \\
\hline 1.3 & 0 & 0.00285546 & -0.080289617 & -0.065065124 \\
\hline 11.4 & 0 & 0.002675014 & -0.081479063 & -0.066245365 \\
\hline 11.5 & 0 & 0.002495931 & -0.08266391 & -0.067421007 \\
\hline 11.6 & 0 & 0.002318207 & -0.08384419 & -0.068592082 \\
\hline 11.7 & 0 & 0.002141835 & -0.085019936 & -0.069758621 \\
\hline 11.8 & 0 & 0.001966808 & -0.086191177 & -0.070920658 \\
\hline 11.9 & 0 & 0.001793121 & -0.087357947 & -0.072078221 \\
\hline 12 & 0 & 0.001620768 & -0.088520275 & -0.073231343 \\
\hline 12.1 & 0 & 0.001449742 & -0.089678192 & -0.074380053 \\
\hline 12.2 & 0 & 0.001280037 & -0.090831728 & -0.075524382 \\
\hline 12.3 & 0 & 0.001111648 & -0.091980913 & -0.07666436 \\
\hline 12.4 & 0 & 0.000944568 & -0.093125776 & -0.077800015 \\
\hline 12.5 & 0 & 0.000778791 & -0.094266346 & -0.078931378 \\
\hline 12.6 & 0 & 0.000614312 & -0.095402652 & -0.080058476 \\
\hline 12.7 & 0 & 0.000451125 & -0.096534723 & -0.081181338 \\
\hline 12.8 & 0 & 0.000289223 & -0.097662586 & -0.082299993 \\
\hline 12.9 & 0 & 0.000128601 & -0.098786269 & -0.083414468 \\
\hline 13 & 0 & -0.0000307474 & -0.0999058 & -0.08452479 \\
\hline 13.1 & 0 & -0.000188828 & -0.101021206 & -0.085630987 \\
\hline 13.2 & 0 & -0.000345645 & -0.102132513 & -0.086733086 \\
\hline 13.3 & 0 & -0.000501207 & -0.10323975 & -0.087831113 \\
\hline 13.4 & 0 & -0.000655517 & -0.10434294 & -0.088925095 \\
\hline 13.5 & 0 & -0.000808582 & -0.105442112 & -0.090015057 \\
\hline 13.6 & 0 & -0.000960408 & -0.106537291 & -0.091101026 \\
\hline 13.7 & 0 & -0.001110999 & -0.107628501 & -0.092183027 \\
\hline 13.8 & 0 & -0.001260363 & -0.108715769 & -0.093261085 \\
\hline 13.9 & 0 & -0.001408504 & -0.109799119 & -0.094335226 \\
\hline
\end{tabular}




\begin{tabular}{|c|c|c|c|c|}
\hline 4 & 0 & -0.001555427 & -0.110878576 & -0.095405473 \\
\hline 14.1 & 0 & -0.001701139 & -0.111954165 & -0.096471851 \\
\hline 14.2 & 0 & -0.001845645 & -0.113025909 & -0.097534385 \\
\hline 14.3 & 0 & -0.00198895 & -0.114093832 & -0.098593099 \\
\hline 14.4 & 0 & -0.002131059 & -0.115157959 & -0.099648016 \\
\hline 4.5 & 0 & -0.002271979 & -0.116218313 & -0.10069916 \\
\hline 14.6 & 0 & -0.002411714 & -0.117274917 & -0.101746553 \\
\hline 14.7 & 0 & -0.00255027 & -0.118327793 & -0.10279022 \\
\hline 14.8 & 0 & -0.002687652 & -0.119376965 & -0.103830182 \\
\hline 14.9 & 0 & -0.002823865 & -0.120422456 & -0.104866462 \\
\hline 15 & 0 & -0.002958914 & -0.121464287 & -0.105899083 \\
\hline 15.1 & 0 & -0.003092805 & -0.12250248 & -0.106928066 \\
\hline 15.2 & 0 & -0.003225544 & -0.123537058 & -0.107953434 \\
\hline 15.3 & 0 & -0.003357134 & -0.124568042 & -0.108975208 \\
\hline 15.4 & 0 & -0.003487581 & -0.125595454 & -0.10999341 \\
\hline 15.5 & 0 & -0.003616891 & -0.126619314 & -0.111008061 \\
\hline 15.6 & 0 & -0.003745067 & -0.127639644 & -0.112019181 \\
\hline 15.7 & 0 & -0.003872116 & -0.128656465 & -0.113026792 \\
\hline 15.8 & 0 & -0.003998042 & -0.129669796 & -0.114030914 \\
\hline 15.9 & 0 & -0.004122851 & -0.13067966 & -0.115031568 \\
\hline 16 & 0 & -0.004246546 & -0.131686075 & -0.116028774 \\
\hline 16.1 & 0 & -0.004369134 & -0.132689062 & -0.117022552 \\
\hline 16.2 & 0 & -0.004490618 & -0.133688641 & -0.118012922 \\
\hline 16.3 & 0 & -0.004611005 & -0.134684832 & -0.118999903 \\
\hline 16.4 & 0 & -0.004730297 & -0.135677653 & -0.119983516 \\
\hline 16.5 & 0 & -0.004848501 & -0.136667124 & -0.120963778 \\
\hline 16.6 & 0 & -0.004965622 & -0.137653264 & -0.12194071 \\
\hline 16.7 & 0 & -0.005081663 & -0.138636093 & -0.122914331 \\
\hline 16.8 & 0 & -0.005196629 & -0.139615629 & -0.123884658 \\
\hline 16.9 & 0 & -0.005310526 & -0.14059189 & -0.124851711 \\
\hline 17 & 0 & -0.005423357 & -0.141564895 & -0.125815509 \\
\hline 17.1 & 0 & -0.005535128 & -0.142534662 & -0.126776068 \\
\hline
\end{tabular}




\begin{tabular}{|c|c|c|c|c|}
\hline 17.2 & 0 & -0.005645842 & -0.143501209 & -0.127733408 \\
\hline 17.3 & 0 & -0.005755506 & -0.144464555 & -0.128687547 \\
\hline 17.4 & 0 & -0.005864122 & -0.145424716 & -0.129638502 \\
\hline 17.5 & 0 & -0.005971696 & -0.146381711 & -0.13058629 \\
\hline 17.6 & 0 & -0.006078231 & -0.147335557 & -0.13153093 \\
\hline 17.7 & 0 & -0.006183734 & -0.148286272 & -0.132472438 \\
\hline 17.8 & 0 & -0.006288206 & -0.149233871 & -0.133410832 \\
\hline 17.9 & 0 & -0.006391655 & -0.150178373 & -0.134346128 \\
\hline 18 & 0 & -0.006494083 & -0.151119794 & -0.135278344 \\
\hline 18.1 & 0 & -0.006595494 & -0.152058151 & -0.136207496 \\
\hline 18.2 & 0 & -0.006695894 & -0.152993461 & -0.137133601 \\
\hline 18.3 & 0 & -0.006795287 & -0.153925739 & -0.138056675 \\
\hline 18.4 & 0 & -0.006893676 & -0.154855002 & -0.138976734 \\
\hline 18.5 & 0 & -0.006991066 & -0.155781266 & -0.139893795 \\
\hline 18.6 & 0 & -0.007087461 & -0.156704548 & -0.140807873 \\
\hline 18.7 & 0 & -0.007182865 & -0.157624862 & -0.141718984 \\
\hline 18.8 & 0 & -0.007277283 & -0.158542225 & -0.142627144 \\
\hline 18.9 & 0 & -0.007370719 & -0.159456652 & -0.143532369 \\
\hline 19 & 0 & -0.007463176 & -0.160368159 & -0.144434674 \\
\hline 19.1 & 0 & -0.007554659 & -0.161276761 & -0.145334074 \\
\hline 19.2 & 0 & -0.007645172 & -0.162182472 & -0.146230585 \\
\hline 19.3 & 0 & -0.007734718 & -0.163085309 & -0.147124221 \\
\hline 19.4 & 0 & -0.007823303 & -0.163985286 & -0.148014998 \\
\hline 19.5 & 0 & -0.007910929 & -0.164882418 & -0.148902929 \\
\hline 19.6 & 0 & -0.007997601 & -0.16577672 & -0.149788031 \\
\hline 19.7 & 0 & -0.008083322 & -0.166668205 & -0.150670317 \\
\hline 19.8 & 0 & -0.008168097 & -0.167556889 & -0.151549803 \\
\hline 19.9 & 0 & -0.008251929 & -0.168442786 & -0.152426501 \\
\hline & 0 & -0.008334823 & -0.16932591 & -0.153300427 \\
\hline
\end{tabular}


The Gibbs free energy difference of the four polytypes of boron nitride ( $\mathrm{r}-\mathrm{BN}, \mathrm{h}-\mathrm{BN}, \mathrm{c}-\mathrm{BN}$, and w-BN) as a function of pressure ranging from 0 to $20 \mathrm{GPa}$, calculated with respect to $\mathrm{r}-\mathrm{BN}$ as a reference at a given temperature of $\mathbf{2 4 0 0}$ Kelvin. The unit of the Gibbs free energy difference for the four polytypes of boron nitride is given in $\mathrm{eV} /$ atom.

\begin{tabular}{|c|c|c|c|c|}
\hline \multicolumn{2}{|c|}{ P(GPa) r-BN } & \multirow{2}{*}{$\begin{array}{l}\text { h-BN } \\
0.034275919\end{array}$} & \multirow{2}{*}{$\begin{array}{l}\text { c-BN } \\
0.100281001\end{array}$} & \multirow{2}{*}{$\begin{array}{l}\text { w-BN } \\
0.114329288\end{array}$} \\
\hline 0 & 0 & & & \\
\hline 0.1 & 0 & 0.033952006 & 0.098171496 & 0.112228839 \\
\hline 0.2 & 0 & 0.033625359 & 0.096076876 & 0.110143277 \\
\hline 0.3 & 0 & 0.033296376 & 0.093996892 & 0.108072353 \\
\hline 0.4 & 0 & 0.032965412 & 0.091931302 & 0.106015824 \\
\hline 0.5 & 0 & 0.03263279 & 0.089879871 & 0.103973458 \\
\hline 0.6 & 0 & 0.032298798 & 0.087842373 & 0.101945026 \\
\hline 0.7 & 0 & 0.031963699 & 0.085818588 & 0.099930308 \\
\hline 0.8 & 0 & 0.031627731 & 0.083808299 & 0.097929089 \\
\hline 0.9 & 0 & 0.031291109 & 0.081811301 & 0.095941163 \\
\hline 1 & 0 & 0.03095403 & 0.079827389 & 0.093966325 \\
\hline 1.1 & 0 & 0.030616673 & 0.077856368 & 0.092004379 \\
\hline 1.2 & 0 & 0.030279203 & 0.075898046 & 0.090055135 \\
\hline 1.3 & 0 & 0.029941769 & 0.073952238 & 0.088118406 \\
\hline 1.4 & 0 & 0.029604509 & 0.072018761 & 0.08619401 \\
\hline 1.5 & 0 & 0.029267549 & 0.07009744 & 0.084281771 \\
\hline 1.6 & 0 & 0.028931004 & 0.068188102 & 0.082381518 \\
\hline 1.7 & 0 & 0.02859498 & 0.06629058 & 0.080493082 \\
\hline 1.8 & 0 & 0.028259575 & 0.06440471 & 0.078616301 \\
\hline 1.9 & 0 & 0.02792488 & 0.062530334 & 0.076751015 \\
\hline 2 & 0 & 0.027590977 & 0.060667296 & 0.074897068 \\
\hline 2.1 & 0 & 0.027257943 & 0.058815445 & 0.07305431 \\
\hline 2.2 & 0 & 0.026925847 & 0.056974632 & 0.071222592 \\
\hline 2.3 & 0 & 0.026594755 & 0.055144713 & 0.06940177 \\
\hline 2.4 & 0 & 0.026264727 & 0.053325548 & 0.067591702 \\
\hline 2.5 & 0 & 0.025935817 & 0.051516998 & 0.065792252 \\
\hline 2.6 & 0 & 0.025608078 & 0.049718928 & 0.064003284 \\
\hline
\end{tabular}




\begin{tabular}{|c|c|c|c|c|}
\hline 2.7 & 0 & 0.025281555 & 0.047931208 & 0.062224667 \\
\hline 2.8 & 0 & 0.024956293 & 0.046153709 & 0.060456273 \\
\hline 2.9 & 0 & 0.024632332 & 0.044386305 & 0.058697974 \\
\hline 3 & 0 & 0.024309707 & 0.042628872 & 0.056949649 \\
\hline 3.1 & 0 & 0.023988454 & 0.040881292 & 0.055211178 \\
\hline 3.2 & 0 & 0.023668604 & 0.039143445 & 0.053482441 \\
\hline 3.3 & 0 & 0.023350186 & 0.037415216 & 0.051763325 \\
\hline 3.4 & 0 & 0.023033226 & 0.035696493 & 0.050053716 \\
\hline 3.5 & 0 & 0.022717748 & 0.033987166 & 0.048353503 \\
\hline 3.6 & 0 & 0.022403775 & 0.032287125 & 0.046662579 \\
\hline 3.7 & 0 & 0.022091328 & 0.030596265 & 0.044980837 \\
\hline 3.8 & 0 & 0.021780425 & 0.028914482 & 0.043308173 \\
\hline 3.9 & 0 & 0.021471083 & 0.027241675 & 0.041644486 \\
\hline 4 & 0 & 0.021163319 & 0.025577743 & 0.039989676 \\
\hline 4.1 & 0 & 0.020857146 & 0.023922588 & 0.038343645 \\
\hline 4.2 & 0 & 0.020552577 & 0.022276116 & 0.036706297 \\
\hline 4.3 & 0 & 0.020249625 & 0.02063823 & 0.035077537 \\
\hline 4.4 & 0 & 0.019948299 & 0.01900884 & 0.033457274 \\
\hline 4.5 & 0 & 0.019648608 & 0.017387855 & 0.031845417 \\
\hline 4.6 & 0 & 0.019350563 & 0.015775185 & 0.030241877 \\
\hline 4.7 & 0 & 0.019054169 & 0.014170744 & 0.028646567 \\
\hline 4.8 & 0 & 0.018759434 & 0.012574446 & 0.0270594 \\
\hline 4.9 & 0 & 0.018466364 & 0.010986206 & 0.025480294 \\
\hline 5 & 0 & 0.018174963 & 0.009405943 & 0.023909165 \\
\hline 5.1 & 0 & 0.017885235 & 0.007833575 & 0.022345933 \\
\hline 5.2 & 0 & 0.017597185 & 0.006269022 & 0.020790517 \\
\hline 5.3 & 0 & 0.017310815 & 0.004712206 & 0.01924284 \\
\hline 5.4 & 0 & 0.017026128 & 0.003163051 & 0.017702824 \\
\hline 5.5 & 0 & 0.016743125 & 0.001621481 & 0.016170394 \\
\hline 5.6 & 0 & 0.016461808 & 0.0000874212 & 0.014645476 \\
\hline 5.7 & 0 & 0.016182177 & -0.001439201 & 0.013127996 \\
\hline 5.8 & 0 & 0.015904233 & -0.002958458 & 0.011617883 \\
\hline
\end{tabular}




\begin{tabular}{|c|c|c|c|c|}
\hline .9 & 0 & 0.015627975 & -0.004470419 & 0.010115066 \\
\hline 6 & 0 & 0.015353403 & -0.005975154 & 0.008619476 \\
\hline 6.1 & 0 & 0.015080516 & -0.007472732 & 0.007131045 \\
\hline 6.2 & 0 & 0.014809314 & -0.00896322 & 0.005649705 \\
\hline 6.3 & 0 & 0.014539793 & -0.010446683 & 0.00417539 \\
\hline 6.4 & 0 & 0.014271952 & -0.011923187 & 0.002708036 \\
\hline 6.5 & 0 & 0.014005789 & -0.013392796 & 0.001247578 \\
\hline 6.6 & 0 & 0.013741301 & -0.014855572 & -0.000206047 \\
\hline 6.7 & 0 & 0.013478486 & -0.016311578 & -0.0016529 \\
\hline 6.8 & 0 & 0.01321734 & -0.017760874 & -0.003093042 \\
\hline 6.9 & 0 & 0.01295786 & -0.019203521 & -0.004526535 \\
\hline 7 & 0 & 0.012700043 & -0.020639577 & -0.005953436 \\
\hline 7.1 & 0 & 0.012443885 & -0.022069101 & -0.007373804 \\
\hline 7.2 & 0 & 0.012189381 & -0.023492151 & -0.008787697 \\
\hline 7.3 & 0 & 0.011936529 & -0.024908782 & -0.01019517 \\
\hline 7.4 & 0 & 0.011685323 & -0.026319051 & -0.01159628 \\
\hline 7.5 & 0 & 0.011435759 & -0.027723011 & -0.012991081 \\
\hline 7.6 & 0 & 0.011187833 & -0.029120718 & -0.014379628 \\
\hline 7.7 & 0 & 0.010941539 & -0.030512224 & -0.015761973 \\
\hline 7.8 & 0 & 0.010696874 & -0.031897582 & -0.017138169 \\
\hline 7.9 & 0 & 0.010453832 & -0.033276844 & -0.018508267 \\
\hline 8 & 0 & 0.010212408 & -0.034650059 & -0.01987232 \\
\hline 8.1 & 0 & 0.009972597 & -0.03601728 & -0.021230376 \\
\hline 8.2 & 0 & 0.009734393 & -0.037378554 & -0.022582485 \\
\hline 8.3 & 0 & 0.009497792 & -0.038733931 & -0.023928697 \\
\hline 8.4 & 0 & 0.009262788 & -0.04008346 & -0.025269059 \\
\hline 8.5 & 0 & 0.009029375 & -0.041427187 & -0.026603619 \\
\hline 8.6 & 0 & 0.008797548 & -0.042765159 & -0.027932423 \\
\hline 8.7 & 0 & 0.008567301 & -0.044097422 & -0.029255518 \\
\hline 8.8 & 0 & 0.008338628 & -0.045424023 & -0.030572949 \\
\hline 8.9 & 0 & 0.008111524 & -0.046745005 & -0.031884762 \\
\hline 9 & 0 & 0.007885984 & -0.048060414 & -0.033191 \\
\hline
\end{tabular}




\begin{tabular}{|c|c|c|c|c|}
\hline .1 & 0 & 0.007662 & -0.049370292 & -0.034491708 \\
\hline 9.2 & 0 & 0.007439567 & -0.050674684 & -0.035786928 \\
\hline 9.3 & 0 & 0.00721868 & -0.051973631 & -0.037076703 \\
\hline 9.4 & & 0.006999332 & -0.053267176 & -0.038361075 \\
\hline 9.5 & 0 & 0.006781517 & -0.05455536 & -0.039640086 \\
\hline 9.6 & 0 & 0.00656523 & -0.055838224 & -0.040913776 \\
\hline 9.7 & 0 & 0.006350464 & -0.057115808 & -0.042182186 \\
\hline 9.8 & 0 & 0.006137213 & -0.058388153 & -0.043445356 \\
\hline 9.9 & 0 & 0.005925471 & -0.059655298 & -0.044703325 \\
\hline 10 & 0 & 0.005715232 & -0.060917281 & -0.045956133 \\
\hline 10.1 & 0 & 0.005506491 & -0.062174141 & -0.047203817 \\
\hline 10.2 & 0 & 0.00529924 & -0.063425916 & -0.048446415 \\
\hline 10.3 & 0 & 0.005093473 & -0.064672644 & -0.049683965 \\
\hline 10.4 & 0 & 0.004889186 & -0.065914361 & -0.050916504 \\
\hline 10.5 & 0 & 0.00468637 & -0.067151104 & -0.052144068 \\
\hline 10.6 & 0 & 0.004485022 & -0.068382908 & -0.053366694 \\
\hline 10.7 & 0 & 0.004285133 & -0.06960981 & -0.054584417 \\
\hline 10.8 & 0 & 0.004086699 & -0.070831845 & -0.055797273 \\
\hline 10.9 & 0 & 0.003889712 & -0.072049048 & -0.057005295 \\
\hline 11 & 0 & 0.003694167 & -0.073261452 & -0.058208519 \\
\hline 11.1 & 0 & 0.003500059 & -0.074469092 & -0.059406979 \\
\hline 11.2 & 0 & 0.003307379 & -0.075672002 & -0.060600708 \\
\hline 11.3 & 0 & 0.003116123 & -0.076870215 & -0.061789739 \\
\hline 11.4 & 0 & 0.002926285 & -0.078063763 & -0.062974105 \\
\hline 11.5 & 0 & 0.002737859 & -0.079252678 & -0.064153838 \\
\hline 11.6 & 0 & 0.002550837 & -0.080436994 & -0.065328971 \\
\hline 11.7 & 0 & 0.002365215 & -0.081616741 & -0.066499536 \\
\hline 11.8 & 0 & 0.002180986 & -0.08279195 & -0.067665562 \\
\hline 11.9 & 0 & 0.001998145 & -0.083962654 & -0.068827082 \\
\hline 12 & 0 & 0.001816685 & -0.085128881 & -0.069984126 \\
\hline 12.1 & 0 & 0.0016366 & -0.086290663 & -0.071136725 \\
\hline 12.2 & 0 & 0.001457885 & -0.08744803 & -0.072284907 \\
\hline
\end{tabular}




\begin{tabular}{|c|c|c|c|c|}
\hline 2.3 & 0 & 0.001280533 & -0.08860101 & -0.073428703 \\
\hline 12.4 & 0 & 0.001104539 & -0.089749634 & -0.074568142 \\
\hline 12.5 & 0 & 0.000929896 & -0.09089393 & -0.075703253 \\
\hline 12.6 & 0 & 0.000756599 & -0.092033926 & -0.076834065 \\
\hline 12.7 & 0 & 0.000584643 & -0.093169652 & -0.077960605 \\
\hline 2.8 & 0 & 0.00041402 & -0.094301134 & -0.079082902 \\
\hline 12.9 & 0 & 0.000244726 & -0.095428401 & -0.080200984 \\
\hline 13 & 0 & 0.0000767543 & -0.09655148 & -0.081314877 \\
\hline 13.1 & 0 & -0.0000899006 & -0.097670399 & -0.082424609 \\
\hline 13.2 & 0 & -0.000255244 & -0.098785183 & -0.083530207 \\
\hline 13.3 & 0 & -0.000419283 & -0.099895859 & -0.084631697 \\
\hline 13.4 & 0 & -0.000582021 & -0.101002454 & -0.085729106 \\
\hline 13.5 & 0 & -0.000743466 & -0.102104993 & -0.086822459 \\
\hline 13.6 & 0 & -0.000903622 & -0.103203503 & -0.087911782 \\
\hline 13.7 & 0 & -0.001062495 & -0.104298008 & -0.088997101 \\
\hline 13.8 & 0 & -0.001220091 & -0.105388535 & -0.09007844 \\
\hline 13.9 & 0 & -0.001376415 & -0.106475106 & -0.091155825 \\
\hline 14 & 0 & -0.001531473 & -0.107557748 & -0.09222928 \\
\hline 14.1 & 0 & -0.00168527 & -0.108636485 & -0.09329883 \\
\hline 14.2 & 0 & -0.001837812 & -0.10971134 & -0.094364499 \\
\hline 14.3 & 0 & -0.001989104 & -0.110782339 & -0.09542631 \\
\hline 14.4 & 0 & -0.002139152 & -0.111849503 & -0.096484287 \\
\hline 14.5 & 0 & -0.002287961 & -0.112912857 & -0.09753845 \\
\hline 14.6 & 0 & -0.002435536 & -0.113972424 & -0.098588834 \\
\hline 14.7 & 0 & -0.002581882 & -0.115028227 & -0.09963545 \\
\hline 14.8 & 0 & -0.002727006 & -0.116080288 & -0.100678324 \\
\hline 14.9 & 0 & -0.002870912 & -0.11712863 & -0.101717479 \\
\hline 15 & 0 & -0.003013605 & -0.118173276 & -0.102752938 \\
\hline 15.1 & 0 & -0.003155091 & -0.119214247 & -0.103784722 \\
\hline 15.2 & 0 & -0.003295375 & -0.120251564 & -0.104812853 \\
\hline 15.3 & 0 & -0.003434462 & -0.121285251 & -0.105837352 \\
\hline 15.4 & 0 & -0.003572357 & -0.122315328 & -0.106858242 \\
\hline
\end{tabular}




\begin{tabular}{|c|c|c|c|c|}
\hline 15.5 & 0 & -0.003709065 & -0.123341816 & -0.107875544 \\
\hline 15.6 & 0 & -0.003844592 & -0.124364737 & -0.108889278 \\
\hline 15.7 & 0 & -0.003978942 & -0.125384111 & -0.109899465 \\
\hline 15.8 & 0 & -0.00411212 & -0.126399958 & -0.110906126 \\
\hline 15.9 & 0 & -0.004244132 & -0.1274123 & -0.111909282 \\
\hline 16 & 0 & -0.004374982 & -0.128421156 & -0.112908952 \\
\hline 16.1 & 0 & -0.004504676 & -0.129426546 & -0.113905156 \\
\hline 16.2 & 0 & -0.004633217 & -0.130428491 & -0.114897915 \\
\hline 16.3 & 0 & -0.004760612 & -0.13142701 & -0.115887248 \\
\hline 16.4 & 0 & -0.004886865 & -0.132422122 & -0.116873174 \\
\hline 16.5 & 0 & -0.00501198 & -0.133413847 & -0.117855713 \\
\hline 16.6 & 0 & -0.005135963 & -0.134402203 & -0.118834884 \\
\hline 16.7 & 0 & -0.005258818 & -0.13538721 & -0.119810706 \\
\hline 16.8 & 0 & -0.005380551 & -0.136368887 & -0.120783198 \\
\hline 16.9 & 0 & -0.005501165 & -0.137347251 & -0.121752378 \\
\hline 17 & 0 & -0.005620665 & -0.138322323 & -0.122718265 \\
\hline 17.1 & 0 & -0.005739057 & -0.139294119 & -0.123680876 \\
\hline 17.2 & 0 & -0.005856345 & -0.140262657 & -0.124640231 \\
\hline 17.3 & 0 & -0.005972532 & -0.141227957 & -0.125596347 \\
\hline 17.4 & 0 & -0.006087625 & -0.142190035 & -0.126549241 \\
\hline 17.5 & 0 & -0.006201628 & -0.143148909 & -0.127498932 \\
\hline 17.6 & 0 & -0.006314544 & -0.144104597 & -0.128445437 \\
\hline 17.7 & 0 & -0.006426379 & -0.145057116 & -0.129388773 \\
\hline 17.8 & 0 & -0.006537137 & -0.146006483 & -0.130328957 \\
\hline 17.9 & 0 & -0.006646822 & -0.146952715 & -0.131266007 \\
\hline 18 & 0 & -0.006755439 & -0.147895829 & -0.132199939 \\
\hline 18.1 & 0 & -0.006862993 & -0.148835841 & -0.133130769 \\
\hline 18.2 & 0 & -0.006969487 & -0.149772769 & -0.134058516 \\
\hline 18.3 & 0 & -0.007074926 & -0.150706629 & -0.134983194 \\
\hline 18.4 & 0 & -0.007179314 & -0.151637436 & -0.135904821 \\
\hline 18.5 & 0 & -0.007282656 & -0.152565207 & -0.136823412 \\
\hline 18.6 & 0 & -0.007384956 & -0.153489959 & -0.137738983 \\
\hline
\end{tabular}




$\begin{array}{lllll}18.7 & 0 & -0.007486218 & -0.154411706 & -0.138651551 \\ 18.8 & 0 & -0.007586446 & -0.155330465 & -0.139561131 \\ 18.9 & 0 & -0.007685645 & -0.156246252 & -0.140467738 \\ 19 & 0 & -0.007783819 & -0.157159081 & -0.141371388 \\ 19.1 & 0 & -0.007880971 & -0.158068968 & -0.142272097 \\ 19.2 & 0 & -0.007977106 & -0.158975928 & -0.14316988 \\ 19.3 & 0 & -0.008072229 & -0.159879977 & -0.144064751 \\ 19.4 & 0 & -0.008166342 & -0.160781129 & -0.144956726 \\ 19.5 & 0 & -0.008259451 & -0.1616794 & -0.14584582 \\ 19.6 & 0 & -0.008351558 & -0.162574803 & -0.146732047 \\ 19.7 & 0 & -0.008442669 & -0.163467354 & -0.147615422 \\ 19.8 & 0 & -0.008532787 & -0.164357066 & -0.148495959 \\ 19.9 & 0 & -0.008621917 & -0.165243955 & -0.149373673 \\ 20 & 0 & -0.008710061 & -0.166128035 & -0.150248578\end{array}$

The Gibbs free energy difference of the four polytypes of boron nitride ( $\mathrm{r}-\mathrm{BN}, \mathrm{h}-\mathrm{BN}, \mathrm{c}-\mathrm{BN}$, and w-BN) as a function of pressure ranging from 0 to $20 \mathrm{GPa}$, calculated with respect to $\mathrm{r}-\mathrm{BN}$ as a reference at a given temperature of 2500 Kelvin. The unit of the Gibbs free energy difference for the four polytypes of boron nitride is given in $\mathrm{eV} /$ atom.

$\begin{array}{lllll}\mathbf{P}(\mathbf{G P a}) \mathbf{r}-\mathbf{B N} & \mathbf{h}-\mathbf{B N} & \mathbf{c}-\mathbf{B N} & \mathbf{w}-\mathbf{B N} \\ 0 & 0 & 0.035716515 & 0.104093232 & 0.117995713 \\ 0.1 & 0 & 0.035386504 & 0.101985889 & 0.115897379 \\ 0.2 & 0 & 0.035053466 & 0.099893076 & 0.113813577 \\ 0.3 & 0 & 0.034717813 & 0.097814562 & 0.111744076 \\ 0.4 & 0 & 0.034379916 & 0.095750121 & 0.10968865 \\ 0.5 & 0 & 0.034040107 & 0.093699532 & 0.107647078 \\ 0.6 & 0 & 0.033698688 & 0.091662583 & 0.105619148 \\ 0.7 & 0 & 0.033355932 & 0.089639067 & 0.103604653 \\ 0.8 & 0 & 0.033012089 & 0.087628781 & 0.10160339 \\ 0.9 & 0 & 0.032667385 & 0.085631531 & 0.099615164 \\ 1 & 0 & 0.032322027 & 0.083647125 & 0.097639785 \\ 1.1 & 0 & 0.031976203 & 0.081675377 & 0.095677066\end{array}$




\begin{tabular}{|c|c|c|c|c|}
\hline 1.2 & 0 & 0.031630088 & 0.079716107 & 0.093726826 \\
\hline 1.3 & 0 & 0.031283838 & 0.077769139 & 0.09178889 \\
\hline 1.4 & 0 & 0.030937601 & 0.0758343 & 0.089863085 \\
\hline 1.5 & 0 & 0.03059151 & 0.073911424 & 0.087949244 \\
\hline 1.6 & 0 & 0.030245689 & 0.072000346 & 0.086047205 \\
\hline 1.7 & 0 & 0.029900252 & 0.070100909 & 0.084156807 \\
\hline 1.8 & 0 & 0.029555302 & 0.068212957 & 0.082277896 \\
\hline 1.9 & 0 & 0.029210938 & 0.066336337 & 0.080410319 \\
\hline 2 & 0 & 0.028867248 & 0.064470903 & 0.078553929 \\
\hline 2.1 & 0 & 0.028524315 & 0.062616509 & 0.076708582 \\
\hline 2.2 & 0 & 0.028182215 & 0.060773014 & 0.074874135 \\
\hline 2.3 & 0 & 0.027841019 & 0.058940281 & 0.073050451 \\
\hline 2.4 & 0 & 0.027500793 & 0.057118174 & 0.071237395 \\
\hline 2.5 & 0 & 0.027161596 & 0.055306561 & 0.069434835 \\
\hline 2.6 & 0 & 0.026823486 & 0.053505313 & 0.067642642 \\
\hline 2.7 & 0 & 0.026486513 & 0.051714305 & 0.06586069 \\
\hline 2.8 & 0 & 0.026150727 & 0.049933414 & 0.064088856 \\
\hline 2.9 & 0 & 0.025816171 & 0.048162517 & 0.062327018 \\
\hline 3 & 0 & 0.025482887 & 0.046401497 & 0.060575059 \\
\hline 3.1 & 0 & 0.025150913 & 0.044650239 & 0.058832862 \\
\hline 3.2 & 0 & 0.024820284 & 0.042908628 & 0.057100316 \\
\hline 3.3 & 0 & 0.024491034 & 0.041176554 & 0.055377307 \\
\hline 3.4 & 0 & 0.024163191 & 0.039453909 & 0.053663728 \\
\hline 3.5 & 0 & 0.023836785 & 0.037740585 & 0.051959473 \\
\hline 3.6 & 0 & 0.023511841 & 0.036036479 & 0.050264436 \\
\hline 3.7 & 0 & 0.023188383 & 0.034341487 & 0.048578515 \\
\hline 3.8 & 0 & 0.022866433 & 0.03265551 & 0.04690161 \\
\hline 3.9 & 0 & 0.022546011 & 0.030978448 & 0.045233622 \\
\hline 4 & 0 & 0.022227135 & 0.029310207 & 0.043574456 \\
\hline 4.1 & 0 & 0.021909823 & 0.02765069 & 0.041924015 \\
\hline 4.2 & 0 & 0.021594091 & 0.025999805 & 0.040282208 \\
\hline 4.3 & 0 & 0.021279952 & 0.02435746 & 0.038648943 \\
\hline
\end{tabular}




\begin{tabular}{|c|c|c|c|c|}
\hline & 0 & 0.02096742 & 0.022723567 & 0.03702413 \\
\hline 4.5 & 0 & 0.020656507 & 0.021098037 & 0.035407682 \\
\hline 4.6 & 0 & 0.020347224 & 0.019480785 & 0.033799512 \\
\hline 4.7 & & 0.020039579 & 0.017871724 & 0.032199536 \\
\hline 4.8 & 0 & 0.019733583 & 0.016270773 & 0.030607671 \\
\hline 4.9 & 0 & 0.019429242 & 0.014677849 & 0.029023834 \\
\hline 5 & 0 & 0.019126565 & 0.013092873 & 0.027447945 \\
\hline 5.1 & 0 & 0.018825557 & 0.011515764 & 0.025879926 \\
\hline 5.2 & 0 & 0.018526224 & 0.009946447 & 0.024319699 \\
\hline 5.3 & 0 & 0.01822857 & 0.008384844 & 0.022767187 \\
\hline 5.4 & 0 & 0.0179326 & 0.00683088 & 0.021222317 \\
\hline 5.5 & 0 & 0.017638317 & 0.005284483 & 0.019685013 \\
\hline 5.6 & 0 & 0.017345724 & 0.003745579 & 0.018155204 \\
\hline 5.7 & 0 & 0.017054823 & 0.002214098 & 0.016632819 \\
\hline 5.8 & 0 & 0.016765616 & 0.000689969 & 0.015117787 \\
\hline 5.9 & 0 & 0.016478105 & -0.000826877 & 0.013610039 \\
\hline 6 & 0 & 0.016192291 & -0.002336508 & 0.012109509 \\
\hline 6.1 & 0 & 0.015908173 & -0.003838989 & 0.010616128 \\
\hline 6.2 & 0 & 0.015625752 & -0.005334387 & 0.009129831 \\
\hline 6.3 & 0 & 0.015345027 & -0.006822766 & 0.007650554 \\
\hline 6.4 & 0 & 0.015065998 & -0.00830419 & 0.006178233 \\
\hline 6.5 & 0 & 0.014788664 & -0.009778722 & 0.004712806 \\
\hline 6.6 & 0 & 0.014513023 & -0.011246423 & 0.003254211 \\
\hline 6.7 & 0 & 0.014239074 & -0.012707353 & 0.001802387 \\
\hline 6.8 & 0 & 0.013966814 & -0.014161574 & 0.000357274 \\
\hline 6.9 & 0 & 0.013696241 & -0.015609142 & -0.001081186 \\
\hline 7 & 0 & 0.013427353 & -0.017050117 & -0.002513052 \\
\hline 7.1 & 0 & 0.013160147 & -0.018484556 & -0.00393838 \\
\hline 7.2 & 0 & 0.012894621 & -0.019912514 & -0.005357227 \\
\hline 7.3 & 0 & 0.01263077 & -0.021334047 & -0.006769648 \\
\hline 7.4 & 0 & 0.012368591 & -0.02274921 & -0.008175698 \\
\hline 7.5 & 0 & 0.012108082 & -0.024158056 & -0.009575431 \\
\hline
\end{tabular}




\begin{tabular}{|c|c|c|c|c|}
\hline 7.6 & 0 & 0.011849238 & -0.025560639 & -0.0109689 \\
\hline 7.7 & 0 & 0.011592055 & -0.026957011 & -0.012356156 \\
\hline 7.8 & 0 & 0.011336529 & -0.028347224 & -0.013737252 \\
\hline 7.9 & 0 & 0.011082656 & -0.029731327 & -0.015112239 \\
\hline 8 & 0 & 0.010830432 & -0.031109372 & -0.016481166 \\
\hline 8.1 & 0 & 0.010579852 & -0.032481408 & -0.017844083 \\
\hline 8.2 & 0 & 0.010330912 & -0.033847483 & -0.019201039 \\
\hline 8.3 & 0 & 0.010083606 & -0.035207646 & -0.020552081 \\
\hline 8.4 & 0 & 0.009837931 & -0.036561943 & -0.021897258 \\
\hline 8.5 & 0 & 0.009593881 & -0.037910422 & -0.023236615 \\
\hline 8.6 & 0 & 0.009351451 & -0.039253129 & -0.024570199 \\
\hline 8.7 & 0 & 0.009110636 & -0.040590109 & -0.025898056 \\
\hline 8.8 & 0 & 0.008871431 & -0.041921407 & -0.027220231 \\
\hline 8.9 & 0 & 0.008633831 & -0.043247068 & -0.028536766 \\
\hline 9 & 0 & 0.00839783 & -0.044567134 & -0.029847708 \\
\hline 9.1 & 0 & 0.008163424 & -0.04588165 & -0.031153098 \\
\hline 9.2 & 0 & 0.007930606 & -0.047190658 & -0.032452979 \\
\hline 9.3 & 0 & 0.007699371 & -0.048494199 & -0.033747393 \\
\hline 9.4 & 0 & 0.007469714 & -0.049792316 & -0.035036382 \\
\hline 9.5 & 0 & 0.007241629 & -0.051085049 & -0.036319987 \\
\hline 9.6 & 0 & 0.007015111 & -0.052372439 & -0.037598248 \\
\hline 9.7 & 0 & 0.006790153 & -0.053654525 & -0.038871204 \\
\hline 9.8 & 0 & 0.006566751 & -0.054931347 & -0.040138896 \\
\hline 9.9 & 0 & 0.006344898 & -0.056202944 & -0.041401363 \\
\hline 10 & 0 & 0.006124589 & -0.057469355 & -0.042658642 \\
\hline 10.1 & 0 & 0.005905817 & -0.058730617 & -0.043910772 \\
\hline 10.2 & 0 & 0.005688578 & -0.059986767 & -0.04515779 \\
\hline 10.3 & 0 & 0.005472866 & -0.061237844 & -0.046399734 \\
\hline 10.4 & 0 & 0.005258674 & -0.062483883 & -0.04763664 \\
\hline 10.5 & 0 & 0.005045996 & -0.06372492 & -0.048868543 \\
\hline 10.6 & 0 & 0.004834828 & -0.064960992 & -0.050095481 \\
\hline 10.7 & 0 & 0.004625163 & -0.066192134 & -0.051317488 \\
\hline
\end{tabular}




\begin{tabular}{|c|c|c|c|c|}
\hline 10.8 & 0 & 0.004416994 & -0.06741838 & -0.052534599 \\
\hline 10.9 & 0 & 0.004210317 & -0.068639765 & -0.053746848 \\
\hline 11 & 0 & 0.004005126 & -0.069856323 & -0.05495427 \\
\hline 11.1 & 0 & 0.003801414 & -0.071068087 & -0.056156898 \\
\hline 11.2 & 0 & 0.003599175 & -0.072275092 & -0.057354765 \\
\hline 1.3 & 0 & 0.003398405 & -0.073477369 & -0.058547905 \\
\hline 11.4 & 0 & 0.003199096 & -0.074674951 & -0.059736349 \\
\hline 11.5 & 0 & 0.003001243 & -0.075867871 & -0.060920131 \\
\hline 11.6 & 0 & 0.002804841 & -0.07705616 & -0.062099282 \\
\hline 11.7 & 0 & 0.002609883 & -0.07823985 & -0.063273832 \\
\hline 11.8 & 0 & 0.002416364 & -0.079418971 & -0.064443814 \\
\hline 11.9 & 0 & 0.002224277 & -0.080593555 & -0.065609258 \\
\hline 12 & 0 & 0.002033616 & -0.081763631 & -0.066770194 \\
\hline 12.1 & 0 & 0.001844377 & -0.08292923 & -0.067926653 \\
\hline 12.2 & 0 & 0.001656554 & -0.084090381 & -0.069078663 \\
\hline 12.3 & 0 & 0.001470139 & -0.085247114 & -0.070226255 \\
\hline 12.4 & 0 & 0.001285128 & -0.086399458 & -0.071369458 \\
\hline 12.5 & 0 & 0.001101515 & -0.087547441 & -0.072508299 \\
\hline 12.6 & 0 & 0.000919294 & -0.088691092 & -0.073642808 \\
\hline 12.7 & 0 & 0.000738459 & -0.089830439 & -0.074773013 \\
\hline 12.8 & 0 & 0.000559006 & -0.09096551 & -0.075898942 \\
\hline 12.9 & 0 & 0.000380927 & -0.092096332 & -0.077020621 \\
\hline 13 & 0 & 0.000204217 & -0.093222932 & -0.078138079 \\
\hline 13.1 & 0 & 0.0000288709 & -0.094345339 & -0.079251342 \\
\hline 13.2 & 0 & -0.000145117 & -0.095463577 & -0.080360437 \\
\hline 13.3 & 0 & -0.000317753 & -0.096577673 & -0.081465391 \\
\hline 13.4 & 0 & -0.000489042 & -0.097687654 & -0.082566228 \\
\hline 13.5 & 0 & -0.000658989 & -0.098793546 & -0.083662976 \\
\hline 13.6 & 0 & -0.000827602 & -0.099895373 & -0.084755659 \\
\hline 13.7 & 0 & -0.000994884 & -0.100993162 & -0.085844304 \\
\hline 13.8 & 0 & -0.001160841 & -0.102086937 & -0.086928934 \\
\hline 13.9 & 0 & -0.00132548 & -0.103176723 & -0.088009576 \\
\hline
\end{tabular}




\begin{tabular}{|c|c|c|c|c|}
\hline 14 & 0 & -0.001488805 & -0.104262544 & -0.089086252 \\
\hline 14.1 & 0 & -0.001650821 & -0.105344425 & -0.090158989 \\
\hline 14.2 & 0 & -0.001811535 & -0.10642239 & -0.091227809 \\
\hline 14.3 & 0 & -0.001970952 & -0.107496463 & -0.092292736 \\
\hline 14.4 & 0 & -0.002129076 & -0.108566666 & -0.093353795 \\
\hline 14.5 & 0 & -0.002285914 & -0.109633025 & -0.094411008 \\
\hline 14.6 & 0 & -0.002441471 & -0.11069556 & -0.095464399 \\
\hline 14.7 & 0 & -0.002595752 & -0.111754297 & -0.09651399 \\
\hline 14.8 & 0 & -0.002748762 & -0.112809256 & -0.097559804 \\
\hline 14.9 & 0 & -0.002900507 & -0.113860461 & -0.098601863 \\
\hline 15 & 0 & -0.003050991 & -0.114907933 & -0.09964019 \\
\hline 15.1 & 0 & -0.003200221 & -0.115951695 & -0.100674807 \\
\hline 15.2 & 0 & -0.003348201 & -0.116991769 & -0.101705735 \\
\hline 15.3 & 0 & -0.003494936 & -0.118028176 & -0.102732996 \\
\hline 15.4 & 0 & -0.003640431 & -0.119060936 & -0.103756612 \\
\hline 15.5 & 0 & -0.003784692 & -0.120090073 & -0.104776603 \\
\hline 15.6 & 0 & -0.003927724 & -0.121115606 & -0.10579299 \\
\hline 15.7 & 0 & -0.004069531 & -0.122137556 & -0.106805795 \\
\hline 15.8 & 0 & -0.00421012 & -0.123155944 & -0.107815037 \\
\hline 15.9 & 0 & -0.004349493 & -0.124170791 & -0.108820738 \\
\hline 16 & 0 & -0.004487658 & -0.125182115 & -0.109822917 \\
\hline 16.1 & 0 & -0.004624618 & -0.126189938 & -0.110821595 \\
\hline 16.2 & 0 & -0.004760379 & -0.127194279 & -0.111816791 \\
\hline 16.3 & 0 & -0.004894946 & -0.128195158 & -0.112808524 \\
\hline 16.4 & 0 & -0.005028322 & -0.129192594 & -0.113796815 \\
\hline 16.5 & 0 & -0.005160514 & -0.130186606 & -0.114781683 \\
\hline 16.6 & 0 & -0.005291526 & -0.131177215 & -0.115763146 \\
\hline 16.7 & 0 & -0.005421363 & -0.132164437 & -0.116741224 \\
\hline 16.8 & 0 & -0.005550029 & -0.133148293 & -0.117715935 \\
\hline 16.9 & 0 & -0.00567753 & -0.134128801 & -0.118687298 \\
\hline 17 & 0 & -0.00580387 & -0.135105979 & -0.119655331 \\
\hline 17.1 & 0 & -0.005929053 & -0.136079845 & -0.120620054 \\
\hline
\end{tabular}




\begin{tabular}{|c|c|c|c|c|}
\hline 17.2 & 0 & -0.006053085 & -0.137050418 & -0.121581483 \\
\hline 17.3 & 0 & -0.00617597 & -0.138017716 & -0.122539636 \\
\hline 17.4 & 0 & -0.006297713 & -0.138981756 & -0.123494532 \\
\hline 17.5 & 0 & -0.006418318 & -0.139942556 & -0.124446188 \\
\hline 17.6 & 0 & -0.00653779 & -0.140900133 & -0.125394622 \\
\hline 17.7 & 0 & -0.006656133 & -0.141854505 & -0.12633985 \\
\hline 17.8 & 0 & -0.006773353 & -0.142805689 & -0.127281891 \\
\hline 17.9 & 0 & -0.006889452 & -0.143753701 & -0.128220761 \\
\hline 18 & 0 & -0.007004437 & -0.144698559 & -0.129156476 \\
\hline 18.1 & 0 & -0.007118311 & -0.14564028 & -0.130089054 \\
\hline 18.2 & 0 & -0.007231078 & -0.14657888 & -0.131018512 \\
\hline 18.3 & 0 & -0.007342744 & -0.147514375 & -0.131944865 \\
\hline 18.4 & 0 & -0.007453313 & -0.148446782 & -0.13286813 \\
\hline 18.5 & 0 & -0.007562788 & -0.149376117 & -0.133788323 \\
\hline 18.6 & 0 & -0.007671174 & -0.150302395 & -0.13470546 \\
\hline 18.7 & 0 & -0.007778476 & -0.151225634 & -0.135619558 \\
\hline 18.8 & 0 & -0.007884698 & -0.152145848 & -0.136530631 \\
\hline 18.9 & 0 & -0.007989844 & -0.153063053 & -0.137438696 \\
\hline 19 & 0 & -0.008093918 & -0.153977265 & -0.138343768 \\
\hline 19.1 & 0 & -0.008196924 & -0.154888499 & -0.139245862 \\
\hline 19.2 & 0 & -0.008298867 & -0.15579677 & -0.140144993 \\
\hline 19.3 & 0 & -0.008399751 & -0.156702094 & -0.141041177 \\
\hline 19.4 & 0 & -0.00849958 & -0.157604485 & -0.141934429 \\
\hline 19.5 & 0 & -0.008598358 & -0.158503958 & -0.142824764 \\
\hline 19.6 & 0 & -0.008696089 & -0.159400529 & -0.143712196 \\
\hline 19.7 & 0 & -0.008792777 & -0.16029421 & -0.144596739 \\
\hline 19.8 & 0 & -0.008888426 & -0.161185018 & -0.14547841 \\
\hline 19.9 & 0 & -0.008983041 & -0.162072967 & -0.146357221 \\
\hline & 0 & -0.009076625 & -0.16295807 & -0.147233187 \\
\hline
\end{tabular}

